\title{
Pre-Revolutionary Russian Photography at the New York Public Library: An Introduction
}

\section{Susan Smith-Peter \& Hee-Gwone Yoo}

To cite this article: Susan Smith-Peter \& Hee-Gwone Yoo (2018) Pre-Revolutionary Russian Photography at the New York Public Library: An Introduction, Slavic \& East European Information Resources, 19:3-4, 263-368, DOI: 10.1080/15228886.2018.1538864

To link to this article: https://doi.org/10.1080/15228886.2018.1538864

曲 Published online: 20 Feb 2019.

Submit your article to this journal $\pi$

Щ Article views: 17

View Crossmark data ¿ 


\title{
Pre-Revolutionary Russian Photography at the New York Public Library: An Introduction
}

\author{
Susan Smith-Peter ${ }^{\mathrm{a}}$ and Hee-Gwone Yoo $^{\mathrm{b}}$ \\ aDepartment of History, College of Staten Island, Staten Island, NY, USA; ${ }^{\circ}$ The New York Public Library, \\ New York, NY, USA
}

\begin{abstract}
This article is an introduction to the New York Public Library's prerevolutionary Russian and Eastern European photographic albums. It also provides a checklist of these albums. The checklist is an especially rich source for Russian architecture, art, and science and provides documentation for a variety of places in the center and provinces of the Russian Empire. Some of the most significant albums are those once owned by the Romanovs and by George Kennan the elder, America's first Russian expert.
\end{abstract}

\section{KEYWORDS}

The New York Public Library; Russian and Soviet photography; checklists; Pre-revolutionary Russia; Romanovs; Russian Empire; Russification; style russe

The New York Public Library has an exceptional collection of pre-revolutionary Russian photography. Much of this pre-revolutionary material originally belonged to the Russian imperial family. The albums provide a vivid portrait of the cultural flowering of the late Imperial period, from art and architecture to depictions of nationalities and provinces. They illustrate the history of photography in Russia and include works from many of the most significant pre-revolutionary Russian photographers. The materials also speak to Russian-American relations through albums belonging to the first American expert on Russia, George Kennan, 1845-1924. As many of these materials are not included in the library's main (classic) catalog, scholars might easily overlook them, which is why this checklist is therefore especially important.

From 1899 to 2008, The New York Public Library had a division dedicated to Slavic materials. Due to petitioning from Russian speakers in 1899, the Russian Division was created. Later, the name was changed to the Slavonic, and then in the late 1980s, to the Slavic and Baltic Division. The division saw much growth under the leadership of Avrahm Yarmolinsky from 1918 to 1955. During the 1920s, when the Soviet government sold many treasures for hard currency, the division acquired many priceless items, including some of the Romanov photograph albums listed in the checklist. While there were times of less activity due to the financial problems of the 1970s, the last curator of the division from 1984-2009,

CONTACT Susan Smith-Peter Susan.smithpeter@csi.cuny.edu $\Theta$ Department of History, College of Staten Island/CUNY, 2800 Victory Blvd., 2N-215, Staten Island, NY 10314, USA

All photos taken by Hee-Gwone Yoo of images in the New York Public Library's collection.

Susan Smith-Peter is sole author of this introduction and is co-author, with Hee-Gwone Yoo, of the checklist. 
Edward Kasinec, oversaw an active program of buying and soliciting new works, describing the division's riches, and staging major exhibitions. ${ }^{1}$

In 2008, as part of a controversial plan to reshape the library, the Slavic and Baltic Division was closed and many of its employees were moved to other positions where they did not have contact with the public. During the aftermath of the closure, access to Slavic, East European, and Eurasian items became very difficult. After a 2008 National Endowment for the Humanities Institute at the NYPL, I organized the Committee in Support of Slavic and Baltic Scholarship, which advocated for the needs of the Slavic materials and access to them. As a result, the library placed Hee-Gwone Yoo, a former member of the division, in a public service position. In addition, Steven Corrsin, who is the full-time curator at the library's Dorot Jewish Division, was also given responsibilities as a Slavic curator. He oversaw the transfer of the photographic albums to the Miriam and Ira D. Wallach Division of Art, Prints and Photographs. The checklist itself is one of the last projects to emerge from the Division. In October 2018, Bogdan Horbal was named the new full-time Slavic curator at the library.

The checklist was part of a larger project to make Slavic visual resources more accessible by publishing descriptions of important collections. ${ }^{2}$ The albums are now located in the Prints and Photographs Study Room, which is equipped with expert staff who know how properly to store the items and who allow access to them using cradles as needed. Many of the materials are oversized or fragile. As textual materials associated with the photographs, such as introductions or any text-only pages, were not digitized, physical access to the works remains important even if they have been digitized as the textual material provides important context for the visuals. The existence of additional textual material is generally not noted in the digitized versions, so the checklist remains an invaluable resource about the collections. Not all the material has been digitized, particularly items that were acquired in the division's final years. Several important post-revolutionary collections have recently been digitized as part of this process. In the course of working through the items, several were found in need of restoration and the division is addressing these issues.

There are problems with access to the material in terms of finding aids. Most of the albums, particularly the pre-revolutionary ones, are digitized, but generally without information on the physical location of the materials, aside from references to the now-closed Slavic and Baltic Division. Most of the albums are not listed in the library's main online catalog so most scholars would not come across them if they searched, even for the exact topic of the album. Many albums are listed in the Prints and Photographs Online Catalog, which is separate from the main catalog and requires a separate search on the NYPL website. Accordingly, most scholars, apart from art historians, would not know where to search for them. Photography 
catalogers also do not have the access to add entries to the main catalog, which means that additions to the Prints and Photographs Catalog do not appear there. In addition, the cataloging of the albums is photograph by photograph, which means that those photographs without captions are difficult to catalog at all. Also, the Prints and Photographs Catalog is inconsistent in its use of diacritics, which means that titles (i.e. Al'bum) should be searched both with and without diacritics. As a result, the SLV number, which is the current call number, is given in the checklist to assist with accessing the material. Historical call numbers (usually starting with Slav Reserve) are also retained as a backup. The checklist only includes photographic material bound into albums. There are Slavic collections that include loose photographs that are not in the checklist. Some of these are stored offsite and can be used by patrons without significant monitoring by library staff.

I revised the Yoo checklist using a de visu examination of all the items with assistance from Galina Mardilovich. In so doing, I gained a deeper understanding of the importance of working with originals. In instances where captions were written on the back of photographs, they have been included, but not digitized. In other cases, the photographs are tipped in to books whose content cannot be fully described even in the checklist. The materials in the pre-revolutionary checklist are especially significant in the following areas: the history of the Romanov family; the history of photography; the history of art and architecture; history of the provinces; and RussianAmerican relations.

The albums related to the Romanov family were presented to members of the family or family photograph albums belonging to the imperial family. For example, two splendid oversize albums on the Turkestan Exhibition of 1886 (SLV 124) and of 1890 (SLV 125) were presented to Emperor Alexander III. A similarly impressive album belonging to Alexander III contains photographs of Suzdal' monasteries (SLV 118). A considerable number of albums belonged to Grand Duke Vladimir Aleksandrovich, 1847-1909, who was for many years the president of the Imperial Academy of Fine Arts and a patron of the Ballets Russe. One album documents his travels abroad in 1870 (SLV 093). Another album, presented to Grand Duchess Anastasia Nikolaevna, 1901-1918, provides documentary material on a World War One era medical train (SLV 035). An uncatalogued bourgeois-style small photograph album contains carte de visite portraits of Romanov family members from the 1860 s and 1870s, beginning with Alexander II (SLV 101).

Other albums provide examples of the works of important Russian photographers. An album of portraits by Andrei Denier, 1820-1892, is 
an example of the prominence of portraiture in Russian photography in the 1860s (SLV 004). There are two albums by Andrei Karelin, 1837-1906, one of Russia's leading nineteenth century photographers (SLV 058 and 059) and an album of ethnographic types by Jean Raoult (SLV 099).

The genesis of the style russe can be traced through several of the albums as well as a broader history of the art and architecture of late Imperial Russia. As mentioned earlier, there are several albums belonging to Grand Duke Vladimir Aleksandrovich, whose leadership of the Imperial Academy of Arts was instrumental in developing an internationally recognized Russian style. We can see the process of gathering visual evidence of pre-Petrine Russian architecture in the Grand Duke's albums of the sights of Sol'vychegodsk (SLV 131), Tot'ma (SLV 132), and Velikii Ustiug (SLV 133). This new Russian style was presented at world's fairs like the World's Columbian Exposition in Chicago in 1893. The library has an album (SLV 154) with views of the Russian section of the exposition presented to the Grand Duke. His bookplate is also present in an album of photographs of professors and students of the Academy of Arts (SLV 005). More broadly, the history of architecture, especially church architecture, is well represented, with albums, some quite lavish, of the Church of Christ the Savior in Moscow (SLV 063 and 064), churches in Iaroslavl' (SLV 011 and 021), monasteries in the Crimea (SLV 006), and others.

Albums related to the history of the provinces are especially rich in the documentation of exhibitions there, including the All-Russian exhibition in Nizhnii Novgorod in 1896, with a bookplate of Grand Duke Konstantin Konstantinovich, 1858-1915 (SLV 148). Other exhibition albums are from those held at Borovichi (SLV 002) and Kostroma (SLV 008).

Materials related to Russian-American relations include an album of Russian settlers in the US in the 1910s, which seems to have resulted from a cross-country trip sponsored by Russian ambassador to the US, Boris Bakhmeteff, 1880-1951 (SLV 045). The bulk of the materials in this category, however, once belonged to the first American expert on Russia, George Kennan. These include photographs of the Caucasus (SLV 061) and a particularly interesting album of Russian political exiles and convicts (SLV 062) related to his major work, Siberia and the Exile System (1891). An album of photographs of the penal colony of Sakhalin (SLV 110) has captions written in a hand very similar to Kennan's. There are two uncatalogued Kennan albums: the first deals specifically with the natural scenery of Siberia (MFYH 01-9040) while the second contains a broad range of photographs of Siberia (MFY+ 03-4889). As it was Kennan's work that led 
to a shift in American views of Russia from generally positive to negative, this material is especially important.

Some fascinating items do not fit into these categories, such as a photograph of the poet Nikolai Gumilev with a signature and stamp from a Tsarskoe Selo policeman, dated July 3, 1906, testifying that it is indeed his image (SLV 042).

In conclusion, the importance of the material is quite significant. This checklist should serve to make it more accessible to scholars. Collaborative work might result in interesting findings and raise the profile of the materials. For example, I worked with my MA students on an exhibit to commemorate the one hundredth anniversary of the Russian Revolution. We used a photographic album from Bessie Beatty, an American journalist who wrote about her experience of the Revolution, along with other materials such as the John Reed collection of posters and proclamations, also held by the library, for an exhibit entitled "The Russian Revolution: American Perspectives." Hopefully, this checklist will encourage other scholars to use the materials as well.

\section{Disclosure statement}

No potential conflict of interest was reported by the author.

\section{Notes}

1. Robert H. Davis, Jr., Slavic and Baltic Library Resources at the New York Public Library: A First History and Practical Guide (New York: The New York Public Library, 1994); Cynthia Whittaker, ed., Russia Engages the World, 1453-1825 (Cambridge, MA: Harvard University Press, 2003).

2. Hee-Gwone Yoo and Kristen Regina, Visual Resources from Russia and Eastern Europe in the New York Public Library: A Checklist (New York: Ross Publishing, 2008); Slavic \& East European Information Resources 11, no. 2-3 (2010).

\section{Checklist}

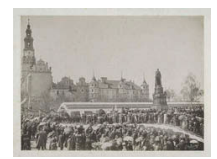

Akt sdachi pamiatnika, sooruzhennago na sredstva sel'skago naseleniia gubernii Tsarstva Pol'skago Imperatoru Aleksandru II-u v gorodie Chenstokhovie.

[Częstochowa], 1890. [1] p., 8 mounted original photographs, $40 \times 55 \mathrm{~cm}$. Album marking the dedication of the monument in honor of Alexander II in Częstochowa, Poland on April 17, 1899. Contains one page of the document signed by the architect I. V. Maevskii and other officials; contemporary binding, gold stamped. Purchased from Alex Rabinovich. Maevskii, Ilarion Vikentevich. Alexander II, Emperor of Russia, 1818-1881. Monuments - Poland - Częstochowa. Slav. Reserve (Photo) 96-4456. SLV 
001 [Cover title]; Fasad ploshchadki i liestnitsy pod pamiatnik Imperatoru Aleksandru II v gorodie Chenstokhovie; Plan ploshchadki i liestnitsy pod pamiatnik Imperatoru Aleksandru II v gorodie Chenstokhovie; Prodol'nyi razriez ploshchadki i liestnitsy pod pamiatnik Imperatoru Aleksandru II v gorodie Chenstokhovie; Proekt postroiki storozhki pri pamiatnik v g. Chenstokhovie; [Inauguration of monument to Alexander II]; [Monument to Alexander II in Czestochowa]; [Monument to Alexander II]; [Inauguration of monument to Alexander II]

\section{Al'bom Borovichskoi oblastnoi sel'sko-khoziaistvennoi, kustarnoi i promyshlennoi vystavki, 1894.}

A. A. Agrafioti, n.d. 34 mounted photographs on 17 leaves, $28 \times 34 \mathrm{~cm}$. Binder's title. With descriptive letterpress. At head of title: Ego Imperatorskoe Vysochestvo Velikii Kniaz' Vladimir Aleksandrovich. Binding of grained brown morocco, gilt. Cream moire silk doublure.

Title page: [His Imperial Highness the Grand Duke Vladimir Aleksandrovich- Coat of Arms-Album of the Exhibition of Agriculture, Handicraft and Industry at Borovichi]. "Album was documented well by subjects, especially regarding the architecture of the fair: museum, theater, pavilions (showing the pavilion of the photographer A. A. Agrafioti). The inside views of founding of the firms, description of production, number of workers employed, annual income, number of machines, etc. The products range from beer and alcohol, to Portland Cement, roofing paper, and book binding." (Pierre Apraxine) Borovichskii uiezd (Russia). Exhibitions Russia - Borovichskii uiezd.

Slav. Reserve Photo (Borovichi) SLV 002 [Cover title]; Vid goroda Borovichi; Priezd Ego Vysochestva Printsa A. P. Ol'denburgskago; Muzei; V. A. Bel'ter. Predsied M. Ia. Shul'gin; A. N. Vul'f. Tov. Predsied. M. V. Shadrin. Sekretar' E. Ia. Auslender; S. A. Vislenev. I. V. Malinin; Muzei (Vnutr); Glavnyi vkhod Vystavki; Promyshlennyi otdiel; Teatr i restoran; Sel'sko-khoziaistvennyi otdiel; Zavodsko-promyshlennyi i mashinnyi otdiel; Obshchii vid Vystavki; Pitomnik liesnago Departamenta; Liesnoi Zemskii i kustarnyi otdiel; Fotograficheskii pavil'on A. A. Agrafioti: Upolnomochennyi na Borovichskoi Vystavkie slieduiushikh firm; Firma Vladimir Vishniakov...; Obshchestvo Bekman i Ko...; Firma A. Nauman i Ko...; Pravlenie SPB...; Firma “Astra” sushch. s 1889 g...; Firma "Slon” osnov. v 1894 g...; Firma Urlaub osnovana v 1870 g....; G. Zh. Perro-Minno...; Firma S.-Peterburgskoi kosmetichekoi laboratorii A. Englund...; Firma A.M. Zhukov osnovana v 1845 g...; Aktsionernoe Obshchestvo "Bavariia" osnov. v 1863 g.; Aktsionernoe Obshchestvo Pivovarennyi zavod "Novaia Bavariia" osnov. v 1876 g.; Firma Khardin i Miller osnov. v 1885 g...; Torgovyi dom Liman i Riks v S.-Peterburgie; sushch. s 1874 g...; Firma V.A. Parman, osnovana 
v 1877 g...; Deshevyi podiezdnoi put'...; Firma E.I. Gleitsman sushchesv. s 1871 g...; Perepletnaia masterskaia A. Shnel', osnov. v 1886 g...; Firma Matviei Iakovlevich Shul'gin, sushchest. s 1786 g...; Iakov Loginovich Loginov...

[Al'bom Dukhovnoi Missii]. [S.l.: s.n., 1881?]. 48 mounted photographs, $32 \times 45 \mathrm{~cm}$.

Cover-title. No text; photographs have no captions. Photographs taken in 1881 during the visit of Grand Duke Sergei Aleksandrovich (1857-1905) to the Mission in Jerusalem.

Binding of Red plush with olive wood panels, with inlaid mosaic border, on sides. On front panel is mounted wood-carving of the Mission with legend reading: "Al'bom Dukhovnoi Missii." Slavic Recon Project. Metropolitan New York Library Council, 2008. Russkaia pravoslavnaia tserkov. Russkaia dukhovnaia missiia v Ierusalime.

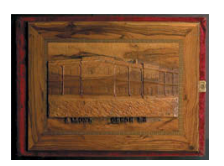

Slav. Reserve (Photo) (Albom dukhovnoi missii) SLV 003 [Cover title]; [Landscape of a wall around a cathedral]; [Cathedral in Jerusalem]; [Cathedral]; [Interior view of the Church of the Holy Ascension at the Mount of Olives Convent of the Russian Orthodox]; [View of a church yard and building]; [Interior view of a church (main altar)]; [The Russian Orthodox Church of St. Mary Magdalen at Gethsemane on the base of the Mount of Olives]; [The Russian Orthodox Church of St. Magdalen and its outside view]; [The Russian Orthodox Church of St. Magdalen on the Mount of Olives]; [The Russian Orthodox Church of St. Magdalen on the Mount of Olives]; [A view of the valley behind the Church of St. Mary Magdalen]; [View of Old City of Jerusalem and the Church of St. Mary Magdalen]; [An altar and icons]; [Fresco of an angel]; [Church and its bell tower]; [Church and its bell tower]; [Church and its bell tower]; [Interior view of the church (main altar)]; [View of cupola with Christ and 24 elders]; [A building beside the church]; [Mosaics]; [Landscape (a church in the horizon with the bell tower)]; [Remnants of an ancient building]; [A grotto underground of an ancient house]; [General view of the outskirts of Jerusalem]; [Views of a church, bell tower, and remains of ancient buildings in the foreground]; [Unidentified Church]; [Interior view of a church with a group of nuns]; [General view of a church surrounded by a wall]; [Unidentified church]; [Façade of the church and a clergyman]; [Church in the valley surrounded by trees - 2 photos]; [Outdoor service by Russian clerics near a tree]; [Ecclesiastical building]; [Ecclesiastical building]; [A bell tower in the middle of ancient walls]; [Woman and building]; [Unidentified building]; [Unidentified building]; [A garden near the building - 2 photos]; [Modern house and a clergyman]; [Modern house]; [Building and its construction]; [The construction of the building]; [Ancient ruins of houses in the 
waterfront]; [Grand Duke Sergei Aleksandrovich with an Archimandrite]; [Grand Duke Aleksandrovich and the Archimandrite with pilgrims]

\section{Al'bom fotograficheskikh portretov avgustieishikh osob i lits, izviestnykh} $v$ Rossii.

St. Petersburg: G. Dener (Denier), 1865. 128 mounted photographs: ill., ports.; original book $10 \times 22 \mathrm{~cm}$. Albumen carte de visite-size prints, mounted, each with a caption. Apparently issued in monthly fascicules. The only cover preserved is dated February, 1865. Title page illustration by Adolf Charlemagne. Bookplate of Grand Duke Vladimir Aleksandrovich (18471909), with his initials on cover. "Perlstein Jun 18, 1931" on the first interleaf appears the red stamp. "The majority of portraits are of men in the government, army, or at court. There are 12 ports. of members of Imperial family, and 6 of figures of the Church. There are portraits of Academicians and professors, writers, and theatre people: 7 portraits of ballet dancers (including M. S. Petipa). There are portraits of Russian types, alone or in groups (Georgians, Mongolians, etc.). The portraits follow each other at random. Some sitters are indicated as deceased. Some portraits (M. S. Petipa, A. M. Karatygina [actress], Grand Duchess Maria Alexandrovna, Count P.P. Shuvalov) are excellent. The prints are mostly very good and the overall quality of the album is very high. Besides being an excellent example of its type, the album is quite unusual in its mix of official portraiture, ethnography, and dancers in tutus." (Pierre Apraxine) Master Negative ${ }^{\star} Z Z-30653 \&{ }^{*}$ ZQ-1020 Celebrities - Russia. Persons-Russia.

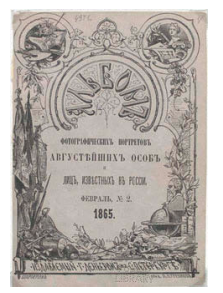

Slav. Reserve (Photo) (Albom fotograficheskikh) SLV 004 [Cover title]; E.V. Velikii Kniaz' Pavel Aleksandrovich; A.E. Timashev; E.I. V. Velikii Kniaz' Vladimir Aleksandrovich; N.A. Nekrasov; E.I.V. Velikaia Kniazhna Mariia Aleksandrovna; Aleksandr II Imperator Vserossiiskii; Gosudar' Nasliednik Tsesarevich Nikolai Aleksandrovich; K.M. Ber. Akademik; F.P. Litke. General Adiut. Admiral Prezid. Imper. Akad. Nauk; Kniaz P.P. Gagarin; Kniaz P. A. Viazemskii; F.I. Iordan, professor gravirovaniia; M.S. Kutorga, Akademik Dieistv. St. Sov.; L.I. Gergard, General-maior, direktor Telegrafov; N.A. Gerngros, Taik. Sov. Tov. Ministra Gosud. Imush.; A.N. Sutgob, GeneralLeitenant; Svietl. Kniaz E.L. Vitgenshtein, Svity Ego Velich. General-Maior; Graf. S.S. Lanskoi, byvshii Ministr Vnutr. Diel. (skonchavsh); Svietl. Kniaz V. D. Golitsyn. Nachal'n 1-I Gvard. Kaval. Divizii; E.I.V. Velikii Kn. Nikolai Nikolaevich; P.L. Chebyshev, Chl. Uchen. Komiteta, Dieistv. St. Sov.; A.A. Kraevskii, Redaktor gazety "Golos"; V.V. Pelikan, Sovieshch. Chl. Med. Sovieta, Tainyi Sovietn.; A.G. Troinitskii, Tainyi Sovietn., Tovarishch Min. Vn. Diel.; K.K. Grot, Stats-Sekretar', Tainyi Sovietn.; K.N. Pos'et, Svity Ego Velich. Kontr-Admiral; Ego Imp. Vysoch. Velikii Kniaz' Konstantin Konstantinovich; Graf N. T. Baranov 1-i, Gener.-Adiutant, gener.-Leitenant; M. Kh. Reitern, 
Ministr finansov; Gertsog Osuna-i-Infantado, Ispanskii posol pri Dvorie Ego Velichestva; A.A. Barantsov 1-i, Tovarishch General-Fel'dtseikhmeistera, Gener.Adiut., Gener.-Leitenant; Grafinia M.G. Razumovskaia, Kavalerstv. Dama (skonchavshaiasia); E.V. Velikaia Kniaginia Ol'ga Feodorovna; E.V. Velikii kniaz' Mikhail Nikolaevich; V.G. Benediktov, Poet; E.V. Rodzianko, Nachal'n. Uch. Ord. Sv. Ekateriny, Kavalerstven Dama; E. Imp. Vys. Vel. Kn. Konstantin Nikolaevich; I.K. Aivazovskii, Professor morskoi zhivopisi; G.G. Vilamov, Gener.-Leitenant; A.P. Bezak, Kievskii Voennyi Gener.-Gubernat; P.A. Valuev, Ministr Vnutr. Diel; N.I. Grech, Russkii Pisatel', rod. 1787; Kniaz' A.I. Bariatinskii, Komand. L.-Gv. Preobrazh. Polka; Graf A.V. Adlerberg 2-i, Koman. Glavn. Kvart., Gener.-Adiutant, Gener.-Leiten; Gosudar' Nasliednik Tsesarevich, Aleksandr Aleksandrovich; Graf F.F. Berg. Gener. Adiut. Gener. ot infanterii, Namiestnik v Tsarstvie Pol'skom; Kniaz' V.A. Dolgorukov 1, Shef korpusa zhandarm., General ot kavalerii; I.I. Sosnitskii, Artist Imper. Teatrov (v roli Gorodnichago v Revizorie); Iu. N. Linskaia, Artistka Imper. Teatrov. (v roli Tsarskoi mamy, iz dr. Boiarin Matvieev); Graf N.N. Evdokimov; Svietl. Kn. Italiiskii, Graf. A.A. Suvorov-Rymnikskii; A.I. Butakov 2-i, Sv. E.Velich. KontrAdmiral; Baron A.K. von Tetenborn, Svity E. Velichestva Gener-Maior; Baron E. F. Meiendorf, Gener.-Adiut. Gener.ot Kavalerii; S.A. Khrulev, General-Leitenant; N.O. Sukhozanet, Gener.-Adiut., Gener. ot Artillerii; A.I. Panfilov, VitseAdmiral; P.K. Meiendorf, Ober-Gofmeister Dvora Ego Velichestva (skonchavshiisia); A.P. Akhmatov, General-Adiutant, General-Leitenant; M.G. Khomutov, General ot Kavalerii, byvshii Nakazn. Ataman Voiska Donskoi (skonchavshiisia); Ego Imp. Vysoch. Velikii Kniaz' Nikolai Konstantinovich; A.I. Filosofov, GenerAdiut., General ot Artillerii; Kniaz' D.O. Bebutov, General-Leitenant; E.V. Brimmer, General-leitenant; Kniaz' D.A. Obolenskii, Stat-Sekretar, Tainyi Sovietnik; Graf P.P. Shuvalov, Kamerger Dvora Ego Velichestva; G.Kh. Gasfort, Gener. ot Infant., Chlen Gosud. Sovieta; F.S. Paniutin, Gener.-Adiut., Chlen Gosud. Sovieta (skonchavshiisia); S.D. Bezobrazov 1-i, Gener.-Adiut., General ot Kavalerii; F.S. Tsytsurin, Pochetn. Leib-Medik, Direktor Voen.Medits. Departamenta; Graf F.V. Orlov-Denisov, gener.-Adiut (skonchavshiisia); F.I. Prianishnikov, Dieistv. Tain. Sov., Chlen Gosud. Sov.; V.B. Bazhanov, Dukhovnik Ikh Imp. Velich. i Vysoch.; A.N. Nikitenko, Ord. Akademik Imp. Akad. Nauk; V.F. Fon der Launits. General-adiutant (skonchavshiisia); P.A. Kolzakov, gener.- adiut., admiral (skonchavshiisia); N.A. Miliutin, StatsSekretar', Tain. Sov.; A.M. Kniazhevich, Dieistv. Tain. Sov. Chlen Gosud. Sov.; A.F. Minkvits, Nachal'nik Shtaba Varshav. Voen. okruga, Gener.-Adiut.; P. Kh. Grabbe 1., Nakaznyi Ataman Voiska Donskago; Graf A.N. Liders, Gener.-Adiut., Gener. ot Infant.; D.A. Miliutin, Gener. Adiut. Gener.-Leiten., Voennyi Ministr; Kniaz' A.M. Gorchakov, Vitse-Kantsler, Ministr Inostrannykh Diel; Graf V.F. Adlerberg, Ministr Imper. Dvora; F.I. Tiutchev, Kamerger Dvora E. Imp. Velichestva; A.F. Diugamel, Komand. Voisk. V Zapadn. Sibiri, Gener. ot Inf.; Arkhimandrit Vladimir, Inspektor S.-Peterb. Dukhovnoi Akademii; Sofoniia, 
Episkov Novomirgorodskii; N.V. Kukol'nik, Pisatel'; P.A. Morits, Sekretar Eia Velichestva; K.A. Ton, Rektor Imp. Akad. Khudozh. po chasti Arkhitektury; D.I. Grimm, Professor Arkhitektury; V.F. Korshch, Redaktor i Izdatel' S.-Peterb. Viedomostei; Taska, Artist Italianskoi opery Imperat. Teatr; N. V. Gerbel, izdatel' sochinenii Shillera v perevodie na Russkii iazyk; V.E. Ekk, Professor Imp. Mediko-Khirurgich. Akad.; P.G. Riedkin, byvshii profes. Moskovsk. Universiteta, Tainyi Sovietnik; N.F. Edekauer, Leib-Medik, Professor; V. S. Kurochkin, Izdatel'"Iskry"; A. E. Martynov, Artist imp. Teatr. (skonchavshiisia); F. A. Snietkova, Artistka Imp. Teatr (Ostaviv. stsenu); Deveriia, Artistka Imp. Teatr. frantsuzskoi stseny; F. A. Bruni, Rektor Imp. Akad. Khudozhestv; M. S. Petipa, Baletn. Artistka Imp. Teatrov; G-zha M. N. Madaeva, Baletnaia artistka Imp. Teatr.; Lomonosov, Russkii Pisatel'; V. A. Karatygin, Artist Impera. Teatr. (skonchavshiisia); A. M. Karatygina, byvshaia Artistka Imp. Teatr; G-zha Kemmerer, Baletnaia artistka Imp. Teatr; A.D. Kosheva, Baletn. Artistka Imp. Teatr; V. I. Lapshina, Baletn. Artistka Imp. Teatr; E. V. Velikaia Knizhna Ol'ga Konstantinovna; N. N. Amosova 2-ia, Baletn. Artistka Imper. Teatrov; Stefanskaia, baletnaia artistka Varshavskago teatra (fotografiia G-na Faiansa v Varshavie); O.P. Radina 3-ia, Baletn. Artistka Imp. Teatrov; Tipy Manchku. Iz primorskago kraia Amurskoi oblasti; Tipy: Malorossiianin; Tipy: "Gruzinki"; Tipy: Mingrel'tsy; Tipy: "Samoiedy”, Arkhagelskoi gubernii; Dzhigety. Na vostochnom beregu chernago moria fotogr; Glav. Sht. Kav. Armii; Tipy: Mushi; Tipy: Khevsury; Mitropolit Kievskii i Galitskii Arsenii; Isidor, Mitropolit Novgorodskii, S.-Peterburgskii, Estliandskii i Finlandskii; Ioann, episkop Poltavskoi i Pereiaslavskii; I. F. Gorbunov, Artist Imp. Teatr (stsena iz Narodnago byta)

\section{[Al'bom fotografii prepodavatelei $i$ uchenikov Imperatorskoi akademii khudozhestv.]}

St. Petersburg: Académie impériale des beaux arts, 1893. 7 mounted photographs, $29 \times 37 \mathrm{~cm}$.

"An album of 7 albumen prints encased in gray mounts embossed with decoration, and with printed caption: Academie Imperiale des Beaux Arts St Petersbourg, 1893. Photographie Internationale de Jongh Freres. Rue de Longchamps, 15 Nevilly - Paris (in French). The album was bound by the N. Y.P.L. A red stamp on the inside of back cover: Mar 8 - 1934.

Group portraits of students and teachers at the Imperial Academy of Fine Arts. End of the year type of group portraits: the students (pl. 1-4) sitting and standing up to 6 rows. The professors (pl. 5-7) in smaller groups and closer, up to 3 rows. Plate 1 and 2 show only men among students. Plate 3 and 4 show some women ( 7 out of a total of 55 , and 7 out of 62 ). There are no women among the teachers. The photographs have no aesthetic interest, only an iconographic one, or as a document on the Academy. Mounts and prints in good condition, but 
surface abrasions on pl. 4. The mounts are getting loose from the binding." (Pierre Apraxine) Imperatorskaia akademiia khudozhestv (Russia). Saint Petersburg (Russia).

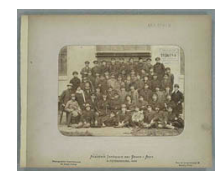

Slav. Reserve (Photo) (Imp. aka. khud. Albom fotografii) SLV 005 Academie Imperiale des Beaux-Arts....

\section{Al'bom Georgievskago Balaklavskago monastyria v Krymu, Chast' 1}

[Russia?, 1900?] 8 photographs, $20 \times 26 \mathrm{~cm}$.

Cover title. An album of eight black and white mounted photographs. "Chast' 1"-Cover. Formerly in the collection of Alexandra, Empress (18721918), consort of Nicholas II (r. 1894-1918). Library's copy lacks the eighth photograph. Bookplate of Alexandra, Empress, consort of Nicholas II on inside back cover. Imperial presentation copy bound in purple velvet with gold letters and engravings. Georgievskii monastyr (Crimea, Ukraine).

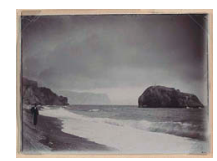

Slav. Reserve (Photo) 93-4844. SLV 006 [Cover title]; [General view of island and part of monastery]; [Cliff beside Balaklavskii Georgievskii monastery]; [Cliff of Balaklavskii Georgievskii monastery]; [General view of Balaklavskii Georgievskii monastey]; [Cliff of Balaklavskii Georgievskii monastery]; [Cliffs on the Island]; [Cliff of Balaklavskii Georgievskii monastery]

Al'bom izdannyi ko dniu 75-letniago iubileia L. Gv. 1 Strielkovago Ego Velichestva polka: [1856-1931].

[Russia?: s.n. 1931]. 33 photographs (chiefly mounted), $18 \times 28 \mathrm{~cm}$. "Kratkaia pamiatka Leib-Gvardii 1-go Strielkovago Ego Velichestva polka." Memorial album of original photographs of First, His Majesty's Own Rifles. Accompanied by: Kratkaia pamiatka Leib-Gvardii 1-go Strielkovago Ego Velichestva polka. The Oleg Olegovich Pantuhoff Collection, donated to the NYPL in his memory by Oleg Olegovich Pantuhoff (Col. John L. Bates).

DOE Title II-c Project "Old and Rare." Russia. Armiia. Leib-Gvardii Strelkovyi polk, I.

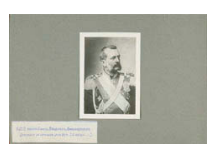

Slav. Reserve 90-4456 no. 79 (Bates) SLV 007 Znamia L. Gv. 1 Strielkovago Ego Velichestva bataliona; Ikona Sv. Zosimy i Savvatii, blagoslovenie Solovetskago monastyriia...; Ego Imperatorskoe Velichestvo Derzhavnyi Osnoviatel' i Shef batal. Gosudar' Imperator Aleksandr II Nikolaevich; Derzhavnyi Shef batal. E.I.V. Gosudar' Imperator Aleksandr III Aleksandrovich; Ego Imperatorskoe Velichestvo Gosudar' Imperator Nikolai II Aleksandrovich v iiune 1907; Ego Imp. Vys. Avgusteishii strelok Nikolai Aleksandrovich; Avgusteishii strelok E.I.V. Naslednik Tsessarevich i velikii kniaz' Aleksei Nikolaevich v stroiu Gosudarevoi roty; E.I.V. Velikii kniaz' Vladimir Aleksandrovich; Ego Velichestvo Gertsog Meklenburg-Strelitskii, Inspektor strelkovykh 
batalionov...; Ego Vysochestvo Gertsog Georgii Meklenburg-Strelitskii...; Komandir L.Gv. 1 Strelkovago Ego Velichestva polka Sv.; E.V. gen. Maior P.T. Nikolaev, vystupivshii s polkom na voinu v 1914 godu; Komandir L.Gv. 1 Strelkovago Ego Velichestva polka Sv. E.V. g. m. Levstrem, priniavshii polk 17 sent. 1914 g. i komandovavshii im do sered. Fevralia 1917 g.; Leib-Gvardii 1-ii Strelnovyi batalion na ploshchadi Zimniago dvortsa v S-Peterburge v 1857 godu; [Aleksei Nikolaevich among officers in 1914/Nicholas II and Aleksei in Tsarskoe Selo in 1914]; Znamenshchik Fel'dfebel' Kapustin, na ploshchadke Bol'shago Tsarskosel'skago dvortsa; Znamia L. Gv. 1 Strielkovago Ego Velichestva polka na pokhod v 1915 g. znamenshchik st. unt. of. Sofronov; Parad na ploshchadi bol'shago Tsarskosel'skago dvortsa v den' batalionnago prazdnika v 1905 g.; Gruppa ofitserov v den' parada batalionnago prazdnika v 1905 godu; Znamia L. Gv. 1 Strielkovago Ego Velichestva polka s chastiu znamennago otdeleniia...1914; Komandir polka gen.maior Levstrem i Arkhimandrt Ambrosii; Zavtrak ofitserov L. Gv. 1 Strielkovago Ego Velichestva polka. Noiabr 1914 g.; Nach. Divizii gen. Leit. Del'sal' s ofitserami L. Gv. 1 Strielkovago Ego Velichestva polka...; Klinok shashki s polkovym shit'em: Zavedeno $\mathrm{v}$ batalione komandr. Bat. Gen. M. Ogarevym; [Medal]; Eia Imperatorskoe Velichestvo Gosudarynia Imperatritsa Mariia Feodorovna v sadu Kievskago dvortsa 23 marta 1917 g.; Polkovoe shit'e i tsvetushchii kaktus.

\section{Al'bom Kostromskoi gubernskoi zemskoi vystavki, ustroennoi} $v$ oznamenovie 300-lietiia tsarstvovaniia doma Romanovykh, v. $g$. Kostromie, $v 1913 \mathrm{~g}$.

Moscow: Fotografiia P.P. Pavlova, 1913. 60 leaves of plates, $35 \times 29 \mathrm{~cm}$. "Gimbel April 22, 1946" in pencil on first page. Cover hand-painted on white silk.

Presentation copy to Tsesarevich Aleksei Nikolaevich (1904-1918). "Arkhitektor L. R. Sologub, S. Peterburg" on each plate. Binder's title. Sologub, L. R. Fairs-Russia-Kostromskaia oblast. Exhibitions - Russia Kostroma.

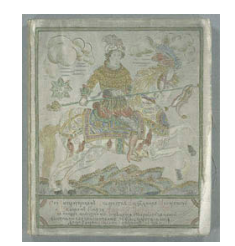

Slav. Reserve (Photo) (Albom Kostromskoi gubernskoi zemskoi vystavki) SLV 008 [Binder's title]; Tsarskii Pavil'on; Vnutrennii vid Tsarskago Pavil'ona; Tsarskiia Kresla raboty Vinogradovoi. Plafon Tsarskago Pavil'ona; Pavil'on Zemskago i Gorodskago khoziaistva; Vnutrennyi vid Zemskago Pavil'ona. Lestnitsa; Vnutrennii vid Zemskago Pavil'ona; Lesnoi Pavil'on T-va M-r Ivana Konovalova s synom; Vnutrennii vid Lesnogo Pavil'ona T-va M-r Ivana Konovalova s synom; Lesnoi Pavil'on; Vnutrennii vid Lesnogo Pavil'ona; Vnutrennii vid Lesnogo Pavil'ona; Pavil'on Krest'ianskago Pozemel'nago Banka. Kostromskoe Otdelenie; Vnutrennyi vid Pavil'ona Pozemel'nago Krest'ianskago Banka; Kostromskoe otdelenie; Ploshchad'; Obshchii Vid maloi ulichki; Obshchii vid ploshchadi; Pavil'on Sel'sko-Khoziaistvennyi; Vnutrennyi vid Sel'sko-Khoziaistvennago Pavil'ona; Navies dlia Sel'sko- 
khoziaistvennykh mashin i torzhok; Restoran; Vnutrennii vid Restorana. Lestnitsa na bashniu; Vnutrennii vid zdaniia Restoran-Teatra; Glavnyi vkhod; Ulitsa; Pavil'on Krupnoi Promyshlennosti; Vitrina Torgovago Doma Br. Kosatkinykh v Kostrome. Vitrina Torgovago n-tsy. Al'berta Gentse i Vasilii Kosatkin; Vnutrennii vid Pavil'ona Krupnoi Promyshlennosti; Vitrina T-go Doma M. N. Chumakova v g. Kostrome. Vitrina T-go Doma $\mathrm{N}$-tsy. I. Ia. Aristova v g. Kostrome; Vitrina T-vo Russkago BenzoloAnilinovago zavoda v gor. Kineshme Kostromskoi gub. Vitrina Kolbasnoi fabriki V. S. Golovanova S-vei v Kostrome. Vitrina I. V. Sokolova...; Pavil'on Tkatsko-Priadil'nyi; Vitrina T-va Nerekhtskoi M-ry K. A. Briukhanova. Vitrina Anonimnago obshchestva Kostromskoi M-ry Gratri Zherar i Mikhinoi; Obshchii vid ploshchadi; Obshchii vid ploshchadi; Pavil'on T-va Bol'shoi Kineshemskoi M-ry; Vnutrennii vid Pavil'ona T-va Bol'shoi Kineshemskoi M-ry; Pavil'on T-va M-ry osnovannykh I. I. Skvortsovym; Vnutrennii vid Pavil'on T-va M-r osnovannykh I. I. Skvortsovym; Pavil'on T-va Bol'shoi Kostromskoi L'nianoi M-ry; Vnutrennii vid Pavil'ona T-va Bol'shoi Kostromskoi L'nianoi M-ry; Pavil'on T-va l'nianoi M-ry Sosipatra Sidorova; Vnutrennii vid Pavil'ona T-va L'nianoi M-ry Sosipatra Sidorova; Vid s bashni restorana. Obshchii vid chastnye pavil'ony krupnoi promyshlennosti; Pavil'on T-va M-ry Anny Krasil'shchikovoi s synov'iami; Vnutrennii vid Pavil'ona T-va M-ry Anny Krasil'shchikovoi s synov'iami; Model fabriki T-va M-ry Anny Krasil'shchikovoi s synov'iami; Pavil'on T-va M-r Ivana Konovalova s synom; Vnutrennii vid Pavil'ona T-va M-r Ivana Konovalova s synom; Pavil'on T-va Bumago-Tkatskoi M-ry Br. G. i A. Gorbunovykh; Vnutrennii vid Pavil'on T-va M-ry Br. G. i A. Gorbunovykh; Zvonitsa Zavoda S. N. Zabenkina v Kostrome; Pavil'on T-va Visuchskikh M-r Br. F. i A. Razorenovykh; Vnutrennii vid Pavil'on T-va Visuchskikh M-r Br. F. i A. Razorenovykh; Pavil'on T-va M-r Gerasima Razorenova i Ivana Kokoreva; Vnutrennii vid Pavil'ona T-va M-r Gerasima Razorenova i Ivana Kokoreva; Bogatyr. Skul'ptor A. Kozel'skii; Ulitsa. Obshchii vid; Pavil'on Pivivarennago Zavoda "Novaia Bavariia" E. I. Kuni. Pavil'on Bulochnaia i konditerskaia S. Zablotskago v Kostrome; Beregovaia chast Vystavki; Vid na vystavku so storony ulitsy; Vid na Vystavku s vysoty.

\section{Al'bom ofitserskikh polkovykh grupp: Gvardeiskii korpus.}

[S.l.: s.n., 18-?] 27 mounted photographs, $32 \times 47 \mathrm{~cm}$. Title from original portfolio. Cover title. No text.

Twenty-seven uncaptioned plates on thick maroon mounts. Photographs are group portraits, each bears the stamp of N. Digo in lower right corner. Title reads: "Album of the officer regimental groups. Guards corps. Photographer of the troops of the Guards N. Digo."

Slavic Recon Project. Metropolitan New York Library Council, 2008. Russia. Armiia. Gvardeiskii Korpus. 
Slav. Reserve (Photo) (Albom ofitserskikh polkovykh grupp) SLV 009 Al'bom ofitserskikh polkovykh grupp. Gvardeiskii korpus. Fotograf voisk gvardii N. Digo. [Officers of Russian regimental groups]...

\section{Al'bom portretov byvshikh kadet: Pervyi i Kadetskago korpusa.}

St. Petersburg: Fototipiia V. Shteina i S. Lapteva, 1884. 3 p., 310 leaves of plates: ill., ports.; $30 \times 18 \mathrm{~cm}$. "1732-1863. An essay (16 p.)", "Pervyi kadetskii korpus, 1732-1863," by K. Volkhovskoi inserted. With bookplate of Grand Duke Vladimir Aleksandrovich. "Perl. Jun 18, 1931." See "spisok" inserted before page, indexed in alphabetical order by surname and date (portraits are arranged chronologically). Includes 310 portraits: 7 members of the imperial family, 303 former cadets.

Volkhovskii, K. Ia. Metropolitan New York Library Council, 2008. Slavic Recon Project. Pervyi kadetskii korpus (Russia) - Portraits. Pervyi kadetskii korpus (Russia) - History. Portraits, Russian.

Slav. Reserve (Photo) (Volkhovskii) SLV 010 Byvshikh kadet i kadetskago korpusa [Binder's title]; [cover title]; Ego

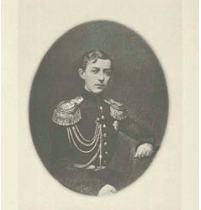
Imperatorskoe Velichestvo Gosudar' Imperator Aleksandr II; Ego Imperatorskoe Velichestvo Gosudar' Imperator Aleksandr III; Ego Imperatorskoe Vysochestvo Gosudar' Tsesarevich Nikolai Aleksandrovich; Ego Imperatorskoe Vysochestvo Gosudar' Velikii kniaz' Vladimir Aleksandrovich; Ego Imperatorskoe Vysochestvo Gosudar' Velikii Kniaz' Konstantin Nikolaevich; Ego Imperatorskoe Vysochestvo Gosudar' Velikii Kniaz' Nikolai Nikolaevich. Starshii; Ego Imperatorskoe Vysochestvo Gosudar' Velikii Kniaz' Mikhail Nikolaevich; General-maior Vladimir Petrovich Zhukovskii. 1797; General-adiutant Aleksandr Ivanovich Gil'denshtubbe. 1819; General-maior Ivan Pavlovich Markozov. 1819; General-maior Aleksandr Bogdanovich Dikheus. 1822; General ot infanterii Aleksiei Aleksievich Odintsov. 1823; General-maior Vladimir Vasil'evich Valoshinov. 1824; General ot kavalerii Aleksandr Fedorovich Baggovut. 1825; General-leitenant Nikolai Rustavovich Baggovut. 1827; Generalleitenant Pavel Petrovich Kinovich. 1827; General-adiutant Ivan Stepanovich Ganetskii. 1828; General-leitenant Karl Fedorovich Baggovut. 1828; Tainyi Sovetnik Aleksandr Petrovich Petrov. 1828; General-maior Fedor Vasil'evich Ustrugov. 1828; Polkovnik Aleksandr Kirilovich Shepetkovskii. 1828; General-maior Ivan Vasil'evich Kovalev. 1829; Generalleitenant Vasilii Ivanovich Firsov. 1829; General-maior Pavel Vasilevich Markovnikov. 1830; Tainyi Sovetnik Nikolai Ivanovich Ermolinskii. 1831; Baron Goiningen Gine. 1831; General-leitenant Miaskovskii. 1832; Generalot-infanterii Meletii Iakovlevich Ol'shevskii. 1833; General'nago shtaba. General-leitenant Vladimir Nikolaevich Matsnev. 1833; General-leitenant Aleksandr Petrovich Vikgorst. 1833; Deistv. Statsk. Sov. Konstantin 
Alekseevich Izmailov. 1833; General-leitenant Georgii Vasil'evich Pilenko. 1834; General-leitenant Aleksandr Ivanovich Savel'ev. 1834; Generalleitenant Aleksandr Germanovich Kleigil's. 1834; General-maior Petr Ivanovich Kononov. 1834; Mamert Gavrilovich Gordzialkovskii. 1834; General-ot-infanterii Nikolai Stepanovich Ganetskii. 1835; Otst. Generalleitenant Georgii Fedorovich Shostakov. 1835; General-leitenant Mikhail Andreevich Saranchov. 1836; Artillerii general-maior Konstantin Khristoforovich Ellerman. 1836; General-maior Aleksandr Fedorovich Geirot. 1836; Polkovnik Aleksandr Andreevich Afrosimov. 1836; Generalmaior Ferdinand Baumgarten. 1837; Polkovnik Nikolai Semenovich Gerakov. 1837; General-adiutant Aleksandr Romanovich Drentel'n. 1838; Voennyi inzhener general-leitenant Konstantin Ivanovich Ivanov. 1838; Generalleitenant Baumgarten. 1838; General'nago shtaba. Otst. General-leitenant Karl Andreevich Vil'k. 1838; General-maior Viktor Alekseevich Ivashchenko. 1838; General-maior Vladimir Gavrilovich Zuev. 1838; General-maior Mikhail Pavlovich Rozengeim. 1838; General-maior V. F. Vokach. 1838; General-maior Grigorii Vasil'evich Druzhinin. 1838; Statskii sovetnik Ivan Andreevich Tarasevich. 1838; General-leitenant Vasilii Emel'ianovich Savchenko-Bel'skii. 1839; Andrei Ivanovich Polonskoi. 1839; General-leitenant Porfirii Alekseevich Ivashchenko. 1840; Artillerii general-leitenant Sergei Ivanovich Shpakovskii. 1840; General-leitenant Iulii Karlovich Krit. 1840; Artillerii general-maior Dmitrii Iakovlevich Krasnoperov. 1840; General-maior Il'ia Vasil'evich Karpinskii. 1840; Polkovnik Aleksandr Vasil'evich Kozlov. 1840; Polkovnik Petr Danilovich Mein. 1840; Polkovnik Aleksandr Petrovich Lappa. 1840; Valerian Nikolaevich Vaksel. 1840; Arkadii Alekseevich Kolobov. 1840; Generalleitenant Zhukov. 1841; General-leitenant baron Viacheslav Vladimirovich Shteingel. 1841; Polkovnik Leonard Aleksandrovich Veinberg. 1841; Generalmaior Aleksandr Karlovich Krit. 1842; Polkovnik Andrei Ivanovich Ivanov. 1842; General-leitenant Vladimir Vasil'evich Notbek. 1843; General-leitenant Iakov Mikhailovich Kostogorov. 1843; General-leitenant Aleksandr Andreevich Barsov. 1843; General-maior Vasilii Mikhailovich Seliverstov. 1843; Deistvitel'nyi statskii sovetnik Nikolai Karlovich Krit. 1843; Generalleitenant Aleksandr Vasil'evich Prigorovskii. 1844; General-maior Valerian Dmitrievich Proskuriakov 2-i. 1884; Kamerger Dvora ego imp. Velichestva D. S.S. Petr Vladimirovich Zhukovskii. 1844; Ivan Aleksandrovich Kokushkin. 1845; Deistvitel'nyi statskii sovetnik Eduard Frantsovich von Shul'ts. 1845; Inzhener Konstantin Konstantinovich Girs. 1845; Kollezhskii sovetnik Nikolai Vladimirovich Zhukovskii. 1845; General-maior Apollinarii Emel'ianovich Fedorov. 1846; General-maior Nikolai Borisovich Fon Shveder. 1846; Polkovnik Konstantin Borisovich Shveder. 1846; Podpolkovnik Aleksandr Alekseevich Stepanov. 1846; Podpolkovnik Aleksandr Aleksandrovich Neidgart. 1846; General-leitenant Nikolai 
Nikolaevich Skvortsov. 1847; General-leitenant Pavel Pavlovich Kravchenko. 1847; Inzhener General-leitenant Georgii Ivanovich Shleifer. 1847; Kniaz' Dmitrii Semenovich Urusov. 1847; General-maior Vasilii Apollonovich Kakhanov. 1847; General-maior Aleksandr Vasil'evich fon Notbek. 1847; Dmitrii Aleksandrovich Roshtein. 1847; Poruchik Petr Alekseevich Liventsov. 1847; General-adiutant Nikolai Nikolaevich Obruchev. 1848; General'nago shtaba General-leitenant Nikolai Pavlovich Glinoetskii. 1848; General-maior Mikhail Timofeevich Ustrugov. 1848; General-maior Aleksandr Apollonovich Kirdan. 1848; General'nago shtaba General-maior Aleksei Afinigenivich Il'in. 1848; Artillerii Polkovnik Grigorii Grigor'evich Iavorskii. 1848; Polkovnik Georgii Markovich Al’bertov. 1848; Polkovnik Petr Vasil'evich Protopopov. 1848; Polkovnik Nikolai Aleksandrovich Dometi. 1848; Polkovnik Aleksandr Khrisoforovich Shtral'man. 1848; Polkovnik Bala-kishi-ali- bek Arablinskii. 1848; Polkovnik Gofman. 1848; Nikolai Fedorovich Shramm. 1848; Podpolkovnik Viktor Petrovich Kirilov. 1848; Podpolkovnik Mikhail Aleksandrovich Galberg. 1848; Konstantin Egorovich fon-Bool'. 1848; Maior Shakh-Nadir-Pirumov. 1848; General'nago shtaba General-leitenant Khristofor Khristoforovich Roop. 1849; General-leitenant Nikolai Karlovich Shvartsenberg. 1849; Generalleitenant Aleksandr Leont'evich Gagemeister. 1849; General'nago shtaba General-maior Aleksandr Ivanovich Lavrent'ev. 1849; General'nago shtaba General-maior Sergei Pavlovich Zykov. 1849; General-maior Kvitsinskii 1849; Polkovnik Nikolai Grigor'evich Nikiforov. 1849; Polkovnik Fon-Vite. 1849; Aleksei Efimovich Zhukov. 1849; Aleksandr Petrovich Khristiani I. 1849; Podpolkovnik Nikolai Fedorovich Nasekin. 1849; Maior Dmitrii Frantsovich Poplavskii 1849; Kapitan Mitrofan Ivanovich Samgin. 1849; General'nago shtaba general-leitenant Mikhail Fomich Petrushevskii. 1850; General-leitenant Petr Apollonovich Gresser. 1850; General-maior Semen Ivanovich Butenko. 1850; General-maior Antonin Fedorovich Pavlov. 1850; General-maior Nikolai Aleksandrovich Kolzakov. 1850; Deistv. Stat. Sovet. Nikolai Ivanovich Markov. 1850; Roty Ego Velichestva 4-go obshchago klassa Nikolai Andreevich Montanskii. 1850; General'nago shtaba General-maior Nikolai Ivanovich Krasnov. 1851; Svity Ego Velichestva General-maior baron Arpsgofen. 1851; General-maior Nikolai Aleksandrovich Lachinov. 1851; General-maior Aleksandr Ottovich Shmit. 1851; General-maior Vadim Vasil'evich Plaksin. 1851; Polkovnik Georgii Aleksandrovich Kushakevich. 1851; Polkovnik Aleksei Ivanovich Borislavskii. 1851; General-leitenant Aleksandr Karlovich Rikhter. 1852; General-leitenant Aleksandr Dmitrievich Goremykin. 1852; Dmitrii Dmitrievich Oblomievskii. 1852; General-maior Arkadii Aleksandrovich Khrushchov. 1852; General-maior Daniil Al'bertovich Konarzhevskii. 1852; General-maior Petr Nikolaevich Skvortsov. 1852; Polkovnik Il'ia Ivanovich Borislavskii. 1852; Polkovnik Vasilii Nikolaevich Kharlamov. 1852; Po gvardeiskoi kavalerii polkovnik 
Nikolai Andreevich Montanskii. 1852; Polkovnik Mikhail Mikhailovich Vendorf. 1852; Polkovnik Konstantin Vasil'evich Maslov. 1852; Podpolkovnik Georgii Vasil'evich Chishinich. 1852; Podpolkovnik Pavel Ivanovich Karnilovich. 1852; Vasilii Iustinovich Semenov. 1852; GeneralLeitenant Rengrikh Al'bertovich Konarzhevskii. 1853; General'nago shtaba. General-maior Ivan Ivanovich Ordynskii. 1853; General-maior Fedor Konstantinovich Al'bedil. 1853; General'nago shtaba. General-maior Konstantin Vasil'evich Rudanovskii. 1853; General-maior Aleksandr Aleksandrovich Kel'ner. 1853; Inzhener Konstantin Iakovlevich Mikhailovskii. 1853; Aleksandr Aleksandrovich Suslov. 1853; Kapitan Kokh. 1853; General-maior Aleksandr Nikolaevich Skvortsov. 1854; Deistv. St. Sovetnik Teterevnikov. 1854; Deistv. St. Sovetnik Vladimir Maksimovich Adlerberg. 1854; Statskii Sovetnik Mikhail Leont'evich Zlatkovskii. 1854; Polkovnik Nikolai Dmitrievich Safonov. 1854; Polkovnik Nikolai Nikolaevich Prokopovich. 1854; Artillerii-polkovnik Pavel Aleksandrovich Talantov. 1854; Polkovnik Mikhail Abramovich Sytenko. 1854; Fligeladiutant Polkovnik Andrei Aleksandrovich Nikitin. 1854; Polkovnik Vladimir Dmitrievich Merkazin. 1854; Podpolkovnik Tulatov. 1854; Kamerger dvora Ego Imper. Velichestva D.S.S. Konstantin Konstantinovich Sluchevskii. 1855; General'nago shtaba. General-maior Vladimir Nikolaevich Zubov. 1855; General-maior Aleksandr Karlovich Vodar. 1855; General'nago shtaba. General-maior Sergei Mikhailovich Dukhovskoi. 1855; General-maior Nikolai Il'ich Zhevanov. 1855; Deistv. Statsk. Sovetnik Ivan Borisovich fonShveder. 1855; Polkovnik Evgenii Fedorovich De-Roberti 1-i. 1855; Ieromonakh Afanasii 1855; Byv. Artillerii shtabs-kapitan Aleksandr Pavlovich Agafonov; Polkovnik Aleksandr Aleksandrovich Lishev. 1855; Polkovnik Eduard Eduardovich Bauman. 1855; Aleksandr Ivanovich Vidishev. 1855; Podpolkovnik Dmitrii Vladimirovich Potopchin. 1855; Kapitan Agafon Garsevanovich Kniaz' Vakhvakhov. 1855; General-maior Aleksandr Pavlovich Kobelev. 1856; Polkovnik Sigizmund Faddeevich Serzhpinskii. 1856; Otst. Polkovnik Ivan Fedorovich Zubarev. 1856; Polkovnik Vladimir Georgievich fon-Bool'. 1856; Polkovnik Vladimir Apolosovich Ivanov. 1856; Polkovnik Nikolai Nikolaevich Vishnevskii. 1856; Polkovnik Petr Timofeevich Zhegalov 1-i. 1856; Fligel-adiutant Polkovnik Ridiger. 1856; Polkovnik Aleksandr Nikolaevich Mokrinskii. 1856; Pavel Karlovich Rikhter. 1856; Polkovnik Vladimir Dmitrievich Tunoshenskii. 1856; Mikhail Iosifovich Musnitskii. 1856; Polkovnik Nikolai Fedorovich Glavatskii. 1856; Podpolkovnik Leonid Konstantinovich Sluchevskii. 1856; Svity Ego Velichestva General-maior Aleksei Gerasimovich Zherebkov. 1857; General-maior Ivan Fedorovich Tutolmin. 1857; General'nago shtaba. General-maior Vladimir Konstantinovich Andrievich. 1857; Inzhener Iulii Fedorovich Bal'ts. 1857; Aleksandr Vasil'evich Miagkov. 1857; Polkovnik Arkadii Aleksandrovich Engel'gardt. 
1857; Polkovnik Aleksandr Egorovich Lebedev. 1857; Konstantin Iakovlevich Volkhovskoi. 1857; Nikolai Dmitrievich Fefelov. 1857; Polkovnik Nikolai Nikolaevich Livotov. 1857; Polkovnik Kniaz' Georgii Evseevich Tumanov. 1857; Polkovnik Sergei Mikhailovich Trukhachev. 1857; Dmitrii Iakovlevich Kutuzov. 1857; Kapitan Vladimir Konstantinovich Volkov 1-I. 1857; Svity Ego Velichestva Gen. Sht. General-maior Nikolai Ivanovich Bobrikov. 1858; Ivan Fedorovich Oblomievskii. 1858; Aleksandr Karlovich Foigt. 1858; Polkovnik Petr Petrovich Tukmachev. 1858; Polkovnik Mikhail Vasil'evich Dzheneev. 1858; Pavel Nikolaevich Kozmin. 1858; Polkovnik Aleksandr Ivanovich Karnilovich 1858; Polkovnik Aleksei Konstantinovich Al'bedil'. 1858; Polkovnik Mikhail Medzhlumovich Shakh-Nazarov. 1858; Polkovnik Dmitrii Modestovich Rezvoi. 1858; Statskii Sovetnik Georgii Nikolaevich Gangardt. 1858; Polkovnik Aleksandr Emmanuilovich Ertel. 1858; Dmitrii Fedorovich Tutolmin. 1858; Maior Vladimir Pavlovich Rumiantsev. 1858; Nikolai Fedorovich Tutolmin. 1858; Inzhener Karl Karlovich Guber. 1859; Svity Ego Velichestva Gen. Sht. General-maior Georgii Ivanovich Bobrikov. 1859; General-maior Dmitrii Antonovich Skalon. 1859; General-maior Vasilii Fedorovich Argamakov 2. 1859; General'nago shtaba Polkovnik Aleksandr Fedorovich Bal'ts. 1859; Polkovnik Baron Kridener. 1859; Polkovnik Dmitrii Timofeevich Svishchevskii. 1859; Polkovnik Aleksei Iosifovich Dzichkanets. 1859; Polkovnik Nikolai Mikhailovich Bez-Kornilovich. 1859; Polkovnik Mikhail Vladimirovich Miller. 1859; Podpolkovnik Aleksandr Ivanovich Shipunov. 1859; Statskii Sovetnik Nikolai Aleksandrovich Lishev. 1859; Polkovnik Aleksandr Ivanovich Sitnikov. 1859; Otst. Artilleriipodpolkovnik Nikolai Abramovich Sytenko. 1859; Polkovnik Fedor Ivanovich Vol'kenau. 1859; Kapitan Konstantin Aleksandrovich Engel'gardt. 1859; Nikolai Aleksandrovich Perren Sinel'nikov. 1859; Dmitrii Viktorovich Pokatilov. 1860; Aleksandr Grigor'evich Voronov. 1860; Isidor Petrovich Boreisha. 1860; Polkovnik Viktor Ivanovich Kosinskii. 1861; Podpolkovnik Karl Moritsovich Fon-Gagman. 1861; Kapitan Anatolii Fedorovich Trusov. 1861; General-maior Nikolai Venediktovich Bogaevskii. 1862; Voennyi inzhener Polkovnik Viktor Alekseevich Prokhorov. 1862; Polkovnik Timofei Mikhailovich Beliaev. 1862; Polkovnik Vladimir Ivanovich Amosov. 1862; Artillerii polkovnik Konstantin Frantsovich Vitkovskii. 1862; Adiutant Moskovskago general-gubernatora Aleksandr Nikolaevich Vishnevskii. 1862; Podpolkovnik Aliev. 1862; Voiskovoi starshina Khristofor Fomich Makovskii. 1862; Polkovnik Mikhail Illarionovich Markov. 1862; Polkovnik Ivan Vladislavovich Gorev. 1862; Podpolkovnik Viktor Nikolaevich Pashchenko. 1862; Aleksandr Aleksandrovich Kushakevich. 1862; Orest L'vovich Semenov. 1862; Inzhener Andrei Mikhailovich Voznesenskii. 1862; Maior Fedor Fedorovich Orel. 1862; General-maior Leonid Nikolaevich Sobolev. 1863; General'nago shtaba polkovnik Aleksandr Kazimirovich Puzyrevskii. 1863; Konnoi Artillerii 
Podpolkovnik Vladimir Nikolaevich Baggovut. 1863; Polkovnik Aleksei Filippovich Kudriavtsev. 1863; Polkovnik Fedor Aleksandrovich FonNandel'shchtedt. 1863; Polkovnik Nikolai Vladimirovich Shcherbakov. 1863; Voennyi inzhener-polkovnik Kniaz' Nikolai Evseevich Tumanov. 1863; General'nago shtaba Polkovnik Nikolai Nikolaevich Sukhotin. 1863; Polkovnik Pakhalen 3-ii. 1863; Polkovnik Nikolai Nikolaevich Maslov. 1863; Polkovnik Petr Fomich Luzanov. 1863; Podpolkovnik Petr Aleksandrovich Stakhiev 2-i. 1863; Podpolkovnik Konstantin Aleksandrovich Dykhov. 1863; Kapitan Gvardeisk. Artil. Konstantin Aleksandrovich Ogil'vi. 1863; Artilleriikapitan Aleksandr Aleksandrovich Petrov. 1863; Artillerii kapitan Viktor Ivanovich Karpov 2-i. 1863; Aleksei Georgievich Gebel. 1863; Rotmistr Al'fred Konstantinovich Baron fon-Tizengauzen. 1863; Aleksei Nikolaevich Sukhotin 2. 1863; General'nago shtaba General-maior Aleksei Nikolaevich Kuropatkin. Kapitan Aleksei Dmitrievich Merkazin. 1864; Nikolai Epaminondovich Gaius. 1863; Polkovnik Aleksandr Karlovich Birger. 1863; Polkovnik Viktor Ignat'evich Zhiliai. 1863; Polkovnik Rostislav Antonovich Reinbot. 1863; Podpolkovnik Nikolai Aleksandrovich Enosko-Borovskii. 1863; Podpolkovnik Vladimir Gavrilovich Zubinskii. 1863; Podpolkovnik Mikhail Venediktovich Bogaevskii. 1863; Podpolkovnik Pavel Petrovich Makhaev. 1863; Podpolkovnik Dukmasov. 1863; Kapitan Petr Nikolaevich Lebedev. 1863; Kapitan Aleksandr Aleksandrovich Shepetkovskii. 1863; Kapitan Fedor Liudvigovich von-Kliman. 1863; Artillerii kapitan Roman Karlovich Birger. 1863; Kapitan Fedor Petrovich Falenberg. 1863; Georgii Ivanovich Strazhevskii. 1863; Shtabs-kapitan Aleksandr Vasil'evich Osipov. 1863; Kapitan Iosif Aleksandrovich Shendzikovskii 1-yi. 1863; Inzhenertekhnolog Mikhail Aleksandrovich Shendzikovskii 2-i. 1863; Kniaz' Zakharii Aleksandrovich Dzhordzhadze. 1863; Nikolai Mikhailovich Kudriavtsev. 1863; Konstantin Fedorovich Maslovskii. 1863; Poruchik Il'ia Nikolaevich Khristoforov. 1863

Al'bom snimkov drevnostei i dostoprimiechatel'nostei, khraniashchikhsia vriznitsie Arkhiereiskago doma v Iaroslavlie

Yaroslavl: Fotografiia V. A. Lopatina, [18-?]. 30 mounted photographs, $31 \times 26 \mathrm{~cm}$.

Title supplied by cataloger. Album of photographs with descriptive letterpress, originally in box. Thirty photographs of treasures from the SpasoPreobrazhenskii monastery and Archbishop's House in Iaroslavl. Includes one general view of the monastery, many plates of icons, textiles, and elaborately decorated crosses and other articles. Album of photographs of antiquities and objects of distinction kept in the treasury of the Archbishop's House in Iaroslavl.

Slavic Recon Project. Metropolitan New York Library Council, 2008. Iaroslavskii Spaso-Preobrazhenskii monastyr'.

Church decoration and ornament- Russia (Federation) - Iaroslavl (Iaroslavskaia oblast). Icons, Russian -Russia - Iaroslavl (Iaroslavskaia oblast). 


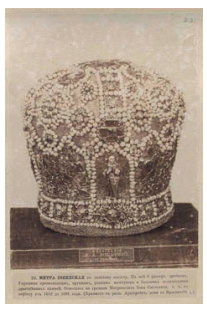

Slav. Reserve Photo (Albom snimkov drevnostei...) SLV 011 Spaso-Preobrazhenskii Monastyr'...; Vkhodnaia dver' v khram preobrazheniia Gospodnia...;; Ikona Gospoda Savaofa....; Ikona- Spasitelia...; Ikona-Simeona Bogopriimtsa...; Drevneishie kresty i panagii...; Drevneishie kresty i panagii....; Krest vynosnyi...; Krest naprestol'nyi....; Darokhranitel'nitsa....; Plashchanitsa....; Vozdukh....; Sakkos....; Sakkos...; Palitsy...; Epitrakhili...; Panagiia....; Panagiia....; Panagiia....; Panagiia....; Posokhi; Mitra...; Mitra Ioninskaia...; Klobuk mitropolichii...; Evangelie...; Umyval'nitsa...; Chasha...; Sosudtsy...; Bliudo...; Slovo postnicheskoe...

\section{Al'bom suvorovskikh polei srazhenii.}

Warsaw: A. Khudovetskii, 1900. 67 photographs on 17 leaves, $47 \times 34 \mathrm{~cm}$. Stamped "Perlstein Jun 18 1931." At head of title: “1729- 1800.” Inserted: "Kratkoe opisanie snimkov na Suvorovskikh Poliakh Srazhenii" (brief descriptions of battles fought by Suvorov, with maps). Sixty-seven photographs, mainly of importance related to battles and events in 1769-1772 and 1794. It has a gold embossed leather binding with a metal clasp (intact), thick gilt-edged plates in which photographs are mounted, and silk moiré endplates. The first photograph is in a hand painted mount.

Metropolitan New York Library Council, 2008. Slavic Recon Project. Khudovetskii, A. Suvorov, Aleksandr Vasil'yevich, knyaz', 1730-1800. Russia-History, Military-To 1801.

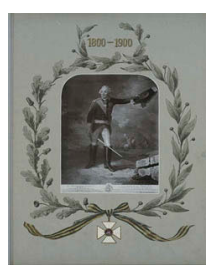

Slav. Reserve (Photo) (Albom Suvorovskikh polei srazhenii) SLV 012 [Photograph of a engraving of Suvorov, dedicated to emperor Paul I]; Selo Oriekhovo/Poliana $\mathrm{v}$ urochishchie Kryvna; Front nastupleniia russkikh/Poliana s mogiloi konfederatov; Urochishche Bruski/Miestechko Stolovichi; Tserkov' i ploshchad' v Stolovichakh/Greblia i ulitsa, po kotorym russkie vorvalis' v Stolovichi; Greblia pri v'ezdie v Stolovichi iz goroda Mira/Dom, postroennyi na mogilie pavshikh voinov; Stolovichskie starozhily/Imienie "Guberniia" gde zhil Suvorov; Prud v imienii "Guberniia"/Suvorovskaia ulitsa; Suvorovskii pereulok/Petro-Pavlovskaia tserkov', nazyvaemaia "Suvorovskoi"; Vnutrennii vid nynieshnei "Suvorovskoi" tserkvi/Staryia ikony "Suvorovskoi" tserkvi; Staryia ikony "Suvorovskoi tserkvi"/Miestnost' boia 4-go Sentiabria 1794 g.; Ostatki Krupchitskago monastyria/Tserkov" v Krupchitskakh; Ikonostas Krupchitskoi tserkvi/Prodol'nyi vid pol'skoi pozitsii; Plotina cherez $\mathrm{r}$. Trostianitsu/Vid s pozitsii, gdie stoiala russkaia batareia; Miesto perepravy russkoi piekhoty cherez r. Trostianitsu/Miesto perepravy pravoi obkhodnoi kolonny generala Shevicha; Miesto perepravy Suvorova cherez r. Bug/Mogila russkikh voinov; Krest'ianin d. Bolotkov, ukazavshii mogilu/Pol'skaia pozitsiia u d. Koroshchin; Koroshchinskaia tserkov'/Plotina cherez r. Kginu; Tserkov' v d. Dobrin/Miesto perepravy 
Generala Ferzena cherez r. Vislu; Vargotsinskii Kostel/Miestechko Matsievitse; Vid zamka/Lipa u zamka; Miesto zakhvata Kostsiushki/ Paviarnia v Podzamcheskom parkie; Tsentr pol'skoi pozitsii/Lievyi flang pol'skoi pozitsii; Put' otstupleniia poliakov/Mogily russkikh voinov; Front nastupleniia kolonny Denisova/Kostsiushko v boiu pod Matsievitsami; Derevnia Kobylka/Pozitsii poliakov na opushkie liesa; Vysota kuda otstupali poliaki/Miestnost' "Virshkovisk", gdie byli plieneny poliaki; Dom, gdie Suvorov obiedal s russkimi i pliennymi pol'skimi ofitserami/Varshava i Praga v 1770 godu; Lievyi flang pol'skikh ukreplenii/Miesto, gdie Suvorov nabliudal khod shturma; Peredovyia pol'skiia ukriepleniia/Pol'skiia ukriepleniia vostochnago fasa; Stienka evreiskago kladbishcha v iskhodiashchem uglu pol'skikh ukreplenii/Stienka kladbishcha s vostochnoi storony; Rukav Visly, gdie byl most, po kotoromu nastupala kolonna gen. Denisova/Visla v tom mieste, gdie byl most; Dom, gdie byla glavnaia kvartira Suvorova/Dom, gdie byla pokhodnaia tserkov' Suvorova; Vnutrennii vid komnaty, gdie byla tserkov' Suvorova/Mostovaia ulitsa

[Al'bom vidov Solovetskogo monastyriai snimkov drevnostei i dostoprimechatel'nostei, khraniashchikhsia $v$ ee riznitse].

[Russia?: s.n., 18-?]. 48 mounted albumen photographs, $24 \times 31 \mathrm{~cm}$. Hand written captions; originally in portfolio. Slavic Recon Project.

Metropolitan New York Library Council, 2008. Solovetski monastyr'. Monasteries, Orthodox Eastern -Ukraine-Kiev. Monasteries, Orthodox Eastern -Russia. Religious articles- Russia.

Slav. Reserve (Photo) (Albom vidov Solovetskogo monastyria) SLV 013 Naberezhnaia Solovetskago monastyria; Rybatskaia pristan' u morskago zaliva bliz Solovetskago monastyria; Vid Solovetskago monastyria s vostochnoi storony; Vid Solovetskago monastyria s zapadnoi storony; Chasovnia Sviatitelia Filippa; Anzerskii skit na Solovetskom ostrovie; Chasovnia Zameniia Bozhiei Materi v Anzerskom skitu; Savvatievskii skit na Solovetskom ostrovie; Dereviannyi dom gostinnitsy, prostrielennyi anglichanami v 1854 godu; Biologicheskaia stantsiia na Solovetskom ostrovie; [Historical plaque]; [Historical plaque]; Zapis' Marfy Posadnitsy na imia Prepodobnago Zosimy Solovetskago; [Historical plaque]; [Historical plaque]; [Historical plaque]; Evangelie XVI stolietiia; Evangeliia XVI stolietiia; Evangeliia XVI stolietiia; Evangelie, pozhertvovannoe mitropolitom Novgorodskim Isidorom v 1609 godu; [Ornate binding]; Obraz Sviatitelia Filippa; [Ornate binding]; Zolotye kresty tsaria Ianna Vasil'evicha Groznago s moshchami sviatykh; Kresty XVI stolietiia; Kadila Sviatitelia Filippa; Dva kadila igumena Filippa; Dereviannyi potir' Prep. Zosimy; Potir' i bliudo Sviat. Filippa; Zolotoi potir' Tsaria Mikhaila Feodorovicha; Potir' i kovsh serebrianye, pozhertvovan. Tsarem Mikhailom Feodorovichem v 1638 godu; Kubki, pozhalovannye v 1583 g. tsarem 
Ioannom Vasil'evichem Groznym; [Ladles donated by Peter I]; [Ladles donated by Peter I]; [Bell]; [Bell from the Solovetskii monastery]; [Bell]; Mitra, pozhalovannaia tsaritseiu Praskov'ei Feodorovnoi; Riza igumena Filippa; Riza igumena Filippa; Pokryshka s shuby Sviatitelia Filippa (1566 goda); Riza Prepodobnago Zosimy; Pelena i vozdukhi, pozhalovannye tsarem Mikhailom Feodorovichem; Vozdukh i pokrovtsy, pozhalovannye tsarem Mikhailom Feodorovichem v 1630 godu; [Religious object]; [Religious object]; Kruzhka iz tsiel'nago morzhovago zuba, raboty krest'ianina Osipa Dugina, 1774 goda; Palagi' kniazia Mikhaila Vasil'evicha Shuiskago-Skopina i sablia kniazia Dmitriia Mikhailovicha Pozharskago

Al'bom vidov tserkvei Estliandskoi gubernii/ sooruzhennykh pod viedieniem Revel'skago Nabliudatel'nago Komiteta po postroikie tserkvei prichtovykh i shkol'nykh zdanii.

[S.l.: s.n.], [1889?]. 19 mounted photographs, $43 \times 35 \mathrm{~cm}$. In a leather portfolio, with the crowned gilt monogram of Alexander III, Emperor of Russia (r. 18811894). Fekula Collection. Shirinskii-Shikhmatov, A. A. DOE Title II-C "Russian Illustrated Books and Photographs" Project. Church buildings - Estonia.

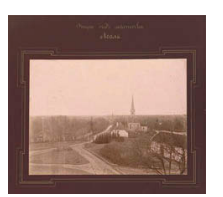

Slav. Reserve (Photo) 91-6302 SLV 014 [Title page]; Tserkov' Rozhdestva Khristova Novo-Verderkago prikhoda; Vnutrennii vid tserkvi Sv. Blagoviernago Velikago Kniazia Aleksandra Nevskago Leal'skago prikhoda; Obshchii vid miestechka Leal'; Tserkov' Sv. Blagoviernago Velikago Kniazia Aleksandra Nevskago Leal'skago prikhoda; Obshchii vid postroek Vellisskago prikhoda; Tserkov' Sv. Ioanna Predtechi Velliskago prikhoda; Vnutrennii vid tserkvi Sviatago Ioanna Predtechi Vellisskago prikhoda; Tserkov' Rozhdestva Presviatyia Bogoroditsy Oleshnitskago prikhoda;

Vid prezhniago molitvennago doma Viaike-Lakhtrskago prikhoda; Obshchii vid postroek Viaike-Lakhtrskago prikhoda; Tserkv' Rozhdestva Presviatyia Bogoroditsy Viaike-Lakhtrskago prikhoda; Vnutrennii vid tserkvi Rozhdestva Khristova Novo-Verderskago prikhoda; Vnutrennii vid tserkvi Sviatyia Troitsy Ladenormskago prikhoda; Tserkov' Sviatyia Troitsy Ladenormskago prikhoda; Tserkov' Vozneseniia Gospodnia Liukhalepskago prikhoda (na ostrovie Dalo.); Tserkov' Rozhdestva Presviatyia Bogoroditsy Emmastskago prikhoda (na ostrovie Dalo.); Obshchii vid postroek Emmastskago prikhoda (na ostrovie Dalo.); Vnutrennii vid tserkvi Vormskago prikhoda (na ostrovie Vorms.); Ikona Sviatago Blagoviernago Velikago Kniazia Aleksandra Nevskago

\section{Al'bom vidov Uspenskoi Kievo-Pecherskoi Lavry i snimkov drevnostei $i$ dostoprimiechatel'nostei, khraniashchikhsia $v$ eia riznitsie.}

[S. l.: s. n., 1875?]. 213 mounted photographs on 94 plates. $39 \times 30 \mathrm{~cm}$. Cover title. Captions on each plate. From cover of box in which the album was originally issued. 
Metropolitan New York Library Council, 2008. Slavic Recon Project. KyievoPecherska lavra. Monasteries, Orthodox Eastern -Ukraine -Kiev.

Slav. Reserve (Photo) (Albom vidov Uspenskoi Kievo-Pecherskoi lavry) SLV 015 [Cover title]; Obshchii vid Lavry s iuzhnoi storony; Vid Lavrskoi sobornoi tserkvi s Zapadnoi storony; Vid Lavrskoi drevnei sobornoi tserkvi s zapadnoi storony; Vid sv. vorot s zapadnoi storony/Vid sv. vorot s vostochnoi storony; Vid Lavrskoi sobornoi tserkvi s kolokol'neiu s Zapadnoi storony; Vid ekonomicheskikh vorot s sievernoi storony; Obshchii vid Blizhnikh i Dal'nikh peshcher s sievernoi storony; Vid Sviato-Preobrazhenskoi kladbishchenskoi tserkvi s zapadnoi storony; Vid Golosieevskoi pustyni s iuzhnoi storony; Vid Kitaevskoi pustyni s vostochnoi storony; Zhezl' fel'dmarshal'skii, zolotoi/Trost' kamyshevaia s serebrianoiu rukoiatkoiu i finiftovymi na nei izobrazheniiami i chasy; [Lampady i kadilo]; [Epitrakhili i orari]; [Poruchi]; [Omofori]; [Darokhranitel'nitsy]; [Darokhranitel'nitsy]; [Palitsy]; [Palitsy i pokrovy]; [Mitry]; [Mitry]; [Mitry]; [Panagii]; [Panagii]; [Panagii]; [Krugi iz miednykh listov]; [Ikona Sviatago Velikago kniazia Vladimira/Ikona Bogomateri]; [Ikona velikomuchenitsy Varvary/Ikona Presviatyia Troitsy/Ikona Bogomateri]; Starinnyia ikony: Bogomateri/Spasitelia Deisus/Ioanna Predtechi; [Ikona Spasitelia/Ikona Bogomateri/Ikona Spasitelia]; [Ikona Uspeniia Presviatyia Bogoroditsy]; [Ikona - boliashchii Iosif/Ikona Spasitelia]; [Ikona Sviatitelia Nikolaia/Ikona - sniatie Spasitelia so kresta]; [Molitvoslov Vilenskoi pechati 1695 goda/Chasy/Vozdukhi drevnie]; [Chasha zolotaia/Diskos i zviezditsa/Diskos]; [Chasha srebropozlashchennaia/Diskos/Diskos]; [Bokal/Bokal/Kubok]; [Chasha zolotaia/Diskos zolotoi/Diskos]; [Chasha zolotaia/Diskos zolotoi/Diskos]; [Chasha/Diskos/Diskos]; [Kovsh/Bliudo/Tarelka/Panagiar]; [Kruzhka/Steklianye bokaly, solonki, i sakharnitsa]; [Kovshi, bokaly, kruzhki, chainiki, solonki, kubki, i stakany]; [Chashi, zviesditsa, lzhitsy]; [Bokaly i kubok]; [Kovshi]; [Taz i kruzhki]; [Zhezl', podsviechniki, krest, i alavastr]; [Chasha serebrianaia dlia osviashcheniia vody]; [Pokrov' zolotago glazeta]; [Parchevyi pokrov' na sviatyia moshchi]; [Plashchanitsa, shitaia zolotom i serebrom]; [Plashchanitsa]; [Plashchanitsa vsia shitaia zolotom]; [Evangelie starinnoe, 1538 goda]; [Evangelie]; [Evangelie v srebropozlashchennykh doskakh]; [Evangelie v miednopozlashchennom okladie]; [Evangelie v srebropozlashchennom okladie]; [Evangelie Kievskoi pechati 1865 goda]; [Evangelie v barkhatie]; [Evangelie serebrianoe]; [Evangelie starinnoe, rukopisnoe, v barkhatie]; [Evangelie Moskovskoi pechati 1681 goda]; [Evangelie $\mathrm{v}$ barkhatie s nakladnymi srebropozlashchennymi izobrazheniiami]; [Evangelie srebropozlashchennoe s izobrazheniiami chekannoi raboty]; [Evangelie v zolotoi i srebropozlashchennoi doskakh, s dragotsiennymi kamniami]; [Mantiia/Sakkos serebrianoi parchi v zolotykh i shelkovykh tsvietakh]; [Sakkosy]; [Riza so stikharem zolotoi parchi v shitykh shelkom rozakh]; [Riza so stikharem serebrianoi parchi po krasnomu atlasu]; [Rizy zolotoi parchi]; [Riza krasnago barkhata/Riza 
zelenago venetsianskago barkhata]; [Riza zelenago barkhata/Riza venetsianskago krasnago barkhata]; [Riza i stikhar' neshveinye iz odnago kuska golubago rytago barkhata]; [Riza i stikhar' golubago krestovago barkhata po zolotomu poliu]; [Riza i stikhar' serebrianoi parchi]; [Riza i stikhar' starinnoi bogatoi zolotoi parchi v dragotsiennykh kamniakh]; [Riza zolotoi parchi/Riza bielago shelku]; [Riza so stikharem serebrianoi parchi s zolotymi i shelkovymi tsvietami]; [Riza so stikharem zolotoi parchi po golubomu poliu]; [Riza serebrianago glazeta s zolotymi tsvietami/Riza parchevaia s zolotymi rozvodami]; [Riza vishnevago granitura/ Stikhar' serebrianoi parchi]; [Gramota Gosudaryni Imperatritsy Ekateriny II o proizvodstvie ieromonakha Zosimy Val'kevicha v arkhimandrita]; Gramota Gosudaryni Imperatritsy Elisavety Petrovny s podtverzhdeniem drevnikh prav stavropigii i privillegii Kievo-Pecherskoi Lavry ot 10 Avgusta 1752 goda; Gramota Gosudaryni Imperatritsy Elisavety Petrovny s podtverzhdeniiem prav stavropigii Kievo-Pecherskoi Lavry ot 3 Noiabria 1742 goda; Gramota Gosudaryni Imperatritsy Anny Ioannovny o naznachenii arkhimandrita KievoMikhailovskago monastyria Timofeia Shcherbatskago arkhimandritom KievoPecherskoi Lavry ot 24 Sentiabria 1740 goda; Zhalovannaia Kievo-Pecherskoi Lavrie gramota Gosudaria Imperatora Petra I na pravo stavropigii, pisannaia na pergamentie ot 16 Oktiabria 1720 goda; [Kresty]; [Kresty]; [Kresty]; [Kresty]; [Kresty]; [Kresty]

\section{Al'bom vnut. vidy Remeslennoi vystavki/Vidy sniaty fotografom \\ S. Rubinovichem.}

St. Petersburg, 1885. 34 mounted photographs, 34 x $27 \mathrm{~cm}$. Rubinovich, S. (Photographer). Canvas bound album of 34 albumen prints, mounted one per page. Title on cover in gold on red canvas. The first page has a dedication written in ink to the Grand Duke Vladimir Aleksandrovich, signed by the photographer, identifying the exhibition as "the St. Petersburg Arts and Crafts Exhibition, with others on the Nevskii Prospekt," no. 52, St. Petersburg, dated 23 May 1885 (labels with the photographer's name and address appear on verso of some of the pages). The inside back cover has a red stamp: May 24, 1934.

Documentation on various booths and displays: taxidermists, cabinetmakers (several plates), metalwork, glass, electrogalvanization, uniforms, carriages, tents, flowers, etc. On verso pp. 8-9: work of the artistic school founded in memory of Tsarevich Nicholas. A collection of unremarkable images except for one interesting product: p. 13, a chair made out of peasant tools.

Photographs are brittle mounts with covers missing. DOE Title II-C "Russian Illustrated Books and Photographs" Project. Carriages \& coaches Russia - Exhibitions. Decorative arts - Russia - Exhibitions. FurnitureExhibitions-Russia. Carriages and carts-Exhibitions-Russia. 


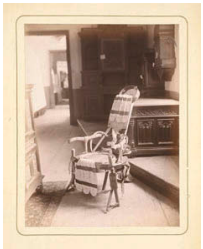

Slav. Reserve (Photo) (Albom Vnut.) SLV 016 [Cover title]; [Statue of Alexander II]; [Inside exhibition of taxidermied wild animals]; [Modern window frame and a desk by a cabinet maker]; [Interior view of the exhibition]; [A perspective view inside the exhibition]; Pamiatnik v g. Astrakhanie; [View of stands of furniture]; [Stand with mirrors and furniture]; [Stand with different tools and machinery]; [Stand with oil lamps, birdcages, and other artifices]; [Stand with bathroom fixtures and bathtubs in different shapes]; [Stand with furniture]; [Armchair]; [Stand with furniture]; [Stand of I. Peterson]; [Stand with furniture]; [A hexagonal cabinet of flowers and jars]; [Stands with bottles, condiments, and small folding chairs]; [Stand of Afanasiev]; [Several small glass stands]; [Stand of decorations and metal plated objects]; [A hexagonal cabinet filled with gloves]; [Stand of carriages]; [Stand of carriages]; [Stand of wrought iron works]; [Stand with croquet game parts]; [A model of His Imperial Highness's tent]; [Stand of accounting company F. V. Ezerskov]; [Display of different lace objects]; [Display of taxidermied animals and birds]; [Statue of Aleksandr Pushkin]; [Greenery and flowers]; [Display of flowers by M. P. Popov]; [Display of liquor by the Bekman company]

\section{Al'bom voenno-pokhodnoi svietopisi Rushchukskago Otriada.}

[S.l.: s.n., 1878?] 76 photographs: ill., map (folded), plans; 30 x 24 x $33 \mathrm{~cm}$. Cover title: Ego Imperatorskomu Vysochestvu Gosudariu Velikomu Kniaziu Vladimiru Aleksandrovichu v pamiati Vostochnoi voiny 1877-78 g.

Captions in manuscript. Caption on the plan reads: "Pamiati voiny 18771878." With bookplate of Grand Duke Vladimir Aleksandrovich.

Metropolitan New York Library Council, 2008. Slavic Recon Project. Russia. Armiia - Maneuvers. Russo-Turkish War, 1877-1878 -Campaigns - Bulgaria Maps. Russo-Turkish War, 1877-1878 - Campaigns Bulgaria. Ruse (Bulgaria).

Slav. Reserve (Photo) (Albom voenno pokhodnoi svietopisi Rushchukskago otriada) SLV 017 [Cover title page]; [Map]; Opyty primieneniia svietopisi $\mathrm{k}$ voennym tsieliam; Dvorets E. I. V. G. Vl. Kn. Nasliednika Tsesarevicha v s. Brestovetsie; [Group of soldiers]; [Group of soldiers standing]; Byv. Kv. Ego Imp. Vys. Gosud. Vl. Kn. Sergieia Maksimilionovicha v s. Brestovetsie; Prikhodskaia Tserkov'v s. Brestovetsie; [Housing]; Kvartira L. Md. Ego Prvekh. Girsha v s. Brestovetsie; Kvartiry Sv. Kn. Imeritinskago i Kapitana Stepanova v s. Brestovetsie; [House with soldiers sitting outside]; Khudozhnik V. Dm. Polienov za rabotoiu u svoei kvartiry v s. Brestovetsie; Kvartira E. I. V. Vl. Kn. Nasliednika Tsesarevicha v s. Brestovetsie s okrestnymi stroeniiami; Tipy zhilishch' v s. Brestovetse; Komendant Rushchukskago Otriada so shtabom u svoei kvartiry v s. Brestovetsie; Raketnaia i skorostriel'naia Morskaia batareia, sostoiavshaia pri Sht. Rushch. Otriada v s. Brestovetsie; [Housing]; 
Stolovaia prisluga Ego Imper. Vysoch. Gosud. Nasl. Tsesarevicha v c. Brestovetsie; Markitantskaia lavka Sht. Rushchuks. Otriada v s. Brestovetsie; S. Brestovets. Raskvartirov Sht. Rushchuk. Otr. (Dvortsovaia chast'); S. Brestovets. Raskvart. Sht. R. Otriada (Komendantskaia chast'); [Group of soldiers]; [Clergyman and Russian officers]; [Clergyman and soldiers]; Kvartira E. I. V. Gs. Vl. Kn. Vladimira Aleksandrovicha v S. Iali Ablanovo; Prikhodskaia tser'kov' v s. Iali-Ablanovo; Nachal'nik shtaba 12 Ar. Korp. G. M. Kosich so Shtabom u svoei kvartiry v s. Iali Ablanovo; Shtab 12 Armeiskago Korpusa (v s. Iali Ablanovo); Stolovaia E. I. V. G. Vl. Kn. Vladimira Aleksandrovicha v s. Iali Ablanovo; Kvartira Gvardii Kapitanu Ivkova v s. Iali Ablanovo; Boevye koni E. I. Vys. Gos. Vl. Kn. Vladimira Aleksandrovicha pri Koniushni v s. Iali Ablanovo; Stolovaia prisluga Ego Imperatorskago Vysochestva Gos. Vl. Kn. Vladimira Aleksandrovicha v s. Iali Ablanovo; Narodnye tipy Bolgarii; Narodnye tipy Bolgarii; Narodnye tipy Blgarii. Gulianie u dvortsa E. Imp. V. Vl. Kn. Vladimira Aleksandrovicha v s. Iali Ablanovo; Rushchuk. U pristani na beregu r. Dunaia; Rushchuk. Vid na beregovye ukriepleniia na r. Dunai; Ust'e rieki Loma; Rushchuk. Batareia na uglu kriepostnoi ogrady; Kr. Rushchuk. Turetskaia "bombovaia pushka"vooruzh. batarei protiv S. Slobodzei (chrez. r. Dunai); Kr. Rushchuk. Turetskii "edinorog"-vooruzhenie beregovykh batarei protiv s. Slobodzei (chrez r. Dunai); Kr. Rushchuk. Kruppovskoe stal'noe orudie; Kr. Rushchuk. Turets. beregovaia batareia, v kriepostnoi ogradie Rushchuka; Kr. Rushchuk. Tyl'nyi (sever.) fas forta Leventabiia/Kr. Rushchuk; Kr. Rushchuk. 15 santim. Kruppovskoe stal'noe orudie na fortie Leventabiia; Kr. Rushchuk. Turets. Mortira i kriep. pushka - na fortie Leventabiia; Vid na bereg r. Dunai u v'ezda na plotovoi most; Plotovoi most chrez r. Dunai ot Rushchuka k Zhurzhevo; Prervannaia pereprava u Rushchuka chrez r. Dunai; Podvizhn. lazaret 33i pekh divizii pod kr. Rushchukom; Tipy g. Rushchuka; Tipy g. Rushchuka; Tipy g. Rushchuka; Narodnye tipy; G. Rushchuk. Po konsul'skomu pereulku; G. Rushchuk. Po konsul'skomu pereulku, k pristani; Tipy i stseny Rushchuka; Tipy i stseny Rushchuka; Blgarskie tipy i steny; [Group of soldiers]; [Group of soldiers with horses]; [Group of soldiers]; [Group of soldiers]; [Group of soldiers]; [Residents of Iali-Ablanovo]; [House for Russian soldiers at Iali-Ablanovo]; [House for Russian soldiers at IaliAblanovo]; [Russian officers]; [Clergyman and soldiers]; [Clergyman and soldiers]; [Group of soldiers]; [Local residents]; [House for soldiers]; [House for soldiers]; [Group of soldiers]; [Dancing local residents]; [Residents of Iali-Ablanovo].

[Album of photographs of landscapes around Lankorona-Izdebnik presented to the Archduke of Austria, Rainer (1827-1913) on January 11, 1897]. (1906). 14 mounted photos, $40 \times 47 \mathrm{~cm}$.

Album of 14 photographs taken by Awit Szubert of landscapes around Lanckorona-Izdebnik (nearby Wadowice, Voivodeship Krakow, Poland, 
previously in Southern Galicia) with captions in manuscript, in German. The album was bound by Robert Jahoda. In an oblong folio bound in a brown morocco binder. On the cover is included a large metal coat of arms of the Archduke of Austria, Rainer, in the center, as well as various metal corner ornaments with a metal clasp. Krakow (Poland). Rainer, Archduke of Austria, 1827-1913.

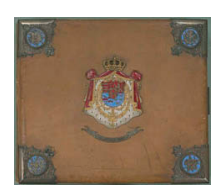

Slav. Reserve (Photo) 00-2385. SLV 018 [Cover title]; Hof Izdebnik von der Ostseite; Kirche und Schule in Izdebnik; Hof Jastrzebia; Hof Skawinki; Bahnstation Stronie; Hof Zakrzow; Forsthaus Lanckoron; Kirche und Schule in Sulkowice; Hof Harbutowice von der Ostseite; Stadt Lanckoron in der Ferne; Forsthaus Goscibia, Sudseite; Forsthaus in Budzow, Westseite; Kloster Kalwarya; "Die ehrfurchtsvoll und teu ergebenen Beamten und Diener der Herrschafft Lanckoron-Izdebnik."

\section{[Album of photographs of Russian countryside in Vyborg Region, north of St. Petersburg].}

[S.l.: s.n., 1910?] 72 mounted photos, $38.5 \times 19.5 \mathrm{~cm}$.

Album of 72 photographs depicting Russian countryside, family portraits, etc. Initials "S B" with a picture of a deer in between embossed on verso of back cover. Art Nouveau binding. Purchased from Alex Rabinovich, New York, 2000. Vyborgskii raion (Russia). Photograph albums-Russia-Specimens. Bookbinding-Specimens.

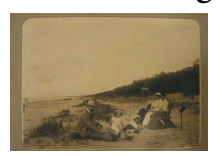

Slav. Reserve (Photo) 00-3335 SLV 019 1. a) Underpass of bridge, b) Child sitting in front of a house; c) View on a bridge; 2. a) Ladies walking around a stream, b) View close to forest, c) Near the stream; 3. a) Four women on and near large rock, b) People resting on a beach, c) Four women; 4. a) People are driving two boats, b) Entrance to house, c) Children on the porch of a house; 5 a) A group ready for a buggy ride, b) Summer house in the forest, c) Children standing on a fence and a woman nearby; 6. a) Path through the woods, b) Crossing the bridge, c) Riding in a buggy; 7. a) Family in a doorway of a house, b) Path of the trees, c) Three children and a woman on a bench; 8. a) Pathway, b) Group of people in a summer house, c) Fallen tree; 9. a) Child on top of the bench, b) Field with giant tree, c) Moon way; 10. a) Five people in the woods, b) Three dressed up people, c) A couple; 11. a) A group ready for a tennis match, b) Family picture on the stairs of a house, c) Family picture in the woods; 12. a) Ready for a horse ride, b) Herd of sheep, c) Boats; 13. a) People close to pond, b) Stream with rocks in the wood, c) A house with a flag; 14. a) Large trees, b) A dock, c) Houses and grove on the water; 15. a) Sun reflection in the water, b) Fences, c) Huge tree; 16. a) Large ladder against a house, b) Trees on water's edge, c) A big house; 17. a) Wooden bridge, b) Waterway, c) Women near bridge; 18. a) Walk in 
the woods, b) Resting on the bench, c) Resting in the woods; 19. a) A view, b) View through the trees, c) Forest and river; 20. a) A group of people, b) Duplicate of a, c) Sanatorium "Pearl"; 21. a) A house, b) A group of people, c) A house; 22. a) Two women in the field, b) Lady with a parasol, c) Sunlight; 23. a) Road to a house, b) View of a waterway, c) Beach with a cliff; 24. a) Rocks, b) Rocky bridge over water, c) Forest

\section{Aleksandro-Nevskaia lavra $v$ S. Peterburge. Fotograficheskii Al'bom.}

[S.l.: s. n.], 1874. 14 photographs, $35 \times 49.5 \mathrm{~cm}$. Cover title. Titled in gold on velvet binding, decorated with gilt metal frame and ornament. Metropolitan New York Library Council, 2008. Slavic Recon Project. Aleksandro-Nevskaia lavra (Saint Petersburg, Russia). Monasteries- Russia (Federation) - Saint Petersburg.

Slav. Reserve (Photo) (Aleksandro-Nevskaia lavra v S. Peterburg) SLV 020 [Title cover]; [Plaza in front of the Monastery]; [Trinity Cathedral]; [Trinity Cathedral, and view of the necropolis]; [Chapel within Monastery]; [Cemetery Chapel]; [Reliquary of Alexander Nevskii]; [Mitre with ermine]; [Mitres with panagias and cross]; [Funeral monument of Mikhail Vorontsov]; [Tombstone of an angel]; [Mausoleum of Andrei N. Murav'ev]; [Tomb of Speranskii]; [View of Monastery (Lavra) from the far bank of the Neva]; [Chapel outside the Gates]

Barshchevskii, I. F. (Ivan Federovich), 1851- 1948.

Al'bom fotograficheskikh snimkov s tserkovnoi arkhitektury $v$ Iaroslavskoi gubernii is Kniazhikh teremov v Rostove (Iaroslavskoi gubernii.) Rostov: Fot. I. F. Barshchevskii, 1884? 33 mounted photographs, $31 \times 25.5 \mathrm{~cm}$. Title supplied by cataloger. Issued in portfolio.

With bookplate of Grand Duke Vladimir Aleksandrovich. 33 photographs showing exteriors and interiors of religious and imperial landmarks in Iaroslav province.

Some photos show buildings before, during, and after repairs and reconstruction. Some photos (plates 4, 14, 15, 23, 25) show people (peasants, workers) in foreground. Barshchevskii was the photographer of the Imperial Archaeological Society. Metropolitan New York Library Council, 2008. Slavic Recon Project.

Church architecture-Conservation and restoration. Church architecture Russia (Federation).

Slav. Reserve (Photo) (Albom fotograficheskikh snimkov s tserkovnoi arkhitektury v Iaroslavskoi gubernii) SLV 021 Gor. Iaroslavl'. Tserkov' Il'i Proroka s Iugo-Zapadnoi storony; Gor. Iaroslavl'. Tserkov' Il'i Proroka s Severo-Vostochnoi storony; Gor. Iaroslavl'. Obshchii vid tser. Ioanna Zlatousta v Korovnikakh s Vostochnoi storony; Gor. Iaroslavl'. Tserkov' Ioanna Zlatousta v Korovnikakh s SeveroZapadnoi storony; Gor. Iaroslavl'. Tserkov' Ioanna Predtechi v Tolchkove 
s Severo-Zapadnoi storony; Gor. Iaroslavl'. Iuzhnaia storona tserkvi Ioanna Predtechi v Tolchkove; Gor. Iaroslavl'. Vkhod teploi tserkvi u Nikoly Mokrinskogo s Iugo-Zapadnoi storony; Gor. Iaroslavl'. Tsarskoe mesto v tserkvi Nikoly Mokrinskogo na pravoi storone; Gor. Iaroslavl'. Tsarskoe mesto v tserkvi Nikoly Mokrinskogo na levoi storone; Gor. Iaroslavl'. Tserkov’ Petra i Pavla chto na Volgskom beregu s Severo-Zapadnoi storony; Gor. Iaroslavl'. Tserkov' Rozhdestva Khristova s Kolokol'nei s Severo-Zapadnoi storony; Tser. Sv. Sergiia s iuzhnoi storony v Borisogleb. monast. bliz Rostova Iar.; Zapadnaia dver' tser. Sreteniia v Borisoglebskom monastyre bliz Rostova Iar.; Detal' sviatykh vorot v Borisoglebskom monastyre bliz Rostova Iar.; Tser. Ioanna Bogoslova na reke Ishne, bliz Rostova Iarosl. gub. postr. 1685 g. s iuzhnoi storony; Detal' dereviannykh reznykh tsarskikh vrat v tser. Ioanna Bogoslova na r. Ishne bliz Rostova Iar. gub.; Detal' dereviannykh reznykh tsarskikh vrat v tser. Ioanna Bogoslova na r. Ishne bliz Rostova Iar. gub.; Detal' dereviannykh reznykh tsarskikh vrat v tser. Ioanna Bogoslova na r. Ishne bliz Rostova Iar. gub.; Dereviannye reznye tsarskiia vrata raboty 1562 g. v tser. Ioanna Bogoslova na r. Ishne bliz Rostova Iar. gub.; Vkhodnye dveri $\mathrm{v}$ tser. Ioanna Bogoslova na reke Ishne, bliz Rostova; Vodosviatnaia Chasha v Avraamievskom monastyre bliz Rostova; Fasad Kniazhikh Teremov do vozobnovleniia; Fasad Kniazhikh Teremov, posle vozobnovleniia ikh - v 1884 godu; Fasad Kniazhikh Teremov do vozobnovleniia ikh.; Fasad Ioninskoi palaty, posle vozobnovleniia; Iuzhnaia stena Teremov (uchilishche Sv. Dimitriia) (po vozobnovlenii) v Rostove Iar. gub; Iuzhnaia stena Teremov (uchilishcha Sv. Dimitriia) (do vozobnovleniia) v Rostove Iar. g.; Vnutrennii vid terema "otdatochnago," kotoryi nerazryvno smezhen s bashnei, znachushcheisia v proekte pod litf N.; [Interior view of chamber]; Otdatochnaia Palata posle vozobnovleniia v 1883 g.; [Otdatochnaia Palata posle vozobnovleniia v 1883 g.]; [Interior view of chamber before renewal]; [Interior view of chamber after renewal]

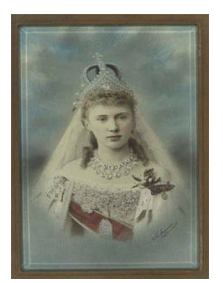

Bergamasco, Charles, 1830-1896. [Photograph portrait of a member of Russian royal family]. [S. 1.: s. n., 188-] 1 photograph (colored), $45 \times 31 \mathrm{~cm}$. In leather case under glass. Fekula Collection. Romanov, House of - Portraits. Slav. Reserve (Photo) 98-5231 SLV 023 [A portrait of Russian noble woman]

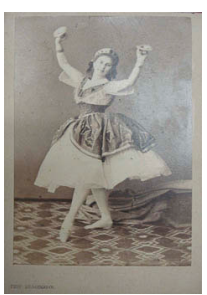

Bergamasco, Charles, 1830-1896. [Russian ballet dancers in photographs taken by C. Bergamasco]. [St. Petersburg: s.n., 1870-1889?] 22 photographs, $10 \times 6 \mathrm{~cm}$. Original photographic prints (carte de visites). One photograph taken by M. Konarskii. Gift of Alex Rabinovich, 2007. BalletCostume. Ballet dancers.

Konarskii, M. Slav. Reserve (Photo) 07-3509 SLV 024 Liadova (?); Liadova (?); Madaeva (?) and Usenshinski (?); Radina (?); Pririkhunova (?); Unknown; Radina (?); Grantsova (?); Unknown; Unknown; Radina, Fabr, Prikhunova, Zaitseva, 
Simskaia, Lanch (?), Granken (?); Dark (?); Unknown; Murav'eva (?); Kosheva and Ivanov; Petipa; Madaeva and Unknown (?); E. P. Sokol (?); Nemerer (?); Liadova (?); Sobeshchanskaia (?); Unknown; Radina (?)

Bokov, V. E. Posieshchenie vysochaishimi osobami Zlatoustovskikh kazennykh gornykh zavodov.

Ufa: Gubernskaia tipografiia, 1904. ii, 84 p., 19 leaves of plates, $24.5 \times 17.5 \mathrm{~cm}$. Binding bears embossed initial ' $\mathrm{V}$ ' below a crown (presumably initial of Grand Duke Vladimir Aleksandrovich). "Perl. Jun 18, 1931.” Metropolitan New York Library Council, 2008.

Slavic Recon Project. Minerals -Russia (Federation) -Zlatoustovskii uiezd. Zlatoustovskii uiezd (Russia) - History. Zlatoustovskii uiezd (Russia).

Slav. Reserve (Photo) (Bokov. Posieshchenie) SLV 095 [Alexander II]; [View of a mine factory]; [A wooden house]; [General view of fiscal mine]; [A tower]; [Monument to Alexander I]; [Alexander Nikolaevich in 1837]; [Alexander Nikolaevich at a peasant's hut, during his trip around Russia]; [An exhibition room]; [Imperial family at a ruined pavilion]; [A royal person on the middle of cliff]; [Two unidentified men]; [Duke Maksimilian of Leikhtenoergsk]; [An officer]; [A decorated pavilion]; [An unidentified military young man]; [A display room]; [A statue of Alexander II]; [General view of the city of Zlatoustovsk]

Chizhov, A. Mtskhetskii Khram: istoricheskoe $i$ arkhitekturnoe opisanie. Tiflis: Tipografiia Melikova i Ko., 1871.18 p., 4 leaves of plates, $30 \times 24.5 \mathrm{~cm}$. With bookplate of Tsar Alexander II and stamp of Tsarskoe Selo library. Fekula Collection, no. 6817.

Church buildings-Georgia (Republic)-Mtskheta. Architecture-Georgia (Republic)-Mtskheta. Mcxeta (Georgia)-Buildings. Mcxeta (Georgia)Antiquities.

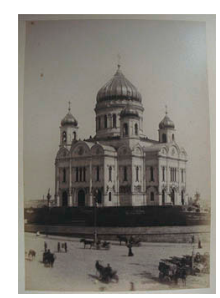

Slav. Reserve (Photo) 01-3704 SLV 026 1. Mtskhetskii Sobornyi Khram; 2. Tserkov's kupoloobraznoi krovlei raspolozhenaia naprotiv Mtskhetskogo Sobora; 3. Riznitsa Mtskhetskogo Sobora, sokhranivshiia neskol'ko predmetov upominaetsia v gramotakh tsarei; 4. Pervaia tserkov' sooruzhennaia tsarem Marianom $\mathrm{v}$ predele kotoroi on polozhil Sviatyiu propovednitsy Niny

Daziaro, J. Souvenir de Moscou.

Moscow: J. Daziaro, ca. [1875-1890?] 1 album (12 photographs), $18 \times 25 \mathrm{~cm}$. Cover title. Photographs mounted on card and with a Russian, French, and English caption on verso. Original publisher's blue cloth with gilt and black decorations and embossed lettering on upper cover. Purchased from Bernard J Shapero Rare Books, 2007. 
Moscow (Russia)-Pictorial works. Photography-Russia-19th century. Firma Dzhuzeppe Datsiaro.

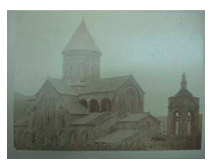

Slav. Reserve (Photo) 07-6531 SLV 027 1. Moskva, Kreml' ot Moskvaretskago mosta; 2. Moskva, vid Krasnoi ploshchadi; 3. Moskva, Tsar' pushka v Kremle; 4. Moskva, dom boiar Romanovykh; 5. Moskva, Lubianskaia ploshchad'; 6. Moskva, Novo-Devichii monastyr'; 7. Moskva, Uspenskii sobor v Kremle; 8. Moskva, Tsar' kolokol v Kremle; 9. Moskva, vid Khrama Khrista Spasitelia; 10. Moskva, Tserkov' Vasiliia Blazhennago; 11. Moskva, Imperatorskii Istoricheskii muzei; 12. Moskva, vid Iverskikh vorot

Daziaro, J. Moscou. [Moscow: J. Daziaro, 1880?] 1 album (27 photographs): col. ill.; $30 \times 40 \mathrm{~cm}$. Title page hand lettered. Handcolored photographs mounted on cards. Bound in burgundy morocco, gilt.

Captions to some illustrations in Russian and/or French. Purchased from Bernard J Shapero Rare Books, 2007. Moscow (Russia). PhotographyRussia-19th century. Firma Dzhuzeppe Datsiaro.

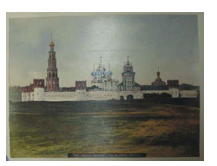

Slav. Reserve (Photo) 07-6534 SLV 028 1. Vid Kremlia s kamennym mostom; 2. Palais Impérial au Kremlin; 3. [Spasskaia Tower in Kremlin]; 4. [Spasskaia Tower and the Senate in Kremlin]; 5. [Inside the Kremlin: Spasskaia Tower and the Senate]; 6. [Imperial Palace of Kremlin]; 7. Cathédrale de l'Assomption au Kremlin; 8. [Andreevskii Hall]; 9. [Oruzheinaia Palata]; 10. Ivan Veliky au Kremlin; 11. [Dormition Cathedral]; 12. [Iconostasis, Dormition Cathedral]; 13. [Cathedral of the Annunciation]; 14. Cathedrale de St. Michel Archange; 15. Vid Devich'iago Monastyria; 16. [A square in Moscow]; 17. [A house in Moscow]; 18. [Cathedral of Christ the Savior]; 19. [Unidentified church]; 20. [Unidentified church]; 21. Tserkov' Pokrova na Filiakh; 22. Tserkov' Rozhdestva Bogoroditsy; 23. [Unidentified building]; 24. [Unidentified gates]; 25. Tserkov' Vasiliia Blazhennago; 26. Eglise Wassili Blagenny; 27. Arc de Triomphe

Dalmatov, K. (Konstantin) [Al'bom obraztsov kharakternago starinnago narodnago uzornago shit'ia, tkani i russkikh kruzhev].

St. Petersburg: Khromolitografiia i tipografiia R. Khorn, 1888. 16 mounted photographs, $47 \times 35 \mathrm{~cm}$. Binder's title. Marked "11/23/30" and stamped "Mar. 5, 1934."

Includes various notes regarding K. D. Dalmatov from: Pravitel'stvennyi vestnik; Imperatorskoe Russkoe geograficheskoe obshchestvo; Imperatorskii Rossiiskii Istoricheskii Muzei; Moskovskiia Vedomosti.

"Perlstein Jun. 18, 1931." Metropolitan New York Library Council, 2008. Slavic Recon Project. Embroidery-Russia. 


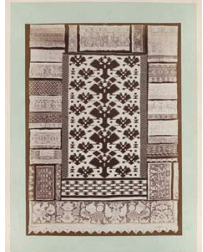

Slav. Reserve (Photo) (Dolmatov. Albom obraztsov kharakternago...) SLV 030 [Examples of characteristic old popular design embroidery, textiles, and Russian lace....]

Dalmatov, K. (Konstantin) [Obraztsy starinnago narodnago uzornago shit'ia i kruzhev]. [St. Petersburg: s.n., 1883-1894]. 7 v. of 350 mounted photographs, $24.5 \times 18 \mathrm{~cm}$. Title from the booklet. Vol. 1, the first pl. is a facism.; t-p. reads: Narodnoe uzornoe shit'e, tkan'e i vypletenie, sobrannye K. Dalmatovym.

"Jan. 16, 1941." Each volume is accompained by a descriptive booklet, in ms., from which dates and captions have been supplied; only booklets 1-3 are dated. Metropolitan New York Library Council, 2008. Slavic Recon Project.

Embroidery-Russia. Narodnoe uzornoe shit'e, tkan'e i vypletenie, sobrannye K. Dalmatovym.

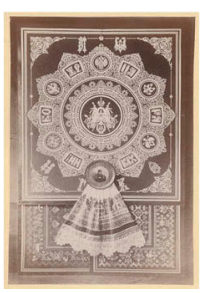

Slav. Reserve (Photo) (Dolmatov. Obraztsy...) SLV 031 Vol. 1 (1893): Narodnoe uzornoe shit'ie i kruzheva iz kollektsii K. Dalmatova. [Cover title] Polotentse tsvetnymi shelkami $\mathrm{v}$ tambure s izobrazheniem ptitsy Sireny, Starinnaia rabota Krest'ianok Novgorodskoi gub.; Dva starinnykh kontsa ot polotenets. Rabota po kiseiu tsvetnymi shelkami v tambure, krest'ianok Novgorodskoi gub.; Starinnoe polotentse ispolnennoe po kholstu tsvetnymi shelkami $\mathrm{v}$ glad i obmetku. Rabota i uzor krest'ianok Novgorodsk. gub.; Starinnoe polotentse ispolnennoe po kholstu tsvetnymi sherstiami. Rabota i uzor krest'ianok Moskovsk. gub.; Chetyre starinnykh polotentsa iz koikh dva verkhnikh ispolneny krest'iankami Iaroslavskoi gub. sela Kurba v vystrochku tsvetn. shelkami. Levoe nizhnee Novgorodsk. gub. a pravoe Moskovskoi gubernii; Kruzheva rybnoi Slobody Kazanskoi gub. a starinnoe polotentse $\mathrm{v}$ seredine Raboty i uzora Moskovskikh krest'ianok. Ispolneno po kholstu tsvetnami shelkami i zolotom v glade; Polotentse s dvukh raznykh kontsakh. Kargopol'skoi uezda Olonetskoi gub. Izobrazhaet lev zver' i ptitsu dvuglavago orla. Ispolneno po kholstu tsvetnymi sherstiami; Starinnoe polotentse Novgorodskoi gub. Ispolnennoe v vystrochku po polotnu krasnymi kumachevymi nitkami; Starinnoe polotentse beloi vystrochki po polotnu. Raboty i uzory krest'ianok Iaroslavsk. gub.; Starinnyia polotentsa pervoe tsvetnoi a vtoroe beloi vystrochki. Rabota i uzor Novgorod. gub.; Kontsy ot polotenets beloi vystrochki krest'ianok Iaroslavskoi gub.; Kontsy ot polotenets beloi vystrochki Iaroslavsk. gub.; Nebol'shaia nastol'naia skatert' raboty $\mathrm{v}$ glykhoi kreste po zabrannomu kholstu i polotentse $\mathrm{v}$ vystrochku krasnoi kumachevoi nitkoiu Rabota i uzor Novgorodskoi gub.; Kontsy ot polotenets beloi s krasnoi vystrochki Tverskoi gubernii; Starinnyia polosy vyshivok ot derevenskikh prostynei Novgorodsk. gub. Ispolneny po kholstu $\mathrm{v}$ glukhoi kreste prostoi kraskoi Kumachevoi nitkoiu i raznymi tsvetnymi shelkami; Tri polosy ot prostynei ispolneny v beluiu vystr. po kholstu Iz Belozerskago uezda Novgorodsk. gub.; 
Polotentse i dve polosy beloi vystrochki ot prostynei Verkhiiaia izobrazhaet okhotu i nizhniiaia skazku pro Ivana Tsarevicha. Rabota i uzor krest'ianok Kostromskoi gub.; Starinnyia raboty po kisee i polosy v vystrochku po vyrezu konets ot polotentsa, polnoe polotentse i polosa ot prostyni. Rabota Arkhangelsk. gubernii; Tri polotentsa i polosa beloi vystrochki po vyrezu Raboty i uzory krest'ianok Pudozhskago uezda Olonetskoi gubern.; Dve starinnyia kiseinyia zanoveski iz okna Raboty v tambure i mel'chaishuiu zateilivuiu strochku krest'ianok Torzhkovsk. uezda Tverskoi gub.; Samyia starinnyia kruzheva iz goroda Galicha ispolneny na kokoshnikakh tsvetnymi shelkami i zolotymi nitkami; Samyia starinnyia kruzheva Vologodskoi raboty nazyvaemyia Vologodskyia okna ili stekla. Dlinnaia polosa ispolnenyia iz tsvetnago shelka; Starinnyia kruzhevnyia polotentsa i polosy ot prostynei raboty i uzora Belozerskago uezda Novgorod. gub.; Starinnyia kruzhevnyia polotentsa i polosy ot prostynei raboty i uzora Belozerskago uezda Novgorod. gub.; Kontsy ot polotenets krasnoi vyshivki v glukhoi krest. Raboty i uzora krest'ianok Pskovskoi i Novgorodsk. gub.; Ser'gi kakoshchnik fata, kholodnik i parchovyi sarafan. Starinyi kharakternyi kostium devushki iz goroda Torzhka Tverskoi gub.; Starinnyia malorossiiskie rushniki t. e. polotentsa raboty i uzora Khar'kovskoi, Poltavskoi i Ekaterinoslavskoi gubernii; Starinnyia malorossiiskiia vyshivki ot podolov zhenskikh rubashek. Raboty i uzora Kievskoi gub.; Vse chasti polnago narodnago kostiuma Cheremisski Kazakhskoi gubernii. Uzory vyshity po grebomu kholstu chernym i telko tuntsovym shelkami; Devitsa v polnom velikorusskom kostiume Torzhskovskago starinnago nariada; Devitsa v nastoiashchem Malorossiiskom kostiume. Nariada Chernigovskoi gubernii Vol. 2 (1894): Obraztsy russkago starinnago kruzheva Tverskoi Novgorodskoi i Kostromskii guberni skopirovannye K. Dalmatovym i vyrezannye iz bumagi iz sobranii raznykh kollektsionerov. Tablitsa N. 16 sostavlena samim Dalmatovym i v 1887 godu podnesena Ikh Imperatorskim Velichestvam;

Kruzhevnyia vstavki ot polotenets Tverskoi gubernii; Kruzheva ot polotenets i prostynei Novgorodskoi gubernii Belozerskago uezda; Kruzheva ot polotenets i prostynei Novgorodskoi gubernii Belozerskago uezda; Kruzheva ot polotenets i prostynei Novgorodskoi gubernii Belozerskago uezda; Kruzheva ot polotenets i prostynei Novgorodskoi gubernii Belozerskago uezda; Kruzheva ot polotenets i prostynei Novgorodskoi gubernii Belozerskago uezda; Kruzheva ot polotenets i prostynei Novgorodskoi gubernii Belozerskago uezda; Kruzheva iz tonchaishikh shelkovykh nitok i vybrannyia v vidy strochek po polotny Vologodskii gubernii; Kruzheva Rostovskago uezda Iaroslavskoi gub.; Kruzheva iz Galitskago uezda Kostromskoi gubernii; Kruzheva Kazanskoi gub. Rybnoi Slobody;

Vol. 3 (1894): Vyshivki iz Pskovskoi i Novgorodskoi gubernii. Tablitsa N. 2 izobrazhaet starinnuiu obedennuiu skatert' i N. 9 zanovesku ot derevenskago okna. Vse ostal'nye NN. - polosy vyshivok ot starinnykh prostynei; Polosy 
shirokikh uzornykh vystrochek ot prostynei iz Kostromskoi gubernii; Polosy shirokikh vystrochek ot prostynei iz Pskovskoi i Novgorodskoi gubernii, N. 23 i 24 izobrazhaiut skazku ob Ivane Tsareviche; Iz sela Kurba Iaroslavskoi gubernii Ispolneny starinnymi tsvetnymi shelkami nastilnago ikh v glad'; Kruzheva Novgorodskoi gubernii; Zhenskaia rubashka Olonetskoi gubernii Pudozhskago uezda; Naplechinaia vyshivka ot rubashek toi zhe mestnosti; Kontsy ot polotentsa Olonetskoi gubernii Pudozhskago uezda vyshityia tsvetnymi sherstiami i belyia vyshivki dlia prostyn' i polotentsa iz Kargopol'skago uezda; Vyshivki chukhonok Peterburgskoi guber. bliz Strel'n; Vyshivki tsvetnymi shelkami mordovoi Penzovskii gubernii; Shit'e v tambure shelkami po tsvetnoi materii tatarok Nizhegorodskoi gub.; Vyshivki tsvetnymi shelkami votiachek Viatskoi gubernii;

Vol. 4: Kontsy ot polotenets raboty v glukhoi krest. Uzory iz Pskovskoi, Novgorodskoi i Vladimirskoi gubernii; Kontsy ot polotenets i prostynei v vystrochku. Tversk. gub.; Iz Belozerskago uezda Novgorodskoi gub.; Iz Iaroslavskoi gub.; Iz Kostromskoi gubernii; Iz Sela Kurba Iaroslavskoi gubernii; Iz Olonetskoi gub.; Iz Staritskai uezda Tverskoi gubernii; Polosy belykh vystrochek ot prostynei iz Kostromskoi gubernii;

Vol. 5: Vyshivki ot polotenets i prostynei raboty i uzora krest'ianok S Peterburgskoi gubernii Peterburgskago [?]davskago i Tsarskosel'skago uezdov; Vyshivki ot polotenets i prostynei raboty i uzora krest'ianok Iamburgskago uezda Peterb. gubernii; Vyshivki ot polotenets i prostynei raznykh uezdov Novgorodskoi gubernii; Vyshivki ot polotenets i prostynei Zubtsovskago i Novotorzhskago uezdov Tverskoi gubernii; Vyshivki ot polotenets i oplechii zhenskikh rubashek iz Staritskago i Zubtsovskago uezdov Tverskoi gubernii Ispolneny v vystrochku po polotnu i obvivku raznotsvetnymi sherstiami;

Vyshivki raznotsvetnymi shelkami v glukhoi krest Krest'ianok Tverskoi gubernii raznykh uezdov Uzory eti sostavliaiut peredniuiu chast' ukrasheniia mestnykh golovnykh uborov

Vol. 6: Uzornye kontsy ot polotenets i prostynei Kasimovskago uezda Riazanskoi gubernii vytkannye na ruchnykh stankakh iz raznotsvetnoi belevoi i shelkovoi priazhi; Tkanye kontsy ot polotenets Iamburgskago uezda Peterburgskoi gubernii; Tkanye kontsy ot polotenets i podolov zhenskikh rubashek raboty i uzora Krest'ianok Gzhatskago uezda Smolenskoi guber;; Tkanye kontsy ot polotenets Nizhegorodskoi i drugikh gubern.; Starinnyia nagrudnyia vyshivki tsvetnymi shelkami s zolotom i serebrom ot zhenskikh prazdnichnykh rubashek Krest'ianok Shatskai i drugikh uezdov Tambovskoi gubernii; Starinnyia polosy shirokikh belykh vystrochek ot prostynei iz Belozerskago uezda Novgorodskoi gubernii; Starinnye parchovye platki i golovnye ubory Krest'ianok Kostromskoi gubernii;

Vol. 7: Devushka v prazdnichnom nariade Cheremissok Viatskoi gubernii; Golovnoi ubor i fartukh cheremisskago nariada toi zhe gubernii; Naplechnyi vyshityi platok i v seredine ego zhenskii golovnoi ubor nariada cheremisski pomeshchennyi na tabl. Ioi; Zhenskaia cheremisskaia rubashka nariada 
oboznachenogo na tabl. No 1-oi.; Ugol nasheinogo platka polotentse i vetkhiia vyshivki ot zhenskikh golovnykh uborov Viatskoi gubern.; Nagrudnyia vyshivki ot zhenskikh cheremisskikh rubashek iz kazanskoi gubernii; Nagrudnaia vyshivka ot rubashek i vyshityi rukav Mordovke Ufimskoi gub.; Dolevyia vyshevki ot rukavov zhenskikh rubashek toi zhe gubernii; Vyshivki ot oplechii i obshlagov rukavov detskikh mordovskikh rubashek; Melkie i krupnye uzory prikryvaiushchie shvy zhenskikh chuvashskikh rubashek raznykh gubernii; Nagrudnyia vyshivki $\mathrm{v}$ vide zvezdy ot zhenskikh chuvashskikh rubashek Kazanskoi i drugikh gubernii;

Vyshivki ot nagrudnykh prorekh muzhskikh i zhenskikh rubashek raznykh gubernii. Uzory eti vse ispolneny raznotsvetnymi shelkami i sherst'iu $\mathrm{v}$ mel'chaishuiu glad

Dalmatov, K. (Konstantin) Pervaia vystavka rossiiskago narodnago uzornago shit'ia ustroennaia $v$ 1883-1884 g. K. Dalmatovym v S. Petersburge St. Peterburg: [s. n., 1884?] 19 mounted photographs, $33 \times 48 \mathrm{~cm}$.

Cover title. "Perl. Jun 18, 1931." Metropolitan New York Library Council, 2008. Slavic Recon Project. Embroidery-Russia. Embroidery-RussiaExhibitions.

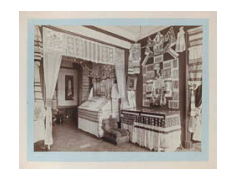

Slav. Reserve (Photo) (Dolmatov. Pervaia vystavka...) SLV 032 [Cover title]; [Assortment of different lace samples]; [Different lace displayed in geometrical design]; [A booth of different Russian embroidery designs]; [Embroidery curtain "Valences" and small examples of lace]; [Large room with displays of embroidery towels, tableclothes, pillow cases, costumes, dolls and napkins, etc.]; [Display of embroidery designs]; [A booth for Russian embroidery in geometrical design leaves, flowers, of textiles]; [A booth of Russian lace with flowered design of animals and peoples]; [Linens and lace with small examples of pins and board embroidery from Central Asia]; [Pieces of embroidery with views of interiors and traditional costumes of Eastern Russia, different embroidery]; [General view of the exhibition, featuring tablecloths and dolls in different costumes,]; [A fireplace screen, pillow lace, tablecloths, a big doll, and embroidered bonnet]; [Drapes assembled with different samples of embroidery in numerous designs. Fireplace screen, a big doll, laboratory design table covers...]; [A bedspread towel, a picture of peasant girl in a frame, and curtains]; [A whole set of lace samples in different geometrical designs, skirts, peasant blouses, and towels]; [Different samples of lace, embroidery, tablecloths, and lace for borders]; [Different samples of lace, embroidery costumes, tablecloths, and lace for borders]; [Different samples of lace, tablecloths, and lace for borders]; [Different samples of embroidered tablecloths, and lace for borders]

Dalmatov, K. (Konstantin) Sbornik starinno-russkikh, slavianskikh bukv, zastavits $i$ kaemok dlia knigopechatnoi $i$ perepletnago, graviroval'nago $i$ vyshivnago diel. 
St. Petersburg, 1895. 35 mounted photographs: sepia; $33 \times 24 \mathrm{~cm}$. An album of old Russian and Slavonic letters, headpieces and decorative borders for bookprinters, bookbinders, engravers, and embroidery. Ms. note on verso of cover: "Bibl. Imper. Aleks. III" (Library of Alexander III). List of subscribers to the album, in ms., [2] p., bound at end.

Monograms-Specimens. Lettering-Specimens. Book ornamentationRussia. Cyrillic alphabet-Specimens.

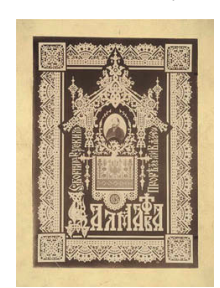

Slav. Reserve (Photo) 93-451 SLV 033 Sbornik starinnorusskikh, slavianskikh bukv, zastavits i kaemok dlia knigopechatnoi i perepletnago, graviroval'nago i vyshivnago diel/sostavlen K. D. Dalmatovym. No. 1 through No. 35.

Domik Petra Velikago $v$ Vologdie. St. Petersburg: Tipografiia V. Kirshbauma, 1887. 30 p., 14 mounted photographs, $30 \times 20.5 \mathrm{~cm}$.

On p. 1 of text: red stamp "Perl. Jun 18, 1931." With it is bound: Opisanie istoricheskago ugolka goroda Vologdy i muzeia pri nem.

"An album of 14 albumen prints approx. 14 x $22 \mathrm{~cm}$ glued on mounts with captions under the images, in the negative, stamped lower right: Fot. K. L. Baran'ev illustrating two booklets: 1) "Peter the Great's small house in Vologda, St. Petersburg, 1887, 30p. signed: An inhabitant of Vologda. 2) "Aion of and historic corner of the city of Vologda and its museum - 1885. 5 June.1887" St. Petersburg, Anonymous. The two booklets and the photographs are bound in a black leather binding, with silk moire and gilt decoration inside the covers. The photographs show the House of Peter the Great, a general view of the city, the Church of St. Theodore Stratilate, two different iconostases ("from the warm church", and "from the cold one"), several inside views showing vitrines, views of the square, garden cannon, and a monument appearing to be with the monogram of Peter the Great." (Pierre Apraxine) “Perl. Jun 18, 1931" Peter I, Emperor of Russia, 1672-1725. Museums - Russia - Vologda.

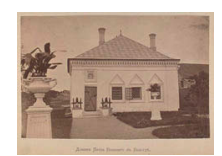

Slav. Reserve (Photo) (Domik) SLV 034 Domik Petra Velikago s chast'iu skvera; Domik Petra Velikago v Vologdie; Petrovskii skver; Obshchii vid Istoricheskago ugolka g. Vologdy; Tserkov' Sv. Feodora Stratilata; Ikonostas kholodnago Khrama; Ikonostas teplago Khrama; Kiot $s$ obrazom Spasa Nerukotvorennago; Vnutrennii vid bol'shoi komnaty, s portretom Petra Velikago; Ugol bol'shoi komnaty s kiotom, analoiami i vitrinami; [Entryway foyer];

Biust Petra Velikago v tsvietnikie; Starinnaia Vologodskaia pushka; Bilo 1706 g. s venzelem Petra I v lavrovom vienkie s koronoi

Eia Imperatorskago Vysochestva Velikoi Kniazhny Anastastii Nikolaevny Voenno-sanitarnyi poiezd no. 61. [1916?]. 14 leaves of plates (some col.), $37 \times 41 \mathrm{~cm}$. 
Ms. label of the Alexandrov Palace Library pasted on verso of hard cover: "Aleksandr. dv. muz. detsk. pol. Klassnaia starsh. kniazhn., \# 174". In a portfolio of blue buckram with the gilt crowned monogram of Anastasiia Nikolaevna, Grand Duchess, daughter of Nicholas II, Emperor of Russia, 1901-1918 Six original drawings of A.N. Tyshkevich.

Hospital trains - Russia. World War I, 1914-1918 - Hospitals.

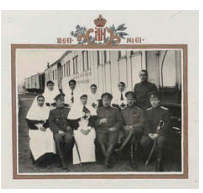

Slav. Reserve (Photo) 08-3073 SLV 035 [Binder's title]; Eia Imperatorskago Vysochestva Velikoi Kniazhny Anastastii Nikolaevny Voenno-sanitarnyi poiezd No. 61; [The first year of activation (1914) of Military Hospital Train (V.S.P.N. 61).]; [Map of railway]; [Crew members of VSP No. 61 and nurses]; [Interior view of VSP No.61]; [Surgery on the train]; [Crew members of the military train]; [IThree interior and exterior views of the train]; LegkoRanenye. Tiazhelo-Ranenye; Tseppelin nad Minskom; Nochnoe dizhurstvo: (u legko ranenykh.); Nochnoe dizhurstvo: (u tiazhelo ranenykh.); V.S.P. No.:61; Vydacha provizii dlia ranenykh.

Fasady zdaniia $i$ vnutrennii vid masterskikh tipografii vtorago otdieleniia sobstvennoi Ego Imperatorskago Velichestva kantseliarii.

St. Petersburg: Fotografiia Shenfel'da, 1876. 12 mounted photographs, $33 \times 45 \mathrm{~cm}$. Binder's title. "Perl. Jun 18, 1931."

Slavic Recon Project. Metropolitan New York Library Council, 2008. Buildings-Russia - Saint Petersburg. Factories- Russia - Saint Petersburg.

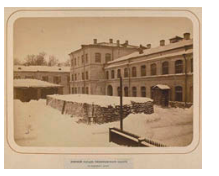

Slav. Reserve (Photo) (Fasady zdaniia...) SLV 036 [Binder's title]; Fasad tipografskago zdaniia po Inzhenernoi ulitsy; Fasad tipografskago zdaniia po Inzhenernoi ulitsy; Bokovoi fasad tipografskago zdaniia s peredniago dvora; Bokovoi fasad tipografskago zdaniia s peredniago dvora; Bokovoi fasad tipografskago zdaniia i kladovykh $\mathrm{s}$ zadniago dvora; Skoropechatnia v srednem etazhie; Skoropechatnia v nizhnem etazhie; Pechatnia na ruchnykh stankakh; 1-i zal nabornoi; 2-i zal nabornoi; Mekhanicheskaia i slesarnia; Stereotipnia.

Fotograficheskie snimki biustov Imperatorov Nikolaia I i Aleksandra II. 5 mounted Photographs, $35 \times 27 \mathrm{~cm}$. Contemporary morocco portfolio by P. O. Iablonskii of Saint Petersburg. Photographs of busts of Russian emperors sculptured by N. Laveretskii. There are two copies of this volume (one in its original portfolio), both of which have the same call number. Sculpture - Russia.

Nicholas I, Emperor of Russia, 1796-1855. Alexander II, Emperor of Russia, 1818-1881.

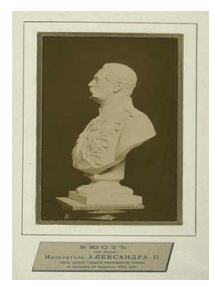

Slav. Reserve (Photo) (Fotograficheskie snimki biustov Imperatorov Nikolaia I i Aleksandra II). SLV 037 [Album title]; Biust (iz bronzy) Imperatora Aleksandra II pered zdaniem Gorodskoi Aleksandrovskoi bol'nitsy v pamiat' 19 Fevralia 1861 goda; Biust (iz bronzy) Imperatora Aleksandra II pered zdaniem Gorodskoi Aleksandrovskoi bol'nitsy v pamiat' 19 
Fevralia 1861 goda; Biust (iz mramora) Imperatora Nikolaia I v Gorodskoi Obukhovskoi bol'nitsie; Biust (iz mramora) Imperatora Nikolaia I v Gorodskoi Obukhovskoi bol'nitsie; Modeli Biustov Imperatorov Nikolaia I i Aleksandra II v masterskoi Professora N. Laveretskago.

Fotografii Khersonisskago khrama bliz Sevastopolia. [St. Petersburg: Tipo-lit.

Trofimova, 189-?] 4 mounted photographs, 47 x $36.5 \mathrm{~cm}$. Cover title. With autograph of academician N. Chagin on each print. "Perl.

Jun 18, 1931.” Metropolitan New York Library Council, 2008. Slavic Recon Project. Cathedrals - Ukraine - Kherson. Kherson (Ukraine).

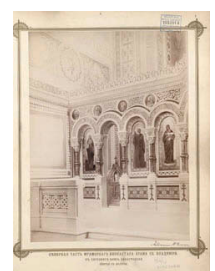

Slav. Reserve (Photo) (Fotografii Khersonisskago khrama bliz Sevastopolia) SLV 038 [Cover title]; Severnaia chast' mramornago ikonostasa Khrama Sv. Vladimira. $\mathrm{v}$ Khersonisie bliz Sevastopolia. sniataia $\mathrm{s}$ natury; Iuzhnaia storona vnutri Khrama Sv. Vladimira. v Khersonisie bliz Sevastopolia. sniataia s natury; Chast' sievernoi storony vnutrennosti Khrama Sv. Vladimira. v Khersonisie bliz Sevastopolia gdie sooruzhena kiota $\mathrm{v}$ pamiat' sobytiia 17 oktiabria 1888 g. sniataia s natury; Chast' vostochnoi storony vnutrennosti Khrama Sv. Vladimira. v Khersonisie bliz Sevastopolia. sniataia s natury.

Fotografii Novgorodskago Muzeia. [Novgorod: Tipo-litogr. Novgor. Gub. Pravleniia, 1893]. 22 mounted photographs, $32 \times 25.5 \mathrm{~cm}$. Inserted is one leaf end: Opisanie Fotografii Novgorodskago muzeia. Originally unbound, in portfolio of brocade and velvet, lined with purple satin. "Perl. Jun 18 1931." Slavic Recon Project.

Metropolitan New York Library Council, 2008. Museums - Russia (Federation) - Novgorod.

Slav. Reserve (Photo) (Fotografii Novgorodskago muzeia SLV 039

Naruzhnyi vid zdaniia muzeia i biblioteki; Listnitsa; Avan-zal; Avan-zal; Avan-zal; Avan-zal; Tserkovnyi zal; Tserkovnyi zal; Tserkovnyi zal; Tserkovnyi zal; Tserkovnyi zal; Tserkovnyi zal; Tserkovnyi zal; Tserkovnyi zal; Tserkovnyi zal; Tserkovnyi zal; Arsenal'nyi zal; Lestnitsa; Lestnitsa; Lestnitsa; Konferents-zal; Konferents-zal.

Fotografii s proekta Pravoslavnago Sobora dlia gor. Revelia. Tallinn, 1894.

8 mounted photographs; $33.5 \times 25 \mathrm{~cm}$. "Perl. Jun 18, 1931." Title from binding. Each photo signed: Akademik M. Preobrazhenskii. Cover title. Album is the project of the building of Aleksandro-Nevskii Cathedral. Church buildings - Estonia - Tallinn. Cathedrals-Estonia -Tallinn.

Slav. Reserve (Photo) (Fotografii s proekta Pravoslavnago sobora dlia gor. Revelia) SLV 040 [Cover title]; [General view of Aleksandro-Nevskii Cathedral]; Proekt Pravoslavnago 
Sobora dlia g. Revelia. Na 1500 cheloviek. Zapadnyi fasad; Proekt Pravoslavnago Sobora dlia g. Revelia. Na 1500 cheloviek. Vostochnyi fasad; Proekt Pravoslavnago Sobora dlia g. Revelia. Na 1500 cheloviek. Sievernyi fasad; Proekt Pravoslavnago Sobora dlia g. Revelia. Na 1500 cheloviek. Poperechnyi razriez; Proekt Pravoslavnago Sobora dlia g. Revelia. Na 1500 cheloviek. Prodol'nyi razriez; Plan po lin. dv.; Proekt Pravoslavnago Sobora dlia g. Revelia. Na 1500 cheloviek. Plan po lin. zhe. Plan po lin. ab.

Fotografii stiennoi zhivopisi $v$ bol'shom altarie Isaakievskago Kafedral'nago Sobora. [S.1.: s. n., 18-?] 7 mounted photographs, $47.5 \times 35.5 \mathrm{~cm}$. Binder's title. Inserted is one leaf containing hand-writing of plates, which is headed: Nazvanie fotografii s restavrirovannoi stiennoi zhivopisi v bol'shom altarie Isaakievskogo Kafedral'nago Sobora. "Perl. Jun 18, 1931.” Metropolitan New York Library Council, 2008.

Slavic Recon Project. Isaakievskii sobor (Saint Petersburg, Russia). Mural painting and decoration, Russian. Orthodox Eastern church buildings Russia (Federation) - Saint Petersburg.

Slav. Reserve (Photo) (Fotografii stiennoi zhivopisi v bolshom altarie Isaakievskago kafedralnago sobora) SLV 041 [Cover title]; Na plafonie potolka, kartina, "Vvedenie Pervorodnago Syna Bozheiia vo vselennuiu"; Na vostochnoi stienie, nad attikom v polukruzhii "Dukh Sviatoi okruzhennyi angelami"; Na iuzhnoi stienie attika "Omovenie noch"; $\mathrm{Na}$ vostochnoi stienie attika "Osiavanie Fomy"; Na sievernoi stienie attika "Iavlenie Gospoda Apostolam po voskresenii"/Vsie piat' kartin rektora Bruni; Nad Tsarskimi vratami "Nerukotvorennyi obraz", zhivopis' na miedu professora Nefa; Arkhitekturnyia ukrasheniia chasti svoda.

Kan, M.A. (Firm) Photograph of N. Gumilev. [S.l: s.n., s.d].

1 photoprint. An autographed photograph of N. Gumilev, Russian poet. $10.7 \times 6.3 \mathrm{~cm}$. Photograph has an inscription (front and back), certifying that the person on the picture is indeed the son of the civil servant Nikolai Stepanovich Gumilev, dated July 3, 1906 in Tsarskoe Selo, and signed, policeofficer V. Sakhorov. With stamp of $1^{\text {st }}$ police district of Tsarskoe Selo. Russian photographer in Tsarskoe Selo, Koniushennaia ul. From the collection of I. Martynov. Gumilev, N. (Nikolai), 1886-1921.

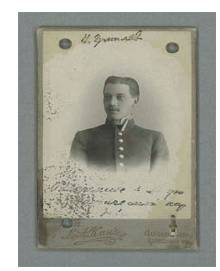

Slav. Reserve (Photo) 90-5900 SLV 042 N. Gumilev. Oznachennoe natsei fotograficheskoi kartochke [deisvitel'no] i oznachaet $\mathrm{m}[]$ syna statskago sovietnika Nikolaia Stepanovicha Gumileva. 1906, Iiulia 3 dn. g. Tsarskoe Selo. Pristav V [Sakharov].

G. Tikhvin i Tikhvinskaia sistema, 26-go iiunia 1887 g. [S l.: s. n., 1887] 15 mounted Photographs, $33 \times 42 \mathrm{~cm}$. Some plates stamped on verso: "Inzhener Buchatskii, G. Tikhvin, 30 AVG. 86." Markings: [in pencil, on 
plate 1] G. Tikhvin I. 10/19/33; stamped on back cover: "Mar. 29 1934." Title from original portfolio.

Handwritten letterpress. Slavic Recon Project. Metropolitan New York Library Council, 2008. Buchatskii, Longin Khrisanfovich, b.1843 Tikhvin (Russia). Inland water transportation- Russia -Tikhvin.

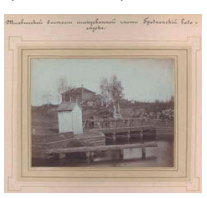

Slav. Reserve (Photo) (G. Tikhvin i Tikhvinskaia sistema) SLV 043 [Cover title]; Tikhvinskoi Sistemy vodorazdiel. Pamiatnik Imperatoru Petru 1-mu pri ozerie Krupino; Tikhvinskoi Sistemy shliuzovannoi chasti vodouderzhatel'naia plotina novago tipa; Tikhvinskoi Sistemy shliuzovannoi chasti novago tipa Smolenskii shliuz; Gorod Tikhvin i dom zanimaemyi Nachal'nikom Tikhvinskago Otdieleniia; Tikhvinskoi Sistemy r. Chagodoshcha-UstChagodotskaia pristan' i parom pri ust'ie r. Mologi; Usad'ba Grafa MusinaPushkina i parom na rieke Mologie na 172 verst; Tikhvinskoi Sistemy. Detal' ponurnoi chasti novoi Khersonskoi plotiny. Postroennoi Inzhenerom Buchatskim v 1885 godu na osnovanii vyrabotannago v s'iezdakh Inzhenerov Vytegorskago Okruga novago tipa; Tikhvinskoi Sistemy Mologskaia pristan' na rieke Mologie; Tikhvinskoi Sistemy r. Sias’ Kurgany pri derevnie Gorodishche; Tikhvinskoi Sistemy rieka Sias' Rozhestvenskii porog; Tikhvinskoi Sistemy shliuzovannoi chasti Grodnenskii vodospusk; Tikhvinskoi Sistemy shliuzovannoi chasti vodouderzhatel'naia plotina starago tipa; Tikhvinskoi Sistemy rieka Sias' usad'ba g. Lemana; Tikhvinskoi Sistemy rieka Tikhvinka i gorodskaia pristan'; Tikhvinskoi Sistemy rieka Sias' i derevnia Stolbovo.

Gautier, Théophile, 1811-1872. Trésors d'art de la Russie ancienne et moderne. [Treasures of ancient and modern Russian art]. Paris: Gide, libraireéditeur 1859-[1861]. 5 v. (60 illustrations). $73 \times 52 \mathrm{~cm} .41$ albumen silver prints by P. A. Richebourg; three parts bounds in 1 volume (incomplete copy).

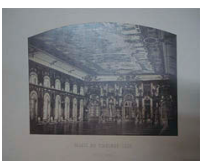
3-MAM+++ (Gautier, T. Tresors d'art de la Russe ancienne et moderne) MFZ+++(Richebourg) 16-4020 Part 1. Palais impérial de Tsarskoé-Sélo; 1. Façade sur la cour d'honneur; 2. Iconostase de la chapelle; 3 . Tribune impériale de la chapelle; 4 . Chambre a coucher de l'Empereur Alexandre Ier; 5. Salon de l'appartement d'Alexandre Ier; 6. Yantarnaïa zala; 7. Galerie de tableaux; 8. Vue en enfilade des appartements; 9. Grande salle; 10. Salle de potiches; 11. Antikamera pervaïa; 12. Arabeskovaïa zala; 13. Lionskaïa zala; 14. La veuve du marin; 15. Salon chinois; Part 2. Arsenal de Tsaraskoé-sélo; 1. Vue extérieure; 2. Salle des chevaliers; 3. Chambre turque; 4. Groupe de chevaliers; 5. Armes et armures; 6. Armes et armures; 7. Armes et armures; 8. Armes et armures; 9. Armes et armures; 10. Armes et armures; 11. Armes et armures; 12. Armes de chasse; 13. Armes a feu; 14. Dague florentine; Part 3. Saint- Isaac; 1. Saint Isaac de Dalmate. Mosaïque; 2. Vue générale. Côté sud; 3. Portique et fronton. Côté ouest; 4. Portique et vue de l'Amirauté; 5. Porte de bronze; 6. Grande iconostase; 7. Peinture de l'attique; 8. Paroi latérale de la Grande 
Iconostase; 9. Saint Jean a Pathmos. Pendentif; 10. Grande coupole; 11. Plafond de l'iconostase; 12. Concile d'apôtres. Tympan.

Geiman, S. V. (Sergei Veniaminovich) Tipy russkikh kolonistov $v$ S. Sh. A.: Sektanty, fermera, rybaki, uglekopy, i dr.; al'bom fotograficheskikh snimkov. [S.l: s.n., 191-?]. $20 \times 36 \mathrm{~cm}$. 1 volume of 50 sleeves with mounted photographs, some on verso. Main entry supplied from letter to the "Russkii Zagranichnyi Istoricheskii Arkhiv, Prague." The letter, which is translated by W. Brumfield, says, "for your information, I have in my possession a valuable album of photos of types of Russian colonists in America, compiled by a certain Geiman, at the request of Bakhmetev. Unfortunately, there is no duplicate." Also available as individual $8 \times 10$ photographic negatives at NYPL. Russkii zagranichnyi istoricheskii arkhiv v Prage. Russians- United States.

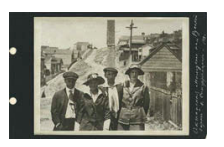

Slav Reserve (Photo) (Geiman. Tipy russkikh kolonistov) SLV 045 [Russian sectarians and farmers in North and South Dakota]; [Russian sectarians and farmers in North and South Dakota]; [Russian community in Los Angeles]; Russkie zemlekopy, Pensil'vanii; Russkie zemledel'tsy-Kolonisty v Nort-Dakotie iz Kievsk. gub.; Sem'ia narodnago uchitelia. Arizona; Dieti sirotek. Priiuta pri Pravaslavnom monastyrie. Shtat N'iu-Dzherzi; Sektanskaia molodezh' na "Russkii Gorkie" v Sanfrantsisko. 1918 g.; Dietskii sirotskii priiut Pravoslavnoi tserkvi v Sht. N'iuDzherzi; "Tainaia vechera” shesti apostolov sekty "Novyi Izrael”" San Frantsisko. 1917 g.; Obshchii zemlediel'cheskoi kolonii Russkikh sektantov; Tipichnyi domik za[..] tochnago russkago farmera; [Sectarian girl in the Russian community near Phoneix, AZ]; [Secterian farmers during a festival]; Shkola dlia dietei v Arizonskikh zemled. Koliniiakh (x Uchitel' - byvshii “diat'ka” iz Russkoi Armii); Russkoe rybatskoe poselenie v okrest. Los [Angeles]; Russki [...] na Tikho-Akensk. poberezh'e za rabotoi; Nashi rybaki v Kalifornii poslie udachnago ulova; [Funeral service at Russian Baptist church in San Francisco]; Ulichnaia zhizn' v Russko-Pol'skikh kvartalakh g. Chiga[go]; [Street life of Russian settlement in Chicago]; [Street life, Russo-Polish quarter, Chicago]; [Sectarian community in Los Angeles]; [Russian community in Los Angeles]; [Russian Jewish farm group near San Diego]; [Russian girl in Terra, IN]; [Russian groups in California, and at the Natural History Museum, NY, 1918]; [Russian musicians and poet]; [Russian miner, PA]; [Sectarian community in Los Angeles]; [Russian farmers, Fresno, CA]; [Dukhobory, Eire, PA]; ["Kievan" woman; Russian schoolgirls]; [Factory workers, Chicago; Russian girl in bathing suit, San Francisco]; [Russian sectarians from Uruguay working at a shipyard, San Francisco]; Syn' alkogolika. Baptistki; Russkiia zhenshchiny-farmershi iz okrest g. Feniks sht. Arizona; [Russian landlords]; [Two families of "New Israel" sect in Uruguay]; [Jewish farmer; Russian fishermen, CA]; ["Radical girl"; Russian-American girls]; [Russian "Bolsheviks" in CA]; [Russians in CA; picnic]; [Russian factory girls in Philadelphia]; [A Russian farmer in Arizona; 
Ukrainians in Bridgeport, CT]; [Russian soldier in American army, Panama Canal in 1915]; [Russian girls in CA. An unknown man]; [Farming land]; [German children colonists in ND]; [Polish emigres in Chicago]; [Russian workers at fish-cannery factory]; [Russian-German farmers, ND, 1915]; [Families in ND]; [German farmers in ND]; [Molokane, from Caucasus]; [Large group of men].

Gerbachevskii, Feodor Khudozhestvenno-fotograficheskii al'bom russkikh drevnostei i pamiatnikov pravoslaviia Kholmsko-Podliashskoi Rusi. Warsaw: [Feodor Gerbachevskii], 1891. 60 photographs on 56 leaves, $40 \times 30 \mathrm{~cm}$. "Wormer May 3, 1945." Stamp of A. Kantor, Perepletchik, v Varshave; library location marker reads: shkaf 12, polka I., No. 24-187. Binding, contemporary, of purple velvet, with white moire silk doublure; full gilt edges; with border and stamped in gold on cover.

Russkaia pravoslavnaia tserkov' - Poland. Podlasie (Poland: Region) Antiquities.

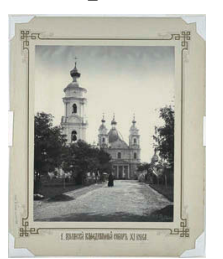

Slav. Reserve (Photo) (Gerbachevskii. Khudozhestvennofotograficheskii albom) SLV 046 [Cover title]; [Photo-montage page]; Kholmskii kafedral'nyi sobor XI veka; Vnutrennii vid Kholmskago sobora; Kholmskaia chudotvornaia ikona Bozhiei Materi; Bielavinskaia bashnia v 2 verstakh ot g. Kholma; Spasskaia tserkov', perestroennaia iz iazycheskago kapishcha; Stolpenskaia bashnia v odnoi verstie ot Spasskoi tserkvi; Vnutrennii vid Spasskoi tserkvi; Tserkov' Uspeniia Bozhiei Materi v g. Shchebreshinie (Liublin. gub); Vnutrennii vid Shchebreshinskoi tserkvi; Drevniia ikony Shchebreshinskoi tserkvi; Drevnii naprestol'nyi krest Shchebreshinskoi tserkvi; Dokument Shchebreshinskoi tserkvi 1593 g.; Liublinskaia SpasoPreobrazhenskaia tserkov'; Drevnii ikonostas Spaso-Preobrazhenskoi tserkvi; Liublinskaia chudotvornaia ikona Bozhiei Materi; Drevnii naprestol'nyi krest Spaso-Preobrazhenskoi tserkvi; Tserkov' Sv. Nikolaia v g. Liublinie X vieka, obrashchennaia v kostel v XVI viekie; Nikolaevskaia tserkov' v g. Samost'ie (Liubl. gub.); Drevnii ikonostas Nikolaevskoi tserkvi v gor. Samost'ie; Tsarskiia vrata Nikolaevskoi tserkvi; Blagoslovennaia gramota Ep. Gedeona Balabana 1606 g.; Drevnii krest Samost'skoi Nikolaevskoi tserkvi; Khramovaia ikona Voznesenskoi Samost'skoi tserkvi nynie ne sushchestvuiushchei; Stranitsa iz rukopisnago Evangeliia Samost'skoi Voznesenskoi tserkvi (v natural'nuiu velichinu); Selo Otroch Liublin. gub., gdie po predaniiu byl muzhskoi monastyr'; Dvie stranitsy iz apostola Krasnostavskoi tserkvi Liubl. gub. (skoropis' 1566 goda); Vid Mogol'nitskoi tserkvi i eia drevnii naprestol'nyi krest; Tserkov' Iablochinskago muzhskago monastyria. (Siedletskoi gub.); Obshchii vid Iablochinskago monastyria (s zapada); Vnutrennii vid tserk. Iablochinsk. monastyria; Rukopis' Ep. Paisiia, sokhranivshaiasia v naprestol'nom Evangelii Iablochinskago monastyria; 
Dve stranitsy iz rukopisnago evangeliia Iablochinskago monastyria, (v natural'n. velichinu); Dve ikony iz ikonostasa drevnei tserkvi Iablochinskago monastyria; Sviato-Dukhovskaia tserkov'v pos. Kodnie XV vieka (Siedlets. gub.); Sviato-Dukhovskaia tserkov' i zamok kniazei Sapiegov v p. Kodnie (s vostochn. storony); Vnutrennii vid Sviato-Dukhovskoi tserkvi; Nadgrobnaia plita Ioanna Sumeonovicha Sapiegi 1520 g. iz Sviato-Dukhovskoi tserkvi; Kodenskaia chudotvornaia ikona Bozhiei Materi (iz Sviato-Dukhovskoi tserkvi); Dereviannyia rieznyia podsviechniki iz Sviato-Dukhovskoi tserkvi i dve stranitsi iz Apostola Tsitsiborskoi tserkvi Siedletskoi gubern. (rukopis' v natural'n. velichinu); Vid prestola i kolokol'ni Sviato-Mikhailovskoi tserkvi 1500 g. v p. Kodnie; Naprestol'nyi krest Sviato-Mikhailovskoi tserkvi i naprestol'nyi krest i evangelie Sviato-Dukhovskoi tserkvi; Ikony Spasitelia i Bozhiei Materi iz ikonostasa Sviato-Mikhailovskoi tserkvi; Vid Sviato-Troitskoi Kodenskoi tserkvi, v XVII vieke obrashchennoi v kostel, nyne zhe pravoslavnoi; Vnutrennii vid Sviato-Troitskoi tserkvi; Vid kupola Sviato-Troitskoi tserkvi (vnutri); Liesninskaia chudotvornaia ikona Bozhiei Materi, nakhoditsia v Liesninskom zhenskom monastyrie Siedletskoi gub.; Ikona Bozhiei Materi i Tsarskiia vrata Ostrovskoi tserkvi 1276 goda (Siedletskoi gubernii); Ikona Spasitelia i tsarskiia vrata drevnei Kolekhovichskoi tserkvi (Siedlets. gub.); Krest iz tserkvi posada Sosnovitsy Siedlets. gub. (XV v); Chetyre stranitsy iz rukopisnykh evangelii: I) Korchevskago XV v., II) Rogoznenskago 1552 g. III) Kolembrodskago XV v., IV) Dolgovskago 1571 g.; Miesto drevnei kafedral'noi Ugrusskoi tserkvi v sele Ugrusske Siedlets. gub.; Ikona Bozhiei materi iz drevnei Ugrusskoi tserkvi; Ikony iz drevniago ikonostasa tserkvi v sele Sobiborie Siedlets. gub.; Tsarskiia vrata i ikony Dolgovskoi tserkvi 1651 g. (Siedlets. gub.); Miednyi krest XVI vieka, naidennyi v Ostrolenskom uezde Lomzhinskoi gubernii; Dve stranitsy iz Vishnitskago Naprestol'nago evangeliia 1575 g., Vilenskoe izdanie. (Siedlets. gub.).

Gospital' Krasnago Kresta pri Minskom Blag. O.vie "Dobrochinnost'.” 29 Maia 1916 g. 1 mounted photograph, $39 \times 26 \mathrm{~cm}$. Binder's title. "Perl. Jun 18, 1931." Inserted is a typewritten copy of an order issued by Grand Duchess Maria Pavlovna under no. 155 of June 3, 1916. From original portfolio. Rossiiskoe obshchestvo Krasnago kresta. Slavic Recon Project.

Metropolitan New York Library Council, 2008. World War, 1914-1918 Hospitals.

Slav. Reserve (Photo) (Gospital Krasnago kresta) SLV 047
[Binder's title]; [Medical staff and military officers].
Imperatorskaia okhota vo vladieniiakh Lovichskago kniaz-
hestva $\boldsymbol{v} \mathbf{1 8 8 4}$ g.

Warsaw: Tipografiia i slovolitnia S. Leventalia, 1886. 36 p., 5 leaves of plates, $30 \times 23.5 \mathrm{~cm}$. Chiefly photographs of hunting scenes and group 
portraits of the party of Alexander III, Emperor of Russia. Gift of Mrs. Marina Shelehoff, 1995.

Malykhin, Nikolai (compiler). Photographs taken by Brandel'. Alexander III, Emperor of Russia, 1845-1894. Hunting - Russia.

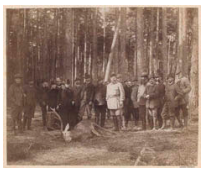

Slav. Reserve (Photo) 95-4599 (Malykhin) SLV 051 [Cover title]; [Alexander III and imperial family after a hunt, with deer on the ground]; [Empress Alexander III/Imperial family on horseback]; [Empress, a consort of Alexander III]; [Count Aleksandr Gustavovich Berg, and companies at hunting]; [Alexander III's breakfast in the forest/huntsmen and Russian nobelmen].

Istoricheskie vidy v gorodie Kostromie i selie Korobovie. [S.1.: s.n., 1878?]. 8 mounted photographs, $32 \times 42 \mathrm{~cm}$. "Perl. Jun 18, 1931."

Title from original portfolio. Handwritten signature on photographs: ot Z. I. Degtiareva. 16 avgusta 1878 g. Cover title. Slavic Recon Project. Metropolitan New York Library Council, 2008. Degtiareva, Z. I. Architecture - Russia (Federation) - Kostroma (Kostromskaia oblast). Kostroma (Kostromskaia oblast, Russia).

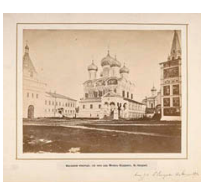

Slav. Reserve (Photo) (Istoricheskie vidy v gorodie Kostromie i selie korobovie) SLV 052 [Cover title]; Naruzhnyi vid tserkvi vo imia sobora Ioanna Predtechi, v selie Korobovie; Dvorets tsaria Mikhaila Fedorovicha, v Ipat'evskom monastyrie. V Kostromie; Pamiatnik tsariu Mikhailu Fedorovichu i grazhdaninu Ivanu Susaninu v Kostromie; Ipat'evskii monastyr', gdie zhil tsar' Mikhail Fedorovich. V Kostromie; Vnutrennii vid tserkvi vo imia sobora Ioanna Predtechi, v selie Korobovie; Rodina grazhdanina Ivana Susanina, Kostromskoi gubernii selo Korobovo; Vnov' ustroennyi sad pri Dvortsie Imperatora Petra Velikago, chto na Peterburgskoi storonie, 30 Avgusta 1875 goda; Vnov' ustroennyi sad pri Dvortsie Imperatora Petra Velikago, chto na Peterburgskoi storonie, 30 Avgusta 1875 goda.

Istoricheskoe opisanie postroeniia v Moskvie Khrama vo imia Khrista Spasitelia. Moscow: Tipografiia V. Got'e, 1869. 4 mounted photographs. $46 \times 33.5 \mathrm{~cm}$. One of 300 copies printed. Photographs by Sherer, Nabgol'ts i Ko (Sherer, Nabgol'ts and Co.). See also three titles by M. S. Mostovski, Istoricheskoe opisanie khrama vo imia Khrista Spasitelia v Moskve (Moscow, 1883); Istoriia Khrama Khrista Spasitelia v Moskve (Moscow, 1883); Khram Khrista Spasitelia v Moskve. Chtenie dlia naroda (Moskva, 1884). All three volumes bear the bookplate of Grand Duke Vladimir Aleksandrovich. "Perl. Jun 18, 1931." Khram Khrista Spasitelia (Moscow, Russia).

Slav. Reserve (Photo) (Istoricheskoe opisanie postroeniia v Moskvie khrama vo imia Khrista Spasitelia) SLV 053

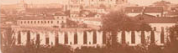
[Binder's title]; [Cover title]; Fasad Khrama, vo imia Khrista Spasitelia; Obshchii plan khrama, s mozaichnym polom; Razriez po linii A. B.; Glavnyi iconostas Khrama, vo imia Khrista Spasitelia. 
Iz moego al'boma: shest'desiat fotograficheskikh snimkov Eia Imperatorskago Velichestva Gosudaryni Imperatritsy Aleksandry Feodorovny. Petrograd: Izdanie T- va M.O. Volf., [1915?]. 20 leaves: (black $\&$ white, mounted); $21 \times 26 \mathrm{~cm}$. "Vsia chistaia pribyl" ot etogo izdaniia tsielikom postupaet v pol'zu ranenykh voinov"- T.p. Chiefly photographs of the family of Nicholas II.

Gift of Mrs. Marina Shelehoff, 1995. Nicholas II, Emperor of Russia, 1868-1918. Romanov, House of.

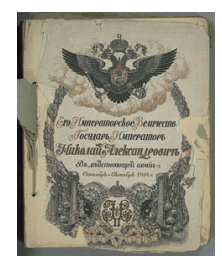

Slav. Reserve (Photo) 95-4592 SLV 054 [Cover title]; Marshrut pervago i vtorovo Vysochaishago puteshestviia. Sentiabr'Oktiabr' 1914; Spala. 1912 g.; Livadiia, 4 Oktiabria 1913 g.; Livadiia; Livadiia. Vid na Ialtu s Eriklinka; Livadiia; Krym; E.I. V. Velikaia Kniazhna Tat'iana Nikolaevna. 1914 g.; E.I.V. Velikaia Kniazhna Mariia Nikolaevna. 1914 g./Krym; Tsarskoe Selo. 1915 g.; 1915 g. Tsarskoe Selo; Imperatorskaia Iakhta "Shtandart” 1914 g.; Tsarskoe Selo. 1915 g.; E.I.V. Velikaia Kniazhna Anastasiia Nikolaevna. Tsarskoe Selo. 1915 g.; E.I.V. Velikaia Kniazhna Mariia Nikolaevna. Tsarskoe Selo. 1915 g.; Krym 1914 g./Na stantsii Al'ma; E.I.V. Velikaia Kniazhna Mariia Nikolaevna. Tsarskoe Selo. 1915 g.; Feodorovskii Sobor. Tsarskoe Selo; Tsarskoe Selo. 1915 g.; Tsarskoe Selo. 1915 g.; Tsarskoe Selo. 1914 g.; Slon. Tsarskoe Selo; Tsarskoe Selo. 1915 g.; Imperatorskaia Iakhta "Shtandart." 1913 g.; E.I.V. Velikii Kniaz' Mikhail Aleksandrovich. Tsarskoe Selo 1915 g.; E.I.V. Gosudar' Imperator i I.I.V. Velikiia Kniazhny Tat'iana Nikolaevna i Mariia Nikolaevna; Dvortsovyi Lazaret, Tsarskoe Selo. 1915 g./ Tsarskoe Selo; Dvortsovyi Lazaret. 1914 g.; I.I.V. Velikiia Kniazhny Ol'ga Nikolaevna i Tat'iana Nikolaevna. Livadiia 1913 g.; E.I.V. Velikaia Kniazhna Ol'ga Nikolaevna. Livadiia. 1914 g.; Tsarskoe Selo. 1914 g. 1915 g.; E.I.V. Velikaia Kniazhna Mariia Nikolaevna. Livadiia. 1914 g.; Imperatorskaia Iakhta "Shtandart." 1914 g.; Orianda. 1914 g.; I.I.V. Velikie Kniazhny Mariia Nikolaevna i Anastasiia Nikolaevna; Starshii vrach Dvortsovago Lazareta Kniazhna Gedroits; I.I.V. Velikie Kniazhny Ol'ga Nikolaevna i Tat'iana Nikolaevna i riadovoi bezdenezhnykh 110 piekh. Kamskago polka. Dvortsovyi lazaret, Tsarskoe Selo. 1914 g.; Dvortsovyi Lazaret. Tsarskoe Selo. 1914 i 1915 g.; E.I.V. Velikaia Kniazhna Ol'ga Nikolaevna. Shef 3-go Gusarskago Elizavetgradskago polka; E.I.V. Velikaia Knizhna Tat'iana Nikolaevna. Shef 8-go Ulanskago Voznesenskago polka. 1913; Smotr 1-go Leib Dragunskago Moskovskago Imperatora Petra Velikago polka. 1915; E.I.V. Nasliednik Tsesarevich, Vel. Kn. Ol'ga Nikolaevna i Tat'iana Nikolaevna. 1915 g.; E.I.V. Gosudarynia Imperatritsa, Vel. Kn. Ol'ga Nikolaevna i Tatiiana Nikolaevna. 1915 g.; I.I.V. Velikie Kniazhny Ol'ga Nikolaevna i Mariia Nikolaevna; Tsarskoe Selo. 1913 g. 
Izobrazheniia russkikh sviatykh: X-XVIII vek. [Russia?: s. n., 18-?] 20 photographs, $18 \times 26 \mathrm{~cm}$. Some photographs signed: N. Negodaev. Binder's title.

Bound in black grained morocco, gilt. With bookplate of Grand Duke Vladimir Aleksandrovich. "Perl. Jun 18, 1931." Metropolitan New York Library Council, 2008.

Slavic Recon Project. Mural painting and decoration-Russia. Christian saints-Russia. Negodaev, Nikolai Semionovich.

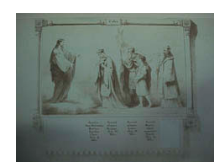

Slav. Reserve (Photo) (Izobrazheniia russkikh sviatykh) SLV 055 [4 saints: Sviataia Velikaia Kniaginia Ol'ga, Sviatyi muchenik Feodor and others, 969-992]; [32 saints: Sv. Vel. kn. Vladimir, Sv. kn. Boris and others, 1015-1098]; [31 saints: Sv. Nikon, Sv. Mark and others, 1101-1158]; [30 saints: Sv. Konstantin, Sv. Ioann and others, 1159-1199]; [23 saints: Sv. Dosifei, Sv. Avraamii and others, 1218-1257]; [17 saints: Sv. Evfrosinia, Sv. Kniaz' Aleksandr Nevskii and others, 1250-1299]; [Pri Igumenie Iosaafie Sviatogorskago Pskovskago monastyria, 1299, 8 saints]; [24 saints: Sv. Kn. Daniil, Sv. Maksim and others, 1303-1391]; [21 saints: Sv. Sergii Radonezhskii, Sv. Iakov and others, 13911398]; [28 saints: Sv. Agadon, Sv. Akila and others]; [24 saints: Sv. Andronii, Sv. Arsenii and others, 1402-1430]; [23 saints: Sv. Fotii, Sv. Maksim and others, 1433-1452]; [22 saints: Sv. Filipp, Sv. Tikhon and others, 1451-1480]; [19 saints: Sv. Evfrosin, Sv. Serapion and others, 1481-1497]; [26 saints: Sv. Tikhon, Sv. Pavsii and others, 1503-1552]; [26 saints: Sv. Kirill, Sv. Aleksandr and others, 1532-1561]; [25 saints: Sv. Khariton, Sv. Timofei and others, 1562-1600]; [15 saints: Sv. Nikandr, Sv. Sergii and others, 1603-1622]; [23 saints: Sv. Prokopii, Sv. Dionisii and others, 1624-1695]; [5 saints: Sv. Mitrofan, Sv. Dimitrii and others, 1703-1783].

K osviashcheniiu Chasovni Khrista Spasitelia v g. Kazani 21 Avgusta 1905 g. Kazan: A. Viatkina, [1905] 8 mounted photographs; $24.5 \times 34 \mathrm{~cm}$. Chasovnia Khrista Spasitelia (Kazan, Russia).

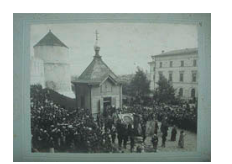

Slav. Reserve (Photo) 99-5214 SLV 056 [A view of an icon of Jesus the Redeemer in Kazan' City]; [A Church in Kazan' City]; [An Iconostasis in the Church]; [Ceremony of the Blessing of the Chapel]; [Ceremony of the Blessing of the Chapel]; [Ceremony of the Blessing of the Chapel]; [A view of the Church and the Chapel]; [A view of the Church and the Chapel].

Kanikoff, Ivan. [Photograph, 1861].

St. Petersburg: Louis d'Eichthal, 1861. 1 photograph. $38 \times 28 \mathrm{~cm}$. Oval photograph of samoed Ivan Kanikoff (Kanikov) by the St. Petersburg photographer Louis d'Eichthal in 1861. Eichthal, Louis d', photographer. 


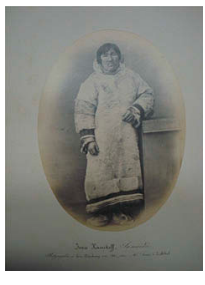

Slav. Reserve (Photo) 95-1012 SLV 057

Karelin, Andrei Osipovich, 1837-1906. Khudozhestvennyi al'bom fotografii s natury, khudozhnika Karelina, fotografa Imperatorskoi Akademii khudozhestv. Nizhny Novgorod, [1885]. 14 mounted photographs: ill., ports.; $47.5 \times 34.5 \mathrm{~cm}$. Cover title. Issued in red leather portfolio. Printed on the first plate: "Ego Imperatorskomu Vysochestvu Velikomu Kniaziu Vladimiru Aleksandrovichu na pamiat' o posieshchenii Ego Vysochestvom Nizhegorodskoi kustarno-promyshlennoi i sel'sko-khoziaistvennoi vystavki 1885 g. Vsepoddannieishe podnosit khudozhnik-fotograf A. Karelin.”

Metropolitan New York Library Council, 2008. Slavic Recon Project. Russians - Portraits. Costume - Russia.

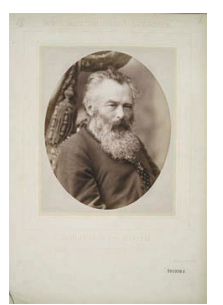

Slav. Reserve (Photo) (Karelin. Khudozhestvennyi albom fotografii s natury) SLV 058 [Cover title]; [Dedication page].; [A portrait of an old woman]; [A man with a goblet]; [A a couple with the woman seated]; [A couple in front of a window]; [A woman halfseated on a chaise-longue in front of a window]; [Portrait of Ivan Shishkin]; [A portrait of a priest with manuscripts]; [A portrait of a mother reading with two children]; [A portrait of a man with dog at his feet]; [A portrait of a woman with a book]; [A portrait of Russian priest]; [A portrait of V. I. Yacobi]; [A portrait of a man from behind]; [A portrait of D. I. Mendeleev].

Karelin, Andrei Osipovich, 1837-1906.

Khudozhestvennyi al'bom fotografii $s$ natury, khudozhnik $i$ fotograf Imperatorskoi Akademii khudozhestv Karelin.

Nizhny Novgorod, [18-]. 25 mounted photographs, 42.5 x $33 \mathrm{~cm}$. Covertitle. Issued in red leather portfolio.

Each plate captioned: "Khudozhestvennyi al'bom, fotografii s natury, v Nizhnem Novgorode, khudozhn. A. Karelin.”

"Partial album originally bound in red leather with gilt letters, now disbound and boxed in original cover bearing the title. 25 albumen prints ( $\pm 9 \times 6$ ") mounted on elaborately designed mounts, repeating the title and the place and adding in lower left corner: Artist A. Karelin. The prints are numbered in pencil, include posed studies of mostly young women, alone, in couples, or in groups, visiting an artist's studio, playing games, reading or looking at photographic albums, etc. Also family groups and a few portraits of 'types', beggars, peasant girls in costume. The majority of scenes are posed in comfortable, fashionable middle-class interiors (there are a few outdoor scenes) aiming to evoke the idyllic pleasures of youth, innocence, and family life. There is a conspicuous lack of naturalness in these 'tableaux' but a beautiful sense of light, an empathy for the young, and some striking portraits (no. 11,20). This and similar work established Karelin's reputation as the preeminent Russian artistic photographer. The condition of the prints is good but many have yellowed. The mounts are very 
brittle at the covers. They should be encased in new mounts." (Pierre Apraxine) "Perl. Jun 18, 1931" on back of no. 1.

DOE Title II-C "Russian Illustrated Books and Photographs" Project. Russians. Costume-Russia.

Slav Reserve (Photo) (Karelin. Khudozhestvennyi albom). SLV 059 [Cover title]; [Two girls embracing]; [A woman with needlework]; [A family in a living room]; [A young woman wearing a hat]; [A girl wearing a straw hat]; [A portrait of a couple, doubled in the mirror]; [A portrait of two young women, sitting by a mirror]; [A portrait of a mother embroidering, and two children]; [A group of four women looking at prints]; [A young girl]; [A woman playing a guitar]; [A father and a young child]; [A peasant girl]; [A group playing blind man's buff]; [Two young women looking out of a window]; [A couple with a child standing on stairs]; [Three girls looking at photographic albums]; [A family in an interior]; [A woman giving charity to a begger and child]; [A woman in costume, embroidering]; [A scene at the window]; [A portrait of an old beggar and child]; [A peasant girl]; [A family group in an interior]; [A group of four children with a book].

Kavkaz. Souvenir du Caucase. [S.l.: s.n., 18-?] 25 photographs, 19 x $14.5 \mathrm{~cm}$. Cover title. Captions in Russian, French, and German.

Metropolitan New York Library Council, 2008. Slavic Recon Project. Caucasus-Pictorial works.

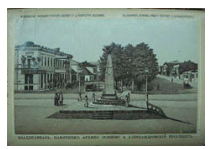

Slav. Reserve (Photo) (Kavkaz) SLV 060 Vladikavkaz, pamiatnik Arkhipu Osipovu i Aleksandrovskii prospect; Tiflis Teatr; Tiflis, Botanicheskii sad, razvaliny bashen' i kreposti; Tiflis, Metikhskii zamok; Tiflis. Grobnitsa Griboiedova u tserkvi Sv. Davida; Tiflis.

Tserkov' Sv. Davida; Tiflis, Gostinnitsa Kavkaz i Dvortsovaia ulitsa; Tiflis, Khram Slavy i ugol Bariatinskoi ulitsy; Tiflis, Veriiskii i Nokolaevskii most; Tiflis. Pamiatnik Kniaziu Vorontsovu; Tiflis, Golovinskii prospect; Voen.Gruz. dor. Mtskhet Byvshaia stolitsa Gruzii; Voen.-Gruz. dor. Gorod Dushet; Voen.-Gruz. dor. stantsiia Ananur i tserkov' V stolietiia; Voen.-Gruz. dor. stantsiia Pasanaur i tserkov'; Voen-Gruz. dor. Pasanaurskii shlagbaum; Voen.-Gruz. dor. pamiatnik poety A. Kazbeky; Voen.-Gruz. dor. gora Kazbek; Voen.-Gruz. dor. mosty v Dar'ial'skom uchshel'ie cherez rieku Terek; Voen.-Gruz. dor. Dar'ial'skoe ukrieplenie i zamok tsaritsy Tamary; Voen.-Gruz. dor. Giuletskii most; Voen.-Gruz. dor. doroga v Dar'ial'skom uchshel'e; Voen.-Gruz. dor. post redant, doroga k Larsu rieka Terek; Voenno-Gruzinskaia doroga. Ol'ginskii most; Vladikavkaz. Dvorets i sobor.

Kennan, George (1845-1924), compiler. The Caucasus; an album of photographs collected by George Kennan. [S. 1.: s. n., 187-?] 271 mounted photographs (some col.), $34 \times 28.5 \mathrm{~cm}$. The typewritten captions under the pictures have been copied from the back of the photographs, the inscriptions 
being for the most part in Mr. Kennan's own hand. These photographs were presented to The New York Public Library by Mrs. George Kennan in 1927.

Metropolitan New York Library Council, 2008. Slavic Recon Project. Kaxeti (Georgia).

Voenno-Gruzinskaia doroga (Georgia and Russia).

Architecture - Caucasus. Dagestan (Russia) - Description and travel. Tbilisi (Georgia) - Description and travel. Caucasus.

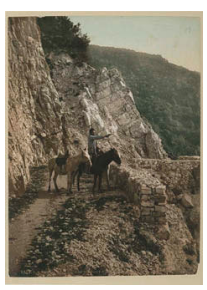

Slav. Reserve (Photo) (Kennan. the Caucasus) SLV 061 VoennoGruzinskaia doroga. Vladikavkaz, Ol'ginskii most i Stolovaia gora/ Vladikavkaz, Dvorets, skver i sobor; Voenno-Gruz. doroga. Vladikavkaz, Aleksandrovskii bul'var; Aziiatskii obied v dukhanie; Voenno-Gruzinskaia doroga. Iamshchiki/VoennoGruzinskaia doroga. Post Redant; [Terek]/[Voenno-Gruzinskaia doroga. Trusovskoe...]; Voenno-Gruzinskaia doroga. Otviesno visiachie kamni u Belty; Voenno-Gruzinskaia doroga, st. Balta; VoennoGruzinskaia doroga. Ushchel'e i doroga u bashni starago Larsa/VoennoGruz. doroga. Dar'ial'skoe ushchel'e; [?]/Voenno-Gruz. doroga. Dar'ial'skoe ushchel'e i selenie Giulety; Voenno-Gruzinskaia doroga. Dar'ialskoe ushchel'e, gruda kamnei; Voenno-Gruzinskaia doroga. Pamiatnik poetu A. Kazbek/Voenno-Gruzinskaia doroga. Dom i tserkov' dvorian Kazbek; [Fortress of All Saints near Kazbek]/Voenno-Gruzinskaia doroga, Gora Kazbek; Voenno-Gruzinskaia doroga. Ts. Sameba u podoshvy g. Kazbek/ [Beginning of Gorge of Darial from station of Kazbek]; VoennoGruzinskaia doroga. st. Kazbek; Voenno-Gruzinskaia doroga. Kazbek, tserkov' i stantsiia/Voenno-Gruzinskaia doroga. Kazbek, selenie i tserkov'; Voenno-Gruzinskaia doroga. Kazbek i tserkov'; Voenno-Gruz. Doroga. Kuralgin most. Raschistka zavala s gallerei i puti; Voenno-Gruzinskaia doroga. Razrabotka transhei dorogi u vala Bodo; Voenno-Gruzinskaia doroga. Pochtovaia [...]; Kavkaz u Bagatskago mosta; Voenno-Gruzinskaia doroga. Kolok. ts. Sameba u podosh. g. Kazbek; Voenno-Gruzinskaia doroga. Kazaki, okhraniaiushchie ukrieplenie/Voen.-Gruz. dor. Baidarskii most i saklia iz kotoroi zvoniat pri [...]; Voenno-Gruz. dor. Most chrez r. Terek. GorisTsikhi i gora Sion/Voenno-Gruz. doroga. Ushchel'e mezhdu Sionom i Kazbekom; Voenno-Gruzinskaia doroga. Sv. Sion i Bielaia tserkov'; Voen.Gruz. dor. Selenie Tserkov' (bielaia tserkov'); Voenno-Gruzinskaia doroga. st. Kobi; Voenno-Gruzinskaia doroga. Kobi. Razvaliny tserkvi Sviatogo Georgiia; Voenno-Gruzinskaia doroga. Tonnel' mezhdu st. Kobi i Gudauri; V.-G. D. Obshchii vid krestovoi gory na vysotie 1136 futov; VoennoGruzinskaia doroga. Gudaurskii pereval; Voenno-Gruzinskaia doroga. Chertova dolina: ot kresta k Gudauram; [Georgian Military Road. Station of Gudauri]; Voenno-Gruzinskaia doroga. Giuletskii most/VoennoGruzinskaia doroga. Giuletskii most i storozhevaia budka; VoennoGruzinskaia doroga. Bashnia kniazei Baratovykh mezhdu stantsiami Mlety 
i Pasanaur; Voen. Gruz. Dor. Fontan na Mletskom pod'emie; VoennoGruzinskaia doroga. Gallereia ot sniegovykh zavalov; Voenno-Gruz. Dor [...]; Voenno-Gruzinskaia doroga. Mletkii pod'em; Voenno-Gruzinskaia doroga [...]/Voenno-Gruzinskaia doroga. Mlety, gruzinskii dukhan; VoennoGruzinskaia doroga. Tserkov' vo imia Sv. Georgiia bliz stan. Mlety; [...]; [Aul Mleti]; Voenno-Gruzinskaia doroga. Skala mezhdu Pasanarom i Meltom; Voenno-Gruzinskaia doroga. Dzharakhovskoe ukrieplenie/ Voenno-Gruzinskaia doroga. Ushchel'e i derevnia mezhdu Pasanaurom i Mletami; Voenno-Gruzinskaia doroga. Miestonst' bliz Pasanaura; [Georgian Military Road. The Pasanauri Toll-Gate]; Voenno-Gruzinskaia doroga. st Pasanaur; Voenno-Gruzinskaia doroga. Otvies skala mezhdu stantsiiami Pasanarie i Ananurie; Voenno-Gruzinskaia doroga. st. Dushet; Voenno-Gruzinskaia doroga. gor. Dushet; Voenno-Gruzinskaia doroga. Bielyi zamok v Dushetie; Voenno-Gruzinskaia doroga. Dukhan Chartaly mezhdu Anan. i Pasanaurom; Voenno-Gruzinskaia dor. Ananur. Ulitsa i tserkov' V-go stolietiia/Voenno-Gruzinskaia doroga. Dukhan Charta; Voenno-Gruzinskaia doroga [...]; Voenno-Gruzinskaia doroga. st. Mtskhet; Voenno-Gruzinskaia doroga. sel. Mtskhet. Byvsh. stolitsa Gruzii; VoennoGruzinskaia doroga [...]/Voenno-Gruzinskaia doroga. Mtskhet, krepost' "Natskher"; Voenno-Gruzinskaia doroga. Mtskhet, sobor/[...]; Voen.Gruzin. dor. Mtskhet Monastyr' Sv. [...]; Voenno-Gruzinskaia doroga [...]; Voenno-Gruzinskaia doroga. Zapadnaia stiena mtskhetskago sobora; Tiflis, krepost' i gorod; Obshchii vid Tiflisa; Obshchii vid Tiflisa; Obshchii vid Tiflisa; Vid Tiflisa s Peski; [View of Tiflis from the Peski looking toward the church of St. David]; [Tiflis: Metekhski prison from the Avlabar Bridge]; [Tiflis: River Kur and the old fortress]; [Tiflis: The old fortress]; [Tiflis: The old town]; [Tiflis: Michael Bridge and Madatofski Island]; [Tiflis: Botanical Garden (cultivated part)]; [Tiflis: The Imperial Theatre]; Tiflis, Erivanskaia ploshchad'; Tiflis; Golovinskii prospekt v Tiflise; Tiflis. Dvorianskoe Sobranie, tsarskii vkhod na bal (29-go sent. 1888 g.); Tiflis. Golovinskii prospect ot Khrama Slavy v Verie; Tiflis. Khram Slavy. Golovinskii prospekt i ugol Bariatinskoi ul.; Tiflis [...]; [Tiflis: Square on the "Peski"]; Tiflis [...]; Tiflis. [...] otviesnaia skala u Metekhskago zamka; [Tiflis: Metekhskii castle and River Kura]; [Tiflis: The Avlabar Bridge]; Avlabarskii most.; [Tiflis: Ravine on the southern side of the Botanical Garden]; [Environments of Tiflis. Down Koora river.]; Tiflis. Dukhovnaia seminariia na Erivanskoi ploshchade; [Tiflis: The Orthodox Theological Seminary from the side of the Soldier's Bazaar]; Tiflis. Gostinitsa "London"; [Tiflis: Zion Cathedral from the river Kur]; Tiflis. Orbeliianovskiia siernyia bani; Tiflis. Monument kniazia Vorotsova na Mikhailovskom mostu; [Tiflis: Church of St. David]; Tiflis. Sv. David i dorozhka zigzagami k nemu; Tiflis. Grobnitsa Griboiedova u tserkvi Sv. Davida/Lavochka, Persidskaia apteka; Tiflis. Bannaia ulitsa; [Tiflis: General view of the old fortress]; Tiflis. Obshchii vid razvalinyi 
krieposti i r. Kura; Tiflis. Razvaliny krieposti i chast' goroda; [Tiflis: Ruins of the old Fortress]; [Tiflis: Part of the old city]; [Tiflis: Part of old city. Street on right leads to Botanical garden and on the left to Sulphur Baths]; [Tiflis: Street toward Botanical Garden]; Tiflis. Sv. David s Laboratornoi ulitsy./ Tiflis. Bannyia ulitsa [...]; Tiflis. Staraia chast' goroda u ban'; Tiflis [...]; [Tiflis: Part of old city near the Sulphur Baths]; [Tiflis: Tatar bazaar]; [Tiflis: Tatar square and bazaar]; Tiflis. Vokzal; Tiflis. Mechet' na maidanie i chast' predmiestie goroda Sololaki; [Tiflis: Bridge to the Tatar bazaar]; [Tiflis: The Botanical garden from the S.E. side]; [Tiflis: The Botanical Garden (looking more to the Southward)]; [Tiflis: the Botanical garden from the E. side]; [Tiflis: Waterfall on Western side of Botanical Garden]; Tiflis. Vodopad $\mathrm{u}$ Botanicheskago sada; [Tiflis: Bridge in the Botanical Garden]; Tiflis. Botanicheskii sad, Dekorativnyi otdiel; [Tiflis: Palace Street from Eriwan Square]; [Environments of Tiflis. One sees a water wheel irrigation the gardens of Ortachalee and a floating mill.]; [Environments of Tiflis. Plavuchaia mel'nitsa i krut]; [Environments of Tiflis. Mnatsakani Bridge on Koora suburb Ortachalee]; [Tiflis: Types. A camel cart]; [Tiflis: Shop for sale of Caucasian silversmith's work]; Arba s paroi buivalov i paroi bykov v upriazhie; [A Caucasian]; Prodavets [...]; Tiflis, karavan verbliudov prigotovlenie $\mathrm{k}$ vykhodu; Molokanskaia derevnia i molokane na ulitse; [A Molokan wagon (fourgon)]; Kinto s lotkom na golovie; [Tiflis types Armenian and Persian porters]; Pers ulichnyi torgovets; [...] Vavskii armianskii; [Tiflis types. Bread bakers]; Molokane; Persid. chernorabochiia; Tiflis. Pochetnaia strazha (kn. Argutinskii i dr.); Gruzinki s dairoi; [Tiflis types. Dealers in fruits/An Armenian woman]; [Tiflis types. A street fruit seller]; Gruzinki; Prodavets gruzinskago chureka; Kakhetinets s burdiukom, kuvshinom v dva riada i azarieshi iz roga/Sazandari, Kavkazskii orkestr; [Tiflis types. A Georgian woman]; Mushi nosiashchiia tiazhesti; Osel s siedokom armianinom i koshelki s ptitsei; [A Georgian woman/Tiflis types: An Armenian woman]; Gruzin i gruzinka; [Tiflis types. Armenian women of poorer class]; Pers i osel nav'iuchennyi mieshkami s samanom; Tiflis. Persy, prodavtsy kovrov; [Tiflis types. A street shoe-cobler./Tiflis types. Persian porters]; Musha s burdiukom/Tulugchi (vodovoz); Prodazha drov na oslie; Tiflis. Most [...]; [Daghestan. Argutinskaya street, Temir-Khan-Shura]; Bazar v Temir-Khan-Shurie; Pamiatnik, "Apsherontsam za Gunib” v T-KhShurie; Gor. Temir-Khan-Shura, s Kavalerie-Batarei; [Daghestan. The upper boulevard, Temir-Khan-Shura]; Pamiatnik kn. Argutinskomu v T-Kh-Shurie; [Daghestan. Consecration of the monument erected over the rock on the summit of Gunib where Prince Bariatinski received the surrender of Shamyl]; Pamiatnik nad "Kamnem Kn. Bariatinskago" na Gunibie; Gor. Temir-KhanShura [...]; Muselim'-aul'skii most bliz T.-Kh.-Shury; [View of town near Temir-Khan-Shura]; [View of town near Temir-Khan-Shura]; Uvr. Evgenievskoe i most cherez r. Sulak T.-Kh.-Shur okr.; Aul Ishkarty, T.-Kh.- 
Shurinskago okr.; Ishkarty, byvsh. Sh.-Kvartira Apsheronskago polka; [Daghestan. Regiment camp]; [Daghestan. Daghestan horsemen on their way to camp]; Aul Atlybuiun mezhdu g. T.-Kh.-Shuroiu i Petrovskom; [Daghestan. Laborers at the Sulphur mine of Khiyut]; Vid gor. Petrovska s moria; Vid gor. Petrovska s moria; [Daghestan. Steamer "Grand Duke Alexei" at Petrovsk]; Gor. Petrovsk, Dagestanskoi oblasti; Most bliz aula Gimry, Dagestanskoi oblasti; Arakanskii most, Avarskago okruga; Arakanskoe ushchel'e, Avarskago okruga; [Daghestan. Aul Kikuni]; Aul Untsukul, Avarskago okruga; [Bridge around Avarskii okrug]; Tlokhskii most, cherez rieku Koisu, Avarskago okruga; Gora "Akhul'go" (byvsh. Ukr. Shamilia), Avarsk. okr.; [Daghestan. Ruins of a Christain church near village of Datun]; [Daghestan. Manoeuvres of infantry and artillery near Khunzakh]; [Daghestan. Manoeuvres of troops near Khunzakh]; [Daghestan. Gorge of Akhulgo]; Ukr. Botlikh, Dagestanskoi oblasti; Aul Botlikh, Andiiskago okr. Dagestanskoi obl.; Preobrazhenskii most. Andiiskago okruga; [Daghestan. Approach to the peak of Gunib]; [Daghestan. Middle terrace. Peak of Gunib]; Razvaliny aul Shamilia na verkhnem Gunibie; Ploshchad' Nizhniago Guniba; Ploshchad' Nizhniago Guniba; [Photograph of a painting of a group of troops]; Slantsovoe ushchel'e Gunibsk. okr.; [Daghestan. Gorge in neighborhood of Gunib]; Zavtrak v Slantsovom Ushchel'ie, E. I. V. Vel. Kn. Georgiia Aleksandrovicha, v 1893 g. Fot. I. Abuladze; Lager. Ego Imperatorskago Vysochestva Velikago Kniazia Georgiia Aleksandrovicha na Gunib v 1893 g.; Lager' Ego Imperatorskago Vysochestva Velikago Kniazia Georgiia Aleksandrovicha na Gunibie; Lager' konvoia soprovozhdavshago E. I. V. Vel. Kn. Georgiia Aleksandrovicha na verkh. Gunibie; Vyiezd E. I. V. Vel. Kn. Georgiia Aleksandrovicha iz Guniba, v 1893 g.; Nizhnii Gunibskoi most; [Gunib]; [Georgievskii Bridge]; Ukr. Kazikumukh Dag. obl.; Tsudakharskoe Ushchel'e, Darginskago okruga; Razvaliny aula Tsudakharie, Darginskago okruga; Khodzhal-Makhinskii most, Darginskago okruga; [Daghestan. Man of Khodjal-Makhi]; Mel'nitsy na riekie Iakhsu v Cherkeie. Ter. obl.; Vodopad v aulie Cherkei, Terskoi oblasti; [Daghestan: View of a city]; [Daghestan: View of village]; [Daghestan. Dance the "Lezginka" Greater Kazanishchi]; [Daghestan. Avarian girl going for water/Daghestan. An old Avar woman]; [Daghestan. A Kumik family/ Miscellaneous. Returen of Lezghians from a raid]; [Miscellaneous. Khevsurs in camp/Warior]; Pastukh gruzin; Gruzinskaia arba otpravlenie na bogomolie; Kakhetiia. Arba dlia perevoza samanu; Arby dlia podvoza vinograda v imienii Kn. Mukhranskago; Kakhetiia, venakhivanie zemli; Kakhetiia, venakhivanie zemli; Kakhetiia, Kuvshiny (kvevri), v kotorykh sokhraniaiut vino; Kakhetiia, pechenie khlieba $\mathrm{v}$ tornie (pekarnia); Gruzinskaia arba. Otpravlenie na bogomolie; [Kakhetiia: a boy with cow]; [Kakhetiia: Georgian women]; [Kakhetia. Georgian house and stable]; [Kakhetia. Georgian threshing machine and mill]; Kakhetiia. Imienie grafa 
S. D. Sheremeteva, terrasa i vid na dolinu r. Alazanv [...]; Kakhetiia. g. Signakh ot kriepostnykh vorot; [Family and servants of a priest in Kakhetia]; [Kakhetiia. Count Sheremetev's house]; [Kakhetiia: Church and people]; Kakhetiia g. Signakh ot monastyria Sv. Niny; [Kakhetia: castle]; [Kakhetia. Road to Kakhetia across Gomber]; [Kakhetia. Road to Kakhetia across Gomber]; [Kakhetia. Town of Signakh]; [Kakhetia. Kabenski monastery; erected in 13th cent. by Queen Tamara]; [Kakhetia. Ruined Monastery of St. Nina near Kardanakhi]; Zangezuroskago uiezda Miestechko [...]; [Western Georgia. Kutais]; [Western Georgia: Kutais]; [General view of Kutais]; Kutais. Obshchii vid goroda; Kutais. Razvaliny khrama Bagratov; Kutais. Ferma, rachinskaia doroga. r. Rion i v dali monet. Gelaty; Ambar, sklad dlia kukuruzy; [A Mingrelian Cart]; [Kutais. Country house]; Zugdidy (mingreliia) arba v upriazhi buivoly; [Batum. People]; [A Borchalinski Tatar selling green herbs from the country]; [Emigration of Armenians into Georgia during the Russo-Turkish war of 1878-9]; [Batum. View of a village]; [Batum. A man in uniform]; [Fortress]; [Square in Gunib]; [General view of a town]; [Soldier/Musician of Daghestan calvary regiment]; [Festival/Uniform]; [General view of city].

Kennan, George (1845-1924), compiler. Portraits of Russian political exiles and convicts, with some additional photographs depicting the life of both political and common criminals in Siberia. Collected and presented to the New York Public Library by George Kennan. [Russia?, 188-]. $36.5 \times 28 \mathrm{~cm}$. Photographs mounted and bound. 246 albumen photographs, mostly carte de-visites, mounted in alphabetical order of the sitters. "The text of the legends placed below the photographs or on the opposite page consists of a copy of the inscription on the back of the photograph, coming in most cases from Mr. George Kennan's pen, with additional notes supplied by Mr. Konstantin M. Oberuchev and the Slavonic Division. Mr. Kennan's and Mr. Oberuchev's notes are followed by letters $\mathrm{K}$ and O respectively."

Political prisoners - Russia. Siberia (Russia). Prisoners (Political), Russia.

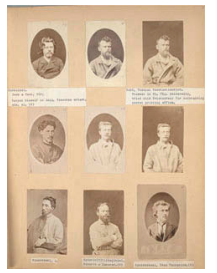

Slav. Reserve Photo (Kennan, G. Portraits of Russian political exiles and convicts) SLV 062 Table of contents [typed on inside cover]: George Kennan. Tomsk, Western Siberia, August 1885; The exiled bell in Uglich; Fialka, Izmailova, Spiridonova, Yaros, Bitzenko, Yezerskaya; Lazaref, Shishko, Fanny Moreinis and others. Exiles in Chita; Sternberg, Tetrajima, Orzhikn, Russel, B. Pilsudski, Vadetzki; Aleksandrov; Alekseyev, Piotr Alkeseyevich, 18511891; Alekseyeva, O.; Aptekman, Osip Vasil'yevich, 1849-; Armfeld, general'sha; Berdnikov, In Kara-Katorga; Bogomoletz, Sof ya; Bovbelski, Sept. 1880; Bukh, Nikolai Konstantinovich; Bukh, Nikolai Konstantinovich; Bulanov, Leonid; Butzinski, Dmitri; Butzinski, Dmitri; Byaloveski, A.; Bydorin (Bidarin); Byelokonski, Ivan Petrovich; Charushin, N.; Charushin, N.; Charushina, Anna Dmitriyevna, née Kuvshinskaya; Chernavski, Mikhail; Chernyshevski, Nikolai Gavrilovich, 1828-1889; Chikoidze, Mikhail 
Nikolayevich; Chudnovski, Solomon Lazarevich, 1850-; Chudnovski, Solomon Lazarevich, 1850-; Danilovich, Dr.; Degayev, Sergei P.; Dikovski; Dmokhovski; Mikhailova, Henrietta Nikolayevna, 1863; Dolgushin, Aleksandr Vasil'yevich; Doller; Dostoyevski, Fyodor Mikhailovich, 18221881; Dzhabadari, Ivan Spiridonovich, 1852-1913; Falinski; Fedorova, Yustina Nikolayevna; Filipova, Vyera Nikolayevna, née Figner; Filipova, Vyera Nikolayevna, née Figner; Filipova, Vyera Nikolayevna, née Figner; Frank, Roza; Franzholi, Mrs.; Franzholi; Fresser; Gamkrelidze, M.; Gerasimov, Tz.; Golubyatnikov, Dmitri; Ivanov, P.; Ivanova-Boreisha, Sofya Andreyevna; Kardashev, Stepan; Kastetzki; Kastuyrin, V.; Kizer; Klementz, Dmitri Aleksandrovich, 1848-1914; Klenov, Vasili; Klimenko, Mikhail; Kobylyanski, Lyudvig; Kobylyanski, Lyudvig; Kohan-Bernstein, Lev Matveyevich; Kohan-Bernstein, Lev Matveyevich; Kolyenkina, M.; Konov, Piotr; Pribveleva, Anna Pavlovna, née Korba, 1849-; Korolenko, Vladimir Galaktionovich; Kovalik, Sergei Filipovich, 1846-; Kovalevskaya, Mariya Pavlovna, née Vorontzova, 1849-1889; Koval'skaya, Yelizaveta Nikolayevna; Krivoshein; Krizhanovski; Kropotkin, Aleksandr Alekseyevich, 1841-1886; Post-mortem photograph of A. A. Kropotkin; Kropotkin, Piotr Alekseyevich, 1842-; Kropotkin, Piotr Alekseyevich, 1842-; Krukovskaya, Yu.; Khrushchov; Kvyatkovski, Aleksandr Aleksandrovich; Lazarev, Yegor Yegorovich; Lazarev, Yegor Yegorovich; Lesevich, Vladimir Viktorovich, 1837-1905; Lesevich, Vladimir Viktorovich, 1837-1905; Lewandowska, Leonarda; Lobanovski, P.; Liogki; Logovski, Aleksandr; Machtet, Grigori Aleksandrovich, 1852-1901; Machtet, Yelena, née Medvyedyeva; Machtet, Yelena, née Medvyedyeva; Machtet, Yelena, née Medvyedyeva; Makarevski, Aleksei; Malavski; Mantzevich, Mikhail; Martynov, Dr.; Martynova, Madame; Martynovski, Sergei Ivanovich, 1860-; Maslov; Meier; Merkhelev, Nikolai; Mikhailov, Adrian; Minakov, Ye.; Myshkin, Ippolit Nikitich; Myshkin, Ippolit Nikitich; Myshkin, Ippolit Nikitich; Neustroyev, Konstantin Gavrilovich, 1858-1883; Neustroyev, Konstantin Gavrilovich, 1858-1883; Nikitina, Sophia; Obnorski; Orlov, Pavel; Osipov, Tz.; Pashkovski, Tit.; Pavlov; Pekarski; Plotnikov; Potanin, Grigori Nikolayevich, 1835-; Potanin, Grigori Nikolayevich, 1835-; Preobrazhenski; Prikhodko; Revitzki; Rogachov, Dmitri Mikhailovich; Rubanchikova; Sadovnikov; Salova, Nionila Mikhailovna; Salova, Nionila Mikhailovna; Saltykov, Mikhail Yevgrafovich, 1826-1889; Sazhin, Mikhail; Semyanovski, Yevgeni Stepanovich; Shadrin; Shamarin; Sheffer, Yegor; Sheftel, Vera; Shmidova, Rebbeka; Shtromberg, Alexander Pavlovich, Baron; Sigida, Nadezhda Konstantinova; Sigida, Nadezhda Konstantinova; Sipovich, Aleksandr; Sipovich, Aleksandr; Sokolov, Nikolai Dmitriyevich; Sytzyanko; Stanyukovich, Konstantin Mikhailovich, 1844-1903; Starynkevich, Ivan Yul'yevich; Studzinski; Sukhomlin, Vasili Ivanovich; Sukhomlin, Vasili Ivanovich; Sukhomlin, Vasili Ivanovich; Sukhomlina, Madame; Sukhomlina, Madame; Svyetich; 
Syeryakov; Tereshchenko, Sergei Nikolayevich; Tikhonov - 1882; Trigoni; Trigoni; Troshchanski, Vasili; Troshchanski, Vasili; Tzitzenko, Ivan; Tzitzianov, A., knyaz'; Tzukkerman; Tzukkerman, Lazar; Tzukkerman, Lazar; Uspenskaya, Aleksandra Ivanovna, née Zasulich; Uspenskaya, Aleksandra Ivanovna, née Zasulich; Uspenski, Piotr Gavrilovich; Uspenski, Piotr Gavrilovich; Veimar, Orest Edward, 1845-1885; Veimar, Orest Edward, 1845-1885; Vitashevski, Nikolai Alekseyevich, 1857-; Vlastopulo, N.; Voinaralski, Porfiri Ivanovich, 1844-1898; Volkhovskaya, Mariya Yosifovna, née Antonova; Yadrintzev, Nikolai Mikhailovich, 1842-1894; Yakimov, Martyn; Yakubovich, Piotr Filippovich, 1860-; Yatzevich; Yokhel'son, Veniamin; Yonov, Vsevolod Mikhailovich; Zabaluyev; Zabaluyev; Zatzyepina, Zinaida; Zdanovich, Georgi Feliksovich; Zdanovich, Georgi Feliksovich; Zhebuniov, Leonid; Zhebuinov, Leonid; Zhebuniov, Sergei; Zlatopol'ski, Lev Solomonovich; Zotov, Nikolai L'vovich; Zotov, Nikolai L'vovich; Zubovski; Zundelevich; [14 unidentified]; Siberian convict said to be more than 100 years old in 1885; Three brodyags; Convicts and exiles going on board prison barge at Tiumen, 1885; Etape, or exile station house; Old and sick convicts in carts. Western Siberia, 1885; Convicts buying food from peasant women at a halting place on the Great Siberian road, 1885; Group of exile women and children at forwarding prison, Tiumen, 1885; A group of Siberian convicts on their way to the mines, 1885 (criminal); A Siberian "etape" or exile station house 1885; Common convicts on their way to Eastern Siberia. Lunch on the road near Tomsk, 1885; Exiles on their way to Siberia in the olden time. From a painting by V. Jacobi; Old house once occupied by "Decembrist" exiles. Irkutsk, 1885; Front of Alexandrofski central prison, Eastern Siberia (near Irkutsk) 1885; The Siberian prison of Irkutsk, Eastern Siberia, 1885; Room that Kennan and Frost occupied during their stay in Minusinsk; Prison in Tobol'sk, western Siberia, 1885; Prison in Tobol'sk, western Siberia, 1885; A part of Turukhansk on the Yenisei River, Eastern Siberia. Drawing by a political exile; River front at Tiumen showing exile barges, 1885; Tura river and steamboat landing at Tiumen showing steamers and convict barges 1885; Convict barge in the ice at Tiumen, 1885; Old Earth works. Containing Prison and Barracks. Ust Kamenagorsk; Type of house in which exiles live in province of Yakutsk, eastern Siberia. Original drawing by Vladimir Korolenko; An old prison in eastern Siberia (In the Upper Settlement at the Mines of Kara; Group of common convicts at the mines of Kara; Common convicts at work; mines of Kara, 1885; Potulov, Nikolai Andreyevich, governor of the Kara prisons; Trubetzkoi bastion. Peterpaulski fortress. Drawn by Clemens; Mines of Kara; Eastern Siberia. Prison, barracks in 1885; Mines of Kara; eastern Siberia; View of Lower Settlement; P. A. Kropotkin. Sculpture by Alexander Zeitlin; P. A. Kropotkin. Sculpture by Alexander Zeitlin; Peshekhonov, Aleksei 
Vasil'yevich, 1867-1933; Ivanchin-Pisarev, A. I.; Groups of photographs of Russian revolutionaries; Groups of photographs of Russian revolutionaries; Groups of photographs of Russian revolutionaries; Nathalie Golovine; Unidentified; Group of Social-Revolutionaries (O. S. Minor, V. L. Burtzev and others); Agapov, Semion Ivanovich, 1853-, Gernet. and 3 Poles (exiles of 1863); Mariya Spiridonova; Mariya Spiridonova.

\section{Kennan, George (1845-1924), compiler.}

European Russia; Portraits, Cities and Villages, Street Scenes and Country Views, Ethnic and Occupational Types. Album of Pictures Collected by George Kennan. [St. Petersburg, etc., 188-]. 1 v. 92 photographs and postcards with typed captions of sitters and locations, $33 \times 28.5 \mathrm{~cm}$. Mounted and bound by The New York Public Library, 1936.

Russia - Description and travel. Portraits, Russian. MFY+ 96-4041

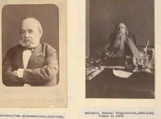
Alexander II, emperor of Russia, 1818-1881/Alexander III, emperor of Russia, 1845-1894; Future Nicholas II, emperor of Russia, 1868-1918/Trepov, Fiodor Fiodorovich, 1803-1889/ Tolstoi, Dmitrii Andreyevich, graf, 1823-1889, Minister of the Interior; Loris-Melikov, Mikhail Tarielovich, graf, 1825-1888/Pobedonostzev, Konstantin Petrovich, 1827-1907; Ignat'yev, Nikolai Pavlovich, graf, 18321908, Minister of the Interior/Bobrikov, Nikolai Ivanovich, 1839-1904; Sipyagin, Dmitrii Sergeyevich, 1853-1902, Minister of the Interior/[Postmortem photograph of an unidentified man]; Goncharov, Ivan Aleksandrovich, 1812-1891/Saltykov, Mikhail Yevgrafovich, 1826-1889 (taken in 1888); Saltykov, Mikhail Yevgrafovich, 1826-1889; Gorki, Maksim, pseud. of A. M. Peshkov, 1868-1936; Gorki, Maksim, pseud. of A. M. Peshkov, 1868-1936; Count Tolstoi as a guest in the home of his friend, V. G. Chertkov, in Meshchesrskoye. June 1910/Count Tolstoi riding horseback at Yasnaya Polyana. Feb. 1908; Lev Tolstoi and his family; Lev Tolstoi; [2 postcards of St. Petersburg monuments]; The Emperor's "Cottage", Alexandria Park, Peterhoff $[\ldots]$ /Entrance to the New Hermitage Picture Gallery, St. Petersburg; [postcard of the Winter Palace/postcard of the inside of Duma]; St. Petersburg/St. Petersburg (from Vail'yevski Ostrov, near $11^{\text {th }}$ line); The Lane, and the Old Palace, Tzarskoe Selo [...]; St. Petersburg. A Church/St. Petersburg. Ships on Neva; Artificial Ruins of Catherine II (Old Palace); St. Petersburg. Shipping in Neva/St. Petersburg. Fontanka; St. Petersburg. Petropavlovski fortress/St. Petersburg. Birzha; St. Petersburg. Barks on the Fontanka/St. Petersburg. Chernyshevski most over Fontanka; St. Petersburg. Kryukov Kanal/St. Petersburg. Kryukov canal; St. Petersburg. Neva. Live fish garden/St. Petersburg; Preeyoot Prince Oldenborgskaho. St. Petersburg/Tartar mosque, St. Petersburg Govt.; [Group of peasant children]/Lunch stall. St. Petersburg; Kolpino. St. Petersburg Govt./Village in Kolpino. St. Petersburg Govt.; Woods near [Riadovo], St. Petersburg Govt./ Village of [Riadovo]. St. Petersburg Govt.; Village of [Riadovo], 
St. Petersburg Govt.; Great Russian peasant/Haying: village of Kopov. St. Petersburg Govt.; Returning from harvesting. St. Petersburg Govt./Izba in St. Petersburg Govt.; Laborer resting. St. Petersburg/Great Russian muzhik; Moscow; Moscow. Panoramic view of the City (and Kokoreff Hotel)/ Moscow. Genera View of the Kremlin (taken from the wo[o]den brid[g]e); [Postcard of Moscow]/Moscow. S. W. corner of Kremlin; The Western end of the Kremlin - Moscow/Moscow, June 3, 1886. St. Basil Cathedral; Moscow. Church of St. Basil/Moscow. Church of St. Basil; Moscow, June 8, 1886. Great-bell at food of Tower nd Tower of Ivan the Terrible in Kremlin/ Moscow, June 8, 1886. Devichi Nunnery. (Nuns wearing high pointed caps and veils); Novgorod; [Boat]/Village on the Volga river; Wattled granary and farm buildings (Central and Southeast Russia. Volga Region); Rybinsk/ Rybinsk; [View of unidentified town]; [View of unidentified town with river]; Nizhni Novgorod from the River/[View of unidentified town]; Genera view of Nizhni from above/[View of unidentified town from a river]; [View of unidentified town]; [View of unidentified town]; Iz za ozera vid n staryi iarmorochnyi sobor/Kazanskii s'iezd; Nizhni Novgorod. View from Fair. River Oka in foreground. Kremlin in distant point; Nizhnii baraz. Tserkov' Il'I proroka. Il'inskii s'iezd, vdali iarmarka/[View of town from the river]; View of Nizhni Novgorod from the river Oka; [View of unidentified town]/Church in Nizhni Novgorod. Rozhdestvenskaya (Stroganovskaya) on the Lower Bazaar; Street in Nizhni Novgorod/Scene on the River at Nizhni Novgorod in Fair Time; Nizhni Novgorod. Iarmarka s grebeshka/Fair ground of Nizhni Novgorod; Nizhni Novgorod. Kitaiski Road/Nizhni Novgorod. Kitaiski Ryad; [Unidentified town]/ Kunavino. Nizhni Novgorod/[Unidentified town]; Wind mills. Simbirsk/ Village of Znamenka. Simbirsk; Tartar school. Simbirsk Govt./Krest'yanin. Knyazhi Gore, Simbirsk Govt.; Simbirsk Govt./Mordva. Simbirsk; Talk at the chasovnya (Mordva)/Mordva from Simbirski Govt. Taken at Kamenskoi fair; General view of a part of Perm; Perm Street. Perm/Iron works. Perm; Perm, from heights east of city. 1885; [Four photographs of priests]; Russian priest. St. Petersburg/[Two photographs of nuns]; Under officer (type) of time of Nicholas I/Turk. (One of the emperor's guards). St. Petersburg/[Three photographs of officers in uniform]; [Five photographs of types: peasant; soldier; seller]; [Five photographs of types: sellers and peasants]; [Man with two women]/[Man with two women]; [Woman in indigenous clothing]; Russian nurse. St. Petersburg/Russian nurse. St. Petersburg/[Three photographs of types]; Common peasant. St. Petersburg/Russian babka - old woman. St. Petersburg/ Church beggar. St. Petersburg/Nun begging at door of church. St. Petersburg/ Russian chimney sweep; Fruit seller. Gostini Dvor. St. Petersburg/Balalaika player. St. Petersburg/Russian coachman (private). St. Petersburg/Droshky and driver. St. Petersburg; [Carriage with two horses]/Retired kossacks; [Two photographs of Russian women dressed as peasants]; [Woman dressed as a peasant]/ 
Little Russian woman. (Ukrainian woman); The russification of Dorpat University. Photographed from the original drawing by Man von Oettingen, Berlin, and given to George Kennan in London Feb. 14, 1894 by his friend Poultney Bigelow; [A group of men]/[An old man]; Poltava Govt. Ukrainian types; [Photograph of a painting depicting peasants in the field]; Good bye by Baron Klodt; Mill dam and stream. Selo Berdskoe. Plotina mel'nitsy; Church in Chuvash settlement/[Church]; [Two photographs of peasant houses]; [A peasant house]/Buben' ... s v vnutrennei storony/[Peasant houses]; [Peasant houses]/ Tolkuchii rynok; Tolkuchi rynok/Tolkuchii rynok. Leather market; Tolkuchi rynok. Old clothes shop/Tolkuchi rynok. Household utensils stand; Tolkuchii rynok. Iron shop/[Chuch and fortress]; [Unidentified town]/[Photograph of a landscape painting]; [Landscape with a river]/[A church]/[An administrative building]/[Landscape with a river]; [General view of an unidentified town].

Khram vo imia Khrista Spasitelia v Moskvie.

Moscow: Sherer Nabgol'ts i Ko., 18-? 34 mounted photographs, $58 \times 47 \mathrm{~cm}$. Title from original portfolio. Three manuscripts that belong to first photograph are on leaves. Some photos have name of the architect, Konstantin Ton. Four reconstruction papers based on the description of the exterior of the Cathedral were drawn by Franklin Sciacca.

Master Negative: ${ }^{\star}$ ZZ-32071 Church architecture - Russia. Khram Khrista Spasitelia (Moscow, Russia).

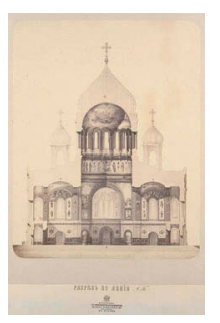

Slav. Reserve Photo (Khram vo imia Khrista Spasitelia v moskvie) SLV 063 [Cover title]; Razriez po linii A. B.; Detal' srednei chasti, vnutrennosti khrama; Detal' vnutrennosti, glavnago kupola khrama; Detal' lievoi storony, vnutrennosti khrama; Detal' pravoi storony, vnutrennosti khrama; Obshchii plan khrama, s mozaichnym polom; Obshchii vid vyemki zemli dlia fundamentov Khrama Voimia Khrista Spasitelia, fasad khrama i byvshago Aleksieevskago Monastyria (Glavnyi Arkhitektor Konstantin Ton. Risoval s natury N. Benua); Sposob zagotovleniia materialov dlia postroeniia v Moskvie Khrama Voimia Khrista Spasitelia (Glavnyi Arkhitektor Konstantin Ton. Risoval s natury A. Krakau); Raznye predmiety otnosiashchiesia k zakladkie Khrama Voimia Khrista Spasitelia sovershivsheisia 10 sentiabria 1839 g. (Glavnyi Arkhitektor Konstantin Ton. Risoval s natury A. Rezanov); Konstruktsiia glavy, glavnago kupola Khrama, vo imia Khrista Spasitelia; Fasad podkupol'noi glavnoi arki khrama so storony koridora, i plan podkupol'nago kol'tsa (Glavnyi Arkhitektor Konstantin Ton); Plan i razriez podkupol'nykh arok i parusov glavnago kupola khrama (Glavnyi Arkhitektor Konstantin Ton); Razriez glavnago kupola khrama (Glavnyi Arkhitektor Konstantin Ton); Oblitsovka mramorom naruzhnykh stien khrama (Glavnyi Arkhitektor Konstantin Ton); Fasad i razriez arok, pilonov, parusov i kol'tsa glavnago kupola khrama (Glavnyi Arkhitektor Konstantin Ton); Zapadnaia storona svoda glavnago kupola (Glavnyi Arkhitektor 
K. Ton. Zhvopis' Professora Markova); Barel'ef Moisei i Mariam (Sochinil i isponil Professor A. V. Logonovskii); Svod glavnago kupola khrama (vostochnaia storona) izobrazhenie gospoda Savaofa (Sochinil i pisal Professor A. T. Markov); Barel'ef David v sobranii vel'mozh, peredaet chertezhi khrama Solomonu (Sochinil i isponil Professor A. V. Logonovskii); Pravaia storona barel'efa iavlenie Arkhangela Mikhaila Iisusu Navinu (Sochinil i ispolnil Professor A. V. Logonovskii); Lievaia storona barel'efa iavlenie Arkhangela Mikhaila Iisusu Navinu (Sochinil i ispolnil Professor A. V. Logonovskii); Barel'ef David po pobiedie nad Goliafom srietaemyi likom zhen (Sochinil i isponil Professor A. V. Logonovskii); Vostochnaia storona svoda glavnago kupola (Glavnyi Arkhitektor K. Ton. Zhivopis' Professora Markova); Rieshetka na galerei poiasa glavnago kupola. (Glavnyi Arkhitektor K. Ton. Zhivopis' Professora P. Basina); Poias glavnago kupola (Glavnyi Arkhitektor K. Ton. Zhivopis' Professora P. Basina); Poias glavnago kupola (Glavnyi Arkhitektor K. Ton. Zhivopis' Professora P. Basina); Poias glavnago kupola (Glavnyi Arkhitektor K. Ton. Zhivopis' Professora P. Basina); Poias glavnago kupola (Glavnyi Arkhitektor K. Ton. Zhivopis' Professora P. Basina); Poias glavnago kupola (Glavnyi Arkhitektor K. Ton. Zhivopis' Professora P. Basina); Poias glavnago kupola (Glavnyi Arkhitektor K. Ton. Zhivopis' Professora P. Basina); Poias glavnago kupola (Glavnyi Arkhitektor K. Ton. Zhivopis' Professora P. Basina); Poias glavnago kupola (Glavnyi Arkhitektor K. Ton. Zhivopis' Professora P. Basina); Miestnyi obraz v ikonostasie glavnago pridiela Khrama voimia Khrista Spasitelia Iisus Khristos (Pisal Professor Nef); Miestnyi obraz v ikonostasie glavnago pridiela Khrama voimia Khrista Spasitelia rozhdestvo Khristovo (Pisal Professor Nef).

[Khram vo imia Khrista Spasitelia v Moskvie] Moskva: Shere Nabgol'ts i Ko., 188-? 54 mounted photographs, 59 x 45cm. Title from original portfolio.

Church architecture - Russia. Khram Khrista Spasitelia (Moscow, Russia).

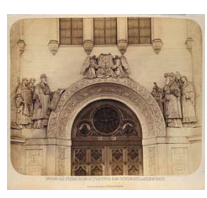
Slav. Reserve (Photo) (Khram vo imia Khrista Spasitelia) SLV 064 [Cover title]; [Cathedral of Christ the Savior]; Vid Khrama vo imia Khrista Spasitelia iz sada dvortsa Aleksandrii; Barel'ef nad srednimi vratami vostochnoi storony khrama sviatiteli Petr i Aleksii Moskovskie. Sochinen i ispolnen P. K. Baronom Klotom; Barel'ef nad srednimi vratami sievernoi storony Khrama S. S. Apostoly Petr i Pavel. Sochinen i ispolnen Professorom A. V. Loganovskim; Barel'ef nad srednimi vratami zapadnoi storony khrama izobrazhaiushchii angelov (1812 god.) Sochinen i ispolnen Professorom A. V. Loganovskim; Barel'ef nad pravymi vratami zapadnoi storony khrama izobrazhaiushchii angelov s khorugviami (opolchenie 1812 goda.) Sochinen i ispolnen Professorom A. V. Loganovskim; Barel'ef nad lievymi vratami zapadnoi storony khrama izobrazhaiushchii angelov so znamenami (1812 god.); Sochinen i ispolnen Professorom A. V. Loganovskim; Barel'ef nad pravymi vratami vostochnoi storony Khrama Sviatiteli Iona i Filipp 
Moskovskie. Sochinen i ispolnen P. K. Baronom Klotom; Barel'ef nad lievymi vratami vostochnoi storony khrama S. S. Stefan Permskii i Prepodobnyi Sergii. Sochinen i ispolnen P. K. Baronom Klotom; Barel'ef Prepodobnyi Sergii blagoslovliaet na bran' V. K. Dmitriia Donskago i daet emu peresvieta i osliabia. Sochinen i ispolnen Professorom A. V. Loganovskim; Barel'ef Avraam s soiuznikami vozvrashchaiushchiisia poslie pobiedy nad tsariami i vstriechaemyi Mel'khisedskom. Sochinen i ispolnen Professorom A. V. Loganovskim; Barel'ef rozhdestvo Khristovo i poklonenie pastyrei. Sochinen i ispolnen Professorom A. V. Loganovskim; Barel'ef Prepodobnyi Dionisii blagoslovliaet Kniazia Pozharskago i grazhdanina Minina na osvobozhdenie Moskvy. Sochinen i ispolnen Professorom A. V. Loganovskim; [Inside main cupola]; [Inside main cupola]; [Inside main cupola]; [Inside main cupola]; [Inside main cupola]; [Inside main cupola]; [Inside main cupola]; Poias gravnago kupola. Zhivopis' N. A. Kosheleva; Poias gravnago kupola. Zhivopis' N. A. Kosheleva; Vid glavnago oltaria; Izobrazhenie "Slovo Plot' Byst" napisannoe profes. Koshelevym v svodie Iuzhnago Kryla khrama; Preobrazhenie Gospodne; Soshestvie Sviatago Dukha; Voskresenie Khristovo; Voznesenie Gospodne; Sviatoi Apostol i Evangelist Ioan Bogoslov; Sviatoi Apostol i Evangelist Luka; Izobrazhenie Dievo dnes' Presushchestvennago rozhdaet (Kondak), napisannoe profes. Vereshchaginym na vostochnoi stienie glavnago Altaria khrama; Soshestvie Sv: Dukha (Pis. Pr. Nef); Srietenie (Pis. Pr. Nef); Sv: Troitsa (Pis. Pr. Nef); Vvedenie vo khram (Pis. Pr. Nef); Kreshchenie (Pis. Pr. Nef); Vozdvizhenie kresta (Pis. Pr. Nef); Rozhdestvo Bogoroditsy (Pis. Pr. Bronnikov); Rozhdestvo Bogoroditsy (Pis. Pr. Bronnikov); Vvedenie vo khram (Pis. Pr. Bronnikov); Uspenie Bogoroditsy (Pis. Pr. Bronnikov); Blagovieshchenie (Pis. Pr. Bronnikov); Glavnyi oltar'. Sniatie so Kresta (Prof. V. P. Vereshchagina); Gornee miesto, Tainaia vecheria (Prof. G. I. Semiradskago); Sievero-Zapadnaia nisha, Blagoslovenie Sv. Sergiem Dmitriia Donskago (Prof. V. P. Vereshchagina); SieveroZapadnyi parus Evangelist Matviei (Prof. E. S. Sorokina); Iugo-Zapadnyi parus, Evangelist Mark (Prof. E. S. Sorokina); Iugo-Vostochnaia nisha, Poklonenie volkhvov (Prof. V. P. Vereshchagina); Sievero-Vostochnaia nisha, Poklonenie pastyrei (Prof. V. P. Vereshchagina); Iugo-Zapadnaia nisha, Pomazanie Davida na tsarstvo Samuilom (Prof. V. P. Vereshchagina); Zapadnaia storona; Iuzhnaia stiena Zapadnago kryla; Iuzhnaia storona; Sievernaia storona.

Kievskii Vladimirskii sobor. Kiev: Fotografiia G. G. Lazovskago, [1896?] 84 mounted photographs in 3 volumes, $70 \times 53 \mathrm{~cm}$.

Mostly photographs of the murals in the Cathedral of St. Vladimir (Volodymyr), Kiev, executed by V. N. Vasnetsov, M. V. Nesterov, A. V. Prakhov, P. A. Svedomskii and V. A. Kotarvinskii. Mounted albumen photographs. 
From Iaroslav D. Isajevych: Three volumes. The presentation album to Grand Duke Konstantin Konstantinovich (1858-1915) contains many photographs taken at the Kiev studio of G. G. Lazovskii shortly after the consecration of the cathedral. These are excellent reproductions of murals, icons, sculpted panels, as well as furniture and church utensils executed for the cathedral. There are also photographs of general views of the cathedral and of its interiors.

Photographs are extremely important since many murals were damaged and some church utensils have been lost or destroyed. In many cases, photographs from this album provide the only reliable source of information on the unique works of Orthodox religious art. All three volumes have original leather bindings with identical gilded inscriptions on the upper cover of each volume, namely: "Ego Imperatorskomu Vysochestvu Gosudariu Velikomu Kniaziu Konstantinu Konstantinovichu." The page and captions are printed (text in Russian). Albums previously belonged to the Library of the Academy of Historical Material Culture (Biblioteka Akademii Istorii Material'noi Kul'tury).

Metropolitan New York Library Council, 2008. Slavic Recon Project. Lazovskii, G. G. Cathedrals - Ukraine - Kiev.

Mural painting and decoration-Ukraine-Kiev. Cathedrals - Ukraine Kiev.

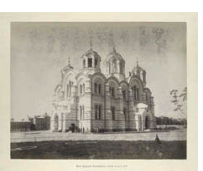

Slav. Reserve (Photo) (Kievskii Vladimirskii sobor) SLV 065 I: [Cover title with 2 images of presentation medals]; Bogomater' v absidie Glavnago Altaria; Glavnyi altar'. Tsentral'naia chast' Evkharistii; Glavnyi altar'. Pravaia storona Evkharistii. Arkhangel Gavriil; Glavnyi altar'. Arkhangel Mikhail. Lievaia storona Evkharistii; Glavnyi altar'. Proroki: Daniil, Moisei, Isaiia, Avvakum, Iezekiil, Patriarkh Iakov; Glavnyi altar'. Sievernaia storona. Proroki: Gedeon, Iliia, Ieremiia, Naum, Tsar' Solomon, Tsar' David; Glavnyi altar'. Iuzhnaia storona. Sv. Ottsy Rossiskoi tserkvi. Sv. Sergii Radonezhskii. Sv. Stefan Velikopermskii. Sv. Antonii Pecherskii. Sv. Feodosii Pecherskii. Sv. Aleksii Moskovskii. Sv. Petr Moskovskii; Glavnyi altar'. Sievernaia storona. Sv. Ottsy Vselenskoi tserkvi. Afanasii Velikii. Papa Rimskii Kliment. Vasilii Velikii. Grigorii Bogoslov. Ioann Zlatoust. Nikolai Murlukiiskii; Miestnye obraza ikonostasa Glavnago Altaria; Ikonostas Glavnago Altaria. Sv. Ravnoapostol'nyi Kn. Vladimir. Sv. Kniaginia Ol'ga; Ikonostas Glavnago Altaria. Sv. Mariia Magdalina. Sv. Kn. Aleksandr Nevskii; Ikony Glavnykh Tsarskikh Vrat; Sv. Ap. Pavel. Srietenie Gospodne. Sv. Ap. Andrei Pervozvannyi; Sv. Much. Kn. Mikhail Chernigovskii. Sv. Much. Kn. Boris. Sv. Much. Kn. Glieb. Sv. Much. Kn. Mikhail Tverskoi; Sv. Prep. Nestor Lietopisets. Sv. Kniaginia Evdokiia; Mnogoochivyi shestikrylyi Kheruvim. Sv. Kn. Evfrosiniia Polotskaia. Sv. Prep. Alipii zhvopisets pecherskii. Mnogoochvyi shestikrylyi Kheruvim; Sv. Kn. Andrei Bogoliubskii. Sviatoi Prepodobnyi Kuksha. Sv. Nikita Episkop 
Novgorodskii. Sv. Prokopii Ustiuzhskii o Khristie Iurodivyi; Iisus Khristos v glavnom kupolie; Kol'tso pod oknami glavnago kupola. Preddverie raia; Potolki glavnago prodol'nago korablia. Skorb’ Boga Otsa pri vidie Raspiatago Syna ("Otechestvie"); Potolok nad zapadnymi khorami. Iisus Khristos Slovo Bozhie. Potolok nad prestolom v Glavnom Altarie; Zapadnykh khor sievernaia storona. Blazhennoe sostoianie pervykh cheloviekov; Zapadnykh khor iuzhnaia storona. Iskushenie; Strashnyi sud; Kreshchenie Kn. Vladimira; Kreshchenie Rusi; Plashchanitsa; Parusa glavnago kvadrata. Sv. Evangelist Ioann. Sv. Evangelist Luka;

II: Parusa glavnago kvadrata. Sv. Evangelist Matfei. Sv. Evangelist Mark; Sv. Solunskie brat'ia. Sv. Mefodii. Sv. Kirill; Ikonostas zhertvennika glavnago Altaria. Sv. Tsaritsa Elena. Sv. Tsar' Konstantin; Ikonostas Diakonika glavnago Altaria. Sv. Arsenii Velikii. Sv. Nikolai chudotvorets Murlukiiskii; Ikonostas Diakonika glavnago Altaria. Sv. Velikomuchenitsa Varvara. Sv. Filaret Milostivyi; Zaprestol'nyi obraz v pridielie Sv. Kn. Ol'gi. Rozhdestvo Khristovo; Bogoiavlenie; Zaprestol'nyi obraz v sievernom pridielie na khorakh. Voskresenie Khristovo; Ikonostas sievernago pridiela na khorakh. Sv. Muchenik Kn. Boris. Bozhiia Mater'. Sv. Muchenik Kn. Glieb; Ikonostas iuzhnago pridiela na khorakh. Sv. Mitropolit Mikhail. Iisus Khristos. Sv. Kn. Ol'ga; Miestnyia ikony iuzhnago prediela na khorakh; Obraza dlia dvukh Tsarskikh Vrat na khorakh. Sv. Muchenikov Kn. Borisa i Glieba. Sv. Kn. Ol'gi; Voskresenie Lazaria; Viezd v Ierusalim; Tainaia Vecheria; Molitva v Gefsimanskom sadu; Sud Pilata; Golgofa; Potolok na sievernykh khorakh. Voznesenie Gospodne; Potolok nad iuzhnymi khorami. Sotvorenie mira. Pervyi den' tvoreniia; Potolki nad iuzhnymi khorami. Vtoroi den' tvoreniia. Sotvorebue mira. Tretii den' tvoreniia; Sv. Kn. Evfrosiniia Suzdal'skaia. Sv. Kn. Petr Muromskii. Sv. Kn. Fevroniia Muromskaia. Sv. Kn. Dovmont Gavriil Pskovskoi; Sv. Prep. Kn. Feodor Ostrozhskii. Sv. Gurii Arkhiepiskop Kazanskii. Sv. Innokentii episkop, chudotvorets Irkutskii. Sv. Prep. Mikhail iurodivyi, chudotvorets Novgorodskii; Bog-Otets opochivshii ot vsiekh diel svoikh. Potolok v zhertvennikie glavnago altaria. Dukh Bozhii, nosiashchiisia vverkhu vody. Potolok v diakonnikie glavnago altaria; Potoloki pod sievernymi khorami. Chetvertyi den' tvorenia. Sotvorenie Mira. Piatyi den' tvoreniia; Potolok pod sievernymi khorami. Sotvorenie Mira. Shestoi den' tvoreniia; Potolok na iuzhnykh khorakh. Preobrazhenie Gospodne; Potolok na khorakh. Kupol na khorakh;

III. Zhivopis' na kolonnakh khor: Sv. Nifont, episkop Novgorodskii. Sv. Filipp, mitropolit Moskovskii. Sv. Pafnutii Borovskii. Sv. Mitrofan, episkop Voronezhskii; Zhivopis' na kolonnakh khor: Sv. Iosif Vologotskii. Sv. Nikita Stolpnik. Sv. Varlaam Khutynskii. Sv. kn. Feodor Chernyi; Sv. Nikola Sviatosha Kn. Chernigovskii. Sv. Kniaginia Irina. Sv. Kn. Vladimir Iaroslavich. Sv. Avraamii Smolenskii; Vid Kievskago Vladimirskago sobora s zapadnoi storony; Vid Kievskago Vladimirskago sobora s iu.-z. ugla; Sv. vel. kn. Ol'ga. Sv. vel. kn. 
Vladimir; Kievskii Vladimirskii sobor. Vid ot zapadnykh vrat na Glavnyi Altar'; Kievskii Vladimirskii sobor. Vid na iuzhnuiu storonu glavnago prodol'nago korablia; Kievskii Vladimirskii sobor. Vid na sievernuiu storonu glavnago prodol'nago korablia; Obshchii vid na zapadnyia vrata. Mramor, bronza, zhivopis' na steklie A.V. Prakhova. Stienopis' V. M. Vasnetsova; Ikonostas i klirosy Ol'ginskago pridiela na iu. khorakh; Vid na sievernye khory ot Borisogliebskago pridiela. Ornamentnoe rospisanie ispolneno po eskizam A. S. Mamontova; Mramornaia balliustrada zapadnykh khor; Mramornyia perila s inkrustatsiei na iu. i s. khorakh; Oblitsovka vkhoda na glavnuiu liestnitsu; Bronzovyi zolochenyi emal'irovannyi kiot, ispolnennyi na fabrikie $t$-va A. M. Postnikova v Moskvie. Obraz Sv. Nikolaia Murlikiiskago raboty N. K. Pimonenko; Glavnyia Tsarskiia Vrata iz zolochenoi bronzy. Ispolneny na fabrikie t-va A. M. Postnikova v Moskvie. Zhvopis' V. M. Vasnetsova; Obshchii vid na Glavnyi Prestol; Zhertvennik; Bronzovaia, zolochenaia, s emal'irovannymi tsvietami, rieshetka na soleie Glavnago Ikonostasa. Ispolnena na fabrikie t-va A. M. Postnikova v Moskvie; Khorugv' iz zolochenoi emal'irovannoi bronzy, prinesennaia v dar Kievskomu Vladimirskomu soboru Nizhnim-Novgorodom; Malaia khorugv' v pridielie na khorakh s obrazom Sv. Kn. Vladimira; Bronzovaia, zolochenaia khorugv's obrazom Sv. Kn. Ol'gi na litsevoi storonie i Nerukotvorennym obrazom na obratnoi storonie; Semisvieshchnik i tri podsviechnika iz zolochenoi bronzy. Ispolneny na fabrikie t-va A. M. Postnikova v Moskvie; Piatisvieshchnik, podsviechnik k miestnym obrazam glavnago ikonostasa i analoi iz zolochenoi, emal'irovannoi bronzy. Ispolneny na fabrikie t-va A. M. Postnikova v Moskvie; Panikadilo (khoros) vtoroi velichiny iz zolochenoi bronzy. Ispolneno na fabrikie $t$-va A. M. Postnikova v Moskvie; Protoiereiskoe kreslo, stul, taburet i skladnoi analoi iz duba i oriekha; Lar' iz duba i oriekha s pozolotoi.

Krasnoe Selo. [St. Petersburg: Khudozh. fototip. A. I. Vil'borg, n.d.].

28 leaves of plates: all ill.; $17 \times 25 \mathrm{~cm}$. Photographs by N. Digo. "Fotograf Voisk Gvardii N. Digo." "Perl. Jun 18, 1931."

Metropolitan New York Library Council, 2008. Slavic Recon Project. Krasnoselskoe (Leningradskaia oblast, Russia) - Pictorial works.

Slav. Reserve (Photo) (Krasnoe selo) SLV 066 Vid na ozero, Dudergof i Kavelakhtu; Gauptvakhta; Strielka na dorogie v Striel'nu i Pavlovskuiu Slobodu; Konoviaz'; Pamiatnik Imperatoru Aleksandru II; Ofitserskoe sobranie L.-G. Konnago Polka; Ofitserskoe Sobranie Kavalergardskago, Eia Velichestva, polka; Pravyi flang Glavnago lageria; Shtabnyi dom avangardnago lageria; Krasnosel'skaia tserkov'; Tsarskaia Stolovaia; Palatki dlia bol'nykh, v sadu gospitalia; S Kavelakhty na avangardnyi lager', ozero i Krasnoe Selo; S Kavelakhty na Dudergof, ozero i Krasnoe Selo; Voennyi gospital'; Pravyi flang avangardnago lageria; Dvorets Ego Imperatorskago Velichestva; Teatr; Dvorets E. I. V. Velikago Kniazia Vladimira Aleksandrovicha; Glavnyi v'ezd 
v Krasnoe Selo; Pokhodnaia tserkov'; Dvorets E. I. V. Velikago Kniazia Mikhaila Nikolaevicha; Pavlovskaia Sloboda, raspolozhenie Kavalergardskago, Eia Velichestva, polka; Stantsiia Krasnoe Selo. Baltiiskoi zhel. dor.; Ot signal'noi pushki na teatr i Dudergof; Novaia tserkov' v glavnom lagerie, u L.-G. Semenovskago polka; Shtab voisk lagernago sbora; Gospital' i novaia Kalancha, na pravom flangie avangardnago lageria.

Krestovskii, Vs. Vl. (Vsevolod Vladimirovich), 1840-1895. KatselevoAblavskii boi, 24 avgusta 1877. [Moscow: s.n., 1882?] [465]-502 p.: port.; $21 \times 13.5 \mathrm{~cm}$.

Text signed: Vsevolod Krestovskii. Originally published in Russkii viestnik 1882, v. 161. One photograph mounted on page facing p. [465], signed A. Iasvoin. Includes bibliographical references. "Perl. Jun 18, 1931."

Custom binding. On cover: Komandiru 12-go Arm. Korpusa Ego Imperatorskomu Vysochestvu Velikomu Kniaziu Vladimiru Aleksandrovichu.

Metropolitan New York Library Council, 2008. Slavic Recon Project. Russo-Turkish War, 1877-1878. Russkii viestnik.

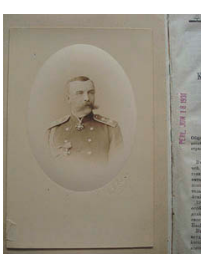

Slav. Reserve (Photo) (Krestovskii) SLV 067 [A portrait of Vsevolod Vladimirovich Krestovskii].

[Krym: an album of photographs]. [S. 1.: s. n., 1885?] 54 mounted photographs, $35 \times 52 \mathrm{~cm}$. Front cover has an embossed imperial crown and initials of A. M. [Grand Duke Aleksandr Mikhailovich, 1866-1933] and the word "Krym". One half black leather, blue silk cloth, white moiré endpapers. Gilt title on spine. Inscribed in pencil on front free endpaper: "George of Russia, presented by Mr. Perlstein, March 19th, 1937, New York.”

Konstantin Collection. Crimea (Ukraine). Crimea (Russian Federation). Photograph albums-Russia-Specimens.

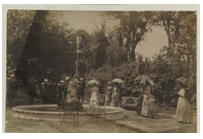

Slav. Reserve (Photo) 05-2969 SLV 068 [Alexander III and Grand Dukes]; [Alexander III and Grand Dukes]; [Ladies, officers, and sailors near a fountain, "Venera"]; [Alexander III and royal family]; [Alexander III and Grand Dukes]; [Kabardians at Old Palace]; [Alexander III talking with Kabardians at Old Palace]; [Alexander III and Grand Dukes]; [Grand Duke Aleksandr Mikhailovich]; [Officers and soldier at a stop]; [View of a village in the mountains]; [General view of Yalta]; [General view of Yalta]; [People in national dresses]; [Three women and a girl in national dresses]; [Local people]; [Palace]; [Local people]; [Local people]; [Palace]; [A woman in national dress]; [A woman in national dress]; [A man in front of a palace]; [Park]; [Grand Dukes]; [A woman in front of a house]; [Children in front of a house]; [Seaside]; [Seaside]; [Local people]; [Local people]; [Seaside]; [View of the sea]; [Local people]; [Seaside]; [Grand Duke with a child]; [Children]; [Alexander III at a railroad station]; [Alexander III at a railroad station]; [A group of officers]; [People in a room]; [A group of military and civilian 
people]; [A group of military and civilian people]; [A group of men with daggers]; [A group of men in a sleigh]; [A group of military and civilian people in a sleigh]; [Large group of people]; [Officers/Officers]; [A palace]; [A palace]; [Officers hunting]; [Officers hunting]; [Children with a teacher].

Materialy dlia istorii Imperatorskago Moskovskago vospitatel'nago doma. Moscow: Tipografiia Semena, 1863. iv, 113 p., 4 plates: ill., ports.; $34 \times 28 \mathrm{~cm}$. "Vypusk 1-i, izdannyi ko dniu stolietniago iubileia Vospitatel'nago Doma, 1 sentiabria 1863 g." Prefatory article signed: V. Drashusov. Printed in double columns.

Six mounted photographs. Bookplate of Grand Duke Vladimir Aleksandrovich. Imperatorskii Drashusov, V. Moskovskii vospitatelnyi dom-History-Sources.

Maternity homes-Russia-Moscow-History- Sources. Orphanages-Russia (Federation)-Moscow History-Sources.

Maternity homes-Russia (Federation) - Moscow-History-Sources.

Slav. Reserve (Photo) 97-2595 SLV 072 Vid Vospitatel'nago Doma ot Moskvorietskago mosta; Moskovskii Vospitatel'nyi dom so storony bol'shago pod'iezda; Tserkov' Moskovskago Vospitatel'nago Doma; Bol'shoi korridor v okruzhnom stroenii.

Mezer, François de. Pamiat' 900-lietiia kreshcheniia Rusi/fotografiia deMezer. Kiev: Fr. de Mezer, 1888. 1 album, 5 photographs, $51 \times 44 \mathrm{~cm}$.

Cover title. "Eia Velichestvu Korolevie Ellinovie." Photographs mounted on card with gilt borders. With photographer's blindstamp (de Mezer/Kieff) on each print and lithograph label on verso. Original publisher's portfolio of blindstamped blue cloth with Imperial arms in gold on upper cover. Presentation copy to Olga Konstantinovna, Queen of Greece and sister of "K.R." Purchased from Bernard J Shapero Rare Books, 2007.

Photography-Ukraine-19th century. Photography-Russia-19th century. Kiev (Ukraine)-Pictorial works. Olga, Queen, consort of George I, King of the Hellenes, 1851-1926.

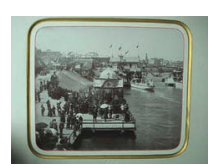

Slav. Reserve (Photo) 07-6535 SLV 073 [St. Vladimir Monument in Kiev]; [Commemorative celebration of the Christianization of Russia]; [Commemorative celebration of the Christianization of Russia]; [Steamboat 'Kniaz' Vladimir

Sviatoi"]; [Unknown monument].

\section{Moskovskii Publichnyi i Rumiantsevskii muzei.}

Moscow, 186-? 43 photographs on 24 leaves, 65 x $48 \mathrm{~cm}$. Mitreiter (Photographer). Cover title. In contemporary portfolio of purple velvet, white satin doublure, with title stamped in gold on cover. Microfilm. New York: New York Public Library, 1990. 1 microfilm reel; 35mm. (MN $\left.{ }^{*} Z Z-30641\right)$. Gosudarstvennyi Rumiantsovskii muzei. Museums - Russia - Moscow. 
Slav. Reserve (Photo) (Gosudarstvennyi Rumiantsovskii muzei) SLV 074 [Photograph of a drawing of Alexander II]; [Photograph of a drawing of Nicholas I]; Gosudarstvennyi Kantsler Graf N. P. Rumiantsev; Fasad Rumiantsevskago muzeia; Biblioteka Rumiantsevskago muzeia; Chital'nyi zal; Biblioteka Gosudaryni Imperatritsy Aleksandry Feodorovny; Kartinnyia gallerei, russkaia shkola; Kartinnyia gallerei, russkaia shkola; Khristos vo grobie, s kartiny Briulova; Tainaia vecheria, s kartiny Kramskago iz kartinnoi gallerei Moskovskago Publichnago muzeia; Statuia Grafa Zadunaiskago; Statuia mira (rabota Kanova); Otdielenie russkikh drevnostei. Raskopka Gr. A. S. Uvarova v kurganie na Iur'evoi gorie 1852 goda; Otdielenie russkikh drevnostei. Kostianoi rieznoi naloi Tsaria Aleksieia Mikhailovicha; Otdielenie do istoricheskikh drevnostei. 1) Kamennyi viek Rossii. 2) Bronzovyi viek. 3) Zhelieznyi viek; Persidskiia monety; Otdielenie rukopisei. I. Iz grecheskago paremeinika IX v. II. Iz latinskoi sluzhby Sv. Villigizu XII v. III. Iz sobstvennoruchnoi rukopisi Dziurdano Bruno. IV. Iz latinskago molitvoslova XIVXV v.; Otdielenie rukopisei. V. Iz Glagolicheskago Evangeliia XI v. VI. Bolgarskago paremeinika XII v. VII. Iz slaviano-russkago Evangeliia 1092 g. VIII. Iz slaviano-russkago Evangeliia 1164 g.; Otdielenie rukopisei. IX. Zaglavnyi list slav. staropech. izdaniia, L'vov, 1591 g. (unis). X. Zaglavnyi list slav. staropech. izdaniia, Kiev, 1618 g. (unis);

Zal Dashkovskago etnograficheskago muzeia; Dashkovskii etnograficheskii otdiel. Velikorussy. Bielorussy. Bolgary iz Makedonii. Gvebry (ognepoklonniki); Dashkovskii etnograficheskii otdiel. Poltavka. Liublinskaia Guberniia. Russkie v Pol'shie. Velikorussy; Dashkovskii etnograficheskii otdiel. Permiaki. Mordva. Kirgizy Orenburgskiia. Cherkes; Dashkovskoe sobranie izobrazhenii russkikh dieiatelei.

Novogeorgievsk. 1884. [St. Petersburg?]: Voennaia fotografiia Sh. K. Topornina, 1884. 17 mounted photographs (some folded), $31 \times 42 \mathrm{~cm}$. Each plate bears the mark of "Voennaia fotografiia Sh. K. Topornin" on verso. Contains two panoramic views (plates 16 and 17). "Perl. Jun 18, 1931."

Metropolitan New York Library Council, 2008. Slavic Recon Project. Topornin, Sh. K. Modlin (Poland). Modlin (Poland) -Buildings, structures, etc.

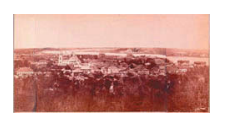

Slav. Reserve (Photo) (Novogeorgievsk) SLV 076 Konstantinovskiia vorota; Denovskaia bashnia; Sobor Sviatago Georgiia; Vodoprovodnoe zdanie; Gorzha Ravelina fronta Kniazia Varshavskago; Front Kniazia Varshavskago; Pontonnaia navodka; Provolochnyi most; Zhelieznodorozhnyi most; Ostrolenskiia vorota; Mikhailovskiia vorota; Kazematy pod valgangom; Vodokachal'nia; Parizhskii front; Ravelin Parizhskago fronta; Pravyi bereg Visly; Lievyi bereg Visly pri vpadenii Nareva. 
Ocherki Tverskoi stariny. Gorod Torzhok. 11 mounted photographs (some folded). $32 \times 40 \mathrm{~cm}$. Bound in purple plush with heavy gold, silver and white silk embroidery. With book plate of Grand Duke Vladimir Aleksandrovich. "Perl. Jun 18, 1931" Torzhok (Russia). Monasteries Russia (Federation) - Torzhok.

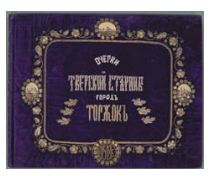

Slav. Reserve (Photo) (Ocherki Tverskoi stariny) SLV 077 [Binder's title; photograph of the hand-sewn cover]; Borisogliebskaia storona v Torzhkie: Borisogliebskii monastyr' i sobornyi khram Sv. Spasa. Tserkvi Sv. Georgiia Znamenskaia Uspenskaia Klimentovskaia i Kozmodem'ianskaia. Edinoviercheskaia tserkov'. Tserkov' Mironositskaia. Tserkov' dalnei troitsy i Nikolo pustynskaia tserkov'; Vid lievoi Zatveretskoi storony v Torzhkie: Chast' Borisogliebskoi storony. Voskresenskii dievichii monastyr'. Vozdvizhenskaia I Nikol'skaia tserkvi. Dmitrovskaia I Vasil'evskaia tserkvi. Vlas'evskaia Tser'kov'; Vvedenskaia tserkov' i khram Sv. Ioanna Bogoslova v Borisogliebskom monastyrie; Drevniaia Voznesenskaia dereviannaia tser'kov' v Torzhkie; Voskresenskii dievichii monastyr'; Putevoi dvorets v Torzhkie; Drevnii Obraz Uspeniia Presviatoi Bogoroditsy; Drevnee izobrazhenie Sv. Prep. Efrema; [Crucifixion]; Tip Novotorzhskoi kupchikhi v starinnom nariadie; Tip Novotorzhskoi kupchikhi.

[Odessa: an album]. [Russia?: s.n., 1900-1909?] 23 mounted plates, $14 \times 22 \mathrm{~cm}$.

Views showing monuments, public buildings, and other places of interest in Odessa. “May 29, 1941.” Metropolitan New York Library Council, 2008.

Slavic Recon Project. Odessa (Ukraine) - Pictorial works. MonumentsUkraine - Odessa - Pictorial works.

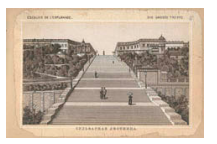

Slav. Reserve (Photo) (Odessa. an album) SLV 078 Vid s moria; Sobor; Pamiatnik Gertsogu Rishel'e; [Harbor]; Bul'varnaia Liestnitsa; Gavan'; Birzha; Rishel'evskaia ulitsa i teatr; Vokzal; Ekaterininskaia ulitsa; Rimsko-katolicheskaia tserkov; Ekaterininskaia ploshchad'; Pamiatnik Grafu M. Vorontsovu; Malen'kii fontan; Andreevskii liman; [Nicholas boulevard]; Nikolaevskii bul'var; Voennyi spusk; Panteleimonskoe podvor'e; Pamiatnik Imperatoru Aleksandru II; Universitet; Gorodskoi teatr; Skala Monakh na malom fontanie.

Olonetskaia guberniia. [Russia?: s.n., 188-?].

36 mounted photographs, $28 \times 37 \mathrm{~cm}$. Plates bear the stamp of Ia. Pekarskii. "Apr. 20 1934."

Inserted is a list, in manuscript, of 37 photographs; the first one is missing. Includes seven panorama views. Pekarskii, Ia. Olonets (Russia). Petrozavodsk (Russia).

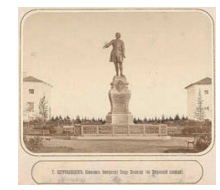

Slav. Reserve (Photo) (Olonetskaia guberniia) SLV 079 [Cover title]; G. Petrozavodsk: Pamiatnik Imperatoru Petru Velikomu (na Petrovskoi ploshchadi); G. Petrozavodsk: Gubernskiia prisutstvennyia miesta; G. Petrozavodsk: Gubernatorskii dom; 
Chuchelo medviedia, ubitago v Kargopol'skom uiezdie, v 1877 godu i prinadlezhashchago Olonetskomu gubernatoru Grigor'evu; G. Petrozavodsk: Zalo v gubernatorskom domie; G. Petrozavodsk: Terrassa i sad pri gubernatorskom domie; G. Petrozavodsk: Aleksandrovskii pushechno-snariadnyi zavod; G. Petrozavodsk: Chugunnyi portik na dvorie Aleksandrovskago zavoda, ustroennyi v pamiat' posieshcheniia Imperatora Aleksandra I-go v 1819 g.; G. Petrozavodsk: vid u gorodskoi tiur'my k sieveru: 1) Ovrag riechki Neglinki, 2) Kazarmy gubernskago bataliona, 3) Pogost Solomennoe na Sievernom kontsie zaliva, 4) Ekaterininskaia kladbishchenskaia tserkov' na beregu r. Neglinki, 5) Zemskaia bol'nitsa, 6) Petrozavodskii zaliv Onezhskago ozera [panorama]; G. Petrozavodsk: 1) Dom dukhovnoi seminarii (postr. v 1872 godu), 2) Dom gorodskago uchilishcha, 3) Dom gornago nachal'nika Olonetskikh zavodov; G. Petrozavodsk: Liuteranskaia tserkov'; G. Petrozavodsk: Dom, v kotorom zhil Olonetskii gubernator i pravitel' namiestnichestva Derzhavin (v 1784-1785 gg.); G. Petrozavodsk: 1) Obshchestvennaia pristan', 2) Spasatel'naia stantsiia, otkrytaia 1-go oktiabria 1875 goda, 3) Petropavlovskii sobor, postroennyi Imperatorom Petrom Velikim, v 1702 godu, 4) Khristorozhdestvenskii sobor, postroennyi v 1800 godu, 5) Sviatodukhovskii sobor, postroennyi v 1872 g.; G. Petrozavodsk: 1) Angliiskaia ulitsa, 2) Tri doma gornago viedomstva, 3) Dom gorodskago obshchestva, v kotorom pomieshchaetsia gorodskaia uprava, sobranie gorodskoi dumy i gorodskoe politseiskoe upravlenie, 4) Gostinnyi dvor, 5) Doma gubernskoi gimnazii, 6) Sviatodukhovskii Khristorozhdestvenskii i Petropavlovskii sobory; Pogost Solomennoe (v 6-ti verstakh ot g. Petrozavodska), Miestorozhdenie Solomennoi brekchii, 1) Liesopil'nyi zavod kommertsii sovietnika Gromova, 2) Starinnaia tserkov' byvshei Solomennoi pustyni [panorama]; Sloboda Konchezero (v 45-ti ver. ot g. Petrozavodska); Ozero Konchezero - vid iz sada upravitelia chugunno-plavilennago zavoda; Tserkov' Konchezerskago prikhoda i Chugunnoplavilennyi zavod v slobodie Konchezerie [panorama]; Vodopad Kivach; Vodopad Kivach, na riekie Sunie, v 70-ti ver. ot g. Petrozavodska. Obshchii vid na vodopad; Vodopad Kivach, na riekie Sunie, v 70-ti ver. ot g. Petrozavodska. Obshchii vid na vodopad; Vodopad Kivach, na riekie Sunie, v 70-ti ver. ot g. Petrozavodska: Vid s pavil'ona u samago vodopada; Rieka Suna, s vodapodom Kivach. Pavil'on na lievom beregu [panorama]; Konchezerskiia Martsial'nyia Vody, kotorymi pol'zovalsia Imperator Petr Velikii, i sooruzhennaia Im tserkov' vo imia ap. Petra (V Petrozavodskom uiezdie) [panorama]; Chugunnaia doska s nadpis'iu ob otkrytii Martsial'nykh vod; Gorod Povienets; Poselok Petrovskii Iam na Povienetsko-Sumskoi pochtovoi dorogie. Zdies' Imperator Petr Velikii izvolil probyt' sutki pri perekhodie chrez rieku Iuzhnyi Vyg s flotom i voiskom ot Bielago moria v Onezhskoe ozero v 1702 godu; Chasovnia vo imia Sv. A. Petra. V poselkie Petrovskii-Iam, na PovienetskoSumskoi pochtovoi dorogie. Osviashchena 30-go maia 1882 goda; Klimetskii Monastyr', na ostrovie Onezhskago ozera i Spasatel'naia Stantsiia; Tserkov' Uspeniia Presviatyia Bogoroditsy. V g. Vytegrie; Chasovnia na Besiednoi Gorie 
bliz g. Vytegry, osviashchennaia 30 maia 1881 goda. Imperator Petr Velikii zdes' izvolil besiedovat's krest'ianami o soedinenii rieki Volgi s riekoiu Nevoiu; Selenie Voznesen'e, pri istokie rieki Sviri iz Onezhskago ozera (V Petrozavodskom i Lodeinopol'skom uiezdakh); Selenie Vazhiny, na beregakh rieki Sviri (V Olonetskom i Lodeinopol'skom uiezdakh) [panorama]; Gorod Lodeinoe-Pole (Na lievom beregu rieki Sviri) [panorama]; Miesto byvshei Tserkvi, sooruzhennoi Imperatorom Petrom Velikim na korabel'noi verfi, v Lodeinom-Polie, v 1702 i razobrannoi v 1766 gg., okruzhennoe nasazhdennymi Im sosnami; Pamiatnik Imperatoru Petru Velikomu, v g. Lodeinom-Polie.

Petergof. St. Petersburg: P. I. Babkin, 1894.

41 mounted photographs, $33 \times 24 \mathrm{~cm}$. (Published in St. Petersburg by P. I. Babkin, with photographs by E. P. Vishniakov. Typolithography and photography printed by Shtein.) Petrodvorets (Russia).

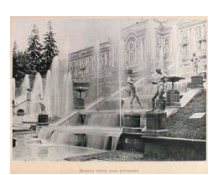

Slav. Reserve Photo (Vishniakov. Petergof) SLV 081 [Cover title]; Sobstvennyi Ego Velichestva Dvorets (po Shosse mezhdu Petergofom i Oranienbaumom); Monplezir; Eva; Doroga vdol' pruda u Marli v Nizhnem Sadu; Staryia lipy u Monplezira; Sad v Aleksandrii u Kotedzha; Pristan’ na prudu v Aleksandrii; Goticheskaia Tserkov' v Aleksandrii; Kolonada (Nizhnii sad); Shakhmatnaia gora; Vostochnyia bani (Nizhnii sad); Dvorets Tsesarevicha; Kolonada; Ploshchadka Monplezira; Zolotaia Gora; Ugol Monplezira; Dvorets skvoz' pyl' fontanov; Samson; Ol'gin ostrov; Monument Petra I-go (Nizhnii sad); Dvortsovaia Tserkov'; Bel'veder; Imperatorskii Dvorets v Aleksandrii; Pavil'on; Rimskii fontan; Na beregu moria (Nizhnii sad); Piramidal'nyi fontan; Liesnitsa iz Nizhniago Sada v Verkhnii; V Angliiskom parkie; Chast' Bel'vederskago dvortsa; Sad u Monplezira; Tsaritsyn Ostrov; Semeinyi dub v Sadu Sobstvennago Ego Velichestva Dvortsa; Marli; Bel'veder; Samson; Pavil'on; Dvorets Ego Imp. Velich. Gosudaria Imperatora Aleksandra III-go; Miesto muzyki v Nizhnem Sadu; Iskusstvennyia razvaliny; V Sobstvennom Ego Velich. parkie v Aleksandrii.

[Photographs of the Russian Imperial family held in the Slavic and Baltic Division of the New York Public Library]. St. Petersburg, 18-?

6 photographs, $24 \times 20 \mathrm{~cm}$ (with mounts). Cartes de visite of members of Russian Imperial family (one of which is produced by Bergamasco). Romanov, House of - Portraits. Russia - Kings and rulers - Biography.

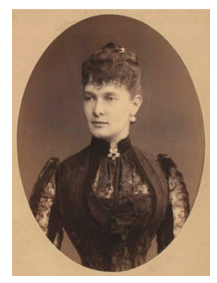

Slav. Reserve (Photo) 97-9837 SLV 086 [Aleksei Aleksandrovich, Grand Duke of Russia, 1850-1908 (brother of Alexander III)]; [Alexander III, Emperor of Russia, 1845-1894]; [Mariia Feodorovna, Empress (consort of Alexander III, Emperor of Russia), 1847-1928]; [Vladimir Aleksandrovich, Grand Duke of Russia, 1847-1909 (brother of Alexander III)]; [Mariia Pavlovna, Grand Duchess of Russia, 1854-1920 (wife of Grand Duke Vladimir Aleksandrovich)]; [Nicholas II, Emperor of Russia, 1868-1918 (son of Alexander III and Mariia Fedorovna)]. 
Pokhodnyi ochag i vagon-kukhnia. [Odessa: s.n., 1895] 4 mounted photographs: ill. diagr.; $24 \times 35 \mathrm{~cm}$. "Perl. Jun 18, 1931.” Metropolitan New York Library Council, 2008. Slavic Recon Project. Operational rations (Military supplies)-Russia. Cookery, Military- Equipment and supplies. Carriages and carts- Russia. Slav. Reserve (Photo) (Pokhodnyi ochag i vagon-kukhnya) SLV 089 Pokhodnyi ochag sistemy M. Bogaevskago. Kukhnia-dvukolka na pokhodie; Razdacha pishchi na privalie; Vagon-kukhnia. Ispytanie proizvodilos' s 30-go Sentiabria po 3-e Oktiabria 1894 g.; Vnutrennost' vagona-kukhni. Ispytanie vagona-kukhni proizvedeno pri perevozkie eshalonov 149-go piekh. Chernomorskago Ego Imperatorskago Vysochestva Velikago Kniazia Mikhaila Nikolaevicha polka, ot g. Odessy do g. Bresta.

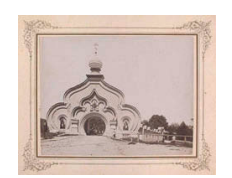

Pokrovskii Kievskii Zhenskii Obshchezhitel'nyi Monastyr'. [Kiev], [19-?]. 22 mounted photographs, $26 \times 36 \mathrm{~cm}$. Monasteries - Ukraine - Kiev. Pokrovskii Kievskii Zhenskii Obshchezhitelnyi Monastyr. Slav. Reserve (Photo) 99-5216 SLV 090 [Entrance Gate of monastery]; [Side-entrance of monastery]; [A house in the monastery and its garden]; [A path leading to the house and surrounding property]; [A building in the monastery]; [Garden in the monastery]; [Outside view of monastery]; [Wooden stairs and bridge in the garden]; [General view of main building of the monastery]; [Stone wall entrance to the monastery and two standing guards]; [The rear of the house with a fountain]; [View of house and garden]; [Wooden path leading to the monastery]; [Wooden path leading to the rear of the house]; [General view of the whole compound house and garden]; [Wide alley leading to the church]; [General view of the garden in the rear of the house]; [A path leading in the woods]; [General view of the church in the monastery]; [A different angle of the house with its elaborate architectural design]; [Wooden bridge in the garden]; [A view of the property and town's houses].

Politekhnicheskaia vystavka 1872 pochtovyi otdel. Moscow: Russkaia fotografiia, 1872. 30 mounted photographs, $32 \times 47 \mathrm{~cm}$. Cover title. Master Negative: ${ }^{\star} \mathrm{ZZ}-30916 \&{ }^{\star} \mathrm{ZQ}-$ 848. Postal service - Russia - Pictorial works. Exhibitions Moscow (Russia). Slav. Reserve (Photo) (Politekhnicheskaia vystavka 1872 goda pochtovnyi otdiel goda pochtovnyi otdiel) SLV 092 [Cover title]; Pochtovyi pavil'on na PolitekhnicheskoiVystavkie; Zdanie Moskovskago pochtamta; Iezda na oleniakh $\mathrm{v}$ Laplandii $\mathrm{v}$ Kol'skom uiezdie, Arkhangel'skoi gubernii; Rabanskii perevoz pocht chrez r. Sukhonu, Vologodskoi gubernii; Kazennyi Pochtovyi Karbas Kemskago, Kol'skago i Onegskago uiezda, Arkhangel'skoi gubernii; Pochtovaia gon'ba v Kemskom uiezdie, Arkhangel'skoi gubernii; Perevozka pochty v NizhnemNovgorodie vo vremia razliva rieki Oki; Pochtovyi Karbas Kemskago uiezda, Arkhangel'skoi gubernii; Perevozka pocht, v zimnee vremia, v Vologod. gub., 
po l'du rieki Vychegdy; Taivolaskaia pereprava pochty mezhdu Kuopio, Uleoborgom i Karelieiu, v Finliandii; Perevozka pocht po riekie Vychegdie, v Vologodskoi gubernii, v lietnee vremia; Tiflisskoe Pochtovoe Upravlenie; Terek (u seleniia Gulet na voenno-gruzinskoi dorogie); Selenie Lars (na voenno-gruzinskoi dorogie); Voenno-gruzinskaia doroga (mezhdu Baltoi i Larsom); Perenoska pochty vo vremia prekrashcheniia soobshcheniia v gorakh; Urochishche Ananur (s sievernoi storony na voenno-gruzinskoi dorogie); Dariial'skoe ushchel'e; Urochishche Ananur (s iuzhnoi storony); Gora Sion na voenno-gruzinskoi dorogie (vid s st. Kazbek); Iezda v gorakh v glubokii snieg; Ukr. Dariial'skoe na voenno-gruzinskoi dorogie; Aul Kutishi po dorogie na Gunib. Prolegaet pochtovyi trakt; Mletskii pod'em (u st. Mlet na voenno-gruzinskoi dorogie); Dariial'skoe ushchel'e (za stantsiei Lars); Selenie Sionskoe (na voenno-gruzinskoi dorogie); Perevozka pochty na Arbie; Pochtovaia stantsiia Salty po dorogie na Gunib; Miestoprebyvanie Gosudaria Imperatora Aleksandra II v 10 i 11 dni sentiabria 1871 g., vo vremia puteshestviia Ego Velichestva po Kavkazskomu i Zakavkazskomu kraiu. Dom voennago nachal'nikasredniago Dagestana na Gunibie; Dvorets Shamilia na Gunibie.

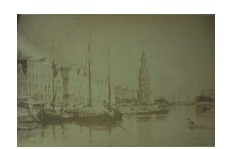

Polovtsov, Aleksandr Aleksandrocivh, 1832-1909. Pis'ma o zagranichnom puteshestvii Ego Imperatorskago Vysochestva Gosudaria Velikago Kniazia Vladimira Aleksandrocivha, 1870 goda [St. Petersburg: Pechatnia V. I. Golovina, 1871] 98 p., 6 mounted photographs, $15 \times 12 \mathrm{~cm}$. Signed: A. Polovtsov. Photographs reproduce watercolors by the painter Aleksei Bogoliubov. "Perl. Jun 18, 1931.” Metropolitan New York Library Council, 2008. Slavic Recon Project. Vladimir Aleksandrovich, Grand Duke of Russia, 1847-1909-Description and travel. Slav. Reserve (Photo) (Polovtsov) SLV 093 [A reproduction of a painting of the River Elba from the "Belle-Vue" hotel]; [A reproduction of a painting of an opera performance]; [A reproduction of a painting of a harbor]; [A reproduction of a paiting of a harbor in Amsterdam]; [A reproduction of a painting of a group of figures strolling along the beach]; [A reproduction of a landscape painting].

[Portraits of Russian Eastern Orthodox priests: collection of thirteen pictures from Russia in The Slavic and Baltic Division of The New York Public Library]. [S.1.: s.n., s.d.] [13] Photographs, 11-18 cm. Pictures of different priests taken by various photographers in different cities. A list of pictures with descriptions is included.

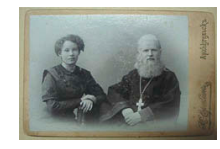

Russkaia pravoslavnaia tserkov-Clergy-Portraits. Slav. Reserve (Photo) 04-5893 SLV 094 1. [Portrait of priest and lady: workshop M. Sorokin, Arkhangel'sk]; 2. [Portrait of priest: workshop M. Sorokin, Arkhangel'sk (verso under text: $\mathrm{Na}$ dobruiu pamiat' Ivanu Vasil'evichu Klestovu. Sviashch. Koz'ma Ovchinnikov. 2 iiunia, 1909 g.)]; 3. [Portrait of priest: workshop Ia. 
Leitsinger, Arkhangel'sk (verso under text: Dariu v znak dobroi pamiati glubokochtimomu Ivanu Vasil'evichu Klestovu. 15 iiunia, 1909 goda. Vsegdashnii Vash Molitvennik Solov. Mon-yria. Ieromonakh Paranii)]; 4. [Portrait of priest: workshop Ia. Leitsinger, Arkhangel'sk (verso under text: $\mathrm{Na}$ dobruiu pamiat' Glubokouvazhaemomu Ivanu Vasil'evichu Klestovu. Solov. Mon. Riznichii Ieromonakh David, 31 mai, 1905 god)]; 5. [Portrait of priest: workshop G. Soberg, Arkhangel'sk (verso under text: Na dobruiu pamiat' N. V. Klestovu ot monakha Alimpiia. 12 iiunia 1909 goda, Solovki)]; 6. [Portrait of priest: workshop P. Afonasev, Biisk (verso under text: Sviashchennik Vladimir Ivanovich Vasil'ev. 1910 goda avgusta 8-go dnia)]; 7. [Portrait of priest: workshop L. Raevskii, city Vologda (verso under text: Druziam moim - dobreishim Ivanu Vasil'evichu i Anastasii Iakovlevne Klestovym na dobruiu pamiat' 22 dekabria 1918 goda (4 ianvaria) 1919 goda)]; 8. [Portrait of priests: workshop Bogoiavlenskoi, Ves'egonsk (on bottom: Sviashch. sela Vauch Joann Stratonovskii rod. 20 Fevr. 1845, + 2 marta 1900 g. Sviashch. sela Nikoly-Ramen'ia (?) Vladimir Stratanovskii)]; 9. [Portrait of priest: workshop N. Dorofeev, city S. Peterburg (verso under text: 1909 god. 7 iiunia. Na dobruiu pamiat' Ioannu Vasilevichu G-nu Klestovu ot Ierodiakona Zosimy)]; 10. [Portrait of priest: workshop N. Dorofeev, S. Peterburg]; 11. [Portrait of priest: workshop V. Egorov, S. Peterburg (verso under text: Georgii Basovich)]; 12. [Portrait of priest and three men in secular dress: workshop Cabinet-portrait (verso under text: $\mathrm{Na}$ dobruiu molitvennuiu pamiat' uvazhaemomu o Khristov Bratu Ivanu Vasil'evichu Klestovu ot V.A. Kolovalova, m.p. Busheva. G. O. Kliashenok. 25 oktiabria 1907 g., S. Peterburg]; 13. [Portrait of priest, O. G. Petrov: Post-card].

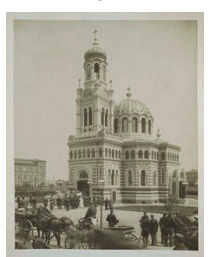

Pravoslavnaia tserkov' $v$ gorodie Lodzi. Lodz, 1884. 2 p. printed text, 5 mounted photographs, $27 \times 37 \mathrm{~cm}$. "Perl. Jun 18 1931." 5 uncaptioned photographs showing exterior and interior views of the Russian Orthodox church in Lodz. Accompanying text deals with the history of this church. Bound in lime-green crushed velvet with silk moire lining. Church architecture - Poland - Lodz. Slav. Reserve Photo (Pravoslavnaya tserkov v gorodie Lodzi) SLV 096 [Cover title]; [Church of Grand Duke Alexander Nevsky in Lodz]; [Church of Grand Duke Alexander Nevsky in Lodz]; [Church of Grand Duke Alexander Nevsky in Lodz]; Interior view of the Church of Grand Duke Alexander Nevsky]; [Interior view of the Church of Grand Duke Alexander Nevsky].

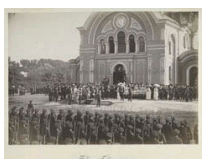

Prebyvanie ikh Imperatorskikh Velichestv $v$ Brest-Litovske, $\boldsymbol{v}$ Vysoko-Litovske i Spale. Warsaw: A. Kantor, [188-?]. 38 mounted Photographs, $56 \times 42 \mathrm{~cm}$. "Perl. Jun 18, 1931." "Kollektsiia fotograficheskikh snimkov sdielannykh Mechkovskim." Binder's title. 53 negative fim plates are boxed. Some photographs are marked "Dèposê Mechkowski." Metropolitan New York Library 
Council, 2008. Slavic Recon Project. Alexander III, Emperor of Russia, 18451894 - Travel - Poland. Alexander III, Emperor of Russia, 1845-1894 Travel - Poland - Spała (Piotrków Trybunalski) Brest (Belarus). Spała (Piotrków Trybunalski, Poland). Slav. Reserve (Photo) (Prebyvanie ikh Imperatorskikh Velichestv v Brest-Litovske, v Vysoko-Litovske i Spale) SLV 097 Brestskii vokzal; Triumfal'nyia vorota v Breste-Litovskie; Prazdnik Borodinskago polka; Prazdnik Borodinskago polka; Prazdnik Borodinskago polka; Prazdnik Borodinskago polka; Prazdnik Borodinskago polka; Artilleriiskii lager' v ozhidanii Ikh Velichestv; Pochetnyi karaul. Osmotr artileriiskikh orudii; Parad v Vysoko-Litovskie; Parad v Vysoko-Litovskie; Parad v Vysoko-Litovskie; Parad v Vysoko-Litovskie; Parad v VysokoLitovskie; Aerostat na manevrakh; Proshchal'nyi zavtrak na myzie Shestakovo; Mechkovskii v ambulanse $\mathrm{v}$ kachestve korrespondentafotografa "Vsemirnoi Illiustratsii"; Voennyi lager' v Vysoko-Litovskie; Epizod iz manevrov. Dvorets v Vysoko-Litovskie; Obshchii vid Spaly; Kabinet Gosudaryni v Spalie; Utro v Spalie; Ot'iezd Gosudaria na okhotu; Ot"iezd Gosudaryni na okhotu; Ot'iezd Gosudaryni na okhotu; Otezd Gosudaryni na okhotu; Posle okhoty v Spalie; Vecher v Spalie vo vremia zakata solntsa; Ugoshchenie dietei; Ugoshchenie dietei; Dietskie khory; Dietskie khory; Dietskie khory; Detskie khory; Podnesenie Gosudaryne buketa i polotentsev. Gosudar'sredi dietei; Gosudar'sredi dietei; Voennye orkestry v Spalie. Khor Tomashovskoi pozharnoi druzhiny; Dvorets v Spalie v to vremia kogda Ikh Velichestva nakhodiatsia na okhotie.

Prebyvanie Imperatora Vil'gel'ma II $v$ Rossii. 1888 (r) 19 g. [St. Petersburg: Fotograf N. Digo, 1888?] 49 mounted photographs, $35 \times 24 \mathrm{~cm}$. Title from original portfolio. "Fotograf. N. Digo." "Perlstein Jun 18, 1931.” Metropolitan New York Library Council, 2008. Slavic Recon Project. William II, German Emperor, 1859-1941 Travel - Russia. Alexander III, Emperor of Russia, 1845-1894. Visits of state -Russia. Slav. Reserve (Photo) (Prebyvanie Imperatora Vilgelma II v Rossii) SLV 098 [Cover title]; Russkaia eskadra na Bol'shom Kronshtadtskom reidie.; Imperatorskaia iakhta Aleksandriia, iakhta Striel'na; Minonostsy; Voennaia pristan' v Petergofie; Kreiser, Aziia, Imp. Germ. iakhta Gogentsollern, Derzhava; Gogentsollern, Aziia, Derzhava; Gertsog Edinburgskii, 3 klipera, Minin.; 3 klipera. Minin.; Bunchuk, Strel'na, Aleksandriia, Aziia, Anterpreis.; Imperatorskaia iakhta Aleksandriia; Imperatorskaia iakhta Tsarevna, Dnepr, 2 monitora, 2 lodki; [No caption]; Chastnyia suda, provozhaiushchiia iakhtu Aleksadriiu, s Ikh Velichestvami, ot Kronshtadta do Petergofa; Minonosotsy, Minonoski, Rybka; Imperatorskaia iakhta Derzhava; Leitenant Il'in, Mandzhur, Boiarin, Baian, Skobelev, Adm. Lazarev, Adm. Chichagov, Gerts. Edinburgskii; Oprichnik, Plastun, Viestnik, Minin.; Tsarevna; Russkaia eskadra na Bol'shom Kronshtadtskom reide; Voennaia pristan' v Petergofie, vodopad 
Sampson; Ikh Velichestva Imperatory Aleksandr III i Vil'gel'm II i Prints Genrikh Germanskii so svitoiu, pri prokhozhdenii voisk, na parade v Krasnom Selie; Tsarskii valik; Ikh Velichestva skhodiat s Tsarskago valika, posle zavtraka; Priiezd Eia Velichestva k Tsarskomu valiku; Ikh Velichestva i Prints Genrikh vkhodiat na Tsarskii valik; Tsarskii valik; Zavtrak gg. ofitserov u Tsarskago valika; Vyzov nachal'nikov chastei dlia provozglasheniia tosta za Imperatora Vil'gel'ma II; Vyzov nachal'nikov chastei dlia provozglasheniia tosta za Imperatora Vil'gel'ma II; Tsarskii valik; Ikh Vysochestva skhodiat s Tsarskago valika, posle zavtraka; Germanskaia eskadra; Imperatorskaia Germanskaia iakhta Gogentsollern; Germanskaia eskadra vo vremia saliuta; Petergofskaia voennaia pristan'; Germanskii brononosets Kaizer; Peterburg; Gogentsollern, Aziia; Prints Adal'bert, Gneizenau; Germanskaia eskadra; Kliper; Voennaia pristan' v Petergofie, ot'iezd Germ. Imperatora; [Yacht]; Neva; Aleksandriia; Gogentsollern vo vremia proshchal'nago zavtraka; Ikh Velichestva skhodiat s Tsarskago valika, posle zavtraka; Germanskaia eskadra. Saliut; Baden; Anterpreis, amerikanskoe sudno.

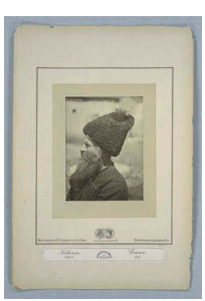

Raoult, J. S. Nieskol'ko narodnykh tipov Rossii, udostoennyi Vysochaisheiu blagodarnost'iu i podarkom Ego Imperatorskago Velichestva $=$ Quelsques types des peuples de la Russie. [Odessa: Lith. N. Beckel, 18-]. 2 v., 159 mounted photographs, $34 \times 50 \mathrm{~cm}$. Album of photographs with descriptive letterpress in French and Russian, bound in 2 vols. No text. "Mention honorable de S.M. l'Empereur de Russie. Photographé J.S. Raoult, Odessa." With bookplate of Emperor Alexander III. DOE Title II-C "Russian Illustrated Books and Photographs" Project. Costumes - Russia. Peoples - Russia. Slav. Reserve (Photo) 93-3746 SLV 099 Nieskol'ko narodnikh tipov Rossii. Chast' I. [Cover title]; Bolgarin; Bolgary; Bolgarki; Bolgarki; Bolgarka; Bolgarka; Bolgary; Bolgarka; Bolgary; Bolgarin; Tul'skoi gubernii; Tul'skoi gubernii; Tul'skoi gubernii; Tul'skoi gubernii; Kazansk. Gubernii. Tatary; Kazansk. Gubernii. Tatarin; Kazansk. Gubernii. Tatarin; Kazansk. Gubernii. Tatarka; Kazansk. Gubernii. Tatarin; Kazansk. Gubernii. Tatarka; Kazansk. Gubernii. Tatary; Kazansk. Gubernii. Tatarka; Kazansk. Gubernii. Tatary; Kazansk. Gubernii. Tatarki; Orlovskoi gubernii; Orlovskoi gubernii; Orlovskoi gubernii; Orlovskoi gubernii; Orlovskoi gubernii; Orlovskoi gubernii; Orlovskoi gubernii; Orlovskoi gubernii; Orlovskoi gubernii; Orlovskoi gubernii; Orlovskoi gubernii; Poltavskoe gubernii; Poltavskoe gubernii; Poltavskoe gubernii; Poltavskoe gubernii; Poltavskoe gubernii; Poltavskoe gubernii; Poltavskoe gubernii; Poltavskoe gubernii; Rumyny; Rumyny; Rumyny; Rumyny; Rumyn; Rumyn; Rumyny; Rumynka; Rumyn; Rumynki; Rumynsk. sviashchennik; Rumynka; Bukharets; Persiiani (Russkopoddannye); Konvoi Ego Imperat. Velichestva; Konvoi Ego Imperat. Velichestva; Krymskie tatary; Krymskie tatary. Shkola; Krymskaia tatarka; Krymskoi tatarin; Evrei; Evrei; Arkhimandrit; Donskoi kozak; Bessarabskoi 
gub. Evrei; Bessarabskoi gubernii; Bessarabskoi gubernii; Bessarabskoi gubernii; Bessarabskoi gubernii; Bessarabkoi gub. Evrei; Bessarabskoi gubernii; Bessarabskoi gubernii; Bessarabskoi gubernii; Bessarabskoi gubernii; Bessarabskoi gubernii; Simbirskoi gubernii. Cheremisy; Simbirskoi gubernii. Cheremisy; Simbirskoi gubernii. Cheremisy; Simbirskoi gubernii. Cheremisy; Simbirskoi gubernii. Cheremisy; Simbirskoi gubernii. Cheremisy.

Chast' II [Cover title]; Nizhegorodskoi gubernii; Nizhegorodskoi gubernii; Nizhegorodskoi gubernii; Nizhegorodskoi gubernii; Khersonskoi gubernii; Khersonskoi gubernii. Evrei; Khersonskoi gubernii; Khersonskoi gubernii; Khersonskoi gubernii. Evrei; Khersonskoi gubernii. Evrei; Khersonskoi gubernii; Chernigovskoi gubernii; Chernigovskoi gubernii; Chernigovskoi gubernii; Chernigovskoi gubernii; Kurskoi gubernii; Kurskoi gubernii; Kurskoi gubernii; Ekaterinoslavskoi gub; Ekaterinoslavskoi gub; Tambovskoi gubernii; Podol'skoi gubernii; Podol'skoi gubernii; Podol'skoi gubernii; Podol'skoi gubernii; Podol'skoi gubernii; Podol'skoi gubernii; Podol'skoi gubernii; Podol'skoi gubernii; Podol'skoi gubernii; Kievskoi guvbernii; Kievskoi guvbernii; Kievskoi guvbernii; Kievskoi guvbernii; Kavkaz (Mingrelets); Kazkaz. Kubanskie kozaki (Kavaleriia); Kazkaz. Kubanskie kozaki (Plastuny); Kavkaz. Imeretiny; Kavkaz. Imeretiny; Kavkaz. Gruziny; Kavkaz. Mingrel'tsy; Kavkaz. Imeretiny (Druzhina Kavaleriia); Kavkaz. Evrei; Kavkaz. Gruziny; Kavkaz; Kavkaz. Guriitsy; Kavkaz. Sviashchennik; Kavkaz. Gruzin; Kavkaz (Mingrelets); Kavkaz. Imeretin; Kavkaz. Evrei; Kavkaz. Gruzin; Kavkaz. Muzykant; Kavkaz. Guriits; Kavkaz (Mingrelets); Kavkaz. Gruzinka; Kavkaz. Imeretinka; Kavkaz. Guriitsy (Druzhina); Kavkaz. Guriitsy (Druzhina); Kavkaz. Mingrel'tsy; Kavkaz. Imeretin; Kavkaz. Svanety; Kavkaz. Guriits; Kavkaz. Imeretiny; Kavkaz. Imeretin; Kavkaz. Evrei; Kavkaz. Guriitsy; Kavkaz. Evrei; Kavkaz. Gruzin; Kavkaz. Gruzinka; Kavkaz. Kubanskii kozak; Kavkaz. Armianin; Kavkaz. Evrei; Kavkaz (Mingrelets); Kavkaz. Guriitsy; Kavkaz. Imeretiny; Kavkaz. Khefsur; Kavkaz. Khefsur.

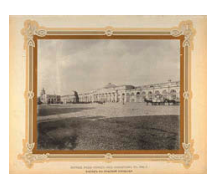

Razmadze, A. S. Torgovye riady na Krasnoi ploshchadi $v$ Moskvie. Kiev: Izd. pravlenii obshchestv verkhnikh i srednikh riadov, 1893. 64 p.: ill., plates; $31 \times 39 \mathrm{~cm}$. "(Dieloproizvoditelia Obshchestva verkhnikh i srednikh riadov...)"-t.p. Text within ornamental borders. Plates printed on both sides. Foto-litho tip. zavedenii S. V. Kul'zhenko. Presented to Grand Duke Vladimir Aleksandrovich. DOE Title II-C "Russian Illustrated Books and Photographs" Project. Reproductions of photographs by E. Simonov, and of other illustrations. Describes work on shops on Red Square in Moscow, undertaken in 1888. Torgovye riady (Moscow, Russia) Trade associations - Russia (Federation) Moscow. Slav Reserve (Photo) (Razmadze. Torgovye riady) SLV 121 [Cover title]; [Title page]; Kreml' i Krasnaia Ploshchad'; Krasnaia ploshchad' v kontsie XVIII vieka; Verkhnie riady pered ikh zakrytiem v 1888 g. Fasad na Krasnoi Ploshchadi; Verkhnie riady pered ikh zakrytiem v 1886 g. Fasad 
po Il'inkie; Verkhnie riady pered ikh zakrytiem v 1886 g. Vetoshnyi riad; Verkhnie riady pered ikh zakrytiem v 1886 g. Vid krysh; Starye verkhnie riady $\mathrm{v}$ torgovoe vremia. Bol'shoi sukonnyi riad; Verkhnie riady pered slomkoi. Nozhevaia liniia; Verkhnie riady pered slomkoi. Uzen'kii riad; Verkhnie riady pered slomkoi. Srednii prokhod; Verkhnie riady pered slomkoi. Sredina kholshchevago riada; Slomka starykh verkhnikh riadov. Obshchi vid raskopki drevnikh podvalov; Slomka starykh verkhnikh riadov. Dvukhiarusnyi drevnii podval; Zakladka novykh zdanii verkhnikh riadov 21-go Maia 1890 g.; Postroika novykh zdanii verkhnikh riadov. 3-i passazh; Postroika novykh zdanii verkhnikh riadov. Okonchanie 3-go etazha 3-i linii; Postroika novykh zdanii verkhnikh riadov. 4-ia liniia i malyi korpus; Postroika novykh zdanii verkhnikh riadov. Proizvodstvo rabot vnutri sredniago passazha; Fasad malago korpusa; Vnov' ustroennyi vetoshnyi proiezd; Vid krysh i svietovykh perekrytii novykh zdanii verkhnikh riadov; 3-ii passazh v novykh verkhnikh riadakh; Glavnyi vestibiul' vetoshnago proiezda; 3-ii podval'nyi passazh; Verkhnie riady po Il'inkie; Vnutrennost' magazina gg. Br. Sapozhnikovykh; Verkhnie riady. Odin iz bokovykh poperechnykh passazhei; Novye srednie riady. Razriez zdanii; Postroika novykh srednikh riadov. Zakladka zdanii 18-go Iiulia 1891 g.; Postroika novykh srednikh riadov. Naruzhnyi korpus na uglu Il'inki; Postroika novykh srednikh riadov. Naruzhnyi korpus po dlinie Moskvorietskago proiezda; Postroika novykh srednikh riadov. Vozvedenie srednikh nadvornykh korpusov; Glavnyi fasad zdanii srednikh riadov po Moskvorietskomu proiezdu; Verkhnie riady. Glavnyi fasad.

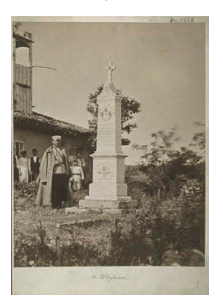

Risunki pamiatnikov chinam 12-go korpusa, pavshim v voine 1877-78 gg. $v$ Bolgarii. [S.l.: s.n.,, 1878?]. 24 mounted photographs, 26 x $33 \mathrm{~cm}$. "Perl. Jun 18, 1931." Title from original purple plush portfolio. Captions in manuscript. Metropolitan New York Library Council, 2008. Slavic Recon Project. RussoTurkish War, 1877-1878 - Monuments - Bulgaria. RussoTurkish War, 1877-1878 -Bulgaria. Slav. Reserve Photo (Risunki pamiatnikov...) SLV 100 [Cover Title]; s. Pirgos; s. Trestenik; s. Trestenik; s. Mechka; s. Mechka; s. Pirgos; s. Trestenik; s. Mechka; s. Mechka; s. Mechka; s. Trestenik; s. Mechka; s. Katselevo; s. Obertenik; m. Biela; s. Ablavo; Iovan-Chuflik; s. Trestenik; s. Ablava; s. Trestnik; Ferendzhik; s. Ablava; s. Nisovo; s. Mechka.

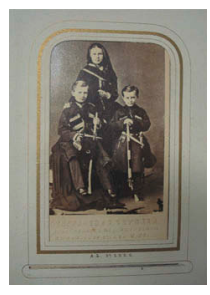

[Romanov Family photo album: 1860s-1870s]. [Russia: s. n., 186-?-187-?] 39 photographs: all ports.; $12 \times 17 \mathrm{~cm}$. Octavo, brown embossed morocco, metal plates. Includes portraits of Grand Duchess Maria Aleksandrovna Romanov (1824-1880), consort of Emperor Alexander II, and her sons Grand Dukes Sergei Aleksandrovich (1857-1905) and Pavel Aleksandrovich (1860-1919). Konstantin Collection. Romanov, House of- 
Portraits. Pavel Aleksandrovich, Grand Duke of Russia, 1860-1919-Portraits. Sergei Aleksandrovich, Grand Duke of Russia, 1857-1905-Portraits. Mariia Aleksandrovna, Empress, consort of Alexander II, Emperor of Russia,-1824 1880-Portraits. Photograph albums-Russia-Specimens. Slav. Reserve (Photo) 05-2970 SLV 101

1. Aleksandr II; 2. Mariia Aleksandrovna; 3. Gosudar' naslednik Tsesarevich; 4. Velikaia kniaginia Maria Aleksandrovna; Velikie kniaz'ia Sergei i Pavel Aleks.; 5. Velikie kniaz'ia Sergei i Pavel Aleks.; 6. Vel. kn. Sergei Aleksandrovich; 7. Vel. kn. Nikolai Konstantinovich; 8. Velikaia kniaginia Aleksandrovna Iosifovna i vel. kniaginia Olga Konstantinovna; 9. Vel. kn. Olga Konstantinovna; 10. Anmama [?], d. Nikola, t. Ol'ga, t. Vera i papa?; 11. Vel. kniaz'ia Dmitrii, Konstantin i Viacheslav Konstantinovichi; 12. Vel. kn. Mikhail Nikolaevich; 13. Vel. kniaginia Ol'ga Fedorovna; 14. Vel. kn. Nikolai Mikhailovich; 15. Aleksandra; 16. Nikolas; 17. La grande Duchesse Alexandra Iosifovna; 18. La grande Duchesse Alexandra Iosifovna; 19. Grand Duke Nikoai Constantinovich; 20. [No information written]; 21. G.D. Constantine et Dmitri Constantinovich; 22. Dimitry Konstantinovich; 23. G.D. Alexandra Iosifovna et G. Dushesse Olga Constantinovna; 24. Alexandra Iosifovna i Olga Konstantinovna; 25. Alexandra Iosifovna i Olga Konstantinovna; 26. [No information. Probably Aleksandra. Iosifovna]; 27. [No photo]; 28. Rai de grece; 29. G.D. Elisabeth...; 30. A.J.; 31. Korol' Grecheskii Georg; 32. La Grande Duchesse Olga Constantinovna de Russie; 33. Vel. kniaginia Anastasia Mikhailovna; 34. Vel. kniaginia Mariia Nikolaevna; 35. Vel. kniaginia Ol'ga Nikolaevna, Reine de Wurtemberg; 36. la G.D. Marie de Russie, fille [Velikaia kniaginia Mariia Nikolaevna]; 37. Vel. kniaginia Aleksandra Petrovna; 38. Doch’ Vel. kn. Marii Nikolaevny Eugeniia Maksmilianovna; 39. G.D Duchess [?] Constantinova; 40. [No information].

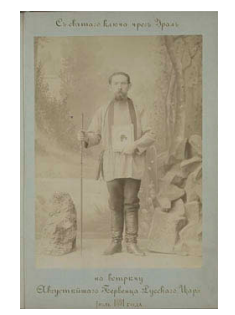

Rostovtsev, Gorodskoi Golova. Pervyi sviatoi i serdechnyi dolg viernopoddannykh Russkago Tsaria ili vstriecha deputatsiei ot goroda Sterlitamaka Ego Imperatorskago Vysochestva Gosudaria Nasliednika Tsarevicha Nikolaia Aleksandrovicha... G. Sterlitamak Ufimskoi gubernii: Tip. A. Busgina, 1891. 58 printed, 10 typescript p. [3] mounted photographs; $19 \times 28 \mathrm{~cm}$. Presented to Grand Duke Vladimir Aleksandrovich by the author. Silk moiré endsheets with embossed dedication to Grand Duke Vladimir Aleksandrovich. With related text dealing with the assassination attempt on Tsesarevich Nikolai in Japan in 1890. With second t-p: Ego Imperatorskomu Vysochestvu Gosudariu Nasliedniku Tsesarevichu i Velikomu Kniaziu Nikolaiu Aleksandrovichu Viernopoddanieishii Adres Povergaetsia Steplitamakskim Gorodskim Goloviu Rostovtsevym. Perlstein stamp: "Perl. Jun 18, 1931.” Metropolitan New York Library Council, 2008. Slavic Recon Project. Icons, Russian. Nicholas II, Emperor of Russia, 1868-1918. Slav. Reserve (Photo) (Rostovtsev. Pervyi sviatoi serdechnyi dolg) SLV 102 
[Cover title]; S sviatago kliucha chrez Ural na vstriechu Avgusteishago Perventsa Russkago Tsaria. Iiul' 1891 goda; Pred Avgusteishim Perventsom Russkago Tsaria 23 Iiulia 1891 goda; K sviatomu kliuchu na osviashchenie obraza Chudotvornoi Ikony Podnosimago Batiushkie Tsariu i Matushkie Tsaritsie v pamiat' dnia 28 okt. 1866-1891.

Rudnev, D. Al'bom poiezdki komandy Sieverskago dragunskago polka iz goroda Vladikavkaza, cherez glavnyi khrebet Kavkazskikh gor, $v$ g. Gori i obratno. Vladikavkaz: Tipolitografiia Z. Shuvalova, 1886. 57 mounted photographs, $44 \times 34 \mathrm{~cm}$. Marked “Dec. 8, 1933." Metropolitan New York Library Council, 2008. Slavic Recon Project. Russia. Armiia. Sieverskii dragunskii polk. Voenno-Gruzinskaia doroga (Georgia and Russia). CaucasusDescription and travel. Slav. Reserve (Photo) (Rudnev. Albom poezdki) SLV 103 [Lida?] uchastvovavshikh $\mathrm{v}$ poiezdke; Vladikavkaz gora stolovaia i kazbek; Kavkazskoi artilleriiskoi polygon;

Kriepostnaia batareia kavkazkago voennago okruga; Slobod. Alagir; [Fortress?]; Voenno Osetinskoe shosse; Ardonskoe ushel'e; Nizhnii unal; Urochishche sv. Nikolaia; Sadonskiia rudniki; Nuzal'skii most; Aul Nueal; Narskoe ushchel'e; Narskoe ushchel'e aul Tsmi; Narskoe ushel'e aul Nar.; Narskoe ushchel'e aul Slias; S vysoty rokskago perevala r. Ruch'-Don derevni Roki; Ushchel'e Liakhvy bliz Koshki; Pod"em na rokskii pereval; Aul Slias; Bivyak u aula Noa-kau; G. Ardon Sv. Nikolaia; Sievernyi sklon roks. perevala v iiul. 15; Ushchel'e r. Liakhvy; Prival pod viekovymi oreshnikami derev. Kekhvi po r. Liakhve; ...monastyr'; Selenie Tskhinval; Derevnia Karalety dom kniazia Revaza Eristova; Gor. Gori i drevnei krieposti; Monastyr' sv. Georgiia prival na Lomiiskom perevale; Voenno gruzinskaia doroga Mlety; Voen. gruz. dor. st. Gudaur; Voenno gruzinskaia doroga chertova dolina; Selenie Kobi; Stantsiia Kobi i gora Sion; Kobi drevniaia tserkov' s. Georgiia; Trusovskoe ushchel'e aul Abano; Gora Zil'gikhokh istoki r. Tereka; Tyrsko-zakskii pereval; Zakskoe ushchel'e aul Abaity Kau; S vysoty gory Styr-Khokh zakskoe ushchel'e; Ledniki na gore Khiladzhi Khokh; Vodopad Baratinskago; Kurtatinskoe ushchel'e aul Khidikus; Nuzal'skaia drevniaia tserkov'; Voen. gruz. dor. most iz Gudaura v Kobi; Sievernyi sklon Styr Khokhskago perevala; Kurtatinskoe ushchel'e po r. Fiak-Don; S vysoty Styr-Khokho ledniki na g. KhiladzhiKhokh; Akhal-Gori d. kniazia Eristova; Most iz Kobi v Gudaur; Kobi vstrecha voennago Ministra; Alligi[?] -srebro-svintsovyi zavod; Dnevka v g. Gori u Kniazia Ivana Givicha Amilakhvari; Ushchel'e r. Liakhvtsy derev. Khvtse; Tipy kavkaza Russia. Komissiia dlia issliedovaniia Khar'kovoNikolaevskoi zheleznoi dorogi. 
Prilozhenie $k$ otchetu Vys. uchrezhdennoi kommissii dlia issliedovaniia Kharkhovo-Nikolaevskoi zhelieznoi dorogi: fotograficheskie snimki zheliezno-dorozhnykh sooruzhenii. [Russia, 188-?]. 35 mounted photographs, $44 \times 32 \mathrm{~cm}$. Album of photographs with captions in manuscript. Title from the shelflist card. Text on verso of plate 1: "Dnieprovskii most v gor. Kremenchugie. Samoe bol'shoe iskusstvennoe sooruzhenie dorogi. Most mnogoraskosnoi system v 21 prolet po 41 sazh. Sluzhit dlia dvizheniia poiezdov, ekipazhnago i pieshekhodnago soobshcheniia."

Markings: "Oct. 19, 1936." Photographs nos. 11-12 and 32 wanting. Metropolitan New York Library Council, 2008. Slavic Recon Project. Kharkovo-Nikolaevskaia zhelieznaia doroga. Railroads -Ukraine Mykolaïvskyi raion (Lvivska oblast). Railroad stations - Ukraine. Railroads - Ukraine - Kharkiv. Slav. Reserve (Photo) (Russia. Komissiia dlia issledovaniia Kharkovo-Nikolayevskoi zheleznoi dorogi) SLV 105 Dnieprovskii most (fasad) [text on verso]; Dnieprovskii most (vid s boku); Sul'skii most na 196 ver. Romenskago uchastka (polovina so storony Romen) [text on verso]; Sul'skii most (polovina so storony Kremenchuga); Kontsevaia chast' Sul'skago mosta; Most s shandorami chrez r. Krivuiu Rudu pod chetyre puti st. Kremenchug [text on verso]; Most otv. 4 na 74 ver. Romenskago uchastka [text on verso]; Most vodospusk na 109 ver. Romenskago uchastka.[text on verso]; Puteprovod na 197 ver. Romenskago uchastka [text on verso]; Puteprovod na 199 ver. Romenskago uchastka [text on verso]; Kazarma [text on verso]; Zhiloi dom na st. Veselyi-Podol [text on verso]; Zhiloi dom na st. Liubotin [text on verso]; Zhiloi dom sluzhby puti na st. Romny [text on verso]; Zhiloi dom sluzhby puti i pochtovoe otdelenie na st. Kremenchug [text on verso]; Shkola na st. Liubotin [text on verso]; Passazhirskoe zdanie st. Kremenchug I klassa [text on verso]; Passazhirskoe zdanie st. Liubotin [text on verso]; Passazhirskoe zdanie st. Kobeliaki [text on verso]; Passazhirskoe zdanie st. Koviagi [text on verso]; Passazhirskoe zdanie st. Ryzhov (so storony Liubotina) [text on verso]; Passazhirskoe zdanie st. Ryzhov (so storony Khar'kova); Passazhirskoe zdanie st. Romodan. [text on verso]; Passazhirskoe zdanie st. Romodan (so storony dvora); Passazhirskoe zdanie st. Khorol [text on verso]; Passazhirskoe zdanie st. Globino IV kl. [text on verso]; Vodonapornaia bashnia na st. Poltava [text on verso]; Vodoemnoe zdanie na st. Veselyi-Podol [text on verso]; Vodoemnoe zdanie na st. Romodan [text on verso]; Parovoznoe depo na st. Liubotin [text on verso]; Masterskaia na st. Liubotin [text on verso]; Masterskaia i reservuary dlia legko-vosplameniaiushchikhsia veshchestv na st. Liudomin [text on verso]; Masterskaia na st. Poltava [text on verso]; Passazhirskii parovoz [text on verso]; Tovarnyi 8-mi kolesnyi parovoz [text on verso]. 


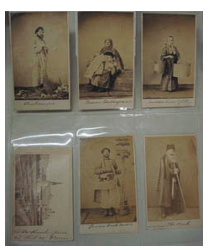

[Russian types: five photographs]. Moscow: Novaia fotografiia Konarskago, 1870? 6 photographs, $11 \times 6 \mathrm{~cm}$. Carte-de-visite mounted on cardboard, five with English, one with French handwritten captions, and one with Konarskii's printed device on verso. Five photographs of street types and one View of the Kremlin. Purchased from Bernard J Shapero Rare Books, 2007. Costume-Russia-19th century. Kremlin (Moscow, Russia). PhotographyRussia-19th century. Konarskii, M. Slav. Reserve (Photo) 07-6538 SLV 108

1. Street Sweeper; 2. The Washerwoman; 3. Russian servant girl; 4. View of the Kremlin from Bolshoi Kamenniy bridge; 5 . Russian brush vendor; 6 . The Monk.

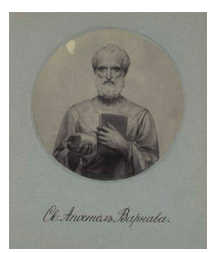

S risunkov profes. M. N. Vasil'eva dlia Khrama Spasitelia $\boldsymbol{v}$ Moskvie. [Russia?: s.n., 18-]. 34 photographs, $19 \times 22 \mathrm{~cm}$. Purchased from Christie's. Paul M. Fekula Collection. Cover title with golden letters on leather. Photographs mounted on leaves with golden finish. Manuscript descriptions of figures on verso. Originally a presentation copy to "NVD." Vasilev, M. N. Khram Khrista Spasitelia (Moscow, Russia). Murals-Russia-Moscow. Slav. Reserve (Photo) 94-2564 SLV 109 [Cover title]; [Archangel]; Sv. Nikolai Sviatosha; Sv. Roman Riazanskii; Sv. Efrosiniia Polotskaia; Sv. Petr Muromskii; Sv. Gavriil Pskovskii; Sv. Iulianiia Ol'shanskaia; Sv. Ioann Uglitskii; Sv. Fevroniia Kniaginia Muromskaia; Sv. Apostol Varnava; Sv. Sviashchennomuchenik Dionisii Areopagit; Sv. muchenik Iustin Filosov; Sv. Leonid muchenik iz Ellady; Sv. Apostol Sila; Sv. Ravnoapostol'nia Fekla; Sv. Velikomuchenik Dimitrii Solunskii; Sv. Avenir. Tsar Indiiskii; Sv. Ierofei Afinskii. Sv. Afenogen Episkop; Sv. Prokl Tsaregradskii. Sv. Efrem Sirin; Sv. Sofronii Ierusalimsk; Sv. Amvrosii Mediolan; Sv. Feodor Studit. Sv. Iosif Studit; Sv. Grigorii Neokesariisk; Sv. Mefodii Potarsk; Sv. Roman Diakon. Sv. Anatolii Tsaregradsk; Sv. Kos'ma Maiumskii. Sv. Ioann Damaskin; Sv. Feofan Sladkopievets; Sv. Iosif Piesnopisets; Sv. Arsenii Konevskii; Sv. Stefan Makhriskii; Sv. Nikon Radonezhskii; Sv. Antonii Siskii; Sv. Andronnik Moskovskii; Sv. Mitrofan Voronezhskii; Sv. Inokentii Irkutskii; Sv. Nil Stolobenskii; [Archangel].

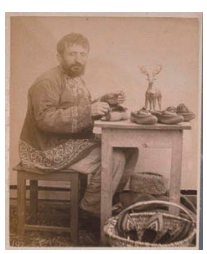

Sakhalin, the island of exile: Photograph collection of the Russian island penal colony during the late 19th and early 20th centuries. [S.l., s.n.], 1894-1905. 129 mounted photographs: ill. (map); $31 \times 40 \mathrm{~cm}$. The collection is mentioned in the magazine Bostonia, no. 3 (May/June, 1990), p. 53-59. Views of administrative center of Alexandrovsk and Derbinsk on Sakhalin Island. Microfilm: ${ }^{\star} Z Q-1002$. "Typical views include administrative buildings, churches, local ethnic types, prisoners (some mention crimes, punishments) convicts at work. Photos numbered in no particular order, with English captions, with some corrections by a person (the photographer?) who was present during the photography, and thus knew the 
subject matter/sites. Four per page of $41 / 4$ " x 5 1/2" prints (128 total, some on verso). No photographer given. Photos presumably taken around time of Chekhov's visit and composition of his famous expose Journey to Sakhalin (1895). Remarkable materials from one of Russia's most notorious penal colonies. Of exceptional interest, in spite of the fading of some of the prints. The photographs themselves are generally well-executed. Priority for preservation; high quality copy negatives essential." (William C. Brumfield) Some handwriting in captions resembles that of George Kennan. A "photographer Budogionc" is mentioned in a caption.

Sakhalin (Sakhalinskaia oblast, Russia). Prisoners - Russia (Federation) Sakhalin (Sakhalinskaia oblast). Political prisoners -Russia (Federation) Sakhalin (Sakhalinskaia oblast). Slav. Reserve (Photo) (Sakhalin) SLV 110 Church at Post Alexandrofsk (10); Scene in Port Alexandrofsk on the Island of Sahalin [Sakhalin] (104); Chapel at Post Alexandro[v]sk (66); The house of the Governor of Sahalin [Sakhalin] at Alexandrofsk (204); Village Street (149); Street in Post Korsako[v]sk (139); Church at Post Korsakofsk (55); Principal Street of Post Korsakofsk on the Island of Sahalin [Sakhalin] (30); Arrival of the mail on Dog Teams (206); Mail Team and P[ost] O[ffice] (110); A Shop at Mihailofsk (102); Bazaar at Derbin on the Island of Sahalin [Sakhalin] (62); Graves of political convicts (92); Contributions for the convicts (63); Skating Rink at Post Alexandrofsk (89); Skating Rink at Post Alexandrofsk (29); Winter view at Post Alexandrotsk (52); Church at Derbinsk (99); Principal Street of Post Alexandrofsk (13); Inside view of Church at Derbinski on Sahalin [Sakhalin] (38); Gilak Children (119); Gilak Old Men (95); Gilak Woman (201); Woman of the Tungus tribe (241); Tungus on the Island of Sahalin [Sakhalin] (238); Convict workmen at Post Dooe (49); Taking the coal from the Diev Coal Shafts (276); Vladimer [Vladimir] Coal Shaft (46); Children of the Rikovsk School (205); Selling in Winter of Products (91); Bread vendors at Post Alexanerofsk [Alexandrovsk] (90); Arrival of the winter mail (3); Coal Shaft at Post Dooe (44); Water carriers for the prison for Chained Prisoners (21); Convict working for himself (197); Repairing a beached lighter at Post Dooe (51); Convicts at work (26); Wharf of the Diew Coal Mines (272); Medical conference at Post Alexandrofsk (114); Contributions for the convicts (63); Alexandrofsk Light-house (130); Taraisk Bridge (142); View of P. Alexandrofsk from the grave yard (24); View of Post Alexandrofsk (56); Violent Chinese Prisoners (211); "Tungus" on the Island of Sahalin [Sakhalin] (198); Woman Convict (164); Statue of Native woman (234); Repairing a beached lighter at Post Dooe (51); Panarama [panorama] of Post Alexandrofsk (229); The Vladimer [Vladimir] Coal Shaft (45); Log carriers on Sahalin [Sakhalin] (71); Church at Derbinsk (99); Wharf at Alexandrofsk (15); Inside view of Church at Derbinski on Sahalin [Sakhalin] (38); Entrance to the Mionchinski Coal Lands (207); Light House "Krilyen" on the Island of Sahalin [Sakhalin] (27); View of Post Alexandrofsk \& the hospital (101); View of the Bazaar at Post Alexandrofsk (203); View of Post Alexandrofsk (56); Water fall 
between Diew and Alexandrofsk (134); Herder (120); Hospital Street of Post Alexandrofsk (23); Children's School, Kosakofsk (5); A collection in the Museum at Post Alexandrofsk (226); Arrival of the mail on Dog Teams (206); Light House "Krilyen" on the Island of Sahalin [Sakhalin] (27); Blessing the waters (250); Gilak Hut (247); Winter Expedition on the Island of Sahalin [Sakhalin] (111); Gilak (87); Gilak in winter Costumes; Gilak Woman (201); Gilak Woman and Child (235); Washing gold at the Nikolaevsk. Mines in Siberia; A collection of Woods \& Minerals in the Museum at Post Alexandrofsk (226); Setting out of the Post (210); Arrival of the winter mail (3); Skating Rink at Post Alexandrofsk (89); Skating Rink at Post Alexandrofsk (29); Type of the life sentence convict (172); Wielder of the 'knout' (105); Convict working for himself (197); Working convicts at dinner (153); Bread Seller of P. Alexandrofsk (28); View of Post Alexandrofsk (18); Bridge across the river 'Alexandrofsk' (60); View of the village of Mihailovsk; Eremeeff, a convict maltreater of Boys (36); Convicts [Convict] (155); Gusel Luborn' a convict (154); Type of a life sentence convict (175); A young Gilak of the Island of Sahalin [Sakhalin] (6); Gilak priest (96); Gilak Poet (8); Gilak in the Museum (237); Articles in the Museum at Post Alexandrofsk (227); Native of Kamchatka (233); Native Woman (240); Drying fish (76); Family of Gilaks (20); Monument [to Count Murav'ev-Amurskii]; Museum (227); Museum (236); Convict workmen at Post Dooe (49); Outside view of the Vladimer [Vladimir] Coal Shafts (218); View of Coal Shafts, 20 versts from Post Alexandrofsk (47); Convicts at work (26); Fishing Station (235); Gilaks at home (234); Gilaks (166); Gilak children (110); Convicts on the road at Post Alexandrofsk (77); Coal Shaft at Post Dooe (44); Tungus on the Island of Sahalin [Sakhalin] (238); Museum at Post Alexandrofsk (231); Street in Rikovsk (140); Gate of the stockade at Alexandrofsk; Outside the Prisons (240); Outside view of the Vladimrr [Vladimir] Coal Mines (216); Police Station in Post Alexandrofsk (219); Convicts carrying beam for lighter (67); A party of chained convicts going to work (82); Group of Convicts (239); A father killer (179); Naumoff, an exconvict who has received 800 blows of the knout (73); Water carriers for the prison for chained prisoners (21); A party of chained convicts going to work (82); Sea Shore dwelling of Photographer Budogionc near Post Alexandrofsk (169).

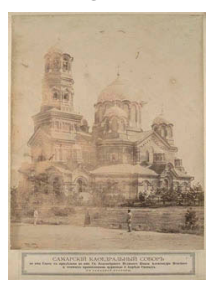

Samarskii kafedral'nyi sobor vo imia Spasa, s pridielami vo imia Sv. Blagoviernago Velikago Kniazia Aleksandra Nevskago i chtimykh pravoslavnoiu tserkov'iu 4 Aprielia Sviatykh. [Russia, 190-?]. 9 photographs, 36 x $44 \mathrm{~cm}$. Caption title. Each photo stamped: Vasil'ev, apparently name of photographer. The original was in a metal cornered portfolio with metal clasp, silver crown and monogram "M.P." Metropolitan New York Library Council, 2008. Slavic Recon Project. Cathedrals - Russia - Samara. Samarskii kafedralnyi sobor (Samara, Russia). Vasilev. Aksakov, I.S. Slav. Reserve (Photo) (Samarskii kafedralnyi sobor) SLV 111 Samarskii kafedral'nyi sobor vo imia Spasa, s pridielami vo 
imia Sv. Blagoviernago Velikago kniazia Aleksandra Nevskago i chtimykh pravoslavnoi tserkov'iu 4 Aprielia Sviatykh (s zapadnoi storony); Samarskii kafedral'nii sobor vo imia Spasa, s pridielami vo imia Sv. Blagoviernago, Velikago kniazia Aleksandra Nevskago i chtimykh pravoslavnoi tserkov'iu 4 aprielia sviatykh (s vostochnoi storony); Vnesennye $\mathrm{v}$ kafedral'nyi sobor brachnye vientsy, soglasno postanovleniia chrezvychainago Samarskago Gubernskago Zemskago Sobraniia, 27 Oktiabria 1891 g., v oznamenovanie dvadtsati-piati lietiia brakosochetaniia Ikh Imperatorskikh Velichestv; Vnesennaia v kafedral'nyi sobor neugasimaia lampada, sooruzhennaia, po postanovleniiu Samarskoi Gorodskoi Dumy 21 Oktiabria 1888 g., v pamiat' sobytiia 17 Oktiabria 1888 g.; Vnesennoe v kafedral'nyi sobor, po postanovleniiu Samarskoi Gorodskoi Dumy 7 Fevralia 1886 g., Sv Evangelie, sooruzhimoe $\mathrm{v}$ uviekoviechenie pamiati ob I. S. Aksakove-propoviednikie idei splocheniia slavianstva (nizhniaia doska); Vnesennoe v kafedral'nyi sobor, po postanovleniiu Samarskoi Gorodskoi Dumy 7 Fevralia 1886 g., Sv. Evangelie, sooruzhimoe $\mathrm{v}$ uviekoviechenie pamiati ob I. S. Aksakoviepropoviednikie idei splocheniia slavianstva (verkhniaia doska); Vnutrennii vid Samarskago Kafedral'nago Sobora. Lievyi pridiel vo imia chtimykh pravoslavnoiu tserkov'iu 4 Aprielia Sviatykh; Vnutrennii vid Samarskago Kafedral'nago Sobora. Pravyi pridiel vo imia Sv. Blagoviernago Velikago Kniazia Aleksandra Nevskago; Vnutrennii vid Samarskago Kafedral'nago Sobora. Glavnyi prestol vo imia Spasa.

Sevastopol' $v$ 1855-1856 g.: 25 fototipicheskikh snimkov $s$ riedkago fotograficheskago al'boma, izdali $P$. Kharitonenko i N. Golubov. Moscow: P. Kharitonenko i ko., 1893. 25 leaves of plates; 1 p. of text; $44 \times 35 \mathrm{~cm}$. Each plate reads: "Fototipiia Sherer, Nabgol'ts i Ko. v Moskvie." Crimean War, 1853-1856. Sevastopol (Ukraine) - Pictorial works. Slav. Reserve (Photo) 99-1695 SLV 112 [Cover title]; Korabel'naia bukhta i Pavlovskii mysok; Odno iz Sevastopol'skikh zdanii, zaniatoe Frantsuzami; Angliiskii lager' pod Sevastopolem; Lager' 97go Angliiskago polka pod Sevastopolem; Doki i Korabel'naia bukhta; Sevastopol'skaia ulitsa; Batareia na Malakhovom kurganie; Fediukhinyi vysoty i Traktirnyi most; Vnutrennost' 3-go bastiona; Vid na Iuzhnuiu bukhtu so storony 3-go bastiona; Vid na Iuzhnuiu bukhtu so storony 4-go bastiona; Nepriiatel'skiia batarei pered Malakhovym; Vid s Malakhova na Sievernuiu bukhtu i na Korabel'nuiu; Blindazh 3-go bastiona; Sevastopol'skaia batareia; Konstantinovskii fort; Sevastopol'skaia ulitsa; 21aia Angliiskaia batareia pod Sevastopolem; Biblioteka; Vid iz goroda (s Iuzhnoi) na Korabel'nuiu; Vid s Malakhova na Iuzhnuiu i Sievernuiu bukhty; Vid s Sievernoi storony na Sevastopol'; Vid so storony Malakhova na Iuzhnuiu storonu; Bashnia Malakhova kurgana; Kazarmy. 
St. Petersbourg. [St. Petersburg]: Velten, [1870?] 1 album (24 photographs), $33 \times 25 \mathrm{~cm}$. Cover title. Photographs mounted on cards.

Original publisher's cloth with gilt decorations and embossed lettering on upper cover. Purchased from Bernard J Shapero Rare Books, 2007. Saint Petersburg (Russia). Photography-Russia-19th century. Slav. Reserve (Photo) 07-6536 SLV 116

1. [Monument to Peter the Great from Ekaterina II]; 2. [The Admiralty and Neva embankment]; 3. [Nevskii prospect and the Admiralty]; 4. [Peter and Paul Fortress]; 5. [Stock Exchange]; 6. [The Small Hermitage]; 7. [Winter Palace and Alexander column]; 8. [Palace Square and Alexander column]; 9. [Kazan Cathedral]; 10. [St. Issac's Cathedral]; 11. [A Protestant Church]; 12. [Monument to Nicholas I]; 13. [Chapel in the Summer Garden]; 14. [Monument to Mars in the Field of Mars]; 15. [Sculpture of "Taming the Horses" on Anichkov Bridge with passersby]; 16. [Sculpture of "Taming the Horses" on Anichkov Bridge]; 17. [Sculpture of "Taming the Horses" on Anichkov Bridge]; 18. [Sculpture of "Taming the Horses" on Anichkov Bridge]; 19. [Neva embankment]; 20. [Nevskii Prospect]; 21. [House of Peter the Great in the Summer Garden]; 22. [Marble Palace]; 23. [Engineers' Castle]; 24. [Trinity Cathedral and Column of Glory].

Suzdal'skie monastyri: Spaso-Evfimievskii, Pokrovskii, [Rispolozhenskii] Prepodobenskii; al'bom vidov i snimkov drevnostei i dostoprimechatel'nostei, khraniashchikhsia $v$ ikh riznitsakh. [Russia?: s.n., 18-] 68 mounted photographs,

$33 \times 43 \mathrm{~cm}$.

With captions in ms., originally in portfolio. Each photograph with stamp reading: "Fotografiia voskresen. monast. Ierod. Diodora." With bookplate of Alexander III, Emperor of Russia. Title from the shelflist card.

Metropolitan New York Library Council, 2008. Slavic Recon Project. Spaso-Evfimiev monastyr' (Suzdal', Russia). Monasteries, Orthodox Eastern - Russia (Federation) - Suzdal. Suzdal (Russia). Slav. Reserve (Photo) (Suzdalskie monastyri) SLV 118 Obshchii vid Suzdalia s Vladimirskoi dorogi; Obshchii vid Suzdal'skago Spaso Evfimieva Monastyria; Viezdnaia bashnia v Spaso Evfimievom Monastyre; Viezdnaia bashnia v Spaso Evfimievom Monastyre; Uglovaia bashnia s chast'iu ogrady v Suzdal'skom Spaso Evfimievom Monastyrie; Zvonitsa Suzdal'skago Spaso Evfimieva Monastyria; Grobnitsa Kniazia Dmitriia Mikhailovicha Pozharskago v Suzdal'skom Spaso Evfimievom Monastyrie; Zapis' na Evangeliie, ruki kniazia D. M. Pozharskago; Raka Prepodobnago Evfimiia Suzdal'skago; Ikony nad rakoiu Prepodobnago Evfimiia; Shapochka Prepodobnago Evfimiia No. 1 (Suzdal'); Shapochka Prepodobnago Evfimiia No. 2 (Suzdal'); Tsarskiia dveri v riznitsie Suzdal'skago Spaso Evfimieva Monastyria; Evangelie kniazia Dmitriia Mikhailovicha Pozharskago 
v riznitsie Suzdal'skago Spaso Evfimieva Monastyria; Naprestol'nyi krest v riznitsie Suzdal'skago Spaso Evfimieva Monastyria; Darokhranitel'nitsa kniazia Dolgorukova v riznitse Spaso Evfimieva Monastyria (Suzdal'); Kadilo v riznitsie Spaso Evfimieva Monastyria (Suzdal'); Potir, pozhertvovannyi Nogtevym, v riznitsie Spaso Evfimieva Monastyria; Obshchii vid Suzdal'skago Pokrovskago Monastyria. Miesto zatocheniia Tsaritsy Evdokhii Feodorovny; Tsarskie vrata v pridiele Smolenskoi Bozh'ei Materi v Pokrovskom Monastyre; Chast' dverei riznitsy Pokrovskago Monastyria; Ikona Gruzinskoi Bozh'ei Materi v Suzdal'skom Pokrovskom Monastyrie. Vklad Tsaria Ivana Vasil'evicha Groznago; Zolotoi skladen' s Evangeliem v Suzdal'skom Pokrovskom Monastyrie; Obraz Sv. Nikolaia Chudotvortsa v Suzdal'skom Pokrovskom Monastyre, chto u tsarskikh vrat (Suzdal'); Obraz Sv. Nikolaia Chudotvortsa v Suzdal'skom Pokrovskom Monastyrie (Suzdal'); Zolotoe kadilo v riznitsie Pokrovskago Monastyria; Iz riznitsy Pokrovskago Monastyria, No. 1; Iz riznitsy Pokrovskago Monastyria, No. 2; Iz riznitsy Pokrovskago Monastyria, No. 3; Iz riznitsy Pokrovskago Monastyria, No. 4; Iz riznitsy Pokrovskago Monastyria, No. 5; Iz riznitsy Pokrovskago Monastyria, No. 6; Iz riznitsy Pokrovskago Monastyria, No. 7; Iz riznitsy Pokrovskago Monastyria, No. 8; Iz riznitsy Pokrovskago Monastyria, No. 9; Iz riznitsy Pokrovskago Monastyria, No. 10; Iz riznitsy Pokrovskago Monastyria, No. 11; Obshchii vid Suzdal'skago Prepodobenskago Monastyria; Sviatye vorota v Prepodobenskom Monastyrie; Troitskaia tserkov' v Prepodobenskom Monastyrie; Vkhodnyia dveri v Troitskuiu tserkov' Suzdal'skago Prepodobenskago Monastyria; Okna Troitskoi Tserkvi Prepodobenskago Monastyria (Suzdal'); Izrashchy v kolokol'ne Troitskoi tserkvi Prepodobenskago Monastyria; Kadilo v riznitsie Prepodobenskago Monastyria; Suzdal'skii Sobor; Vkhodnyia dveri v Suzdal'skii Sobor; Arkhiereiskii dom s kolokol'nei Suzdal'skago Sobora; Sievernyia dveri ikonostasa Suzdal'skago Sobora; Ikonostas Suzdal'skago Sobora; Vodosvetnaia chasha sobornoi riznitsy (Suzdal'); Afonskii krest Suzdal'skago sobora s litsevoi storony; Afonskii krest Suzdal'skago sobora s obratnoi storony; Fonar' Suzdal'skago sobora; Kapiteli nad vkhodnymi dveriami Suzdal'skago sobora; Shater krytii goluboi cherepitsei nad papert'iu Suzdal'skago sobora; Kolonki i karnizy na narushnoi stiene Suzdal'skago sobora; Darokhranitel'nitsa $\mathrm{v}$ riznitsie Suzdal'skago sobora; Stakan finiftianoi v riznitsie Suzdal'skago sobora; Dom Suzdal'skago dukhovnago uchilishcha; Okno nizhniago etazha Suzdal'skago uchilishcha; Izrashchovaia pech' v Suzdal'skom dukhovnom uchilishche; Chast' pechi v Suzdal'skom dukhovnom uchilishche; Izrashchovaia pech' $\mathrm{v}$ Suzdal'skom dukhovnom uchilishche; Vorota Tserkvi Vkhoda Gospodnia v Suzdalie; Tserkov' Sv. Nikolaia Chudotvortsa v Suzdalie; Vkhod v tserkov' S. Ioanna Predtechi v Suzdalie; Krest nad tserkov'iu Kazanskoi Bozh'ei Materi (Suzdal'); Vorota tserkvi Voskreseniia Khristova v Suzdalie. 
Tipy naselenii Penzenskoi gubernii/sniaty s natury dieistvitel'nym chlenom Penzenskago Gubernskago Statisticheskago Komiteta Kollezhskim Sekretarem Nikolaem Orlovym. Penza, 1862. 1 v., 12 mounted photographs (col.), $26 \times 31 \mathrm{~cm}$. Title page in ms., with coat of arms of the government of Penza at head of title; captions in ms. Twelve hand-colored photographs. Master Negative ${ }^{\star} \mathrm{ZZ}$ 30916 Orlov, Nikolai. Costume-Russia. Penza (Russia) Mordvins. Slav. Reserve (Photo) 93-3675 SLV 119 [Title page]; Russkie; Russkie; Russkie; Russkie; Russkie; Russkie; Russkie; Selo Rybkino Krasnoslobodskago uiezda (Mordva moksha); Mordva; Mordva; Mordva; Tatary.

[Troitskaia Lavra i Prep. Sergii Radonezhskii; al'bom fotografii] ca. 1880. 26 mounted photographs; $39 \mathrm{~cm}$. ${ }^{\star} \mathrm{ZQ}-1006$ "Bound album of 26 albumen prints $( \pm 8 \times 101 / 2$ "), most with captions pasted under the images. Some with elaborate details. Title and a list of contents appear on typewritten pages pasted on the first blank pages of the album. Not contemporary, as is red cover. Album documents the holy treasures of the monastery, mainly the objects having belonged to St. Serge of Radonej, and several well-known icons. It includes: views of monastery and church; views of monastery and church; the reliquary of St. Serge; icon of Trinity and Icon of Virgin and Child; icon of St. Serge, having worked miraculous cures during cholera epidemic of 1848; Virgin and Child, Smolensk; St. Nicholas; Gospel having belonged to St. Serge, 14th century (no. 1); prayerbook, written by St. Nicholas, used by St. Serge, 1381 (no. 15); wooden vessels used by St. Serge and St. Nikon, his disciple (nos. 5 \& 6); chausubles of St. Serge (nos. 7 \& 8); other clothes of St. Serge; sandals of St. Serge; icon of the Vision of the Virgin, above south door of altar of Church of the Trinity, paraded in wars (Poland, Sweden, Napoleonic, Crimea); icons of Virgin Mary, savior (11th c.), Jesus, Virgin, St. John the Baptist, "Panagiya." Important document for the aspect and condition of the treasures of the Monastery. The photos themselves are copy prints (see dimensions written on original photos being reproduced)" (Pierre Apraxine). DOE Title II-C "Russian Illustrated Books and Photographs" Project.

Troitse-Sergieva lavra. Icons, Russian. Church vestments-Russia. Sergii, Radonezhskii, Saint, ca. 1314-1391 or 2. Slav Reserve (Photo) (Troitskaia lavra) SLV 122 [Cover title]; Vid Sviato-Troitse-Sergievoi Lavry; Tserkov'; Izobrazhenie raki, v kotoroi pokoiatsia Sv: moshchi Pr. Sergiia; Ikona; Ikona; Ikona Prepod. Sergiia v tserkvi Sv. Velikomuchenits Varvary i Anastasii; Ikona Bogoroditsy Smolenskiia No. 3. keleinaia Pr. Sergiia; Ikona Sviatitelia Nikolaia Chudotvortsa No. 4. keleinaia Pr. Sergiia; Evangelie No. 1. Prepodobnago Sergiia na pergamentie XIV vieka; Sluzhebnik No. 15. pisannyi v 1381 godu na pergamentie rukoi Prepodobnago Nikona i upotrebliavshiisia Prepodobnym Sergiem; Dereviannye sosudy No. 5. 
upotrebliavshiesia v sluzhenii Prepodobnym Sergiem; Dereviannye sosudy No. 6. upotrebliavshiesia v sluzhenii Prepodobnym Nikonom, uchenikom Pr. Sergiia i preemnikom Ego; Riza Prepodobnago Sergiia No. 7 krasheninnaia; Riza No. 8. i Epitrakhil' No. 10, Prepodobnago Sergiia bumazhnoi kamki; Skhima Prepodobnago Sergiia sukonnaia temnokarichnevaia; Skhima Prepodobnago Sergiia No. 14. iz chernago mukhoiara; Poruchi Prepodobnago Sergiia No. 11. sinei ob'ari; Sandalii Prepodobnago Sergiia No. 18. kozhannyia, 30 liet byvshiia na nogakh ego vo grobie; Lozhka i nozh'; Ikona Vidieniia Bozhiei Materi nad iuzhnoi dver'iu altaria Troitskago Sobora, No. 7. iz dski ot dereviannoi raki Prepod. Sergiia; Ikona Presv. Bogoroditsy Ierusalimskiia No. 11 nad Tsarskimi vratami Tserkvi Pr. Nikona ot vremen Apostol'skikh; Ikona Nerukotvorennago Obraza Spasiteleva No. 1. drevniaia; Ikona De-Iisus, No. 47 na trekh doskakh; Ikona;

Ikona; Panagiia, No.9. na agatie s nerukotvorennym izobrazheniem moliashchagosia pred Raspiatiem (s podpis'iu).

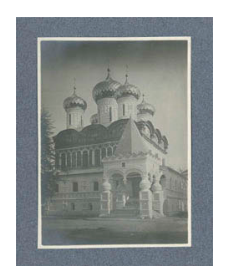

Troitskii sobor $v$ Ipat'evskom monastyrie: Remont, 19111913 g. Russia: s.n, 191-? 26 mounted photographs on 20 leaves, $28 \times 35 \mathrm{~cm}$. Issued in slip case. Cover title. Slavic Recon Project. Metropolitan New York Library Council, 2008. Troitskii sobor Sviato-Troitskogo Ipatevskogo monastyria (Kostroma, Kostromskaia oblast, Russia) - Conservation and restoration. Cathedrals - Conservation and restoration - Russia (Federation) -Kostroma (Kostromskaia oblast). Slav. Reserve (Photo) (Troitskii sobor v Ipatievskom monastyrie) SLV 123 [Cover title]; Tsar' Mikhail Feodorovich, freska na stene sobora; Tsar' Mikhail Feodorovich, freska na stene sobora; Obshchii vid sobora do i poslie restavratsii [left side]; Obshchii vid sobora do i poslie restavratsii [right side]; Sobor s sieverovostochnago ugla do restavratsii; Sobor s sievero-vostochnago ugla posle restavratsii; Barabany sobora do i poslie remonta [above]; Barabany sobora do i poslie remonta [below]; Restavratsiia perekrytiia pridela prepodobnago Mikhaila Maleina [above]; Restavratsiia perekrytiia pridiela prepodobnago Mikhaila Maleina [Below]; Naruzhnaia sievernaia stiena sobora do remonta; Naruzhnaia iuzhnaia stiena sobora do remonta; Detal' treshchiny iuzhnoi stieny sobora; Vkhod v podval sobora do i poslie restavratsii [above]; Vkhod $\mathrm{v}$ podval sobora do i posle restavratsii [below]; Remont tsokolei sobora [above]; Remont tsokolei sobora [below]; Treshchiny $\mathrm{v}$ svodakh gallereia [above]; Treshchiny v svodakh gallereia [below]; Sievernyi portal sobora do remonta; Sievernaia gallereia sobora vo vremia issliedovanii; Sievernaia gallereia sobora poslie restavratsii; Zapadnaia gallereia sobora poslie restavratsii; Detal' treshchiny na freskakh iuzhnoi stieny sobora; Freska sievernoi stieny sobora do restavratsii (Pesn' pesnei); Freska sievernoi stieny sobora posle restavratsii (Pesn' pesnei). 


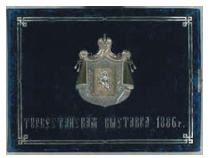

Turkestanskaia vystavka 1886 g. Tashkent, 1886. 35 mounted photographs, $61 \times 45 \mathrm{~cm}$. Markings: "Wormer May 3, 1945." Bookplate of Alexander III, Emperor of Russia (1845-1894). Binding title. Binding, contemporary, of blue velvet, with white silk doublure, full gilt edges, raised metal border, letters and coat of arms on with bookplate of Emperor Aleksander III. All plates read: "Vid Turkestanskoi vystavki 1886 goda. Snimal s natury fotograf V. Kozlovskii." Slavic Recon Project. Metropolitan New York Library Council, 2008. Exhibitions - Kazakhstan. Industries - Asia, Central - Exhibitions. Slav. Reserve (Photo) (Turkestanskaia vystavka 1886 g) SLV 124 [Cover title]; [Pavilion with portrait of Alexander III]; [Pavilion at the center of exhibition square]; [A section of the Tashkent stand]; [A section of the Samarkand stand]; [The stands of Makhamedova and Abdullinova factory]; [A stand of Diurshmidt Brothers]; [Pavilion of Diurshmidt Brothers]; [Military brassband in the exhibition]; [Military brass-band and people]; [Stand of I. Krauze]; [Rock crystal]; [The stand of Diurshmidt Brothers]; [Pavilion for Filatov's factory]; [Pavilion for Filatov's factory]; [Pavilion for N.I. Ivanov's factory]; [Pavilion for Gromova's factory];[Pavilion for Lakhtin's factory]; [Pavilion for unknown alcoholic beverage factory]; [Pavilion for mining production of D.P. Petrov]; [Pavilion, for the tannery and interalia of A.N. Vul'fzona]; [Pavilions for unknown factories]; [Pavilion for tobacco factory of K.V. Bubnov]; [Pavilion for linen and cotton manufacture of D.G. Donskoi]; [Pavilion for Gille i Ditrikh]; [Stand for horse breeders of N.I. Ivanov]; [N.I. Ivanov's stand]; [Stand for horse stables of Said-Azim-Baev]; [Stand for Silk]; [M. Deich's stand]; [Stand for Metchikov]; [Pavilion for A.P. Blinovskii's factory]; [Pavilion for tannery of I.D. Tezikov]; [Pavilion for unknown factory]; [Pavilion for tannery of M.A. Mir-Aiupov]; [Pavilion for experiment center of Akrym Askarov].

Turkestanskaia vystavka 1890 g. Tashkent: Fotografiia St. Nikolaia, [1890]. 45 mounted Photographs, $32 \times 48 \mathrm{~cm}$. Caption title. "Sobstvennost' izdatelia." The album presented to Emperor Alexander III. Originally in portfolio. A letter from the Hammer Galeries, New York dated June 24, 1949 reads in part: “...These photographs were contained in an album presented to Alexander III which was destroyed. The portfolio bore the inventory label of the Winter Palace, item \#13, bookcase \#13, shelf \#1." Slavic Recon Project. Metropolitan New York Library Council, 2008. Industries - Asia, Central Exhibitions. Exhibitions - Kazakhstan. Slav. Reserve (Photo) (Turkestanskaia vystavka 1890 g) SLV 125 Obshchii vid pri vkhodie v glavnyia voroty; Glavnyia vkhodnyia vorota; Lietnyi teatr Tashkentskago obshchestva liubitelei dramaticheskago iskusstva; Pavil'on dlia tuzemnago strunnago orkestra; Khlopkovye tiuki raznykh zavodov; Orudiia firmy "Rabotnik"; Proba orudii zemledeliia; Pavil'on dlia tuzmenago dukhovago orkestra; Pavil'on dlia 
voennago orkestra; Pavil'on dlia Bukharskago otdiela; Pavil'on dlia Khivinskago otdiela; Pavil'on dlia otdiela I: "polevodstvo i sel'skaia promyshlennost"”; Plantatsiia pri otdiele I: "polevodstvo i sel'skaia promyshlennost"; Orudiia firmy "Rabotnik"; Pavil'on dlia otdiela II: "sadovodstvo, vinogradarstvo i vinodielie"; Pavil'on dlia otdiela III: "shelkovodstvo"; Bashnia iz kokonov I. Aloizi; Pavil'on dlia otdiela III: "khlopkovodstvo"; Pavil'on khlopkovykh plantatsii i khlopko-ochistitel'nago zavoda G. M. Bieliakova bliz g. Tashkenta; Pavil'on dlia otdiela IV: "Ptitsevodstvo"; Pavil'on dlia otdiela IV: "Konevodstvo"; Pavil'on-koniushnia Kommertsii Sovetnika N. I. Ivanova; Pavil'on dlia otdiela V: "lesovodstvo"; Pavil'on dlia otdiela VI: "okhota"; Pavil'on dlia otdiela VI: "rybolovstvo"; Obshchii vid glavnoi ploshchadi ot kustarnago otdiela; Pavil'on dlia otdiela VII: "kustarnyi". (panorama); Pavil'on dlia otdiela VIII: "gornyi"; Pavil'on dlia otdiela IX: "nauchnykh trudov po Azii"; Pavil'on dlia otdiela X: "voenno-istoricheskii"; Kolokol, vesom v 570 p., otlityi v g. Tashkentie dlia sobora Turkestanskikh voisk; Piramida iz voennykh trofei; Figura Russkago voina, vodruzhaiushchago znamia; Pavil'on Tashkentskoi gorodskoi tiurmy; Pavil'on tuzemnaia kukhnia; Pavil'on Zakaspiiskoi zhelieznoi dorogi; Pavil'on Kommertsii Sovetnika N. I. Ivanova; Pavil'on Tashkentskago kuptsa Metrikova; Pavil'on Tashkentskago sarta kuptsa Akrym Askarova; Pavil'on D. L. Filatova; Pavil'on firmy "brat'ia Pervushiny"; Pavil'on torgovago doma "brat'ia Iaushevy"; Pavil'on kozhevennago zavoda I. D. Tezikova v g. Tashkente; Pavil'on Torgovo-Promyshlennago Tovarishchestva Iaroslavskoi Bol'shoi Manufaktury; Pavil'on-restoran Tashkentskago kuptsa F. G. Gavrilova.

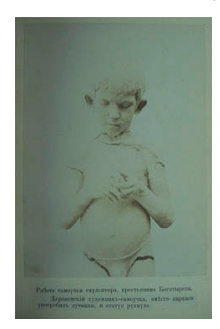

Tverskoi muzei 13 iunia 1892 goda. Poseshchenie muzeia Ego Imperatorskom Vysochestvom Velikim Kniazem Vladimirom Aleksandrovichem. Tver: Tip. Gubernskago pravleniia, 1892. 40 p., 20 leaves of plates, $26 \mathrm{~cm}$. MN ${ }^{*} Z Z-31653 \&{ }^{\star} Z$ Q-956 DOE Title II-C "Russian Illustrated Books and Photographs" Project. Museums-Russia-Tverskaia guberniia. Museums-Russia-Tver. Slav. Reserve (Photo) (Tverskoi muzei) SLV 126

1. Vnutrennii vid Muzeia; 2. Vnutrennii vid Muzeia; 3. Kamennayia orudiia; 4. Izrazets; 5. Kamen's priamougol'nymi chetyreugol'nikami i s nadpis'iu "Stepan" - iz Biezhetskago uiezda. (Tversk. Muzei); 6. Nadgrobnyi pamiatnik 1499 goda, Afanasiia Ivanova syna Ostrozheva. 1/6; 7. Sterzhenskii krest 1133 goda; 8. Lopastitskii krest; 9. [Sobranie krestov]; 10. [Sobranie krestov]; 11. Zmeeviki; 12. Emalevaia korobochka; 13. Rukoiat' iz mamontovoi kosti; 14. Pechat' Novotorzhskago namiestnika i pechat' Sibirskago prikaza sobolina; 15. Kostianaia grebenka; 16. Ser'gi; 17. Ornamenty i perstni; 18. [Monetnaia kollektsiia Tverskogo Muzeia]; 19. [Monetnaia kollektsiia Tverskogo Muzeia]; 20. [Monetnaia kollektsiia Tverskogo Muzeia]; 21. Rabota samouchki skul'ptora, krest'ianina 
Bogatyreva. Derevenskii khudozhnik-samouchka, vmesto karkasa upotrebil luchinki, i statuia rukhnula.

Vidy g. Koly. [S.l.: Ia. Leitsinger, ca. 1880] 24 mounted photographs on 9 leaves, $26 \times 33 \mathrm{~cm}$. "The album is the collection of 19 book pages of a disbound album (no cover), consisting of 24 albumen prints in two sizes... on mounts with gilded frames. Small prints are two to a mount, with pasted printed titles and the signature of the photographer imprinted on the lower right corner: Ya. Leitsinger. Views of the city of Kola [in Murmanskaya oblast'], sites of the Pechenskii Monastery, factories, camps, churches, and hospitals of various camps. Harbor installations; 2 group portraits of Lapps. Good documentation on rather primitive installations on the border with Norway. Prints of fine quality. A very good image of a Lapp family. Good condition of prints and mounts. The date is suggested by the appearance of the prints." (Pierre Apraxine) Pechenskii monastyr. Kola Peninsula (Russia). Sami (European people)-Russia. Slav Reserve (Photo) (Vidy g. Koly) SLV 129 Kola; Tserkov' gdie moshchi pr. Trifona Pechenskago; Gora gdie zhil pr. Trifon Pechenskii; Pechenskii monastyr' na granitsie Norvegii; Faktoriia kuptsa Savina; Factoriia kolon. v st. Tsyp-Navolok; Stanovishche Gavrilovo; Stanovishche Shel'pina; Stanovishche Teriberka; Kitolovnyi zavod Ara-guba; Stanovishche Eretiki; Lopary; Kol'skiia lopari lietom; Promyslovyia izby [above]; Tserkov' na miestie razzorennago shvedami monastyria [below]; Shkuna; Stan. Korabel'naia guba [below]; Bol'nitsa v st. Tsyp-Navolok [above]; Bol'nitsa v st. Teriberka [below]; Stanovishche Litsa [above]; Bol'nitsa v st. Gavrilova [below]; Nazhivka peshchanki (dlia lovli treski) [above]; Sutka treski [below]; [Priest and Crew on a Ship].

Vidy g. Novgoroda i ego okrestnostei. S.l.: 1883. 24 mounted photographs, $23 \times 35 \mathrm{~cm}$. " 24 albumen prints mounted on boards with printed gilt titles, approx. 12 1/2 x 8 1/2"; churches, monasteries, remarkable buildings (residence of Governor, House of the Nobility, Police headquarters), ruins, monuments, walls, Palace of Yaroslavl', etc., a panorama made of 15 photos mounted on 10 boards: 'General view of Novgorod,' showing city at a 360 degree angle. Album now disbound and boxed. Documentary interest. Prints tend towards yellowing." (Pierre Apraxine). DOE Title II-C "Russian Illustrated Books and Photographs” Project. Master Negative: ${ }^{\star} Z Z-30651$. Novgorod (Russia). Architecture-Russia -Novgorod. Slav. Reserve (Photo) (Vidy g. Novgoroda) SLV 130 Cover: Vidy g. Novgoroda i ego okrestnostei, 25 iiulia 1883 g.; Panorama; Bielaia Bashnia; Muzei i Biblioteka; Korsunskiia Voroty v Sofiiskom Soborie; Tserkov' Spasa-Nereditsy; Selo RiurikovoGorodishche; Pamiatnik Tysiachalietiia s iugo-vostochnoi storony; Nikolaevskii Priiut; 1-ia Sofiiskaia politseiskaia chast' i pozharnyi oboz; Antoniev Monastyr'; Dom Dvorianstva, gdie sokhraniaetsia Ekaterininskaia 
lodka; Znamenskii Sobor; Dom Nachal'nika Gubernii; Tserkov' Rozhdestva na Molotkovie; Sofiiskii Sobor; Pamiatnik Dvorianstva 1812 g.; Mitropolichii Dom; Tserkov' Spasa; Domovaia tserkov' Sv. Ioanna Arkhiepiskopa; Zvonitsa Sofiiskago Sobora; Dvorianskoe Sobranie; Efim'evskaia Bashnia; Pamiatnik Tysiachalietiia Rossii; Pytochnaia Bashnia; Bashnia Iaroslava i Ego Dvorishche; Obshchii Vid Novgoroda; Obshchii Vid Novgoroda; Obshchii Vid Novgoroda; Obshchii Vid Novgoroda; Obshchii Vid Novgoroda; Obshchii Vid Novgoroda; Obshchii Vid Novgoroda.

Vidy g. Sol'vychegodska i ego dostoprimiechatel'nostei. Vologda: Fotografiia Baranieva, [n.d.] 21 mounted photographs, $33 \mathrm{x}$ $24 \mathrm{~cm}$. "Album now disbound and boxed with cloth cover with gilt letters: E.I.V.G.V.K.V.A. (for Vladimir Alexandrovich). Views of the city of Sol'vychegodsk, and its remarkable sites. 21 albumen prints mounted on boards of different sizes. 9 prints ( $\pm 6 \times 8$ ") mounted on larger boards ( $91 / 4 \times 123 / 4$ "); 12 prints $( \pm 51 / 2 \times 4$ ") on smaller ones (9 $1 / 4$ x $81 / 2$ "), with printed decorative frames and captions. 'Photography by Baranieev in Vologda' with address and mention of a 'silver medal for contributing to the success of archeology'. All photos numbered 1-19 and above the images by hand in ink in calligraphic script. The numbers refer to the subjects photographed, some recto and verso." City Solvychegodsk is located in Archangel oblast, $18 \mathrm{~km}$ from Kotlas." (Pierre Apraxine). DOE Title II-C "Russian Illustrated Books and Photographs" Project. Master Negative: *ZZ-30648. Orthodox Eastern Church-Russia-Liturgical objects. Solvychegodsk (Russia). Church architecture-Russia. Icons, Russian. Church vestments-Russia. Slav Reserve (Photo) (Vidy g. Solvychegodska) SLV 131 [Cover title]; No. 1. Vid tsentral'noi chasti g. Sol'vychegodska s iugo-zapadnoi storony; No.2. Vid Sol'vychegodskago Blagovieshchenskago sobora s sieverozapad. storony; No .3. Obshchii vid tsarskikh vrat Sol'vychegodskago Blagovieshchenskago sobora; No. 3. Vid tsarskikh vrat Sol'vychegodskago Blagovieshchenskago sobora; No. 4. Sakkos s Stefana Velikopermskago, szadi; No. 4. Sakkos s Stefana Velikopermskago, speredi; No. 5. Ikona Vsemilostivieshnago Spasa; No. 6. Ikona Presviatoi Bogoroditsy; No. 7 Ikona Presviatoi Bogoroditsy; No. 8. Sosud dlia ladona; No. 8. Sosud dlia kut'i; No. 10. Panagii; No. 11. Lampada dlia sviech; No. 12. Ekipazh Stroganovykh; No. 13. Ekipazh Stroganovykh; No. 14. Vid klirosa sboku; No. 15. Chast' osnovaniia ikonostasa kholodnago khrama; No. 16. Vid Sol'vychegodskago Vvedenskago monastyria s iu. v. storony; No. 17. Vid Sol'vychegodskago Vvedenskago monastyria s iu. v. storony; No. 18. Vid kryl'tsa Sol'vychegodskago Vvedenskago monastyria; No. 19. Krest'ianskiia zhenshchina i dievushka Sol'vychegodskago uiezda. 
Vidy g. Tot'my. Vologda: Fotografiia Baranieeva. 9 mounted photographs, $33 \mathrm{~cm}$. With cloth cover with gilt letters: E.I.V.G. V.K.V.A. (dedication to Grand Duke Vladimir Aleksandrovich. See above listing for work by same photographer, Vidy g. Solvychegodska.) "Small album now disbound and boxed... 9 albumen prints mounted on board with printed decorative frames and captions: 'Photography by Baraniev in Vologda,' with address and mention of a silver medal for contributing to the success of archeology. Views of the city of Tot'ma [city in Totemskii raion in Vologodskaia oblast]. All the photographs are titled above by hand in ink.

3 views of monastery at Tot'ma, 4 views of churches and plazas, seen from above; 2 street views at street level, one of which (no. 9) is outstanding: 'partial view of the marketplace....'

Of documentary and aesthetic interest. 6 rich and delicate prints. Excellent example of city portraiture." (Pierre Apraxine). DOE Title II-C "Russian Illustrated Books and Photographs" Project. ${ }^{*}$ ZQ-1010 Master Negative: ${ }^{*}$ ZZ-30644 Baraneev (photographer) Totma (Russia). Church architectureRussia. Slav. Reserve (Photo) (Vidy g. Tot'ma) SLV 132 E.I.V.G.V.K.V.A. [Ego Imperatorskomu Vysochestvu Gosudariu Velikomu Kniaziu Vladimiru Aleksandrovichu] [Cover title]; Obshchii Vid Totemskago monastyria s vostochnoi storony; Vid chasti Totemskago monastyria s vostochnoi storony; Vid Totemskago monastyria s zapadnoi storony; Vid tserkvi Srieteniia Gospodnia i gostinnogo dvora s kolokol'ny Georgievskoi tserkvi; Vid sobora i sobornoi ulitsy g. Tot'my i chast' torgovoi ploshchadi; Vid na Zeleniu i rieku Sulonu i na torgovyia riady s zapadnom storony g. Tot'my; Obshchii vid g. Tot'my i chast' torgovoi ploshchadi i Georgievskoi tserkvi; Vid uchitel'skoi seminarii g. Tot'my; Vid chasti torgovoi ploshchadi g. Tot'my.

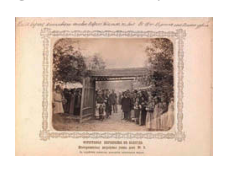

Vidy g. Velikago-Ustiuga. Vologda: Fotografiia Baranieeva, [18-?]. 8 mounted photographs, $26 \times 33 \mathrm{~cm}$. Cover reads "E. I. V. G. V. K. B. A..." Title from original portfolio, which bears the initials of Grand Duke Vladimir Aleksandrovich.

Each mount is marked: "Fotografiia Baranieeva v Vologdie.... Za sodieistvie uspiekham arkheologii serebrannaia medal'." Slavic Recon Project. Metropolitan New York Library Council, 2008. Velikii Ustiug (Russia). Slav. Reserve (Photo) (Vidy g. Velikago Ustiuga) SLV 133 [Cover title]; Snimok s chudotvornoi ikony Veliko-ustiugskago sobora; Vid Kotolovskoi chasovni s bogomol'tsami bliz g. Velikago Ustuga [Ustiuga] v 18-ti verstakh $\mathrm{v}$ znak chiudnago spaseniia ot kamennoi shugi; Vid vorot Kotolovskoi chasovni vo vremia Bogomol'ia, 25go Iunia. V 18-ti verstakh ot Velikago ustiuga; Vid gory Kotolova s chasovni gde vypali pervyia komni, i gde nashli moliashchiimsia Velikago ustiugskago ugodnika Prokopiia Pravednago; Vid naberezhnykh tserkvei g. Velikago-ustiuga s kolokol'ny Ioanna Bogoslova; 
Obshchii Vid g. Velikago Ustiuga s kolokol'ny Ioanna Bogoslova s iugo vostochnoi storony; Obshchii vid g. Velikago Ustiuga s kolokol'ny Ioanna Bogoslova s iuzhnoi storony; Vid gorodishcha v g. Velikom Ustiuge, pervoe nachalo goroda i zakladka khrama.

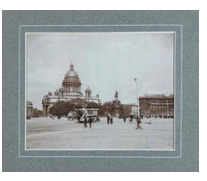

Vidy goroda S. Peterburga. St. Petersburg, 1900? 14 mounted photographs, $34 \times 44 \mathrm{~cm}$. Cover title. Purchased from A. Rabinovich. Saint Petersburg (Russia). Slav. Reserve (Photo) 98-6718 SLV 134 [Cover title]; [Mikhailovskii dvorets]; [Sobor sv. Troitsy i Pamiatnik Slavy]; [Akademia Khudozhestv]; [Isaakievskaia ploshchad']; [Nevskii prospekt]; [Isaakievskii sobor]; [Nikolaevskii Most]; [Chasovnia i Lietnii sad]; [Narvskie triumfal'nye vorota]; [Pamiatnik Petru Velikomu i Isaakievskii sobor]; [Troitskii most]; [Chasovnia u Lietniago sada]; [Glavnago Shtava]; [Khram Voskreseniia Khristova].

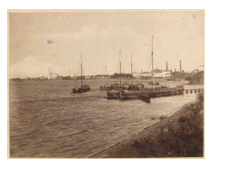

Vidy goroda Shlissel'burga. St. Petersburg: Fotografiia S. Savitskii, [18-]. 4 mounted Photographs, $30 \times 22 \mathrm{~cm}$. Binder's title. Bookplate of Grand Duke Vladimir Aleksandrovich. The front cover of the book is decorated with the crown of the Grand Duke and the inscription: "To His Imperial Highness, the Grand Duke Vladimir Aleksandrovich.” Perl. Jun. 18, 1931. Savitskii, S. Slavic Recon Project. Metropolitan New York Library Council, 2008. Shlissel'burg (Russia). Slav. Reserve (Photo) (Vidy goroda Shlisselburga) SLV 135 [Back cover]; Front cover: Ego Imperatorskomu Vysochestvu Velikomu Kniaziu Vladimiru Aleksandrovichu: Vidy goroda Shlissel'burga; [Shoreline in Shlissel'burg]; [A fortress in Shlissel'burg]; [Churches in Shlissel'burg]; [A pavilion in Shlissel'burg].

Vidy goroda Vytegry i eia okrestnostei. Ia. A. Pekarskii, 1884. 12 mounted Photographs, $33 \times 41 \mathrm{~cm}$. Title from original portfolio. Handwritten letterpress. Pekarskii, Ia. A. Church architecture - Russia-Vytegra. Vytegra (Russia). Slav. Reserve (Photo) (Vidy Goroda Vytegry) SLV 136 Vidy goroda Vytegry i eia okrestnostei/fotografiia Ia. A. Pekarskago 1884 goda. [Cover title]; Dom, v kotorom ostanavlivalis' ikh Imperatorskie Vysochestva Velikii kniaz' Vladimir Aleksandrovich i supruga Ego Mariia Pavlovna v gorode Vytegrie 1884 g.; Vytegra. Novaia tserkov' Srieteniia Gospodnia; Vytegra. Voskresenskii sobor; G. Vytegra. Voskresenskii prospekt i zdanie pravleniia putei soobshcheniia II-go okruga; G. Vytegra. Srietenskii sobor i lietnii Vytegorskii obshchestvennyi klub; Siversov pod'emnyi most $\mathrm{v}$ gorodie Vytegrie. prezhnei sistemy; Vytegra. Tserkov' na kladbishchie v imia vsiekh Sviatykh; Novaia chasovnia v 3-kh verstakh ot goroda Vytegry, na tom mieste, gde otdykhal Petr Velikii, vo vremia proiezda v g. Arkhangel'sk; Tserkov' Pokrova Presviatoi Bogoroditsy na Vytegorskom pogostie; Shliuz Sv. Natalii v miestechke Markovie bliz g. Vytegry po Mariinskoi sisteme; 
Mariinskii vodianoi sudokhodnyi put', dvie parallel'nykh linii novykh i starykh mnogokamernykh shliuzov: sv. Pavla, Vladimira i Fomy. (Na Deviatinskom pogostie.); Bereg Onezhskago ozera, u podoshvy mielovoi Andomskoi gory v 20 vers. ot g. Vytegry.

Vidy Kieva. Kiev, [18-?] 23 mounted postcards in portfolio, $12 \times 13$ cm. "Aug. 14, 1934." Slavic Recon Project. Metropolitan New York Library Council, 2008. Kiev (Ukraine)-Pictorial works. Slav. Reserve (Photo) (Vidy Kieva) SLV 137 Tserkov' Andreia Pervozvannago; Sofiiskii sobor; Vid Podola i naberezhnoi; Obshchi vid Kievo-Pecherskoi Lavry; Mikhailovskii monastyr'; Pamiatnik grafa Bobrinskago; Lavrskaia kolokol'nia i monastyrskii vkh[od]; Institut blagorodnykh dievits; Gorodskaia duma; Universitet Sv. Vladimira; Tsiepnoi most chrez Dniepr; Kreshchatikhskii pamiatnik; Bratskii monastyr'; Zhelieznaia tserkov'; Pamiatnik Bogdanu Khmel'nitskomu; Podol s terrasy Andreia Pervozvannago; Zolotyia vorota; Askol'dova mogila; Vid s vokzala na Kiev; Vid na staryi Kiev; Fasad Kievskago Dvortsa; Pamiatnik kniazia Vladimira; Vid Kreshchatika s sada mineral'nykh vod.

Vidy Kieva. Kiev: Perepletchik Strzhalkovskii, [190-?]. 24 mounted photographs in portfolio, $25 \times 18 \mathrm{~cm}$. Shcu, O. A. Kiev (Ukraine). Slav. Reserve (Photo) 99-5215 SLV 138 [Cover title]; Vid na Podol. Kiev; Sofiisk. Sobor v Kieve; Kiev. Pamiatn. Gr. Bobrinskomu; Kiev. Vid iz za Dniepra; Zhel. dor. v Kadetsk. roshchi Kiev; Kiev. Imper. Universitet sv. Vladimira; Novyi Dramaticheskii Teatr. Kiev; Mikhailovskii Monastyr. Kiev; Kiev. Zolotyia vorota; Alleia v Kupech. sadu. Kiev; Spasat. stants. na pristani. Kiev; Kiev. Vid na muzei; Tsarskii Dvorets v Kievie; Kiev. Pamiatn. Bogdanu Khmiel'nitskomu; Kiev. Politekhnicheskii institut; Pamiatn. Sv. Kn. Vladimiru. Kiev; Kiev. Bul'varnaia Ulitsa; Sobor sv. kn. Vladimira. Kiev; Pamiatnik Kreshcheniia v Kievie; Kiev. Gorodskaia Duma; Kiev. Vid iz za Dniepra; Tsiepnoi Most v Kievie; Monum. Imperatoru Nikolaiu I. Kiev; Vid na Tser. Andreia Pervozv. v Kievie.

Vidy Kishineva i ego okrestnostei. Kishinev, 1889?. 115 mounted photographs on 53 leaves $(2 \mathrm{v}$.), $31 \times 43 \mathrm{~cm}$. Binder's title: Kishinev i ego okrestnosti. "Fotogr. P. M. Kondratskago." "Bessarabskaia sel'sko khoziaistvennaia vystavka 1889 g.," v. $138-53$ (photos. 76-115). Photographs of panoramic view of the city folded on the first leaf. Slavic Recon Project. Metropolitan New York Library Council, 2008. Kondratskii, P. M. Chisinau (Moldova). Slav. Reserve (Photo) (Vidy Kishineva i ego okrestnostei) SLV 139 Vidy Kishineva i ego okrestnostei; Obshchii vid Kishineva s gory Ryshkanovki. (panorama); Obshchii vid Kishineva s gory Ryshkanovki. (panorama); Obshchii vid Kishineva s gory Ryshkanovki. (panorama); Obshchii vid Kishineva s gory Ryshkanovki. (panorama); Obshchii vid Kishineva s gory Ryshkanovki. (panorama); Granitsa sela 
Ryshkanovki. (map); Vokzal s linii; Vokzal s goroda; Nikolaevskaia ulitsa i Eparkhial'n. zhenskoe uchilishche; Ugol Gubernskoi i Aleksandrovskoi ulits; Aleksandrovskaia ulitsa. Doma mitropolii; Aleksandrovskaia ul. Mitropoliia i Konsistor; Vorota k soboru s Aleksandrovskoi ulitsy; Severnyia chast' goroda s sobornoi kolokol'ni; Aleksandrovskaia ul. Dukhovnaia seminariia i konsistoriia; Ugol Aleksandrovskoi i Seminarskoi ulits; Ugol Iasskoi i Aleksandrovskoi ul., kvartira Naslednika Tsesarevicha vo vremia kompanii 76 goda; Aleksandrovskaia ulitsa. Pamiatnik Imperatoru Aleksandru II; Otkrytie pamiatnika Imperatoru Aleksandru II; Pamiatnik Pushkinu v gorodskom sadu; Aleksandrovskaia ulitsa. Kvartira Gosudaria Imperatora v 76 g.; Aleksandrovskaia ulitsa. Liuteranskaia tserkov'; Aleksandrovskaia ul. $\mathrm{k}$ vostoku s kolokol'ni Liuteranskoi tserkvi; Aleksandrovskaia ul. k zapadu s pozharnoi kolanchi; Iuzhnaia chast' goroda s pozharni kolanchi; Aleksandrovskaia ul. Tsyrk i Okruzhnyi sud; Gostinaia ulitsa; Gostinaia ulitsa. Kostel'; Nikolaevskaia ul. Kvartira Glavnokomanduiushchago v 76 g.; Ugol Galdinskoi i Fontannago pereulka. Okruzhnyi sud; Priemnaia Okruzhnago suda; Kievskaia ul. Pervaia muzhskaia klas. Gimnaziia; Ugol Podol'skoi i Gubernskoi ul., Zhenskaia Zemskaia gimnaziia; Ugol Gubernskoi i Reniskoi ulits. Real'noe uchilishche i dukhovn. muzhskoe; Ugol Gubernskoi i Reniskoi ulits. Real'noe uchilishche; Ugol Reniskoi i Boiukanckoi ulits; Pnevmaticheskyia lechebnitsa; Sennyia ploshchad'. Tieremnyi zamok; Sadovaia ulitsa; Sadovaia ulitsa; Min'kovskaia ulitsa; K[statenolomnaia?] ulitsa i Inzova gora; Severnaia chast' goroda. Staryi bazar; Fontan; Fontan; Vodovoz; Parovaia mel'nitsa; Tolkuchii rynok. Sapozhniki; Tolkuchii rynok. Tochil'shchik; Ulichnyi sapozhnik; Prodavets chesnoka; Evrei ravnoshchik bublikov. Vol. 2: Ulichnyi prodavets bragi [prokhladitel'nyi napitok]; Tochil'shchik zimoi; Tochil'shchik letom; Tsygan-kuznets; Tip kishinevskago evreia; Peizazh na r. Byk; Vodianaia mel'nitsa na r. Byk; Tenberskaia zastava; Ryshkanovka i predmest'e Kishineva; Kolodets na Tenberskoi zastave; Strel'ba na Ryshkanovke. (four images); Tsyganskii shater; Tsygane; Proizvodstvo kirpicha; Gonchar; Zhenshchina-goncharitsa; Ovtsevodstvo; Taban; Taban zimoiu; Taban lietom; Moldavanin; Izby moldavan; Vinodelie/Kashirnoe vino/; Vinodelie; Oroshenie ogorodov; Glavnyi vkhod na sel'sko-khoziaistv. Vystavku; [Plate reads: Bessarabskaia Sel'sko khoziaistvennaia vystavka 1889]; Leovskaia ulitsa/Zdanie sel'sko-khoziaistv vystavki/; Moment ob'iavleniia nagrad; Obshchii vid pavil'enov; Pavil'en kniazia Iu. Gagarina i Ko; Kamennyi otdel; Pavil'on g. Donicha; Vetriannyia vodokachka; Pavil'on g. Kristi; Pavil'on kniazia Manukbeia; Pavil'on - konditorskaia; Otdel domashnyi ptitsy; Mashinnyi otdel S. Serbova; Mashinnyi otdel g. Ekkerty. [Plate reads: Adolf Veistman i Ko., Odessa]; Mashinnyi pavil'on K. Gartinga; Mashinnyi otdel D. Ransom; Kustarnyi otdel/Tarakliiskoe remelennoe uchilishche/; Kustarnyi otdel; Vinnyi otdel g. Semigradova; Vinnyi otdel g. Tomul'tsa; Marko D[ykii]/Deputat 
s. Dolinian Khorinsk. u./; Ivan Kampri[u]/Deputat s. Dolinian Khorinsk. uezda/; Rumynskii orkestr; Zavodov g.g. Suruchana i Russo. (two images); Zavoda g.g. Buzni i Russo. (two images); Zavoda g. Syrychana. (two images); Zavoda g. Syrychana. (two images); Zavoda g. Feodos'eva. (two images); Zavoda g. Buzni. (two images); Zavoda g. Semigradova. (two images); Zavoda g. Brodskago. (two images); Zavoda g. Semigradova. (two images).

Vidy Krasnago Sela. Fotografia N. Digo. 23 mounted photo$=\pi-$ Trim graphs, 30 x $40 \mathrm{~cm}$. Markings: 10/21/33 (plate 1, in pencil), "Perl. Jun 18, 1931." Cover title. N. Digo Fotogr. Gosudarstvennago Konnozavodstva. Slavic Recon Project. Metropolitan New York Library Council, 2008. Horses - Breeding Russia. Krasnoselskoe (Leningradskaia oblast, Russia). Slav. Reserve (Photo) (Vidy Krasnago sela) SLV 140 [Cover title]; [Houses in Krasnoe Selo]; [Gauptvakhta]; [Voennyi gospital]; [Shtabnyi dom avangardnago lageria]; [Voennyi gospital]; [A modern house with a shoe store and firemen]; [Krasnoe Selo]; [Krasnosel'skaia tserkov']; [General view of Krasnoe Selo]; [A building near river]; [Stantsiia Krasnoe Selo]; [Tsarskaia stolovaia]; [Dvorets E.I.V. Velikago Kniazia Mikhaila Nikolaevicha]; [An imperial palace]; [Houses]; [A double tent on the field].

Vidy Peterburga. St. Petersburg: P. I. Babkin, 1895. 71 mounted photographs, $24 \times 34 \mathrm{~cm}$. Purchased from Alex Rabinovich. Cover by Konst. Izenberg. Captions in French, German and Russian. Babkin, P. I. Izenburg, Konst. Saint Petersburg (Russia). Slav. Reserve (Photo) 99-1505 SLV 141 [Cover title]; [Title page]; Pamiatnik Petru Velikomu. raboty Fal'koneta (Senatskaia ploshchad'); Domik Petra Velikago i v nem chasovnia Spasitelia (Peterburgskaia storona); Troitskii sobor, osnov. v 1703 g. (Peterburgskaia storona); Dom Petra Velikago (v Lietnem sadu); Aleksandro-Nevskaia Lavra (Naruzhnyi vid); Petropavlovskaia kriepost'; Sobor sv. Troitsy, osnov. v 1703 g. (Peterburgskaia storona); Anichkin [Anichkov] Dvorets; Vid Nevskago prospekta (Ot Politseiskago mosta k Admiralteistvu); Imperatorskii Zimnii Dvorets; Zimniaia Kanavka; Zimnii Dvorets i Admiralteistvo (Vid s Vasil'evskago Ostrova); Pamiatnik Ekaterinie Velikoi (raboty M. O. Mikieshina); Smol'nyi monastyr' (naruzhnyi vid); Kazanskii Sobor; Inzhenernyi Zamok; Aleksandrovskaia kolonna (Ploshchad' Zimniago Dvortsa); Dvortsovaia ploshchad'; Pamiatnik Imperatoru Nikolaiu I (Nikolaevskaia ploshchad'); Isakievskii sobor; Vid ot Siniago mosta; Arka Glavnago Shtaba; Nikolaevskii Most (Vid s Vasil'evskago Ostrova); Imperatorskii Ermitazh; Akademiia Khudozhestv; Preobrazhenskii vsei gvardii Sobor; Anichkin most; Anichkin most (fig. 1-ia) rabota Barona Klodta; Anichkin most (fig. 2-ia) rabota Barona Klodta; Anichkin most (fig. 3-ia) rabota Barona Klodta; Anichkin most (fig. 4-ia) rabota Barona Klodta; Vid Nevskago prospekta (ot Anichkina Dvortsa k Admiralteistvu); Birzha; Tamozhnia; Birzha (vid s Dvortsovago mosta); Vid Nevskago prospekta 
(ot Publichnoi biblioteki k Admiralteistvu); Vid Nevskago prospekta vo vremia lietniago remonta (ot Gorodskoi Dumy k Nikolaevskomu vokzalu); Vokzal Nikolaevskoi zhel. dor.; Naberezhnaia Vasil'evskago ostrova (16 i 17 linii); Fontanka u Anichkina mosta; Naberezhnaia Vasil'evskago ostrova (vid s Nikolaevskago mosta); Aleksandrinskii teatr; Moika; Siennoi rynok; Chasovnia u Lietniago sada; Novodievichii Monastyr'; Pamiatnik Slavy; Sobor sv. Troitsy i pamiatnik Slavy (Izmailovskii polk); Fontanka (vid s Anichkina mosta); Imperatorskoe Uchilishche Pravoviedeniia; Vokzal S.-PeterburgskoVarshavskoi zhel. dor.; Mikhailovskaia artilleriiskaia Akademiia i Uchilishche (Vyborgskaia storona); Mariinskii teatr; Tsirk Chinizelli; Troitskii most (vid na Lietnii sad); Morskoi kanal; Morskoi kanal i Port; Morskoi port; Pamiatnik Ekaterinie Velikoi (Nevskii, Ekaterininskii skver); Mikhailovskaia ulitsa (vid s Nevskago prosp.); Gorodskaia Duma; Nevskii prospekt u Gostinago Dvora; Pristan' na Nevie (Vasil'evskii ostrov); Vid Nevskago prospekta (ot Gostinago Dvora k Admiralteistvu); Nevskii u Miliutina riada; Admiralteiskaia naberezhnaia (vid cherez Dvortsovyi most); Novoe Admiralteistvo (vid chasti Angliiskoi naberezhnoi); Bol'shaia Morskaia (vid ot Reformatskoi kirkhi); Vid na Sredniuiu Nevku; Dvortsovaia ploshchad' (vo vremia torzhestvennago bogosluzheniia).

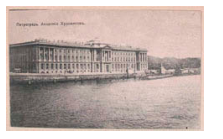

[Vidy S.-Peterburga]. [Petrograd: Akademiia khudozhestv, [191-?] 20 mounted postcards, $25 \times 31 \mathrm{~cm}$. Title from the shelflist card. Captions in French and Russian. 20 postcards (Carte Postale) picturing St. Petersburg (Petrograd) before the October revolution. Radetskii, P.S. Saint Petersburg (Russia)- Pictorial works. Slav. Reserve (Photo) (Vidy S. Peterburga) SLV 142 Petrograd. Akademiia Khudozhestv; S.-Peterburg. Imperatorskaia Akademiia Nauk. Zoologicheskii muzei; Petrograd. Aleksandrovskii sad. Pamiatnik Przheval'skomu; Petrograd. Anichkin dvorets; St. Peterburg. Botanicheskii sad Kal'marius; Petrograd. Domik Petra I v Lietnem Sadu; Petrograd. Mariinskii dvorets; Petrograd. Mariinskii teatr; Petrograd. Moskovskiia Vorota; S. Peterburg. Mramornyi Dvorets; Petrograd. Neva i Naberezhnaia. Vas. Ostrov; Petrograd. Nikolaevskaia Akademiia General'nago Shtaba; Petrograd. Pamiatnik Petra Velikago; Petrograd. Pamiatnik "Steregushchemu"; S. Peterburg. Petropavlovskaia Liuteranskaia tserkov'; S. Peterburg. Petrovskiia vorota; Petrograd. Pochtamp [plate stamped "Leningrad"]; S. Peterburg. Risoval'naia shkola Barona Shtiglitsa; Petrograd. Tavricheskii dvorets. (Gosudarstvennaia Duma); St. Peterburg. Elagin ostrov. Strielka.

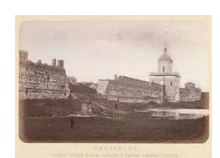

Vidy Smolenska i Borodinskago polia. Moscow: Fotograf M. Nastiukov, [18-?] 14 mounted photographs, $40 \times 53 \mathrm{~cm}$. Binder's title. Album of photographs with descriptive letterpress, originally in box. Stamp on cover reads "Fotograf ego Imperatorskago Vysochestva Gosudaria Naslednika Tsesarevicha M. Nastiukov v Moskvie u Mias. Vorot.” Nastiukov, M. Slavic Recon Project. Metropolitan New York Library Council, 2008. Smolensk 
(Russia). Borodino (Mozhaiskii raion, Russia). Slav. Reserve (Photo) (Vidy Smolenska i Borodinskago polia) SLV 143 [Cover title]; Vid goroda Smolenska; Vid goroda Smolenska; Smolensk. Obshchii vid ot bashni Veselukhi; Smolensk. Uspenskii sobor; Smolensk. Kriepost' postroena Borisom Godunovym i pamiatnik polkovniku Engel'gardu ubitomu pod Smolenskom v 1812 godu; Smolensk. Pamiatnik 1812 goda; Kolotskii Monastyr' osnovan v 1413 godu blagoviernym kniazem Andreem Dmitrievichem. Bliz st: Uvarovskoi; Moskovsko-Smolenskoi zheleznoi dorogi; Kolotskii Monastyr' osnovan v 1413 godu blagoviernym kniazem Andreem Dmitrievichem. Bliz st: Uvarovskoi Moskovsko-Smolenskoi zheleznoi dorogi; Borodinskoe pole na kotorom bylo general'noe srazhenie russkikh s frantsuzami v 1812 g.; Selo Borodino. Dvorets sobstvennost' Ego Imperatorskago Velichestva Gosudaria Imperatora; Selo Borodino. Dvorets sobstvennost' Ego Imperatorskago Velichestva Gosudaria Imperatora; Borodinskoe pole. Monument (v pamiat' Borodinskoi bitvy v 1812 godu); Borodinskoe pole. Borodinskii Spasskii Monastyr' osnovan, suprugoiu ubitago v Borodinskoi bitvie Generala Tuchkova 25 avgusta 1812 goda; Borodinskoe pole. Borodinskii spasskii monastyr' osnovan, suprugoiu ubitago v Borodinskoi bitvie Generala Tuchkova 25 avgusta 1812 goda.

Vidy Sviato-Troitskoi Sergievoi Lavry i eia okrestnostei. 40 mounted photographs, $31 \times 45 \mathrm{~cm}$. Binder's title. On cover: "PEREPL. vys. utv. t-va I. N. Kushnerev i Ko. v Moskvie" Slavic Recon Project. Metropolitan New York Library Council, 2008. Troitse-Sergieva lavra (Russia). Icons, Russian. Church buildings - Russia. Slav. Reserve (Photo) (Vidy Sviato-Troitskoi Sergievoi Lavry i eia okrestnostei) SLV 144 [Cover title]; Khram v chest' Chudotvornoi ikony Chernigovskoi Bogomateri; Obshchii vid peshcher pri Gefsimanskom Skitie; Kladbishchenskaia tserkov' Gefsimanskago Skita; Trapeznaia tserkov' Gefsimanskago Skita; Vnutrennost' dereviannoi tserkvi Gefsimanskago Skita; Dereviannaia drevniaia tserkov' Gefsimanskago Skita; Kladiaz; Vid posada, s vostochnoi storony, s Lavrskoi kolokol'ni; Vid posada s zapadnoi storony; Sv. vrata Lavry; Kolokol'nia; Vid posada s sievernoi storony; Tserkov' Pr. Mikheia; Moskovskaia Dukhovnaia Akademiia; Tserkov' Ioanna Predtechi nad sv. vratami; Vnutrennost' tserkvi Smolenskoi ikony Bogomateri; Tserkvi: Smolenskoi ikony Bogomateri i Bol'nichnaia vo imia svv. Zosimy i Savvatiia; Uspenskii Sobor; Orel v pamiat' spaseniia Petra I-go v Uspenskom Soborie; Vnutrennii vid Uspenskago Sobora; Trapeznaia tserkov' vo imia Prepodobnago Sergiia; Vnutrennost' Trapeznoi tserkvi; Tserkov' Soshestviia Sv. Dukha; Tserkov' Prepodobnago Nikona; Obshchii vid Lavry s iugo-vostochnoi storony; Troitskii Sobor; Izobrazhenie raki, v kotoroi pokoiatsia sv. moshchi Pr. Sergiia; Prikhodskaia tserkov' Proroka Ilii v Sergievskom posadie; Prikhodskaia tserkov' sv. Apostolov Petra i Pavla v Sergievskom posadie; Prikhodskaia tserkov' Proroka Ilii 
(dereviannaia); Prikhodskaia tserkov' Rozhdestva Khristova v Sergievskom posadie; Prikhodskaia tserkov' Vozneseniia Khristova v Sergievskom posadie; Prikhodskaia tserkov' sv. Nikolaia v Sergievskom posadie; Vid Zosimovoi Pustyni; Vnutrennost' tserkvi v Pustyni sv. Paraklita; Obshchii vid Pustyni sv. Paraklita; Vid Vifanskoi Dukhovnoi Seminarii; Vnutrennost' Vifanskoi tserkvi: gora Favor; Obshchii vid Vifanii; Obshchii vid Kinovii.

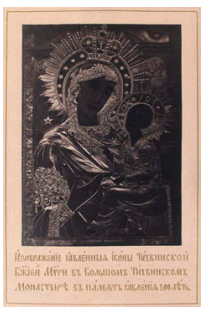

Vidy Tikhvinskago bol'sh. monastyria. V pamiat' piatisot [sic] lietiia. 1383-1883. 1883. 10 mounted photographs, $21 \mathrm{x}$ $31 \mathrm{~cm}$. "10 albumen prints, originally bound in hardcover album with gilt titles, now separately preserved with cover in archival box. Prints of various sizes, oval: 6 1/4 x 11 1/8"; rectangular: $67 / 8 \times 87 / 8$ "; titled on mounts in gold ink in calligraphic Slavonic script. 2 general views from south and east. 5 views of various churches: Dormition, in which the Icon of the Virgin of Tikhvin is kept; Protection and Belltower; 'Krylechko' with the miraculous icon painted on the wall; Erection of the cross; Ascension; 1 general view of town; 2 images of an icon; Altar with the Icon of the Virgin of Tikhvin; and a representation of the Icon in the main monastery of Tikhvin, commemorating 500 years since the apparition. Excellent group of documentary images. The general views are particularly good and the view of the city is outstanding." (Pierre Apraxine). DOE Title II-C "Russian Illustrated Books and Photographs" Project. ${ }^{* Z Q-1004 ~ \& ~}$ Master Negative: ${ }^{\star}$ ZZ-30647 Monasteries-Russia (Federation)-TikhvinTikhvinskaia Bogomater (Icon). Church architecture-Russia- Tikhvin. Slav Reserve (Photo) (Vidy Tikhvinskago) SLV 145 [Binder's title]; Izobrazhenie iavlenniia ikony Tikhvinskoi Bozhiei Materi $\mathrm{v}$ Bol'shom Tikhvinskom Monastyrie v pamiat' iavleniia 500 liet; Vid Tikhvinskago Bol'shago Monastyria s iuzhnoi storony; Vid Tikhvinskago Bol'shago Monastyria s vostochnoi storony; Uspenskii sobor Tikhvinskago Bol'shago Monastyria, gdie samaia iavlennaia ikona Tikhvinskoi Bozhiei Materi; Pokrovskaia tserkov' i kolokol'nia v Tikhvinskom Bol'shom Monastyrie; Tikhvinskaia tserkov' (Krylechko) gdia chudotvornaia ikona Prestyia B.d.ts. $\mathrm{y}$, pisannaia na stienie; Kresto-Vozdvizhenskaia tserkov' v Tikhvinskom Bol'shom Monastyrie; Voznesenskaia tserkov' v Tikhvinskom Bol'shom Monastyrie; Izobrazhenie sieni i iavlennoi Ikony Tikhvinskoi Bozhiei Materi v Uspenskom soborie Tikhvinskago Bol'shago Monastyria; Vid goroda Tikhvina.

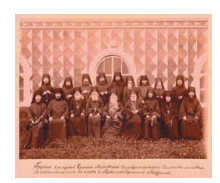

Vidy tserkvei i zdanii Moskovskago stavropigial'nago pervoklassnago Simonova monastyria: 28-go marta 1893 goda. 16 mounted photos, $33 \times 41 \mathrm{~cm}$. Marked "Bolan 9 Jan. 34." Stamp on verso of plate 16 "MUZEI GORODA 10280." Binder's title. "Fotogr. P. Ostroumov." Binding of purple plush, gilt. Ostroumov, P. Slavic Recon Project. Metropolitan New York 
Library Council, 2008. Moskovskii Simonov monastyr'. Church buildings Russia. Monasteries, Orthodox Eastern -Russia (Federation) -Moscow. Slav. Reserve (Photo) (Vidy tserkvei i zdanii Moskovskago stavropigialnago pervoklassnago Simonova monastyria) SLV 146 [Cover title]; Obshchii vid Moskovskago stavropigial'nago Simonova monastyia, (s sievernoi storony); Vid Simonova monastyria. (s zapadnoi storony); Prud, iskopannyi prepod. Sergiem, s pervymi inokami obiteli sei. (s iuzhnoi storony); Vid restavriruimago Uspenskago khrama Simonovoi obiteli, sooruzhennago v 1379-1405 g. g. (s zapadnoi storony); Vid Uspenskago sobora, Simonovoi obiteli, (s iuzhnoi storony); Vid Tikhvinskoi trapeznoi tserkvi, v Simonovom monastyrie; Vid drevnei Spasskoi tserkvi, nad zapadnymi vratami, (s vostochnoi storony); Vid Spasskoi tserkvi proiskhozhdeniia chestnykh drev, i zapadnykh sviatykh vrat snaruzhi; Tserkov' sv. Nikolaia chudotvortsa. (s zapadnoi storony). S bratskimi kelliiami [last part of caption is handwritten]; Tserkov' prepod. Aleksandra Svirskago. (s zapadnoi storony); Vid Simonovskoi kolokol'ni i, bratskikh kellii. (s seviero-zapadnoi storony); Vid dvortsa, sooruzhennago tsarem Feodorom Aleksieevichem, i bashni dulo imenuemoi sluzhivshei boinitseiu-pri osadakh (s seviernoi storony); Sad Simonova monastyria s prilegaiushchimi k nemu stroeniiami. vnutri dvora [last three words handwritten]; Vid tserkvi Rozhdestva Bogoroditsy, na starom Simonovie, osnovannoi prepod. Feodorom v 1370 godu. (s iuzhnoi storony); Grobnitsy, Peresvieta i Osliabia, doblestnykh inokov Sergievykh, pochivaiushchikh na Starom Simonovie; Gruppa starshei bratii Moskovskago Stavropigial'nago Simonova monastyria, s nastoiatelem vo glaviu o arkhimandritom Andreem [whole of caption is handwritten]; Vid Moskvy ot Simonovia monastyria Gruppa starshei bratii Simonova monastyria s nastoiatelem vo glave o. arkhimandritom Andreem [back of item].

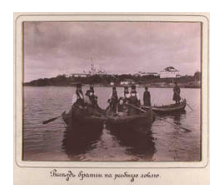

Vidy Valaamskago monastyria. [S.l., s.n., ca. 1890]. 40 photographs on 21 leaves, $26 \times 37 \mathrm{~cm}$. Master Negative: ${ }^{\star} Z Z-30646 /$ ${ }^{\star}$ ZQ-1009 Bookplate of Grand Duke Vladimir Aleksandrovich. Pencil note on final page: "K.P. No. 42, 14 Ian. 1904 g." "Leather bound album, all edges gilt, of 40 bromide plates of identical size (6 9/16 x $87 / 8$ ") mounted back to back into the page (as in carte de visite albums). Titled on the cover binding: 'Views of the Monastery of Valaam.' Written in pencil on the second: Hammer, May 3, 1945. Album documents the Monastery of Valaam, various hermitages (All Saints, St. Nicholas, etc.), natural settings, and occupations of the monks. Includes a general view of the Monastery, with processions or interior views of churches, tombs, grottos, and retreats of holy monks. Then follows diverse activities: construction of church, fishing in the lake, harvesting cabbages or hay. Follows nature views of cliff, and shores of Lake Valaam, holy monk's abandoned huts, etc., generally with monks posing in foreground. All photos are in Russian in calligraphic script. Album depicts in post-card images the life, architecture, 
and settings of the monastery. As such it is a very general but good introduction. None of the photographs are signed. The date is suggested by the bromide process." (Pierre Apraxine). DOE Title II-C "Russian Illustrated Books and Photographs" Project. Valaamskii monastyr. Monasteries, Orthodox Eastern-Russia (Federation)-Valaam Island. Slav Reserve (Photo) (Vidy Valaamskago) SLV 147 [Cover title]; Pervoklasnyi, Spasopreobrazhenskii, Valaamskii Monastyr'; $\mathrm{Na}$ rybnoi lovlie; Prazdnichnyi Vykhod bratii iz tserkvi v trapezu s Prechistoiu; Vykhod O. Igumena v tserkov' na prazdnik Preobrazheniia; Skit Vsekh Sviatykh; Vstrecha krestnago khoda na prazdnik Vsekh Sviatykh; Skit Sviat. Nikolaia Chudotvortsa; Vnutrennii vid tserkvi sviat. Nikolaia; Skit sv. Ioanna Predtechi; Vnutrennii vid tserkvi Proroka Predtschi; Skit prep. Aleksandra Svirskago; Vnutrennii vid tserkvi prep. Aleksandra Svir.; Skit sv. Proroka Ilii; Vnutrennii vid tserkvi sv. Proroka Ilii; Skit prep. Avramiia Rostovskago; Vnutrennii vid tserkvi prep. Avramiia Rostovskago; Konevskii skit; Vnutrennii vid tserkvi vo imia Konevskiia B.Materi; Kladbishchenskaia tserkov' na Valaamie; Vnutrennii vid Kladbishchenskoi tserkvi; Mogila O. Igumena Damaskina; Pechera prep. Aleksandra Svirskago; Pustyn'ka skhimonakha Nikolaia; U mogily skhimonakha Nikolaia; Na postroikie sobora; Vyezd bratii na rybnuiu lovliu; Bratiia na rubkie kapusty; Bratiia na senokose; Kukinskiia skala na Valaamie; Valaamskiia skaly; Iz mestnostei O. Valaama; Lesnaia glush zimoiu; Bereg O. Valaama; Bereg O. Valaama; Iz mestnostei O. Valaama; Pustynka skhimonakha Antoniia; Kukinskiia skaly na Valaamie; Iz vidov O. Valaama; Skaly Il'niskago skita; Valaamskie rybaki.

Vidy Vserossiiskoi khudozhestvennoi promyshlennoi vystavki 1896 g. Nizhnem Novgorodie. Moscow: Fototipiia Sherer, 1896? 200 mounted photographs, $33 \times 43 \mathrm{~cm}$. No text. Two hundred plates on 100 leaves, with descriptive letterpress. With metal monogram of Grand Duke Konstantin Konstantinovich and crown on binding (crown missing). Industries - Russia - Nizhnii Novgorod Exhibitions. Exhibitions - Russia - Nizhnii Novgorod. Slav. Reserve Photo (Vidy vserossiiskoi khudozhestvennoi promyshlennoi vystavki 1896 g. Nizhnem Novgorodie) SLV 148 [Binder's title]; Glavnyi Vkhod; Glavnyi Vkhod; Obshchii vid (ot glavnago vkhoda); Obshchii vid (peredniaia chast' sada - vid na obelisk zdaniia Administratsii i Gostinnitsy); Obshchii vid; Vid na bassein i zdanie Sredne-Aziatskago otdela; Obshchii vid; Obshchii vid; Obshchii vid (pavil'ony: Tsarskii, Meteorologii, Kitaiskii i dr.); Imperatorskii pavil'on; Imperatorskii pavil'on; Obshchii vid (Tsarskii pavil'on i prilegaiushchaia $\mathrm{k}$ nemu chast' sada); Obshchii vid (Tsarskii pavil'on, zdanie voennago i voenno-morskago otdela; sad glavnago upravleniia udelov); Khudozhestvennyi otdel; Zdanie khudozhestvennago otdela; Khudozhestvennyi otdel; Fasad khudozhestvennago otdela; Obshchii vid; Pavil'on glavnago upravleniia udelov; Obshchii vid; Pavil'on glavnago upravleniia departamenta udelov; Pavil'on "Krainii Sever"; Obshchii vid (pavil'on K.G. 
Makovskago; Khudozhestvennyi otdel); Panorama - "Pokorenie Kavkaza," Kartina Rybo; Teatr i kontsertnyi zal; Ikh Velichestva prisutstvuiut pri svobodnom polete shara uchebnago vozdukhoplavatel'nago parka; Uchebnyi vozdukhoplavatel'nyi parka; Voenno-morskoi otdel; Morskoi otdel - Vodolaznoe delo; Pavil'on Kn. L.S. Golitsyna; Pavilion "Krasnyi krest"; Nachal'noe sel'skoe uchilishche M.N. P.; Nauchno-uchebnyi otdel (XIX); Obraztsovoe zdanie tserkvi-shkoly; Blagotvoritel'nyia uchrezhdeniia; Pavil'on vedomstva uchrezhdenii imperatritsy Marii i drugikh blagotvoritel'nykh uchrezhdenii; Voskresnyia shkoly; Obshchii vid (peredniaia chast' sada- vid na obelisk, vkhodnuiu kolonnadu); Elektricheskaia doroga; Obshchii vid; Obshchii vid tsentral'nago zdaniia; Glavnyi vkhod v manufakturnyi otdel; Pavil'on dlia orkestra v tsentral'nom sadu; Otkrytie vystavki (podniatie flagov); Obshchii vid; Kitaiskii pavil'on; Kitaiskii pavil'on; Pavil'on D.N. Lebedeva; Kompaniia Krengol'mskoi manufaktury; Pavil'on S. A. Prokof eva i Ko.; Pavil'ony T-va Abrikosova synovei i Br. Popovykh; Pavil'on Br. Eliseevykh; Fabrichno-zavodskii otdel (IX); Sadovodstvo, Plodovodstvo i Ogorodnichestvo; Pavil'on E. Immer i Syn.; Zdanie ptitsevodstva; Obshchii vid; Aziatskii otdel; Sredne-Aziatskii otdel; Zdanie administratsii vystavki; Zdanie administratsii vystavki; Mashinnyi otdel.; Mashinnyi otdel.; Vkhod v glavnoe zdanie machinnago otdela; Obshchii vid; Stantsiia zheleznoi dorogi; Vodonapornaia bashnia inzhenera A. V. Bari; Mashinnyi otdel (Dobavochnoe zdanie); Obshchii vid; Sibirskii otdel (XIII); Pavil'on O-va Brianskago zavoda; Olonetskii Bassein; Pavil'on Bogoslovskago gornago okruga; Obshchii vid (chastnye pavil'ony gornago otdela); Pavil'on Dneprovskago metallurgicheskago O-va.; Pavil'on Nizhnetagilskikh gornykh zavodov (Naslednikov P. P. Demidova); Pavil'on Votkinskago kazennago zavoda; Pavil'on O-va Mal'tsovskikh zavodov; Obshchii vid (pavil'on Mal'tsovykh, Nizhne-tagil'skikh zavodov i dr.); Obshchii vid; Pavil'on Amurskoi zolotopromyshlennosti; Pavil'on O-va dlia proizvodstva tsementa i drugikh stroitel'nykh materialov; Obshchii vid; T-vo Artezianskago vodosnabzheniia B. I. Fon-Vangel; Pavil'on Sibirskoi zheleznoi dorogi; Obshchii vid (eksponaty Rudzskago, Bergengeima); Obshchii vid; Pavil'on T-va S. M. Shibaev i Ko.; Pavil'on KaspiiskoChernomorskago neftepromyshlennago O-va.; Obshchii vid; Pavil'on "Sormovo" O-va staleliteinykh zavodov; Pavil'on O-va Riazansko-Ural'sko zhe.dor.; Otdel zheleznykh dorog (stantsiia elektricheskoi signalizatsii); Otdel XVII pavil'on zheleznykh dorog; Obshchii vid (palatki kebke, neftianyia bashni i dr.); O-vo spasaniia na vodakh; Pavil'on "Rechnago torgovago sudokhodstva"; Volzhsko-Kaspiiskoe sudokhodstvo; Zdanie kustarnago otdela; Zdanie ekipazhnago otdela (IX); Pavil'on T-va Br. Nobel; Obshchii vid (kolokol'nyi riad, Nobel i drug.); Obshchii vid; Kolokol'nyi riad (Pavil'on Finliandskago, Olovianishchnikova i drug.); Pavil'on goroda Moskvy; Pavil'on G.G. Adel'khanova; Obshchii vid; Obshchii vid (Mel'nitsy Davydova, Finliandskii pavil'on i dr.); Obshchii vid; Sel'skokhoziaistvennyi otdel.; Sel'sko-khoziaistvennyi otdel.; Sel'sko-khoziaistvennyi otdel (Dobavochnyi); Zdanie pozharnago oboza; Lesovodstvo; Pavil'on S. P. FonDerviz; Obshchii vid; Ogneupornyia postroiki; Dom narodnykh razvlechenii 
i besed; Obshchii vid; Vid na N.-Novgorod ot sibirskoi pristani; Khudozhestvennyi otdel (skul'ptura); Khudozhestvennyi otdel; Vedomstvo uchrezhdenii imperatritsy Marii; Vedomstvo uchrezhdenii imperatritsy Marii; Sibirskii otdel; Ekaterinburgskaia granil'naia fabrika; Sredne-Aziatskii otdel; Sredne-Aziatskii otdel; Promysly krainiago severa; Khudozhestvennopromyshlennyi otdel (X); Khudozhestvenno-promyshlennyi otdel (X); Khudozhestvenno-promyshlennyi otdel (X); Stroganovskoe uchilishche tekhnicheskago risovaniia (X); Grot v pavil'on Kn. L. S. Golitsyna; Otdel VIII; Manufakturnyi otdel.; Manufakturnyi otdel.; Manufakturnyi otdel.; Manufakturnyi otdel.; Manufakturnyi otdel.; T-vo Tverskoi Manufaktury; T-vo Prokhorovskoi Trekhgornoi Manufaktury; T-vo Nikol'skoi Manufaktury Savvy Morozova syn i Ko.; T-vo Zhirardovskikh manufaktur; T-vo Tiulevoi fabriki; Obraztsy pervobytnykh Ivanovskikh sittsev s 1691 po 1850 g. (Iz sobraniia D.G. Burylina); Obshchii vid Gornozavodskago otdela; Gornozavodskii otdel.; Gornozavodskii otdel.; Gornyi otdel (VII); Gornozavodskii otdel.; Gornozavodskii otdel.; Obshchii vid Gorno-zavodskago otdela; Gornozavodskii otdel (VII); Gornozavodskii otdel (VII); Goroblagodatskii gornyi okrug; Gornozavodskii otdel.; Ekaterinskii zavod (gornyi otdel VII); Sysertskie zavody (gornyi otdel VII); Kulebakskii gornyi zavod; Kazhimskie zavody (gornyi otdel VII); T-vo Serginsko-Ufaleiskikh zavodov (gornyi otdel VII); O-vo Putilovskikh zavodov; Simskie zavody (gornyi otdel VII); O-vo Beloretskikh zhelezodelatel'nykh zavodov Pashkovykh (gornyi otdel VII); Kyshtymskie Gornye Zavody; Provolochnyi zavod Beker i Ko. v Libave; Pavil'on Donetskikh uglei Solepromyshlennikov; Voenno-morskoi otdel; Obkhovskie staleliteinye zavody; Kronshtadskii parokhodnyi zavod; Mashinnyi otdel (XII); Mashinnyi otdel.; Mashinnyi otdel (XII); Mashinnyi otdel.; Mashinnyi otdel.; Mashinnyi otdel.; Mashinnyi otdel.; Mashinnyi otdel.; Mashinnyi otdel (Dobavochnyi); T-vo Moskovskago Metallicheskago zavoda; Pozharnyia truby Gustava List.; Obshchii vid IX otdela; Obshchii vid IX otdela; Severnoe stekol'noe obshchestvo; T-vo Einem; Torgovyi dom A.M. Zhukova; Obshchii vid IX otdela; Proizvodstva Fabrichno-zavodskiia; Obshchii vid IX otdela; Obshchii vid IX otdela; Fabrichnozavodskii otdel (IX dobavochnyi); Proizvodstva Fabrichno-remeslennyia (IX); Proizvodstva Fabrichno-remeslennyia (IX); Kustarnye promysly; Kustarnye promysly; Kustarnye promysly; Lesnoi otdel (VI); Lesnoi otdel (torfianoe proizvodstvo).

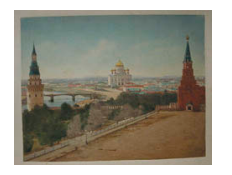

[Views of Saint Petersburg and Moscow]. [S.l: s.n, 1880?] 21 photographs (2 col.), $32 \times 40 \mathrm{~cm}$. Unbound, loose photographs, mounted on loose cards. Albumen prints, two handcolored. Handwritten captions in Russian, French and/or English. Saint Petersburg (Russia). Moscow (Russia). Photography-Russia19th century. Slav. Reserve (Photo) 07-6537 SLV 153

1. Kremlin Palace - St. George Ritter Saal; 2. Kremlin Palace - Thron Saal/ Granite Hall (Granovitaia Palata); 3. View on Moscow and river from 
Kremlin; 4. General View of city from the Kremlin; 5. View on river Neva; 6. Winter Palace; 7. Winter Palace (Marine building); 8. Triumphal chariot on the arch of the Main Staff; 9. Moskau - Majlania Dvor (the prison); 10. Church of Christ the Saviour; 11. Church of Christ the Saviour: ceiling painting; 12. Church of Christ the Saviour; 13. The Hermitage; 14. Admiralty Palace; 15 . The monument to the Catherine the Great; 16. St. Isaac's Cathedral; 17. Nevsky Prospect and view on Admiralteistvo; 18. Winter Palace and Alexandrovskaia Colonna; 19. Monument to Peter the Great from Catherine the Second; 20. Kazan Cathedral; 21. View on Petropavlovsk Fortress.

Vsemirnaia Kolumbova vystavka 1893 goda $v$ Chikago: Russkii otdiel. [St. Petersburg: A. Petersena, 1893]. 20 mounted photographs on 10 leaves, $35 \times 46 \mathrm{~cm}$. Captions in manuscript. Monogram of Grand Duke Vladimir Aleksandrovich affixed to cover. With stamp of: "Al'bomnaia fabrika A. Petersena v S.-Peterburgie." Vladimir Aleksandrovich, Grand Duke of Russia (1847-1909) - former owner. Slavic Recon Project. Metropolitan New York Library Council, 2008. World's Columbian Exposition (1893: Chicago, Ill.). Russkii otdiel. Exhibitions -Illinois Chicago. Slav. Reserve (Photo) (Vsemirnaia kolumbova vystavka 1893 goda v Chikago) SLV 154 V.A. [Monogram of Grand Duke Vladimir Aleksandrovich, binder's title]; Vsemirnaia Kolumbova vystavka 1893 g. v Chigago. Russkii otdiel; Glavnyi vkhod v Manufakturnyi otdiel; Otdiel Rybovodstva i Rybolovstva; Transportnyi Otdiel. Morskoe Ministerstvo; Otdiel Svobodnykh Iskustv-Upravlenie pocht i Telegrafov; Kozhevennyi Otdiel; Mashinnyi Otdiel; Otdiel Liesovodstva; Obshchii vid Manufakturnago Otdiela; Otdiel Svobodnykh Iskustv. Voennoe Ministerstvo; Otdiel Sel'skago Khoziaistva; Transportnyi Otdel; Otdiel uchrezhdennago s Vsemilostivieishago Eia Imperatorskago Velichestva soizvoleniia Damskago Komiteta; Otdiel Vinodieliia; Manufakturnyi Otdiel; Gornyi Otdiel; Manufakturnyi Otdiel; Otdiel Iziashchnykh Iskusstv; Otdiel Iziashchnykh Iskusstv; Otdiel Iziashchnykh Iskusstv; Otdiel KonnozavodstvaLoshadi s zavoda Ego Imperatorskago Vysochestva Velikago Kniazia Dimitriia Konstantinovicha.

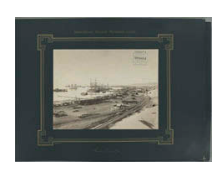

Zakaspiiskaia voennaia zhelieznaia doroga. Ashkhabad: Fotografiia Engelia, [1899?]. 37 mounted photographs, $34 \times 48 \mathrm{~cm}$. "Perl. Jun 18, 1931." Each plate reads: "Zakaspiiskaia voennaia zhelieznaia doroga. Fot. Engelia v Askhabadie." Slavic Recon Project. Metropolitan New York Library Council, 2008. Railroads - Turkmenistan. Zakaspiiskaia zhelieznaia doroga. Slav. Reserve (Photo) (Zakaspiiskaia voennaia zhelieznaia doroga) SLV 155 [Cover title]; Port Uzun-Ada; Gorodok Uzun-Ada; Liesnaia pristan' v UzunAda; Dom nachal'nika distantsiia v Uzun-Ada; Passazhirskaia stantsiia 
v Uzun-Ada; Stantsiia Kodzh; Stantsiia Bami; Fontan na st. Bami; Stantsiia Bakharden; Parovoznoe zdanie na st. Bakharden; Stantsiia Keliata; Stantsiia Geok-Tepe; Kazarma na st. Geok-Tepe; Stantsiia Bezmein; Kazarma na st. Bezmein; Kazarma na 410-i verst; Obshchii vid stantsii Ashkhabad; Parovoi nassos dlia nefti na st. Ashkhabad; Vodokachka na st. Ashkhabad; Vokzal v Askhabade; Stantsiia Artyk; Stantsiia Bada-Durmas; Stantsiia Dzhundzhuklu; Obshchii vid stantsii Dushak; Parovoznoe zdanie na st. Dushak; Passazhirskoe zdanie na st. Dushak; Stantsiia Karrybent; Zhelieznodorozhnyi most cherez r. Tedzhen; Stantsiia Karabata; Stantsiia Merv/obshchii vid/; Zhelieznodorozhnyi most cherez r. Murgab v Mervie; Zhilyia pomieshcheniia dlia agentov dorogi v Mervie; Zhelieznodorozhnaia gostinitsa v Mervie; Stantsiia Bairam-Ali/staryi Merv/; Bassein na st. BairamAli; Amu-Dar'ia u. g. Chardzhuia; Parovye katera "Aleksandr" i "Petr" spushchennye na Amu-Dar'iu 2-ogo Dekabria 1886 g.

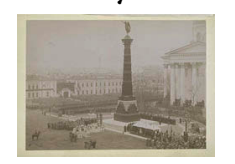

Zhitkov, Georgii Mikhailovich, 1857-1892. Pamiatnik iz turetskikh orudii $v$ S.-Peterburgie, ego sooruzhenie $i$ torzhestvennoe otkrytie 12 oktiabria 1886 g.; vospominanie o turetskoi voinie 1877-1878 godov. St. Petersburg: Tip. i litografiia V.A. Tikhanova, 1889. 84 p.: ill., plans, $19 \times 27 \mathrm{~cm}$. 5 mounted photographs.Alt. title: Pamiatnik iz turetskikh orudii. Four loose leaves of plans inserted. "Perl. Jun 18, 1931." Monuments - Russia. Russo-Turkish War, 1877-1878 - Monuments. Slav. Reserve (Photo) 97-4275 SLV 156 Pamiatnik [left]; Repetitsiia parada 10go-Oktiabria 1886 goda. [right]; [Torzhestvennoe otkrytie pamiatnika]; Vysochaishii obkhod zapasnykh nizhnikh chinov i predstavitelei ot Voisk, sobrannykh na dvore Kazarm L. Gv. 2-oi Artilleriiskoi brigady, gdie dlia nim bylo ustroeno ugoshchenie ot goroda; [Medal'].

\section{Bibliography of Secondary Materials of Photography in Russia}

Andreyev, Leonid. Photographs by a Russian writer, Leonid Andreyev: an undiscoverd portrait of pre-revolutionary Russia. London. [England]: Thames and Hudson, 1989.

Another Russia: through the eyes of the new Soviet photographers. New York, N.Y.: Facts on File Publications, 1986.

Barkhatova, E.V. Russkaia svetopis’. Pervyi vek fotoiskusstva 1839-1914. Sankt-Peterburg: Liki Rossii, 2009.

Brumfield, William Craft. Russkii Sever, svidetel'stvo Uiliama Brumfilda/[interv'iu Nadezhdy Azhgikhinoi]. Moskva: Tri Kvadrata, 2001.

- Sviatyni russkogo Severa: dokumental'no-khudozhestvennye fotografii. Vologda: ARNIKA, 2001.

Chizhova, I. B. (Irina Borisovna). Russkie krasavitsy v proizvedeniiakh zhivopisi i fotografii. Moskva: "Iskusstvo", 1996.

Czapliński, Czesław. Jerzy Kosiński 1933-1991/[fotografie Czesława Czaplińskiego].

[New York: New York Public Library, 2002]

Fotograf Aleksei Mazurin: russkaia fotografiia, 1890-1910-e. Moskva: Punktum, 2005. 
Fotografii na pamiat': fotografy Nevskogo prospekta, 1850- Sankt-Peterburg: Slaviia, 2003.

Fotografía sovietica: 1940-1991: Sala Parpallo, Palau dels Scala, marzo-abril 1993. [Valencia]: Diputacion Provincial de Valencia, 1993.

Livod jelami $=$ Libiesu dzive $=$ The life of Livs. Riga: Neputns: Lìvõ Kultūr Sidām, 2007

Malinowski, Zygmunt. [John Paul II, Czesław Miłosz: two portraits. [New York: New York Public Library, 2004]

Meisterwerke russischer und deutscher Kunstphotographie um 1900: Sergej Lobovikov und die Brüder Hofmeister. München; New York: Prestel, 1999.

Nevskii prospekt: istoriia Sankt-Peterburga $v$ fotografiiakh, konets XIX-nachalo XX veka [oformlenie I.A. Ozerova]. Moskva: Tsentropoligraf, 2003.

Obolensky, Chloe. The Russian Empire: a portrait in photographs. New York: Random House, 1979.

Penson, Max. Soviet Uzbekistan 1920-1930s. Moskva, 2008

Photography in Russia, 1840-1940/edited by David Elliott. London: Thames and Hudson, 1992.

Rozov, Mark. Mark Rozov: moia istoriia iskusstv = Mark Rosow: meine Geschichte der Künste = Mark Rozov: my history of arts. Moskva: Berezovskii, 1999.

Petr Otsup: prostranstvo revoliutsii: Rossia, 1917-1941 = Piotr Otsup: the space of revolution: Russia, 1917-1941. Moskva: Izd-vo "Golden-Bi”, 2007.

Russia in original photographs, 1860-1920. New York: Scribner, 1977.

Russkaia fotografiia: seredina XIX-nachalo XX veka/sostavitel' Nikolai Nikolaevich Rakhmanov; khudozhnik Aleksandr Fedorovich Bykov; avtor vstupitel'noi stat'i Elena Valentinovna Barkhatova. Moskva: Planeta, 1996.

Shedevry fotografii iz chastnykh sobranii: russkaia fotografiia 1849-1918. Moskva: Punktum, 2003.

Shipova, T. N. Fotografy Moskvy na pamiat' budushchemu, 1839-1930: al'bom-spravochnik. Moskva: Izd-vo obedineniia "Mosgorarkhiv", 2001.

Stigneev, Valeri1. Vek fotografii, 1894-1994: ocherki istorii otechestvennoi fotografii. Moskva: Gos. in-t Iskusstvovedeniia, 2005.

Sviataia prepodobnomuchenitsa Velikaia Kniaginia Elizaveta Feodorovna: foto al'bom. Moskva: Novospasskii monastyr', 2007.

Tolstaia, S. A. (Sofia Andreevna). Song without words: the photographs \& diaries of Countess Sophia Tolstoy. Washington, D.C.: National Geographic, 2007.

Villie, Mikhail IAkovlevich. XIX vek v fotografiiakh: iz kollektsii Mikhaila Villie. Moskva, 2006.

Waters, Erin. Cataloguing empire: photographic typologies as a record of late imperial Russia (1839-1917). Thesis (B.A.) Hamilton College, 2001. 